STRUCTURAL

CHEMISTRY

ISSN 2053-2296

Received 3 September 2020

Accepted 7 October 2020

Edited by A. G. Oliver, University of Notre Dame, USA

Keywords: bedaquiline; bedaqulinium salt; drug-resistant tuberculosis; crystal structure.

CCDC references: 2036005; 2036004; 2036003; 2036002; 2036001

Supporting information: this article has supporting information at journals.iucr.org/c

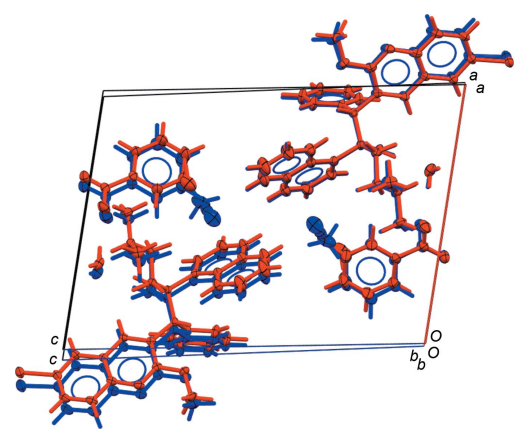

OPEN $\odot$ ACCESS

\section{Crystal structures of salts of bedaquiline}

\author{
Mercy Okezue, ${ }^{a}$ Daniel Smith, ${ }^{a}$ Matthias Zeller, ${ }^{b} *$ Stephen R. Byrn, ${ }^{\mathrm{a}, \mathrm{c}}$ Pamela \\ Smith, ${ }^{d}$ Susan Bogandowich-Knipp, ${ }^{\text {e Dale K. Purcell }}$ and Kari L. Clase ${ }^{\mathrm{a}, \mathrm{g}}$
}

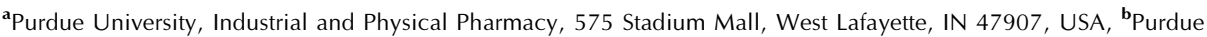
University, Chemistry, 560 Oval Drive, West Lafayette, IN 47907-2084, USA, 'Improved Pharma, LLC, 1281 Win Hentschel Boulevard, West Lafayette, IN 47906, USA, dLeading with Smart Science, LLC, 5315 Shootingstar Lane, West Lafayette, IN 47906, USA, ${ }^{\mathbf{e}}$ Ravine Pharmaceuticals, LLC, 3425 DuBois Street, West Lafayette, IN 47906, USA, ${ }^{\mathbf{f}}$ Chemical Microscopy, LLC, 1281 Win Hentschel Boulevard, West Lafayette, IN 47906, USA, and ${ }^{\mathbf{g}}$ Department of Agricultural \& Biological Engineering, Biotechnology Innovation and Regulatory Science Center, Lilly Hall of Life Sciences, Purdue University, 915 State Street, West Lafayette, IN 47906, USA. *Correspondence e-mail: zeller4@purdue.edu
\end{abstract}

Bedaquiline [systematic name: 1-(6-bromo-2-methoxyquinolin-3-yl)-4-(dimethylamino)-2-(naphthalen-1-yl)-1-phenylbutan-2-ol, $\left.\mathrm{C}_{32} \mathrm{H}_{31} \mathrm{BrN}_{2} \mathrm{O}_{2}\right]$ is one of two important new drugs for the treatment of drug-resistant tuberculosis (TB). It is marketed in the US as its fumarate salt \{systematic name: [4-(6bromo-2-methoxyquinolin-3-yl)-3-hydroxy-3-(naphthalen-1-yl)-4-phenylbutyl]dimethylazanium 3-carboxyprop-2-enoate, $\mathrm{C}_{32} \mathrm{H}_{32} \mathrm{BrN}_{2} \mathrm{O}_{2}{ }^{+} \cdot \mathrm{C}_{4} \mathrm{H}_{3} \mathrm{O}_{4}{ }^{-}$, , and about a dozen other salts of bedaquiline have been described in patent literature, but none have so far been structurally described. In a first communication, we present the crystal structure of bedaquilinium fumarate and of two new benzoate salts, as well as that of a degradation product of the reaction of bedaquilinium fumarate with sodium ethoxide, 3-benzyl-6-bromo-2methoxyquinoline, $\mathrm{C}_{17} \mathrm{H}_{14} \mathrm{BrNO}$. The fumarate and benzoate salts both feature cations monoprotonated at the dimethylamino group. The much less basic quinoline $\mathrm{N}$ atom remains unprotonated. Both salts feature a 1:1 cation-to-anion ratio, with the fumarate being present as monoanionic hydrofumarate. The conformations of the cations are compared to that of free base bedaquiline and with each other. The flexible backbone of the bedaquiline structure leads to a landscape of conformations with little commonalities between the bedaquiline entities in the various structures. The conformations are distinctively different for the two independent molecules of the free base, the two independent molecules of the hydrofumarate salt, and the one unique cation of the benzoate salt. Packing of the salts is dominated by hydrogen bonding. Hydrogen-bonding motifs, as well as the larger hydrogen-bonded entities within the salts, are quite similar for the salts, despite the vastly differing conformations of the cations, and both the hydrofumarate and the benzoate structure feature chains of hydrogenbonded anions that are surrounded by and hydrogen bonded to the larger bedaquilinium cations, leading to infinite broad ribbons of anions, cations, and (for the benzoate salt) water molecules. The benzoate salt was isolated in two forms: as a 1.17-hydrate $\left(\mathrm{C}_{32} \mathrm{H}_{32} \mathrm{BrN}_{2} \mathrm{O}_{2}{ }^{+} \cdot \mathrm{C}_{7} \mathrm{H}_{5} \mathrm{O}_{2}{ }^{-} \cdot 1.166 \mathrm{H}_{2} \mathrm{O}\right)$, obtained from acetone or propanol solution, with one fully occupied water molecule tightly integrated into the hydrogen-bonding network of anions and cations, and one partially occupied water molecule [refined occupancy 16.6 (7)\%], only loosely hydrogen bonded to the quinoline $\mathrm{N}$ atom. The second form is an acetonitrile solvate $\left(\mathrm{C}_{32} \mathrm{H}_{32} \mathrm{BrN}_{2} \mathrm{O}_{2}{ }^{+} \cdot \mathrm{C}_{7} \mathrm{H}_{5} \mathrm{O}_{2}{ }^{-} \cdot 0.742 \mathrm{CH}_{3} \mathrm{CN} \cdot \mathrm{H}_{2} \mathrm{O}\right)$, in which the partially occupied water molecule is replaced by a $74.2(7) \%$-occupied acetonitrile molecule. The partial occupancy induces disorder for the benzoate phenyl ring. The acetonitrile solvate is unstable in atmosphere and converts into a form not distinguishable by powder XRD from the 1.17-hydrate.

\section{Introduction}

Bedaquiline, 1, is one of two important new drugs for the treatment of drug-resistant tuberculosis (TB). Bedaquiline is 
marketed in the US as the fumarate salt (2) with the trade name Sirturo (Brigden et al., 2015). The fumarate salt is described in US Patent 8546428 (Hegyi et al., 2013). The citrate, sulfate, phosphate, and tartrate salts are described in two other patents (Zvatora, Dammer, Krejcik et al., 2016; Zvatora, Dammer, Ridvan et al., 2016). However, none of these salts has been structurally described in detail. For the fumarate, as well as one each of the two sulfate and citrate salts, well-resolved powder X-ray patterns have been reported, but the structures were not solved and no singlecrystal data are reported. For the remaining salts (the phosphate and tartrate salts, and the second sulfate and citrate polymorphs), the powder patterns indicate the samples to have either extremely small particle distributions or to be entirely amorphous. Detailed structural data are reported solely for the free base form of bedaquiline (Petit et al., 2007).
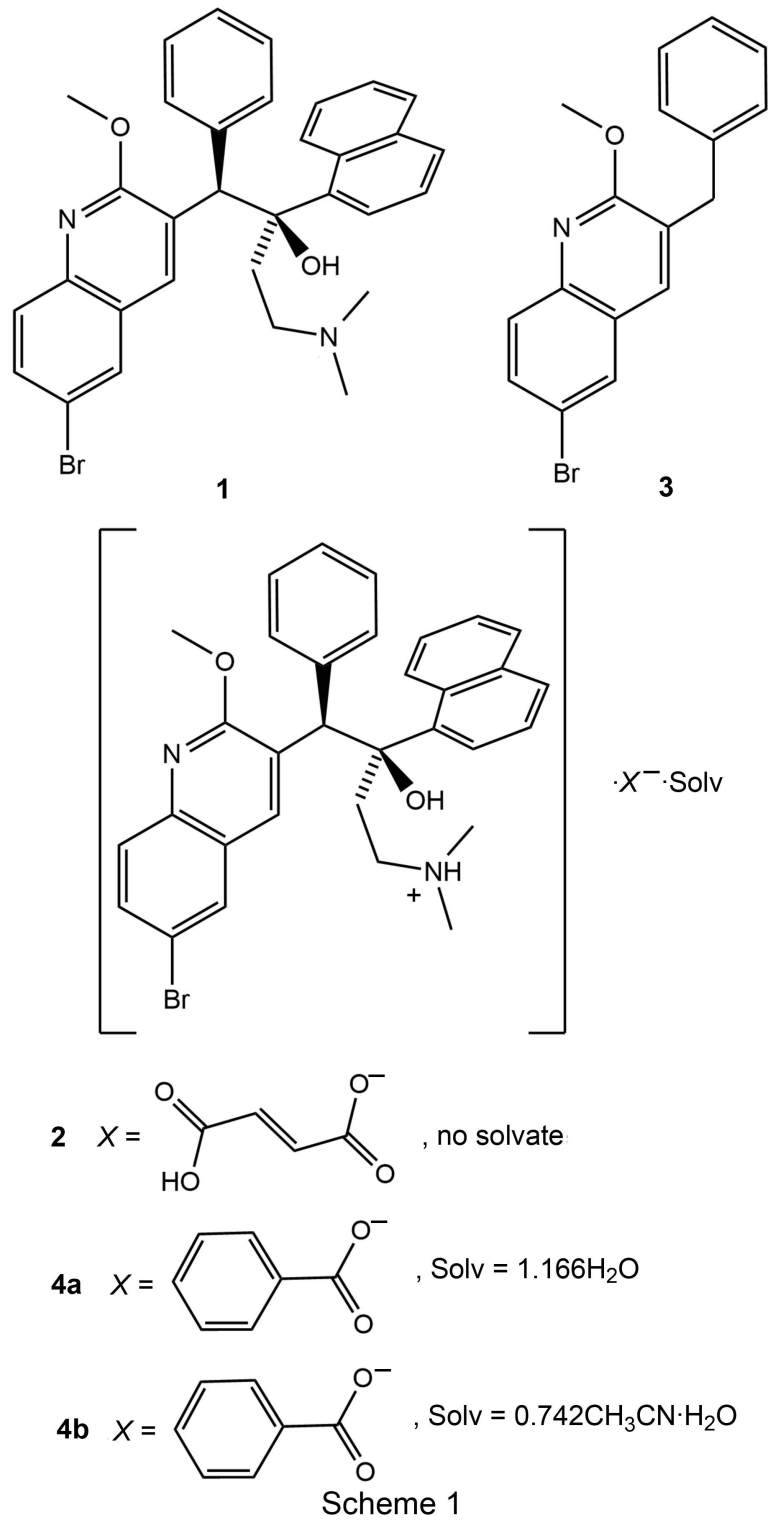

Bedaquiline features two basic $\mathrm{N}$ atoms that are amenable to protonation, i.e. the tertiary amine appended to the dangling ethylene group and the pyridine $\mathrm{N}$ atom. The two sites have distinctly different basicities and selective protonation of only the more basic amine site should be possible. Salts of both mono- and dicationic bedaquilinium ions can thus be formed, depending on the strength and amount of acid used for salt formation. The formation of cocrystals (with no or incomplete proton transfer) can also be imagined.

The NMR data reported in the patent publications indicate bedaquilinium fumarate to have a 1:1 anion-to-cation ratio. Whether the bedaquiline is protonated once or twice (and the fumarate deprotonated once or twice) had not been disclosed. For the sulfate salts, a 1:1 molar ratio of bedaquiline-tosulfuric acid was used, but the anion-to-cation ratio in the salt was not determined. The given reaction yields, assuming a 1:1 salt, are around $33 \%$, which would allow formation of a 1:2, a 1:1, or a $2: 1$ salt. The patent specifically states a wide range for the molar ratio of bedaquiline to sulfuric acid: 'The molar ratio of bedaquiline:sulfuric acid may be in the range of 10:1 to 1:3, preferably $1: 1,2: 1$, and 1:2'. Similar statements have been made for the tartrate salts, one of the citrate salts, and the phosphate salt. No elemental analysis data are given to support any of the possible ratios, thus leaving the stoichiometry and the overall nature of the presented salts in question. The possibility of hydrate or solvate formation was also not properly addressed in the patent claims.

This lack of structural knowledge and even of basic chemical composition frustrates the understanding of the chemical, physical, and physiological properties of bedaquiline and its derivatives. To reduce this paucity of information on the bedaquiline system, there is interest in developing additional salts of bedaquiline and obtaining detailed analysis and structural data for these compounds, to better understand and possibly improve their properties, such as solubility, which in turn affect pharmacokinetics and dosage. Additional reasons for this study include finding a bedaquiline salt with improved stability and hygroscopicity.

\section{Experimental}

Melting points were determined using a Thomas Hoover Capillary Melting Point apparatus and are uncorrected. NMR data were collected in acetonitrile- $d_{3}\left(\mathrm{ACN}-d_{3}\right)$ using a Bruker DRX-500 spectrometer and were referenced against the residual nondeuterated solvent peak.

Benzoic acid was purchased from Mallinckrodt, acetone from Fischer Chemicals, and acetonitrile from VWR Chemicals. Hydrochloride in methanol $1.25 M$ was obtained from Fluka. Bedaquiline fumarate was obtained from Johnson \& Johnson. All chemicals were used as received without further purification.

Polarized light microscopy images were collected using an Olympus Series BX51TRF (Olympus America Inc., Melville, $\mathrm{NY}$ ) polarized light microscope equipped with $12 \mathrm{~V} / 100 \mathrm{~W}$ illumination; an Achromat 0.9 NA polarized-light condenser; Olympus Series UPlanFL N objectives: 40X/0.75 NA, 20X/ $0.50 \mathrm{NA}, 10 \mathrm{X} / 0.30 \mathrm{NA}$, and 4X/0.13 NA; an intermediate tube with variable position analyzer and compensator; and a trinocular viewing head with a Lumenera Series Infinity X 
(Teledyne Lumenera, Ottawa, Ontario, Canada) digital camera using Infinity (Version 6.5.6) and Infinity Analyze software (Version 7.0.2.930, Build date 01-Feb-2020). A small portion of sample was placed on a cleaned microscope slide and a No. 1 1/2-cover glass placed over the sample. Mineral oil, USP (CAS: 8042-47-5), was allowed to cover the sample by capillarity. Images were acquired as a collection of three: (i) plane polarized light, (ii) crossed polarized light, and (iii) crossed polarized light with a first-order red compensator. Microscopy observations revealed crystal habits for bedaquilinium benzoate powders as birefringent platy anhedral agglomerates that are softly bound and easily dispersed under light pressure from a tungsten needle on the cover glass. A representative collection of images is given in the supporting information.

IR microspectroscopy experiments were conducted using a Smiths Detection (Danbury, CT) IlluminatIR 1.5 IR Microspectrometer accessory on an Olympus Series BX41TF polarized-light microscope (Olympus America Inc., Melville, NY), which provided the base optical platform. The IlluminatIR 1.5 is equipped with a gray-body ceramic IR source, a $60^{\circ}$ Michelson Interferometer with a zinc-selenide $(\mathrm{ZnSe})$ beam splitter, a 4 wavenumber $\left(\mathrm{cm}^{-1}\right)$ spectral resolution, and a $0.25 \times 0.25 \mathrm{~mm}$ liquid-nitrogen-cooled mercury cadmium telluride (MCT) photoconductive detector, and the sample area was defined using a fixed circular $100 \mu \mathrm{m}$ aperture. The IlluminatIR 1.5 is computer-interfaced using universal serial bus (USB) communications with Smiths Detection QualID App (Version 2.51, 2005) software. Advanced data processing was conducted using either Thermo Galactic spectral analysis software packages GRAMS/AI and SpectralID, or Thermo Fisher Scientific OMNIC software (Version 9.11.706, 2020). IR analyses were performed by reflection/absorption (R/A) using an all-reflecting objective (ARO, 15X, $0.88 \mathrm{NA}$ ). A small amount of sample was transferred to a low-E microscope slide (Smiths Detection P/N: 006-4013) and dispersed to a thin layer. IR microprobe analyses were conducted on what appeared microscopically to be a single crystal. An FT-IR spectral background was collected immediately prior to each sample spectral analysis.

Powder X-ray diffraction (XRD) data were collected in focusing mode on a PANalytical Empyrean X-ray diffractometer equipped with Bragg-Brentano HD optics, a sealedtube copper X-ray source $(\lambda=1.54178 \AA)$, Soller slits on both the incident and receiving optics sides, and a PixCel3D Medipix detector. Samples were hand ground using an agate mortar and pestle, and packed into a silicon single-crystal zero-background sample holder, $16 \mathrm{~mm}$ wide and $0.25 \mathrm{~mm}$ deep. Antiscatter slits and divergence slits, as well as the mask, were chosen based on sample area and starting $\theta$ angle. Data were collected between 4 and $40^{\circ}$ in $2 \theta$ under ambient conditions using the PANalytical Data Collector software (PANalytical, 2015). Rietveld refinements were performed against the $150 \mathrm{~K}$ models of the single-crystal structure data sets using HighScore (PANalytical, 2015) software. Refinement of preferred orientation was included using a spherical harmonics model. Plots of Rietveld fits for all compounds are given in the supporting information.

\subsection{Synthesis and crystallization}

2.1.1. Free base bedaquiline (1). The free base used during synthesis was prepared by extracting a $\mathrm{CH}_{2} \mathrm{Cl}_{2}$ solution of the fumarate three times with saturated $\mathrm{NaHCO}_{3}$ solution (Rombouts et al., 2016). The identity and purity of the free base thus afforded from the material supplied by Johnson \& Johnson was verified using NMR spectroscopy [m.p. 175$176{ }^{\circ} \mathrm{C}$; literature value $181^{\circ} \mathrm{C}$ (Zvatora, Dammer, Ridvan et $a l ., 2016)] .{ }^{1} \mathrm{H}$ NMR $\left(500 \mathrm{MHz}, \mathrm{ACN}-d_{3}\right): \delta 8.82(s, 1 \mathrm{H}), 8.66$ $(d, J=8.7 \mathrm{~Hz}, 1 \mathrm{H}), 8.03(s, 1 \mathrm{H}), 8.02(d, J=7.3 \mathrm{~Hz}, 1 \mathrm{H}), 7.87$ $(d, J=8.0 \mathrm{~Hz}, 1 \mathrm{H}), 7.66(t, J=7.7 \mathrm{~Hz}, 4 \mathrm{H}), 7.49(t, J=7.7 \mathrm{~Hz}$, $1 \mathrm{H}), 7.30(\mathrm{~m}, 3 \mathrm{H}), 6.87(\mathrm{~m}, 3 \mathrm{H}), 5.88(\mathrm{~s}, 1 \mathrm{H}), 4.20(\mathrm{~s}, 3 \mathrm{H}), 2.52$ $(d, J=14.6 \mathrm{~Hz}, 1 \mathrm{H}), 2.01(m, 2 \mathrm{H}), 1.89(m, 7 \mathrm{H})$.

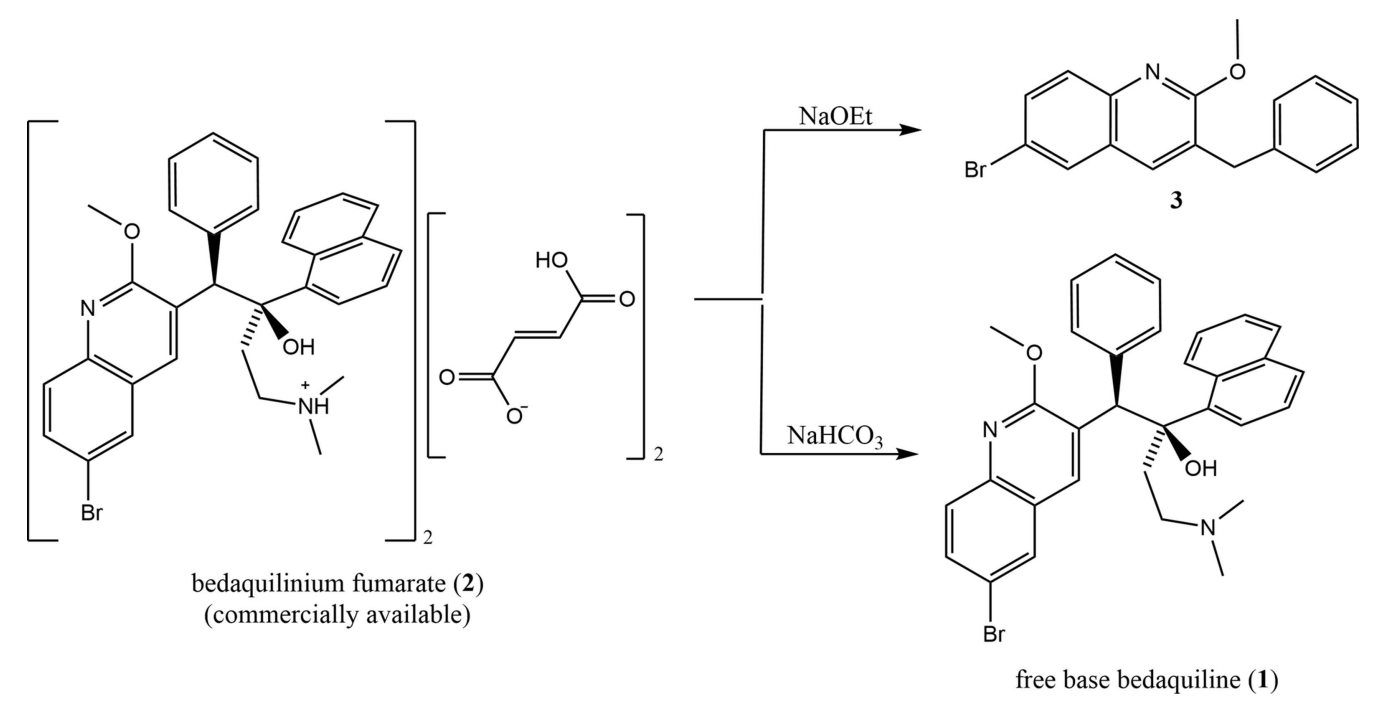

Figure 1

Isolation of free base bedaquiline (1) from commercially available bedaquilinium fumarate (2), and decomposition to $\mathbf{3}$. 
Table 1

Experimental details.

Experiments were carried out at $150 \mathrm{~K}$. Absorption was corrected for by multi-scan methods (SADABS2016; Krause et al., 2015).

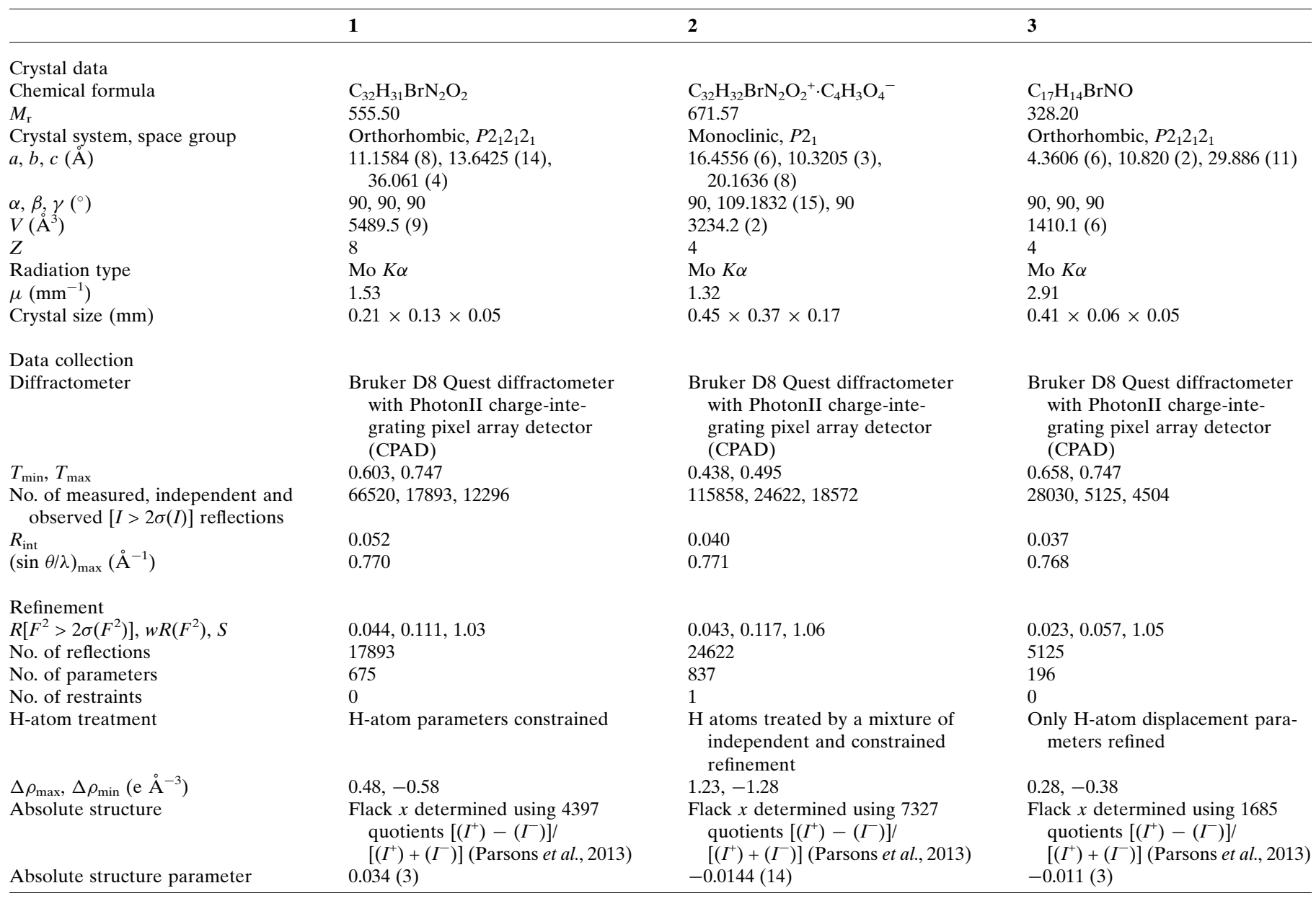

$4 \mathbf{a}$

Crystal data

Chemical formula

$M_{\mathrm{r}}$

Crystal system, space group

$a, b, c(\AA)$

$\alpha, \beta, \gamma\left({ }^{\circ}\right)$

$V\left(\AA^{3}\right)$

$Z$

Radiation type

$\mu\left(\mathrm{mm}^{-1}\right)$

Crystal size (mm)

Data collection

Diffractometer

$T_{\min }, T_{\max }$

No. of measured, independent and observed $[I>2 \sigma(I)]$ reflections

$R_{\text {int }}$

$(\sin \theta / \lambda)_{\max }\left(\AA^{-1}\right)$

$\mathrm{C}_{32} \mathrm{H}_{32} \mathrm{BrN}_{2} \mathrm{O}_{2}{ }^{+} \cdot \mathrm{C}_{7} \mathrm{H}_{5} \mathrm{O}_{2}{ }^{-} \cdot 1.166 \mathrm{H}_{2} \mathrm{O}$

698.70

Monoclinic, $P 2_{1}$

12.6384 (5), 7.9259 (3), 17.5249 (8)

90, 99.8450 (17), 90

$1729.63(12)$

2

Mo $K \alpha$

1.24

$0.55 \times 0.21 \times 0.13$

Bruker D8 Quest diffractometer with PhotonII charge-integrating pixel array detector

(CPAD)

$0.638,0.746$

80228, 13080, 10456

0.049

0.770

$0.032,0.073,1.03$

13080

445

5

$\mathrm{H}$ atoms treated by a mixture of independent and constrained refinement

$4 b$

$\mathrm{C}_{32} \mathrm{H}_{32} \mathrm{BrN}_{2} \mathrm{O}_{2}{ }^{+} \cdot \mathrm{C}_{7} \mathrm{H}_{5} \mathrm{O}_{2}{ }^{-} \cdot 0.742 \mathrm{C}_{2} \mathrm{H}_{3} \mathrm{~N} \cdot \mathrm{H}_{2} \mathrm{O}$ 726.10

Monoclinic, $P 2_{1}$

12.8661 (8), 8.0386 (5), 17.4704 (10)

$90,101.093(3), 90$

$1773.13(19)$

2

$\mathrm{Cu} K \alpha$

1.97

$0.31 \times 0.05 \times 0.05$

Bruker D8 Quest diffractometer with PhotonIII_C14 charge-integrating and photon counting pixel array detector $0.599,0.754$

$39739,7360,6750$

0.060

0.639

$0.035,0.085,1.06$

7360

515

195

$\mathrm{H}$ atoms treated by a mixture of independent and constrained refinement 
Table 1 (continued)

\begin{tabular}{lll}
\hline & $\mathbf{4 a}$ & $\mathbf{4 b}$ \\
\hline$\Delta \rho_{\max }, \Delta \rho_{\min }\left({\left.\mathrm{e} \AA^{-3}\right)}^{-3}\right.$ & $0.36,-0.48$ & $0.40,-0.50$ \\
Absolute structure & Flack $x$ determined using 4051 quotients & $\begin{array}{c}\text { Flack } x \text { determined using } 2778 \text { quotients } \\
{\left[\left(I^{+}\right)-\left(I^{-}\right)\right] /\left[\left(I^{+}\right)+\left(I^{-}\right)\right](\text {Parsons } \text { et al., 2013) }}\end{array}$ \\
Absolute structure parameter & $0.006(3)$ & $0.004(8)$
\end{tabular}

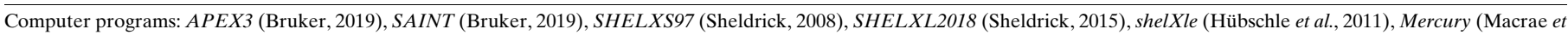
al., 2020) and publCIF (Westrip, 2010).

Single crystals were grown by dissolving bedaquiline ( $30 \mathrm{mg}, 0.054 \mathrm{mmol})$ in acetone $(1 \mathrm{ml})$ in a $5 \mathrm{ml}$ scintillation vial and the solution was allowed to evaporate slowly to obtain medium-sized plate-shaped crystals of $\mathbf{1}$.

2.1.2. Decomposition of bedaquiline fumarate by sodium ethoxide. Sodium ethoxide ( $1.5 \mathrm{~g}, 22.0 \mathrm{mmol}$ ) was dissolved in EtOH $(20 \mathrm{ml})$. The resulting solution was added to a solution of bedaquiline fumarate $(5 \mathrm{~g}, 7.44 \mathrm{mmol})$ in $\mathrm{ACN} / \mathrm{EtOH}$ $(50 \mathrm{ml}, 1: 1 \mathrm{v} / \mathrm{v})$. After $1 \mathrm{~h}$, water was added slowly and the resulting mixture extracted with EtOAc. The combined organic layers were dried $\left(\mathrm{MgSO}_{4}\right)$ and then concentrated to provide a colorless crystalline material that was found by IR and NMR spectroscopies to not match free base bedaquiline. Individual crystals were identified as 3-benzyl-6-bromo-2methoxyquinoline (3) by single-crystal X-ray diffraction, and no further analyses were performed.

2.1.3. Bedaquilinium fumarate (2). Bedaquiline (30 mg, $0.054 \mathrm{mmol})$ was mixed with fumaric acid $(6.3 \mathrm{mg}$, $0.054 \mathrm{mmol})$ dissolved in acetone $(1 \mathrm{ml})$ in a $10 \mathrm{ml}$ scintillation vial. Propyl alcohol $(5 \mathrm{ml})$ was then added and the mixture was allowed to evaporate slowly to obtain large colorless blockshaped crystals that were analyzed by single-crystal and powder X-ray diffraction. ${ }^{1} \mathrm{H}$ NMR $\left(500 \mathrm{MHz}, \mathrm{ACN}-d_{3}\right): \delta$ $8.68(d, J=8.3 \mathrm{~Hz}, 1 \mathrm{H}), 8.55(s, 1 \mathrm{H}), 8.05(d, J=7.2 \mathrm{~Hz}, 1 \mathrm{H})$, $7.97(s, 1 \mathrm{H}), 7.88(d, J=7.9 \mathrm{~Hz}, 1 \mathrm{H}), 7.66(m, 4 \mathrm{H}), 7.51(t, J=$ $7.2 \mathrm{~Hz}, 1 \mathrm{H}), 7.32(m, 3 \mathrm{H}), 6.89(m, 3 \mathrm{H}), 6.32(s, 2 \mathrm{H}), 5.89(s$, $1 \mathrm{H}), 4.21(\mathrm{~s}, 3 \mathrm{H}), 3.02(\mathrm{~m}, 1 \mathrm{H}), 2.69(\mathrm{~m}, 1 \mathrm{H}), 2.24(\mathrm{~s}, 7 \mathrm{H}), 2.09$ $(m, 2 \mathrm{H})$.

2.1.4. Bedaquilinium benzoates $4 \mathrm{a}$ and $4 \mathrm{~b}$. Bedaquiline (30 mg, $0.054 \mathrm{mmol})$ was mixed with benzoic acid $(6.7 \mathrm{mg}$, $0.055 \mathrm{mmol})$. The mixture was dissolved in acetone $(2 \mathrm{ml})$ in a $5 \mathrm{ml}$ scintillation vial and was allowed to evaporate. The 1.17hydrate $4 \mathbf{a}$ was obtained in the form of colorless rod-shaped crystals (m.p. $\left.127-129{ }^{\circ} \mathrm{C}\right) .{ }^{1} \mathrm{H}$ NMR $\left(500 \mathrm{MHz}, \mathrm{ACN}-d_{3}\right): \delta$ $8.75(d, J=8.4 \mathrm{~Hz}, 1 \mathrm{H}), 8.67(s, 1 \mathrm{H}), 8.12(d, J=7.1 \mathrm{~Hz}, 1 \mathrm{H})$, $7.94(d, J=8.0 \mathrm{~Hz}, 1 \mathrm{H}), 7.86(m, 3 \mathrm{H}), 7.75(d, J=8.0 \mathrm{~Hz}, 2 \mathrm{H})$, $7.56(m, 4 \mathrm{H}), 7.38(m, 5 \mathrm{H}), 6.93(m, 3 \mathrm{H}), 5.95(s, 1 \mathrm{H}), 4.26(s$, $3 \mathrm{H}), 3.05(\mathrm{~m}, 1 \mathrm{H}), 2.96(\mathrm{~m}, 1 \mathrm{H}), 2.25(\mathrm{~m}, 7 \mathrm{H}), 1.96(m, 2 \mathrm{H})$. An identical material with the same water content was obtained when crystallization was carried out from 2-propanol instead of acetone.

Bedaquiline $(30 \mathrm{mg}, 0.054 \mathrm{mmol})$ was mixed with benzoic acid $(6.8 \mathrm{mg}, 0.056 \mathrm{mmol})$ in acetonitrile $(10 \mathrm{ml})$ and was allowed to evaporate slowly. The acetonitrile solvate monohydrate $\mathbf{4 b}$ was obtained in the form of thin colorless needles (m.p. $127-129^{\circ} \mathrm{C}$ ).

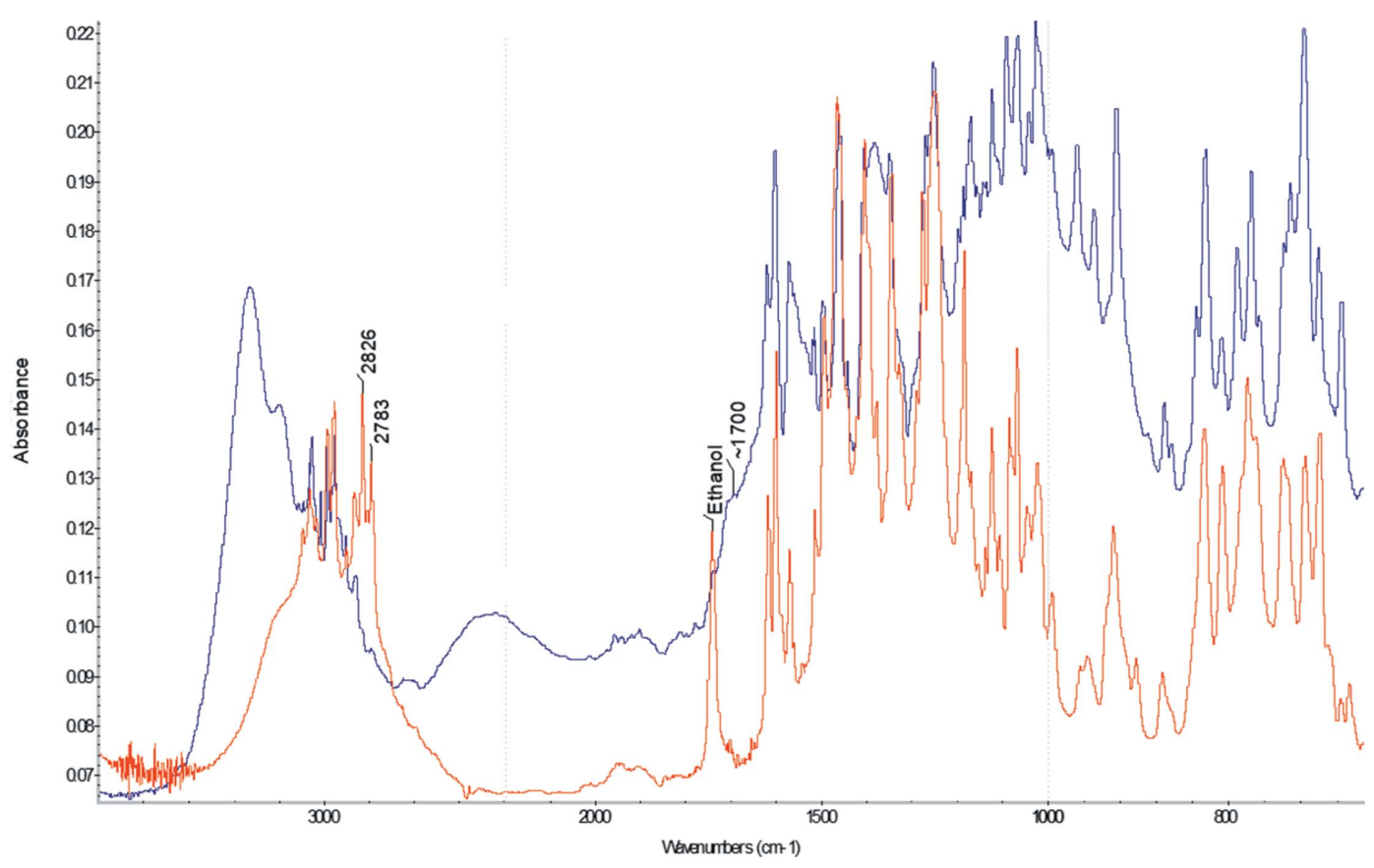

Figure 2

IR spectra of bedaquilinium benzoate (4a) (blue) and bedaquiline free base (1) (red). 


\subsection{Refinement}

Crystal data, data collection, and structure refinement details are summarized in Table 1. The two benzoate salt structures $\mathbf{4 a}$ and $\mathbf{4 b}$ are isomorphous, differing from each other only in the nature of part of the solvent molecules and some slight shifts to other atoms, and they were refined against a common structural model, with the structure of $\mathbf{4 b}$ being solved by isomorphous replacement from that of $\mathbf{4 a}$. The atom-naming scheme for the published bedaquiline free base structure (Petit et al., 2007), as deposited in the Cambridge Structural Database (CSD; Groom et al., 2016; refcode KIDWAW), was used for the remeasured $150 \mathrm{~K}$ data of free base bedaquiline $\mathbf{1}$ and was also adopted for the bedaquilinium cations in the two benzoate salts $\mathbf{4 a}$ and $\mathbf{4 b}$, and fumarate salt 2.

For powder X-ray data collection and refinement, see the Experimental (\$2).

2.2.1. $\mathrm{H}$-atom treatment. $\mathrm{C}$-bound $\mathrm{H}$ atoms were added in calculated positions and refined using a riding model. $\mathrm{C}-\mathrm{H}$ bond distances were constrained to $0.95 \AA$ for aromatic $\mathrm{C}-\mathrm{H}$ moieties, and to 1.00, 0.99, and $0.98 \AA$ for aliphatic $\mathrm{C}-\mathrm{H}, \mathrm{CH}_{2}$, and $\mathrm{CH}_{3}$ moieties, respectively. Alcohol $\mathrm{O}-\mathrm{H}$ and ammonium $\mathrm{N} R_{3} \mathrm{H} \mathrm{N}-\mathrm{H}$ bond distances were either freely refined (for 2 ) or were constrained to 0.84 and $1.00 \AA$, respectively. Methyl $\mathrm{CH}_{3}$ and hydroxy $\mathrm{H}$ atoms were allowed to rotate but not to tip to best fit the experimental electron density. The positions of the carboxylate $\mathrm{H}$ atoms (in 2) were refined freely. The positions of the fully occupied water $\mathrm{H}$ atoms were refined freely and $\mathrm{O}-\mathrm{H}$ distances were restrained to 0.84 (2) $\AA$. The $\mathrm{H}$ atoms of the partially occupied water molecule in $\mathbf{4 a}$ were initially refined and $\mathrm{O}-\mathrm{H}$ and $\mathrm{H} \cdots \mathrm{H}$ distances were restrained to 0.84 (2) and $1.36(2) \AA$, respectively, while a damping factor was applied. The position of water atom H6E (in 4a) was further restrained based on hydrogen-bonding considerations, i.e. to be hydrogen bonded to the pyridine $\mathrm{H}$ atom, with the $\mathrm{H} \cdots \mathrm{N}$ distance restrained to 2.35 (2) $\AA$. In the final refinement cycles, the damping factor was removed and the $\mathrm{H}$ atoms were set to ride on the parent $\mathrm{O}$ atom. For all structures, the $U_{\text {iso }}(\mathrm{H})$ values were set to a multiple of $U_{\text {eq }}(\mathrm{C})$, being 1.5 for $\mathrm{CH}_{3}$ and $\mathrm{OH}$, and 1.2 for $\mathrm{C}-\mathrm{H}, \mathrm{CH}_{2}$, and $\mathrm{N}-\mathrm{H}$ groups, respectively.

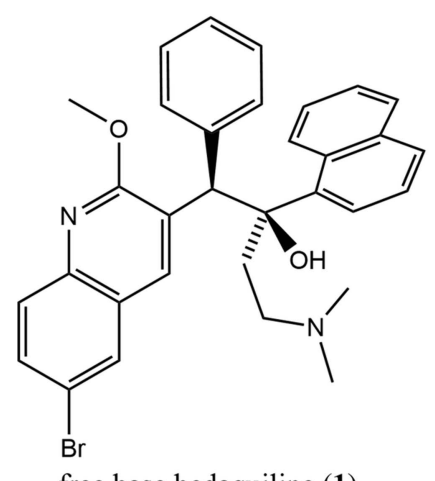

free base bedaquiline (1)

Figure 3

Synthesis of bedaquilinium benzoate (4).

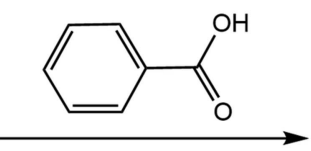

acetone, $25^{\circ} \mathrm{C}$
2.2.2. Disorder modeling. In the structure of $\mathbf{4 a}$, one fully occupied and one partially occupied water molecule are present in the lattice. The occupancy ratio refined to 0.166 (7). In $\mathbf{4 b}$, the partially occupied water molecule is replaced by an approximately three-quarter-occupied acetonitrile molecule. In the absence of the acetonitrile molecule, the neighboring benzene ring of the benzoate anion tilts slightly to move towards the void left by the absent solvent molecule. The two disordered benzene moieties were restrained to have similar geometries. The $U^{i j}$ components of the anisotropic displacement parameters (ADPs) for disordered atoms closer to each other than $2.0 \AA$ were restrained to be similar. The ADPs of the ipso $\mathrm{C}$ atoms, which occupy nearly identical positions, were constrained to be identical. Subject to these conditions, the occupancy ratio refined to 0.742 (7):0.258 (7) in favor of the acetonitrile molecule being present.

\section{Salt screening and methods}

Salt screening is a complex and challenging endeavor involving potentially millions of experiments. For bases like bedaquiline, these experiments can involve up to 50 commonly used salt formers in various ratios, as well as crystallizations from up to 60 different solvents by varying temperature, concentration, agitation, $\mathrm{pH}$, and other factors. Further mixtures of these solvents can be used. Antisolvent crystallization using these solvents is also of interest and introduces even more variables.

For bedaquiline, the first step in screening for additional salts involved recovering bedaquiline free base from the commercially available bedaquilinium fumarate. This was first attempted by deprotonation of the bedaquilinium cation of the fumarate salt using the base sodium ethoxide. However, the alkoxide, when used in excess, proved to be too strong a base and led to fragmentation of the bedaquiline molecule. One of the products of the decomposition reaction was isolated by crystallization and identified, by single-crystal X-ray diffraction, as 3-benzyl-6-bromo-2-methoxyquinoline (3) (Fig. 1) and its structure will be described below. The other half of the decomposition reaction was not recovered or identified, but is assumed to be the ketone elimination product

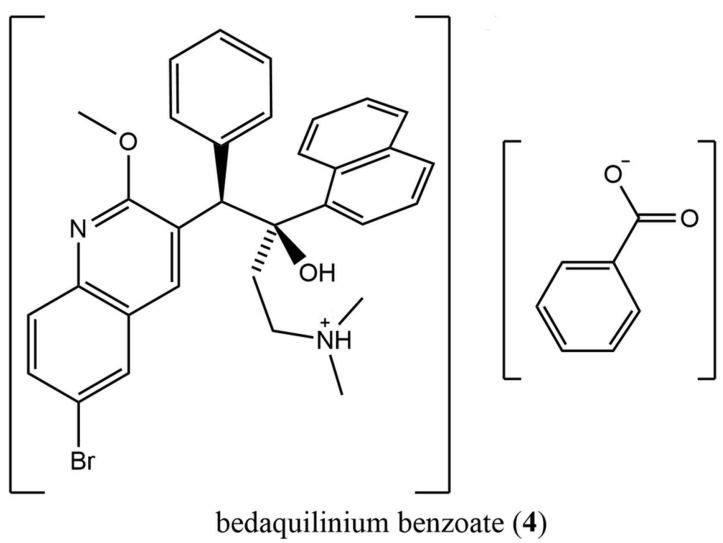

bedaquilinium benzoate (4) 
of the remaining bedaquiline fragment, 4-(dimethylamino)-1(naphthalen-1-yl)butan-2-one. The reaction most likely proceeds through initial deprotonation of all acidic groups by the ethoxide, including the central alcohol of bedaquiline. The tertiary alkoxide thus formed can undergo a reverse Grignard reaction (Zook et al., 1959), under elimination of the ketone and the carbanion of $\mathbf{3}$. Using much less basic sodium bicarbonate as the neutralizing agent avoids this decomposition reaction. Bedaquiline free base could be recovered that way from the fumarate salt, following the procedure described by Rombouts et al. (2016), thus allowing us to proceed to use the free base in salt screening experiments (Fig. 1).

Because the fumarate, citrate, sulfate, phosphate, and tartrate salts were known, salt formation screening focused on the crystallization of salts such as acetate, benzoate, benzenesulfonate, hydrobromide, succinate (1:1 and 1:2), hydrochloride, tartrate (1:1 and 1:2), lactate, maleate (1:1 and 1:2), malate (1:1 and 1:2), and mesylate. In general, the crystallizations involved mixing stoichiometric amounts of bedaquiline with the acids at either 1:1 or 1:2 molar ratios in acetone, acetonitrile, tetrahydrofuran, and ethyl acetate, either with or without the antisolvents water and hexane. The solvents were evaporated either slowly or rapidly, and materials were typically first screened using polarized-light microscopy (PLM) to ensure that a crystalline material had formed, and that the sample was uniform. Samples that passed the first screening step were submitted for further analysis. Crystals were analyzed by NMR (dissolved in an appropriate solvent) to confirm the presence of both components in the material. In the next step, materials were further screened using IR microspectroscopy, to confirm that the material was indeed a new substance (a salt or a cocrystal), and not just a mixture of the two components. Although some investigators have advanced the theory that Raman spectroscopy is the best method for analysis and determination of salt formation (e.g. Kojima et al., 2010), we found IR microspectroscopy had better specificity than Raman microscopy for the bedaquiline free base and salts; therefore, screening materials via Raman methods was abandoned. IR microspectroscopy proved to be a superior method to determine the formation of bedaquilinium salts. Materials that passed these screening steps (PLM, NMR, and IR spectroscopy) were then analyzed by powder $\mathrm{X}$-ray diffraction. Rietveld refinement was used to identify known crystal phases. For samples for which suitable crystals could be obtained, single-crystal X-ray diffraction was used to determine the structures of phases not yet structurally described.

Example IR spectra comparing bedaquilinium benzoate and free base bedaquiline are given in Fig. 2; see Fig. 3 for the synthesis. The spectra are distinctly different, indicating transformation of the free base into a material containing both benzoate and bedaquiline fragments. A range of bands in the fingerprint region indicate the presence of a bedaquiline component in both compounds. A shoulder near $1700 \mathrm{~cm}^{-1}$ in the bedaquiline benzoate spectrum can be assigned to the $\mathrm{C}=\mathrm{O}$ stretch of benzoate, confirming the formation of the salt. Further evidence for the formation of a salt, rather than a simple mixture of the two starting materials, is provided by the absence of bands in the range $2830-2760 \mathrm{~cm}^{-1}$. Tertiary amines (of which the free base is one) have a characteristic $\mathrm{N}-\mathrm{CH}_{2}$ in-phase stretch that occurs in this range (Colthup et al., 1990). The bands in this range of the free base spectrum are not present in the bedaquiline benzoate spectrum, suggesting the formation of a salt. Note: the free base spectrum contains some spectral features due to ethanol, which was used in the synthesis process.

In the course of our investigations, we had been so far able to determine the single-crystal structures of bedaquilinium fumarate (2), the commercially available form of bedaquiline, as well as isolate and characterize two other previously unknown bedaquilinium salts: the mono-benzoate salt, in the form of its 1.17-hydrate (4a), and a mixed hydrate acetonitrile solvate (4b). Their structures, as well as that of the degradation product from reaction of bedaquilinium fumarate with sodium ethoxide, 3-benzyl-6-bromo-2-methoxyquinoline (3), will be described below. The structure of free base bedaquiline (1) was re-recorded at $150 \mathrm{~K}$ for easier comparison with the low-temperature data of $\mathbf{2}, \mathbf{4 a}$, and $\mathbf{4 b}$. Implications for the larger bedaquiline system will be discussed.

\section{Results and discussion}

3-Benzyl-6-bromo-2-methoxyquinoline (3), the solvolysis product from reaction of bedaquiline with sodium ethoxide, lacks an easy-to-identify NMR signature and was identified by single-crystal X-ray diffraction. It crystallized from acetonitrile in the orthorhombic and chiral space group $P 2_{1} 2_{1} 2_{1}$ (Fig. 4). The starting bedaquilinium fumarate is a chiral compound and an enantiopure sample was used. This chiral information and all chiral centers are, however, lost in the degradation reaction to 3-benzyl-6-bromo-2-methoxyquinoline (3). In the solid state, the molecule does, however, exhibit chirality, and the crystal analyzed was found to be enantiopure, with a Flack parameter of -0.011 (3). In solution, the

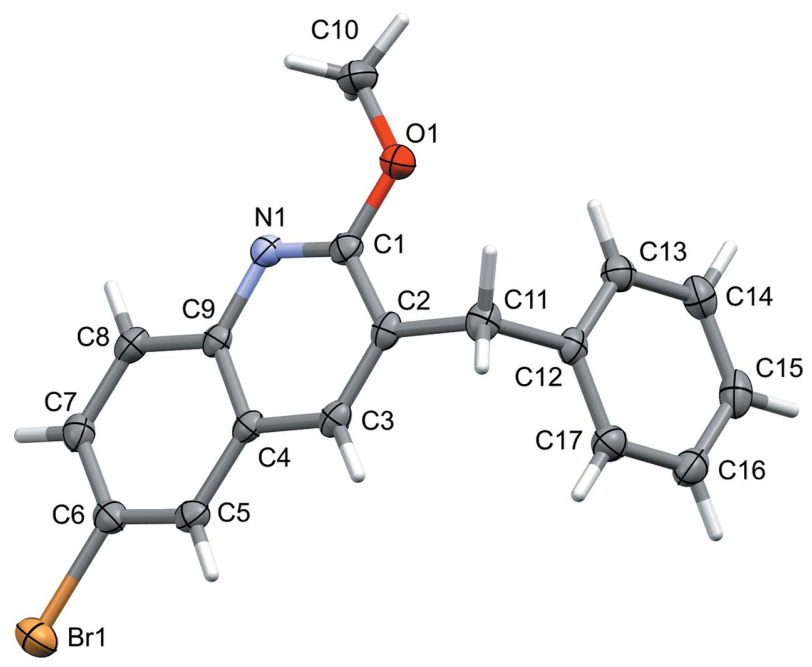

Figure 4

The structure of decomposition product $\mathbf{3}$ (50\% probability displacement ellipsoids). 


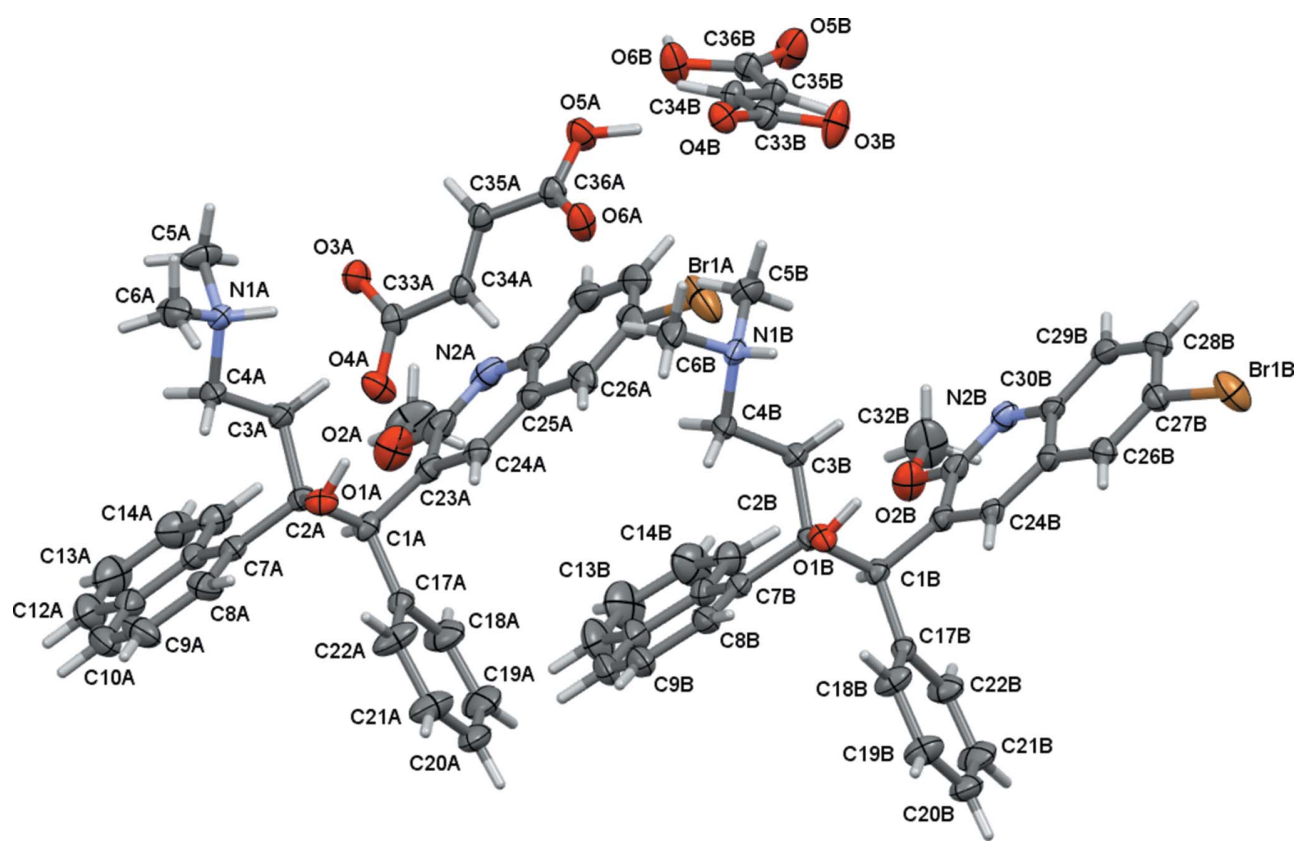

Figure 5

Single-crystal structure of fumarate salt $\mathbf{2}$ (50\% probability displacement ellipsoids).

material is expected to be a rapidly interconverting racemic mixture, as simple rotation of the benzyl group to the other side of the mean plane of the molecule creates the inversionrelated enantiomer. Molecules of $\mathbf{3}$ are divided into two planar fragments: the benzyl group and the 6-bromo-2-methoxyquinoline moiety. Both fragments are close to ideally flat, with r.m.s. deviations from planarity of only 0.0052 and $0.0194 \AA$, respectively. The methoxy group is thus ideally coplanar with the remainder of the bromoquinoline fragment. It points away from the benzyl $\mathrm{CH}_{2}$ group to avoid steric interactions. The torsion angle between the two mean planes is $73.01(4)^{\circ}$.

The structures of the three bedaquilinium salts, i.e. fumarate $\mathbf{2}$, and benzoates $\mathbf{4 a}$ and $\mathbf{4 b}$, are substantially more com- plicated (Figs. 5 and 6), but they share some commonalities. In all three salt structures, the bedaquilinium cation is singly protonated at the dimethylamino fragment, with 1:1 anion-tocation ratios. In the structure of $\mathbf{2}$, the fumarate anions remain singly protonated hydrofumarate $(1-)$ anions, thus being monoanionic, as are the benzoate anions. The quinoline $\mathrm{N}$ atoms remain unprotonated, even though there are additional acidic protons available in the structure of $\mathbf{2}$. At first glance, this might be surprising, since many pyridinium salts of both benzoic and fumaric acids have been reported 973 pyridinium benzoate derivatives and 44 pyridinium fumarate salts are reported in the CSD (Groom et al., 2016), accessed August 2020]. The behaviour is, however, in agreement with the $\mathrm{p} K_{a}$
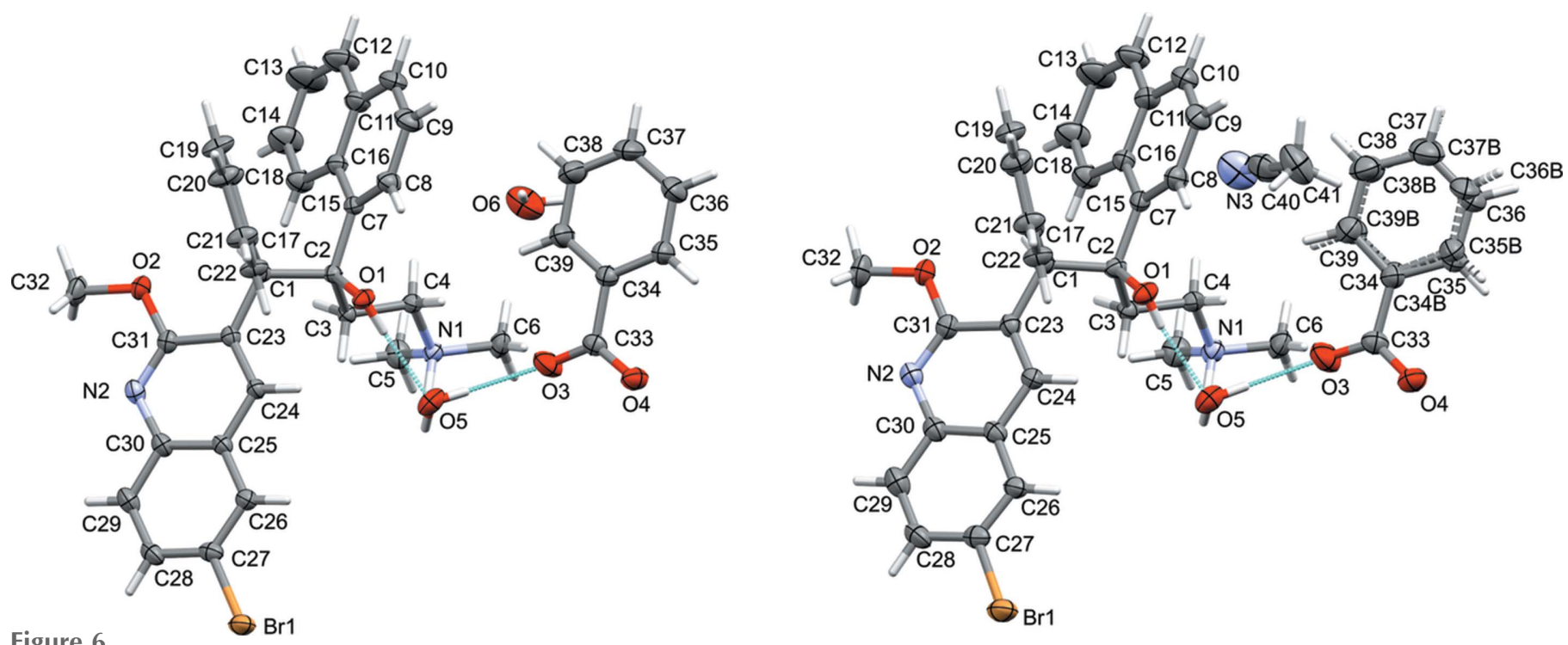

Figure 6

Single-crystal structures of benzoate salts of $\mathbf{4 a}$ (left) and $\mathbf{4 b}$ (right) (50\% probability displacement ellipsoids). Hydrogen bonds are shown as turquoise dashed lines. 
values of the acids and with the reduced basicity of the quinoline $\mathrm{N}$ atom of bedaquiline, compared to ordinary pyridine. The first $\mathrm{p} K_{a}$ of fumaric acid is 3.053 , the second is 4.494, and that of benzoic acid is 4.202 (Martell \& Smith, 1976), which are easily sufficient to protonate the amine moiety of bedaquiline [the $\mathrm{p} K_{a}$ of trialkylammonium ions are around 10-11 (Bioquest, 2020)]. The $\mathrm{p} K_{a}$ of the conjugated acid of bedaquiline protonated at the quinoline $\mathrm{N}$ atom is not reported but can be estimated from the known values for quinoline, pyridine, and 2-methoxypyridine, which are 4.9, 5.23, and 3.06, respectively (Bioquest, 2020). Methoxy substitution in the 2-position to the $\mathrm{N}$ atom substantially reduces the basicity of the $\mathrm{N}$ atom (the $\mathrm{p} K_{a}$ of the conjugated acid drops by 2.17 between pyridine and 2-methoxypyridine). Assuming other effects to be negligible yields a $\mathrm{p} K_{a}$ value of 2.73 for 2-methoxyquinolinium. The quinoline $\mathrm{N}$ atom of bedaquiline is thus not basic enough to be protonated by medium-strength acids, such as benzoic or fumaric acid, in agreement with the findings from the crystal structures for $\mathbf{2}$, $\mathbf{4 a}$, and $\mathbf{4 b}$. Stronger acids, such as mineral acids or maleic acid (first $\mathrm{p} K_{a}$ is 1.94; Bioquest, 2020), might be able to double protonate bedaquiline if used in sufficient excess. Experiments in this direction are ongoing in our laboratories.

All three salts do crystallize in the chiral monoclinic space group $P 2_{1}$. The core of the bedaquilinium cation in the three structures is formed by the ethylene moiety of atoms $\mathrm{C} 1$ and $\mathrm{C} 2$, from which the four major substituents radiate off: the phenyl ring and the bromo(methoxy)quinoline group from $\mathrm{C} 1$, and the naphthyl and (dimethylamino)ethyl fragments from
$\mathrm{C} 2$. The hydroxy group is also attached to $\mathrm{C} 2$, while $\mathrm{C} 1$ also carries a single $\mathrm{H}$ atom. Atoms $\mathrm{C} 1$ and $\mathrm{C} 2$ are also the chiral centers of the bedaquilinium cation, which were modeled to have $R$ and $S$ chiralities, respectively, in agreement with the reported absolute structure for free base bedaquiline (Petit $e t$ al., 2007). The Flack parameters refined to -0.014 (1) for $\mathbf{2}$, and to 0.006 (3) and 0.004 (8) for $\mathbf{4 a}$ and $\mathbf{4 b}$, respectively, confirming that the crystals were enantiopure.

The arrangement of anions and cations, and packing interactions, however, vary substantially between the fumarate and the two benzoate salts. The fumarate salt crystallized in an anhydrous and unsolvated form. Two crystallographically unique ion pairs are present in the lattice (i.e. $Z^{\prime}=2$ for compound 2). The structure obtained agrees with the reported powder patterns of commercially available bedaquilinium fumarate (see Fig. 7 for a Rietveld refinement plot).

The two newly isolated benzoate salts are distinctly different from 2, both being solvates, but they are very similar to each other, and are indeed isomorphous (the acetonitrile solvate was solved by isomorphous replacement from the hydrate). Both structures feature one tightly bound water molecule (atom O5). A second interstitial site does, however, differ between the two phases. In $\mathbf{4 a}$, it is occupied by a second water molecule, which is partially occupied. In $\mathbf{4 b}$, on the other hand, this site is partially occupied by a disordered acetonitrile molecule, which in turn induces disorder in the phenyl ring of the benzoate anion [see Refinement (\$2.2.2) for disorder details].

The ethane backbone of the bedaquiline core gives the cations a three-dimensional (3D) shape, but the individual

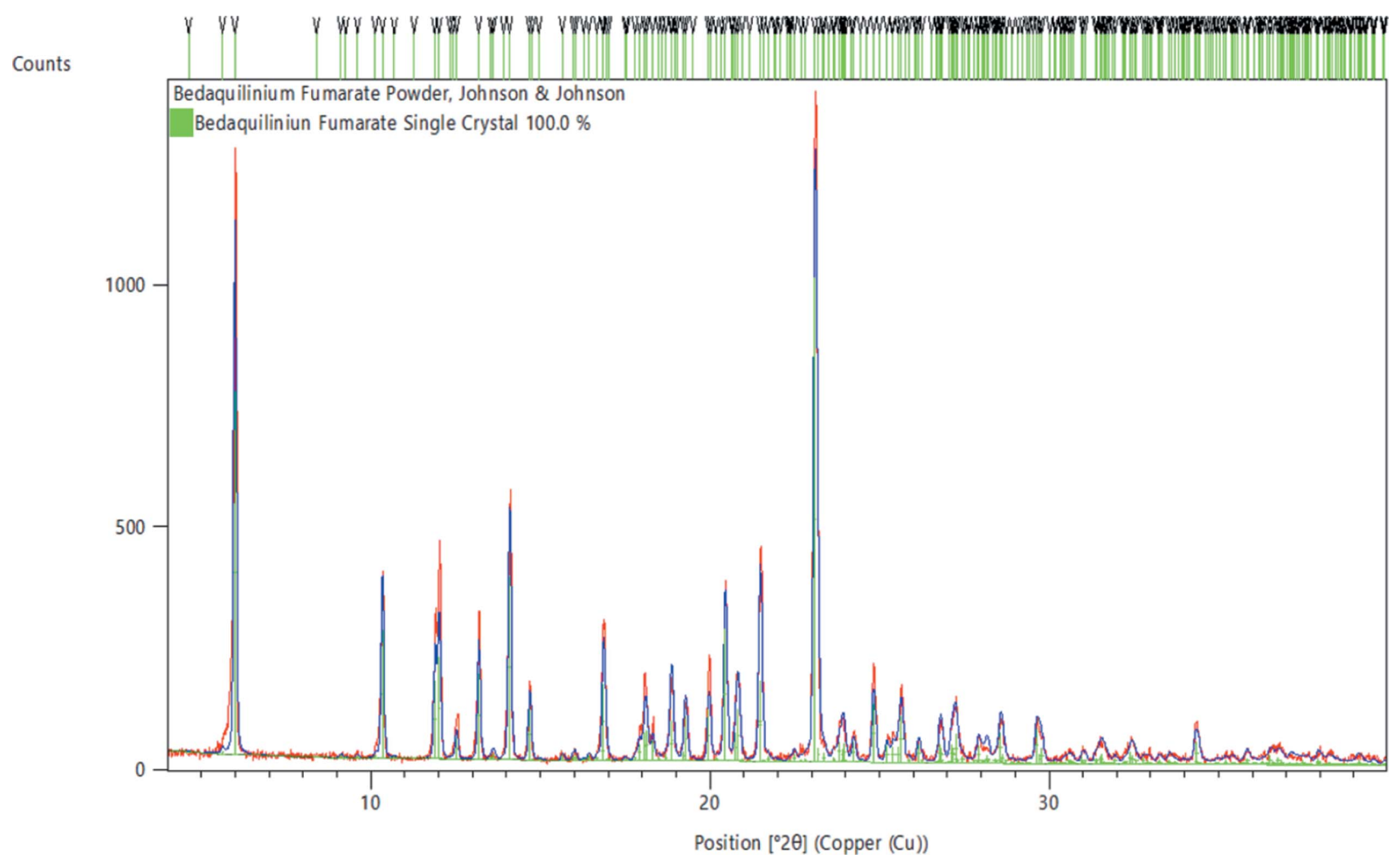

Figure 7

Powder XRD pattern (ambient temperature) of bedaquilinium fumarate (Johnson \& Johnson) with a Rietveld refinement fit against the single-crystal structure of 2 . The room-temperature unit-cell parameters refined to $a=16.5879(2), b=10.4952(8), c=20.183(2) \AA, \beta=109.238(2)^{\circ}$ and $V=$ 3317.4 (4) $\AA^{3}$. Rietveld fits for $\mathbf{4 a}$ and $\mathbf{4 b}$ are given in the supporting information. 
Table 2

Selected torsion angles $\left({ }^{\circ}\right)$ for free base bedaquiline $\mathbf{1}$, fumarate salt $\mathbf{2}$, and benzoate salts $\mathbf{4 a}$ and $\mathbf{4 b}$.

\begin{tabular}{|c|c|c|c|c|}
\hline & $\mathbf{1}^{a, b}$ & $\mathbf{2}^{b}$ & $4 a^{a}$ & $4 b^{a}$ \\
\hline$\tau$ plane 1 versus plane $2^{c}$ & $73.29(7), 81.37(7)$ & $85.33(9), 86.02(8)$ & $77.31(6)$ & $76.2(1)$ \\
\hline$\tau$ plane 2 versus plane $3^{c}$ & $86.13(8) 76.95(7)$ & 89.7 (1), $89.74(9)$ & $45.62(6)$ & $44.2(1)$ \\
\hline$\tau$ plane 1 versus plane $3^{c}$ & $14.0(1), 8.16(9)$ & $8.71(3), 16.67(4)$ & $37.50(6)$ & $36.7(1)$ \\
\hline$\tau \mathrm{C} 2-\mathrm{C} 3-\mathrm{C} 4-\mathrm{N} 1$ & $58.7(4), 63.0(4)$ & $137.2(2), 133.7(2)$ & $164.04(15)$ & $165.5(3)$ \\
\hline$\tau \mathrm{C} 17-\mathrm{C} 1-\mathrm{C} 2-\mathrm{C} 3$ & $169.0(3), 178.8(3)$ & $169.7(2), 171.38(19)$ & $174.44(14)$ & $174.1(3)$ \\
\hline
\end{tabular}

Notes: $(a)$ measured at $150 \mathrm{~K} ;(b) Z^{\prime}=2 ;(c)$ plane 1 = 6-bromo-2-methoxyquinoline, plane 2 = phenyl, and plane $3=$ naphthyl.

Table 3

Hydrogen-bond geometry $\left(\AA{ }^{\circ}\right)$ for $\mathbf{1}$.

\begin{tabular}{lllll}
\hline$D-\mathrm{H} \cdots A$ & $D-\mathrm{H}$ & $\mathrm{H} \cdots A$ & $D \cdots A$ & $D-\mathrm{H} \cdots A$ \\
\hline O1-H1O $\cdots \mathrm{N} 1$ & 0.84 & 1.94 & $2.696(4)$ & 150 \\
$\mathrm{C} 1-\mathrm{H} 1 \cdots \mathrm{O} 2$ & 1.00 & 2.24 & $2.763(4)$ & 111 \\
O3-H3O $\cdots$ N3 & 0.84 & 1.93 & $2.685(4)$ & 149 \\
C33-H33 $\cdots$ O4 & 1.00 & 2.23 & $2.773(4)$ & 112 \\
\hline
\end{tabular}

aromatic fragments remain planar. Similar to $\mathbf{3}$, the 6-bromo2-methoxyquinoline moiety is planar, with the methoxy group pointing away from the core of the cation. The r.m.s. deviations from planarity are 0.1127 and 0.1363 for the two cations in $2\left(Z^{\prime}=2\right), 0.1019 \AA$ in $\mathbf{4 a}$, and $0.0922 \AA$ in $\mathbf{4 b}$.

The ethane backbone and the malleable ethylamine fragment gives the bedaquilinium cations a high degree of conformational flexibility. Differing packing arrangements, induced by the presence (or absence) of varying anions and solvent molecules, led to a landscape of conformations observed for the cations in $\mathbf{2}, \mathbf{4 a}$, and $\mathbf{4 b}$, as well as free base bedaquiline $\mathbf{1}$. The dihedral angles between the mean planes of the 6-bromo-2-methoxy-quinoline fragment (plane 1), the phenyl ring (plane 2), and the naphthyl group (plane 3), as well as the torsion angles along the ethane backbone and the ethylamine fragment, are given in Table 2. Besides the obvious similarities between the values for isomorphous $\mathbf{4 a}$ and $\mathbf{4 b}$, no general trend is observed. The conformations vary not only between the four structures, but even between independent molecules within the same structure (both free base $\mathbf{1}$ and fumarate $\mathbf{2}$ are $Z^{\prime}=2$ structures). The two $\mathrm{C} 1-\mathrm{C} 2-\mathrm{C} 3-\mathrm{C} 4$ torsion angles in fumarate salt $\mathbf{2}$, for example, are -63.8 (3) and $174.92(19)^{\circ}$, which are distinctly different from each other. However, some similarities can be observed: the interplanar angles between the phenyl and 6-bromo-2-methoxyquinoline planes are between 70 and $90^{\circ}$ in all structures, and the torsion angles involving the ethane backbone and the ipso phenyl atom $(\mathrm{C} 17-\mathrm{C} 1-\mathrm{C} 2-\mathrm{C} 3)$ are close to antiperiplanar ('trans') in all the compounds. No other similarities common to all four structures can be found and the overall trend is one of pronounced flexibility and variability.

While the geometries and conformations in the four bedaquiline structures do not follow any general trend, there are some differences between the geometries of free base bedaquiline and its salts that can be rationalized. Directional interactions that differ between the free base and the salts play a major role. In bedaquiline, only one actual hydrogen bond is
Table 4

Hydrogen-bond geometry $\left(\AA,^{\circ}\right)$ for 2.

\begin{tabular}{lllll}
\hline$D-\mathrm{H} \cdots A$ & $D-\mathrm{H}$ & $\mathrm{H} \cdots A$ & $D \cdots A$ & $D-\mathrm{H} \cdots A$ \\
\hline $\mathrm{O} 1 A-\mathrm{H} 1 A B \cdots \mathrm{O} 4 A$ & $0.86(4)$ & $1.88(4)$ & $2.699(3)$ & $159(4)$ \\
$\mathrm{O} 5 A-\mathrm{H} 5 A \cdots \mathrm{O} 4 B$ & $1.04(4)$ & $1.58(4)$ & $2.603(3)$ & $169(4)$ \\
$\mathrm{N} 1 A-\mathrm{H} 1 A \mathrm{O} \cdots \mathrm{O} A$ & $0.98(4)$ & $1.68(4)$ & $2.641(3)$ & $163(3)$ \\
$\mathrm{C} 1 A-\mathrm{H} 1 A \cdots \mathrm{O} 2 A$ & 1.00 & 2.23 & $2.767(3)$ & 112 \\
$\mathrm{C} 3 A-\mathrm{H} 3 A B \cdots \mathrm{Br} 1 B^{\mathrm{i}}$ & 0.99 & 3.01 & $3.850(3)$ & 143 \\
$\mathrm{C} 5 A-\mathrm{H} 5 A A \cdots \mathrm{O} 1 A^{\mathrm{ii}}$ & 0.98 & 2.58 & $3.501(3)$ & 157 \\
$\mathrm{C} 5 A-\mathrm{H} 5 A C \cdots \mathrm{Br} 1 B^{\mathrm{i}}$ & 0.98 & 2.82 & $3.612(3)$ & 139 \\
$\mathrm{C} 26 A-\mathrm{H} 26 A \cdots \mathrm{O} 5 B^{\mathrm{iii}}$ & 0.95 & 2.54 & $3.459(4)$ & 163 \\
$\mathrm{C} 34 A-\mathrm{H} 34 \cdots \cdots 5 B^{\text {iii }}$ & 0.95 & 2.52 & $3.379(3)$ & 151 \\
$\mathrm{O} 1 B-\mathrm{H} 1 B \cdots \mathrm{O} 4 B^{\text {iv }}$ & $0.89(4)$ & $1.88(4)$ & $2.741(3)$ & $160(4)$ \\
$\mathrm{O} 6 B-\mathrm{H} 6 B \cdots \mathrm{O} 4 A^{\mathrm{v}}$ & $0.86(5)$ & $1.80(5)$ & $2.625(3)$ & $159(4)$ \\
$\mathrm{N} 1 B-\mathrm{H} 1 B \mathrm{~N} \cdots \mathrm{O} 3 B^{\text {iv }}$ & $0.85(4)$ & $1.83(4)$ & $2.632(3)$ & $158(4)$ \\
$\mathrm{C} 5 B-\mathrm{H} 5 B A \cdots \mathrm{O} 1 B^{\mathrm{vi}}$ & 0.98 & 2.42 & $3.337(4)$ & 156 \\
$\mathrm{C} 5 B-\mathrm{H} 5 B B \cdots \mathrm{Br} 1 A$ & 0.98 & 2.88 & $3.468(3)$ & 119 \\
$\mathrm{C} 5 B-\mathrm{H} 5 B B \cdots \mathrm{O} 6 A$ & 0.98 & 2.42 & $3.287(4)$ & 148 \\
\hline
\end{tabular}

Symmetry codes: (i) $x+1, y, z+1$; (ii) $-x+2, y+\frac{1}{2},-z+1$; (iii) $x, y-1, z$; (iv) $-x+1, y-\frac{1}{2},-z ;$ (v) $x, y+1, z ;(\mathrm{vi})-x+1, y+\frac{1}{2},-z$.

present, and this is an intramolecular $\mathrm{O}-\mathrm{H} \cdots \mathrm{N}$ hydrogen bond (Table 3). It induces the amine $\mathrm{N}$ atoms to turn towards the hydroxy group, thus enforcing a gauche geometry of the (dimethylamino)ethyl group (see torsion angle $\mathrm{C} 2-\mathrm{C} 3-$ $\mathrm{C} 4-\mathrm{N} 1$ in Table 2). Intermolecular interactions between molecules in $\mathbf{1}$ are limited to weaker and less directional interactions, specifically $\mathrm{Br} \cdots \mathrm{Br}$ interactions and $\pi$-stacking, which had been discussed in detail by Petit et al. (2007). In the fumarate and benzoate salts $\mathbf{2}$ and $\mathbf{4}$, the opposite is observed. The amine moiety, being protonated, is unable to act as the acceptor for an $\mathrm{O}-\mathrm{H} \cdots \mathrm{N}$ hydrogen bond, and it takes up the role of a hydrogen-bond donor towards the benzoate or fumarate anions. This releases the amine from the gauche conformation enforced by the intramolecular $\mathrm{O}-\mathrm{H} \cdots \mathrm{N}$ hydrogen bond in $\mathbf{1}$, and the ethylamine instead extends into a more relaxed conformation, approaching anti in $\mathbf{4}$ and close to eclipsed in 2 (see torsion angle $\mathrm{C} 2-\mathrm{C} 3-\mathrm{C} 4-\mathrm{N} 1$ in Table 2). The hydroxy groups in the salts are freed up to also form intermolecular hydrogen bonds. In 4, they hydrogen bond with the fully occupied water molecule, and in $\mathbf{2}$ with a fumarate carboxylate group. The water molecule in $\mathbf{4}$ in turn extends hydrogen bonds to the $\mathrm{O}$ atoms of two neighboring benzoate anions, and the fumarate anions in $\mathbf{2}$ are involved in a series of hydrogen bonds among each other, forming an infinite chain of hydrogen-bonded anions along the $b$-axis direction (Fig. 8). Thus, one intramolecular hydrogen bond in free base bedaquiline $\mathbf{1}$ is converted into a whole series of strong intermolecular hydrogen bonds (Tables 4, 5 and 6), creating for the 
Table 5

Hydrogen-bond geometry $\left(\AA{ }^{\circ}\right)$ for $\mathbf{4 a}$.

\begin{tabular}{lllll}
\hline$D-\mathrm{H} \cdots A$ & $D-\mathrm{H}$ & $\mathrm{H} \cdots A$ & $D \cdots A$ & $D-\mathrm{H} \cdots A$ \\
\hline $\mathrm{O} 1-\mathrm{H} 1 B \cdots \mathrm{O} 5$ & 0.84 & 1.87 & $2.681(2)$ & 164 \\
$\mathrm{O} 5-\mathrm{H} 5 D \cdots \mathrm{O} 4^{\mathrm{i}}$ & $0.90(4)$ & $1.85(4)$ & $2.724(2)$ & $163(3)$ \\
$\mathrm{O} 5-\mathrm{H} 5 E \cdots \mathrm{O} 3$ & $0.77(3)$ & $1.96(3)$ & $2.724(2)$ & $172(4)$ \\
$\mathrm{O} 6-\mathrm{H} 6 E \cdots \mathrm{N} 2$ & 0.87 & 2.38 & $3.148(13)$ & 147 \\
N1-H1 $\cdots \mathrm{O} 4^{\mathrm{i}}$ & 1.00 & 1.64 & $2.643(2)$ & 178 \\
$\mathrm{C} 5-\mathrm{H} 5 C \cdots \mathrm{Br} 1^{\text {ii }}$ & 0.98 & 3.09 & $3.910(2)$ & 142 \\
$\mathrm{C} 6-\mathrm{H} 6 A \cdots \mathrm{O} 3$ & 0.98 & 2.53 & $3.465(3)$ & 161 \\
$\mathrm{C} 6-\mathrm{H} 6 B \cdots 6^{\text {iii }}$ & 0.98 & 2.28 & $3.249(13)$ & 169 \\
$\mathrm{C} 28-\mathrm{H} 28 \cdots \mathrm{O}^{\text {ii }}$ & 0.95 & 2.59 & $3.421(3)$ & 146 \\
\hline
\end{tabular}

Symmetry codes: (i) $-x+1, y-\frac{1}{2},-z$; (ii) $-x+2, y-\frac{1}{2},-z$; (iii) $x-1, y, z$.

salts a much stronger cohesion between neighboring structural entities than what was observed in the free base structure. Weaker interactions that dominate for bedaquiline, such as $\mathrm{Br} \cdots \mathrm{Br}$ halogen bonds and $\pi$-stacking, are not observed for
Table 6

Hydrogen-bond geometry $\left(\AA,^{\circ}\right)$ for $\mathbf{4 b}$.

\begin{tabular}{lllll}
\hline$D-\mathrm{H} \cdots A$ & $D-\mathrm{H}$ & $\mathrm{H} \cdots A$ & $D \cdots A$ & $D-\mathrm{H} \cdots A$ \\
\hline $\mathrm{O} 1-\mathrm{H} 1 B \cdots \mathrm{O} 5$ & 0.77 & 1.94 & $2.691(4)$ & 163 \\
$\mathrm{O} 5-\mathrm{H} 5 D \cdots \mathrm{O} 4^{\mathrm{i}}$ & $0.86(3)$ & $1.89(3)$ & $2.738(4)$ & $168(6)$ \\
$\mathrm{O} 5-\mathrm{H} 5 E \cdots \mathrm{O} 3$ & $0.82(3)$ & $1.92(3)$ & $2.734(4)$ & $172(6)$ \\
$\mathrm{N} 1-\mathrm{H} 1 \cdots \mathrm{O} 4^{\mathrm{i}}$ & 1.00 & 1.62 & $2.619(4)$ & 178 \\
$\mathrm{C} 5-\mathrm{H} 5 C \cdots \mathrm{Br} 1^{\mathrm{ii}}$ & 0.98 & 3.13 & $3.964(4)$ & 144 \\
$\mathrm{C} 6-\mathrm{H} 6 A \cdots \mathrm{O} 3$ & 0.98 & 2.63 & $3.562(6)$ & 159 \\
$\mathrm{C} 28-\mathrm{H} 28 \cdots \mathrm{O} 5^{\mathrm{ii}}$ & 0.95 & 2.66 & $3.498(5)$ & 148 \\
\hline
\end{tabular}

Symmetry codes: (i) $-x+1, y-\frac{1}{2},-z$; (ii) $-x+2, y-\frac{1}{2},-z$.

the salts, but the strong hydrogen bonds are augmented by a range of other not-as-strong directional interactions, such as $\mathrm{C}-\mathrm{H} \cdots \mathrm{O}$ and $\mathrm{C}-\mathrm{H} \cdots \mathrm{Br}$ hydrogen bonds (see hydrogenbond Tables 4, 5, and 6 for a full listing).

In the acetonitrile solvate $\mathbf{4 b}$, an $\mathrm{H}$ atom of the naphthyl group interacts in a $\mathrm{C}-\mathrm{H} \cdots \mathrm{N}$ hydrogen-like bond with the
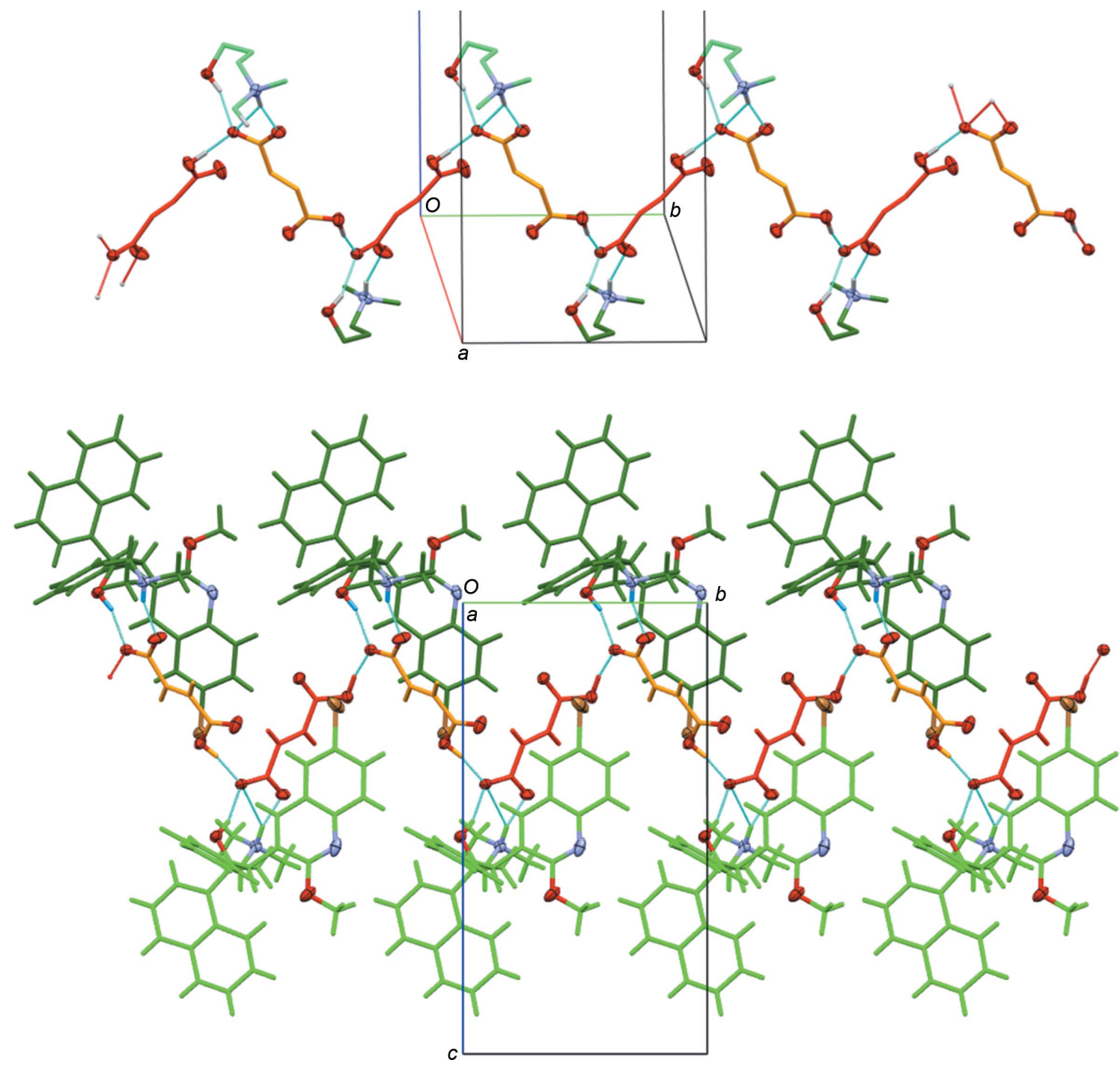

Figure 8

Hydrogen-bonding pattern in 2. (Top) Chains along $b$ created by hydrofumarate anions. For clarity, only the 3-dimethylazaniumyl-1-hydroxypropyl fragments of the cations are shown, and $\mathrm{H}$ atoms not involved in hydrogen bonds have been omitted. (Bottom) Segment of an entire ribbon, including full cations. Color coding: $\mathrm{O}, \mathrm{N}$, and $\mathrm{Br}$ atoms are shown with $50 \%$ probability displacement ellipsoids, with all other atoms in capped-stick mode and color coded by the symmetry equivalence of their anion and cation ( $A$ and $B$ cations in light and dark green, and $A$ and $B$ anions in orange and red). Hydrogen bonds are shown as turquoise and red dashed lines. 


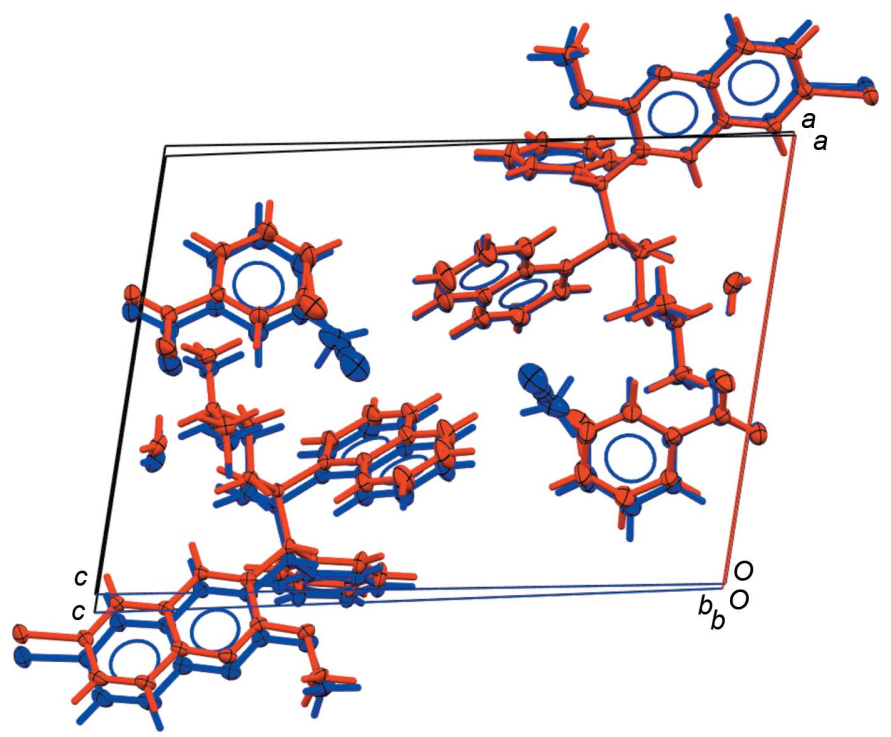

Figure 9

Least-squares overlay of the structures of $\mathbf{4 a}$ (red) and $\mathbf{4 b}$ (blue) using the non-H atoms for one of the two ion pairs of the asymmetric unit (r.m.s. fit value $=0.152$ ), ignoring water and solvent molecules, and disorder. When extending the fit to the atoms of both ion pairs of the unit cell, the r.m.s. value increases to 0.195, showing the lattice mismatch between $\mathbf{4 a}$ and $\mathbf{4 b}$.

solvent molecule, when present. In its absence, the phenyl ring of the benzoate anion relaxes towards the void created, inducing disorder for the anion [see Refinement (\$2.2.2) for details]. For the 1.17-hydrate $\mathbf{4 a}$, the partially occupied water molecule is hydrogen bonded to the pyridine $\mathrm{N}$ atom and acts as an acceptor for a $\mathrm{C}-\mathrm{H} \cdots \mathrm{O}$ interaction. There is, however, no second possible hydrogen-bond acceptor near the partially occupied water molecule, and the second water $\mathrm{H}$ atom is not involved in any recognizable interaction. This is energetically

Table 7

$150 \mathrm{~K}$ and room-temperature (RT) unit-cell dimensions for $\mathbf{1}, \mathbf{2}, \mathbf{4 a}$, and $\mathbf{4 b}$.

RT data were obtained from powder XRD patterns via Rietveld refinement (see supporting information for Rietveld plots).

\begin{tabular}{lllll}
\hline & $\mathbf{1}, 150 \mathrm{~K}$ & $\mathbf{1}, \mathrm{RT}$ & $\mathbf{2}, 150 \mathrm{~K}$ & $\mathbf{2}, \mathrm{RT}$ \\
\hline$a(\AA)$ & $11.1584(8)$ & $11.230(1)$ & $16.4556(6)$ & $16.5879(2)$ \\
$b(\AA)$ & $13.6425(14)$ & $13.766(1)$ & $10.3205(3)$ & $10.4952(8)$ \\
$c(\AA)$ & $36.061(4)$ & $36.455(3)$ & $20.1636(8)$ & $20.183(2)$ \\
$\beta\left({ }^{\circ}\right)$ & 90 & 90 & $109.1832(15)$ & $109.238(2)$ \\
$V\left(\AA^{3}\right)$ & $5489.5(9)$ & $5636(1)$ & $3234.2(2)$ & $3317.4(4)$ \\
$\rho\left(\mathrm{Mg} \mathrm{m}^{-3}\right)$ & 1.344 & 1.309 & 1.379 & 1.344 \\
$R_{\text {wp }}(\%)$ & & 19.99 & & 20.48 \\
$R_{\exp }(\%)$ & & 15.67 & & 13.27 \\
$S$ & & 1.27 & & 1.54
\end{tabular}

\begin{tabular}{llllll}
\hline & $\mathbf{4 a}, 150 \mathrm{~K}$ & $\mathbf{4 a}, \mathrm{RT}$ & $\mathbf{4 b}, 150 \mathrm{~K}$ & \multicolumn{2}{c}{$\mathbf{4 b}, \mathrm{RT}^{a}$} \\
\hline$a(\AA)$ & $12.6384(5)$ & $12.7267(4)$ & $12.8661(8)$ & $12.720(1)$ & $12.7242(8)$ \\
$b(\AA)$ & $7.9259(3)$ & $8.0157(3)$ & $8.0386(5)$ & $8.0094(5)$ & $8.0116(6)$ \\
$c(\AA)$ & $17.5249(8)$ & $17.6438(7)$ & $17.4704(10)$ & $17.626(2)$ & $17.632(1)$ \\
$\beta\left({ }^{\circ}\right)$ & $99.8450(17)$ & $99.7928(6)$ & $101.093(3)$ & $99.836(2)$ & $99.813(1)$ \\
$V\left(\AA^{3}\right)$ & $1729.63(12)$ & $1773.7(1)$ & $1773.13(19)$ & $1769.4(3)$ & $1771.2(2)$ \\
$\rho\left(\mathrm{Mg} \mathrm{m}^{-3}\right)$ & 1.342 & 1.309 & 1.360 & 1.311 & 1.361 \\
$R_{\text {wp }}(\%)$ & & 5.57 & & 30.65 & 28.65 \\
$R_{\text {exp }}(\%)$ & & 10.78 & & 10.50 & 14.26 \\
$S$ & & 1.94 & & 2.12 & 2.01 \\
\hline
\end{tabular}

Note: (a) data from two independent samples and Rietveld refinements. unfavorable, which helps to explain why this position is occupied less than $20 \%$ of the time [the refined value is $16.6(7) \%]$, while the other solvent water molecule, tightly hydrogen bonded on all sides, is fully occupied. Lack of space appears to be no issue, as the larger acetonitrile molecule in $\mathbf{4 b}$ has a higher occupancy of around three-quarters [refined value 74.2 (7)\%]. Kinetic factors during crystal growth (a lack of water molecules during crystallization from mostly dry solvents for $\mathbf{4 a}$, but no lack of acetonitrile molecules for $\mathbf{4 b}$ ) seem to dominate. The presence or absence of either water or acetonitrile in this void does not appear to hinder continuation of crystal growth, while this cannot be said for the tightly incorporated $\mathrm{O} 5$ water molecule present in both structures, which appears to be essential for the formation of this structure. The larger size of acetonitrile versus water, and the low prevalence of the second water molecule in $\mathbf{4 a}$, lead to a slightly larger unit-cell volume for $\mathbf{4 b}$ compared to $\mathbf{4 a}$, i.e. 1773.13 (19) versus 1729.63 (12) $\AA^{3}$. The visually most obvious difference between the two structures is the difference in the $\beta$ angle, which is expanded by slightly more than $1^{\circ}$ in $\mathbf{4 b}$, leading to a significant offset between atoms in the two structures when the unit cells are overlaid (Fig. 9). The same is evident when comparing the powder patterns of $\mathbf{4 a}$ and $\mathbf{4 b}$ simulated from the $150 \mathrm{~K}$ single-crystal data, which are distinctively different (see supporting information).

Observations based on powder XRD data indicate that the samples of $\mathbf{4 b}$ quickly desolvate, even under ambient conditions. The powder patterns of $\mathbf{4 b}$ more closely match the parameters of hydrate 4a than would be expected for acetonitrile solvate $\mathbf{4 b}$, even if the data were collected on samples exposed to air only for a few minutes prior to data collection (Table 7). Rietveld refinements of samples of $\mathbf{4 b}$ yield $\beta$ angles that match those of $4 a$ (at both room temperature and $150 \mathrm{~K}$ ), and the room-temperature volume of $\mathbf{4 b}$ is actually slightly smaller than that of $\mathbf{4 a}$, in agreement with the assumption that all the acetonitrile in the structure of 4b is lost when exposed to the atmosphere, while the solvent water molecules of $\mathbf{4 a}$, being hydrogen bound, do remain in the lattice under these conditions. These observations will be further investigated in a more detailed upcoming study, focusing on a larger series of bedaquilinium salts, including their solvation properties, thermal stability, and responses to ambient moisture.

The structure of $\mathbf{2}$, on the other hand, is solvent free. Like 4, the main intermolecular interactions are strong hydrogen bonds, already briefly discussed, with the bedaquilinium cation always acting as a hydrogen-bond donor, and the hydrofumarate anions acting as both $\mathrm{H}$ atom acceptors and donors. The quinoline $\mathrm{N}$ atom does not act as an acceptor for a hydrogen bond, in agreement with its reduced basicity, already discussed. Originating from the cation are $\mathrm{N}-\mathrm{H} \cdots \mathrm{O}$ hydrogen bonds, formed by the ammonium cations, and $\mathrm{O}-$ $\mathrm{H} \cdots \mathrm{O}$ hydrogen bonds, originating from the alcohol moieties. The $\mathrm{N}-\mathrm{H} \cdots \mathrm{O}$ and $\mathrm{O}-\mathrm{H} \cdots \mathrm{O}$ hydrogen bonds from one cation are towards the two $\mathrm{O}$ atoms of 
the same fully deprotonated carboxylate group, yielding an $R_{2}^{2}(10)$ hydrogen-bonding graph-set motif. This motif is identical for the two crystallographically independent ion pairs in 2. The remaining strong hydrogen bonds are between the hydrofumarate anions. The hydrogen-bond donors are the carboxylic acid groups of each hydrofumarate anion, while the hydrogen-bond acceptor is always the $\mathrm{O}$ atom also hydrogen bonded to the bedaquilinium alcohol group, thus tying fumarate anions together in a head-to-tail fashion, forming infinite chains that extend along the $b$-axis direction (Fig. 8). The individual hydrogen-bonding motifs for these hydrogen bonds are $D(2)$. The hydrofumarate anion chains bridge between bedaquilinium cations connecting anions and cations into ribbons that extend along the $b$-axis direction.
These strong hydrogen bonds in $\mathbf{2}$ are again augmented by a series of other not-as-strong directional interactions, such as $\mathrm{C}-\mathrm{H} \cdots \mathrm{O}$ and $\mathrm{C}-\mathrm{H} \cdots \mathrm{Br}$ hydrogen bonds (Table 4) that interconnect between ribbons to create a fully $3 \mathrm{D}$ network and structure. Segments of neighboring ribbons do also interdigitate with each other, further stabilizing the overall structure.

The arrangement of anions and cations in $4 \mathbf{a}$ and $\mathbf{4 b}$, and their connection via hydrogen-bonding interactions, follows a similar pattern to that in $\mathbf{2}$, but it is augmented by the solvate water molecules, which play a similar role as the protonated fumarate carboxylic acid groups do in $\mathbf{2}$ in connecting anions and cations with each other into infinite ribbons (Fig. 10). Bedaquilinium cations and water molecules act as hydrogen-
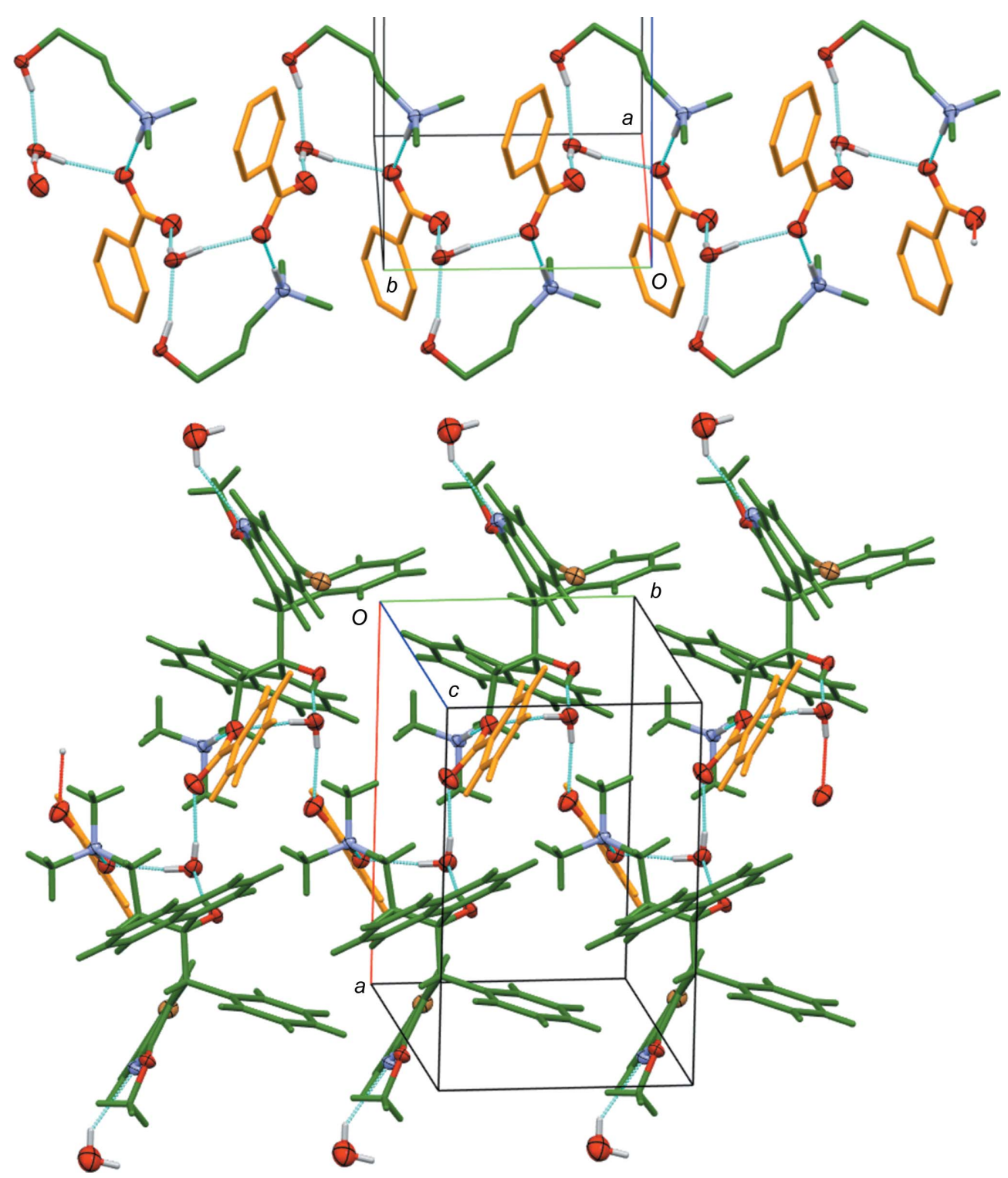

Figure 10

Hydrogen-bonding pattern in 4a. (Top) Chains along $b$ created by benzoate anions and water molecules. For clarity, only the 3-dimethylazaniumyl-1hydroxypropyl fragments of the cations are shown, and $\mathrm{H}$ atoms not involved in hydrogen bonds have been omitted. (Bottom) Segment of an entire ribbon, including cations and partially occupied water molecules. Color coding: $\mathrm{O}, \mathrm{N}$, and $\mathrm{Br}$ atoms are shown with $50 \%$ probability displacement ellipsoids, and all other atoms are in capped-stick mode and color coded by the symmetry equivalence of their anion and cation (cations in dark green and benzoate anions in orange). Hydrogen bonds are shown as turquoise and red dashed lines. 
bond donors and the benzoate anions act as hydrogen-bond acceptors. The $\mathrm{N}-\mathrm{H} \cdots \mathrm{O}$ hydrogen bond from the cation is towards one $\mathrm{O}$ atom of the benzoate carboxylate group and the $\mathrm{O}-\mathrm{H} \cdots \mathrm{O}$ hydrogen bond is towards the water molecule, which in turn is hydrogen bonded to the same benzoate $\mathrm{O}$ atom as the ammonium fragment. The $R_{2}^{2}(10)$ graph-set motif in $\mathbf{2}$ is thus converted in $\mathbf{4}$ into an $R_{3}^{3}(10)$ motif, but otherwise fulfils the same function as in $\mathbf{2}$, connecting the hydroxy and ammonium segments of one cation to the same carboxylate group. The fully occupied water molecules in $\mathbf{4}$ assume the role of the carboxylic acid groups in $\mathbf{2}$, acting as bridges between anions $\left[C_{2}^{2}(6)\right.$ hydrogen-bonding motif], creating infinite benzoate-water chains that extend along the $b$-axis direction. Wrapped around these chains, and connected to them via $\mathrm{O}-$ $\mathrm{H} \cdots \mathrm{O}$ and $\mathrm{N}-\mathrm{H} \cdots \mathrm{O}$ hydrogen bonds, are the bedaquiline cations, thus creating wider ribbons of cations, anions, and solvent water molecules (Fig. 10), emulating the pattern already observed for $\mathbf{2}$. The partially occupied water molecules are located on the outer rim of the ribbon, hydrogen bonded to the quinoline $\mathrm{N}$ atom but not bridging or connecting between any structural entities.

The strong intermolecular interactions present in $\mathbf{4 a}$ and $\mathbf{4 b}$ do compensate for the presence of the only partially or not at all filled voids present, and the packing efficiency for the salts of $\mathbf{4}$ is comparable to that of free base bedaquiline. Indeed, the density for the 1.17 -hydrate is, at $1.342 \mathrm{Mg} \mathrm{m}^{-3}$, virtually identical to that of the free base of $1.344 \mathrm{Mg} \mathrm{m}^{-3}$ (both measured at $150 \mathrm{~K}$ ), and the acetonitrile solvate is, at $1.360 \mathrm{Mg} \mathrm{m}^{-3}$, even denser than the solvent-free base. The best packing efficiency is, however, observed for fumarate salt $\mathbf{2}$, featuring a multitude of strong hydrogen bonds, and having neither incorporated solvent molecules nor unoccupied void space. Its density, at $150 \mathrm{~K}$, is $1.379 \mathrm{Mg} \mathrm{m}^{-3}$ (Table 7).

\section{Acknowledgements}

This material is based on work supported by the National Science Foundation (NSF) through the Major Research Instrumentation Program, which provided funding for the single-crystal X-ray diffractometer.

\section{Funding information}

Funding for this research was provided by: National Science Foundation, Directorate for Mathematical and Physical
Sciences (grant No. 1625543 to MZ); Bill and Melinda Gates Foundation (grant No. INV-017799 to SRB).

\section{References}

Bioquest (2020). AAT Bioquest $p K_{a}$ and $p K_{b}$ Reference Table, https:// www.aatbio.com/data-sets/pka-and-pkb-reference-table, accessed August 21, 2020.

Brigden, G., Hewison, C. \& Varaine, F. (2015). Infect. Drug Resist. 8, 367-378.

Bruker (2019). APEX3 and SAINT. Bruker AXS Inc., Madison, Wisconsin, USA.

Colthup, N. B., Daly, L. H. \& Wiberley, S. E. (1990). In Introduction to Infrared and Raman Spectroscopy, 3rd ed. New York: Academic Press Inc.

Groom, C. R., Bruno, I. J., Lightfoot, M. P. \& Ward, S. C. (2016). Acta Cryst. B72, 171-179.

Hegyi, J. F. A. L., Aelterman, W. A. A., Lang, Y. L., Stokbroekx, S. C. M., Leys, C., Van Remoortere, P. J. M. \& Faure, A. (2013). US Patent 8546 428, Janssen Pharmaceuticals, USA.

Hübschle, C. B., Sheldrick, G. M. \& Dittrich, B. (2011). J. Appl. Cryst. 44, 1281-1284.

Kojima, T., Tsutsumi, S., Yamamoto, K., Ikeda, Y. \& Moriwaki, T. (2010). Int. J. Pharm. 399(1-2), 52-59.

Krause, L., Herbst-Irmer, R., Sheldrick, G. M. \& Stalke, D. (2015). J. Appl. Cryst. 48, 3-10.

Macrae, C. F., Sovago, I., Cottrell, S. J., Galek, P. T. A., McCabe, P., Pidcock, E., Platings, M., Shields, G. P., Stevens, J. S., Towler, M. \& Wood, P. A. (2020). J. Appl. Cryst. 53, 226-235.

Martell, A. E. \& Smith, R. M. (1976). In Critical Stability Constants, Vols. 1-4. New York: Plenum Press.

PANalytical (2015). Data Collector (XRD data collection software, Version 5.3.0.62) and HighScore (Version 4.5). PANalytical BV, Almelo, The Netherlands.

Parsons, S., Flack, H. D. \& Wagner, T. (2013). Acta Cryst. B69, 249 259.

Petit, S., Coquerel, G., Meyer, C. \& Guillemont, J. (2007). J. Mol. Struct. 837, 252-256.

Rombouts, J. A., Veenboer, R. P., Villellas, C., Lu, P., Ehlers, A. W., Andries, K., Koul, A., Lill, H., Ruijter, E., Orru, R. V. A., Lammertsma, K., Bald, D. \& Slootweg, J. C. (2016). RSC Adv. 6, 108708-108716.

Sheldrick, G. M. (2008). Acta Cryst. A64, 112-122.

Sheldrick, G. M. (2015). Acta Cryst. C71, 3-8.

Westrip, S. P. (2010). J. Appl. Cryst. 43, 920-925.

Zook, H. D., March, J. \& Smith, D. F. (1959). J. Am. Chem. Soc. 81, 1617-1620.

Zvatora, P., Dammer, O., Krejcik, L., Zvonicek, V. \& Hert, J. (2016). Int. Patent WO/2016/198031A1, Zentiva, Czech Republic.

Zvatora, P., Dammer, O., Ridvan, L., Lustig, P., Pekarek, T., Stefco, M., Krejcik, L. \& Tkadlecova, M. (2016). Int. Patent WO/2016/ 058564, Zentiva, Czech Republic. 


\section{supporting information}

Acta Cryst. (2020). C76, 1010-1023 [https://doi.org/10.1107/S2053229620013455]

\section{Crystal structures of salts of bedaquiline}

\section{Mercy Okezue, Daniel Smith, Matthias Zeller, Stephen R. Byrn, Pamela Smith, Susan Bogandowich-Knipp, Dale K. Purcell and Kari L. Clase}

\section{Computing details}

For all structures, data collection: APEX3 (Bruker, 2019); cell refinement: SAINT (Bruker, 2019); data reduction: SAINT (Bruker, 2019); program(s) used to solve structure: SHELXS97 (Sheldrick, 2008); program(s) used to refine structure: SHELXL2018 (Sheldrick, 2015) and shelXle (Hübschle et al., 2011); molecular graphics: Mercury (Macrae et al., 2020); software used to prepare material for publication: publCIF (Westrip, 2010).

1-(6-Bromo-2-methoxyquinolin-3-yl)-4-(dimethylamino)- 2-(naphthalen-1-yl)-1-phenyllbutan-2-ol (1)

Crystal data

$\mathrm{C}_{32} \mathrm{H}_{31} \mathrm{BrN}_{2} \mathrm{O}_{2}$

$M_{r}=555.50$

Orthorhombic, $P 22_{1} 2_{1} 2_{1}$

$a=11.1584(8) \AA$

$b=13.6425(14) \AA$

$c=36.061(4) \AA$

$V=5489.5(9) \AA^{3}$

$Z=8$

$F(000)=2304$

\section{Data collection}

Bruker D8 Quest

diffractometer with PhotonII charge-integrating pixel array detector (CPAD)

Radiation source: fine focus sealed tube X-ray source

Triumph curved graphite crystal monochromator

Detector resolution: 7.4074 pixels $\mathrm{mm}^{-1}$ $\omega$ and phi scans
$D_{\mathrm{x}}=1.344 \mathrm{Mg} \mathrm{m}^{-3}$

Mo $K \alpha$ radiation, $\lambda=0.71073 \AA$

Cell parameters from 9979 reflections

$\theta=2.4-28.7^{\circ}$

$\mu=1.53 \mathrm{~mm}^{-1}$

$T=150 \mathrm{~K}$

Plate, colourless

$0.21 \times 0.13 \times 0.05 \mathrm{~mm}$

Absorption correction: multi-scan

(SADABS2016; Krause et al., 2015)

$T_{\min }=0.603, T_{\max }=0.747$

66520 measured reflections

17893 independent reflections

12296 reflections with $I>2 \sigma(I)$

$R_{\text {int }}=0.052$

$\theta_{\max }=33.2^{\circ}, \theta_{\min }=2.3^{\circ}$

$h=-14 \rightarrow 17$

$k=-20 \rightarrow 17$

$l=-55 \rightarrow 52$

Primary atom site location: structure-invariant direct methods

Secondary atom site location: difference Fourier map

Hydrogen site location: inferred from neighbouring sites

$\mathrm{H}$-atom parameters constrained 
$w=1 /\left[\sigma^{2}\left(F_{\mathrm{o}}^{2}\right)+(0.0298 P)^{2}+2.4789 P\right]$

where $P=\left(F_{\mathrm{o}}^{2}+2 F_{\mathrm{c}}{ }^{2}\right) / 3$

$(\Delta / \sigma)_{\max }=0.002$

$\Delta \rho_{\max }=0.48 \mathrm{e} \AA^{-3}$

$\Delta \rho_{\min }=-0.58$ e $\AA^{-3}$
Absolute structure: Flack $x$ determined using 4397 quotients [(I+)-(I-)]/[(I+)+(I-)] (Parsons et al., 2013)

Absolute structure parameter: 0.034 (3)

Special details

Geometry. All esds (except the esd in the dihedral angle between two 1.s. planes) are estimated using the full covariance matrix. The cell esds are taken into account individually in the estimation of esds in distances, angles and torsion angles; correlations between esds in cell parameters are only used when they are defined by crystal symmetry. An approximate (isotropic) treatment of cell esds is used for estimating esds involving l.s. planes.

Fractional atomic coordinates and isotropic or equivalent isotropic displacement parameters $\left(\AA^{2}\right)$

\begin{tabular}{|c|c|c|c|c|}
\hline & $x$ & $y$ & $z$ & $U_{\text {iso }} * / U_{\text {eq }}$ \\
\hline $\mathrm{Br} 1$ & $-0.22418(4)$ & $0.59893(4)$ & $0.93403(2)$ & $0.04766(11)$ \\
\hline $\mathrm{O} 1$ & $0.3238(2)$ & 0.60084 (19) & $0.79619(6)$ & $0.0300(5)$ \\
\hline $\mathrm{H} 1 \mathrm{O}$ & 0.296203 & 0.640769 & 0.780645 & $0.045^{*}$ \\
\hline $\mathrm{O} 2$ & $0.4828(2)$ & $0.72509(17)$ & $0.90856(6)$ & $0.0311(5)$ \\
\hline N1 & $0.3008(3)$ & $0.7681(3)$ & $0.75701(9)$ & $0.0374(7)$ \\
\hline N2 & $0.2968(2)$ & $0.7233(2)$ & $0.93579(8)$ & $0.0289(6)$ \\
\hline $\mathrm{C} 1$ & $0.4337(3)$ & $0.5908(2)$ & $0.85326(9)$ & $0.0249(6)$ \\
\hline $\mathrm{H} 1$ & 0.511656 & 0.613215 & 0.863960 & $0.030^{*}$ \\
\hline $\mathrm{C} 2$ & 0.4218 & $0.6444(2)$ & $0.81522(9)$ & $0.0261(6)$ \\
\hline $\mathrm{C} 3$ & $0.3911(3)$ & $0.7541(2)$ & $0.81996(10)$ & $0.0287(7)$ \\
\hline $\mathrm{H} 3 \mathrm{~A}$ & 0.311884 & 0.760086 & 0.832083 & $0.034^{*}$ \\
\hline H3B & 0.451320 & 0.784829 & 0.836451 & $0.034^{*}$ \\
\hline $\mathrm{C} 4$ & $0.3885(3)$ & $0.8096(3)$ & $0.78309(10)$ & $0.0330(7)$ \\
\hline $\mathrm{H} 4 \mathrm{~A}$ & 0.368347 & 0.879127 & 0.787832 & $0.040 *$ \\
\hline H4B & 0.469185 & 0.807406 & 0.771700 & $0.040^{*}$ \\
\hline $\mathrm{C} 5$ & $0.1807(4)$ & 0.8058 (4) & $0.76442(14)$ & $0.0549(12)$ \\
\hline $\mathrm{H} 5 \mathrm{~A}$ & 0.181362 & 0.877472 & 0.762872 & $0.082 *$ \\
\hline H5B & 0.155422 & 0.785688 & 0.789317 & $0.082 *$ \\
\hline $\mathrm{H} 5 \mathrm{C}$ & 0.124585 & 0.779366 & 0.746042 & $0.082^{*}$ \\
\hline C6 & $0.3352(5)$ & $0.7891(4)$ & $0.71826(12)$ & $0.0540(12)$ \\
\hline H6A & 0.274637 & 0.761942 & 0.701453 & $0.081 *$ \\
\hline H6B & 0.413095 & 0.759038 & 0.712972 & $0.081^{*}$ \\
\hline $\mathrm{H} 6 \mathrm{C}$ & 0.340535 & 0.860126 & 0.714641 & $0.081 *$ \\
\hline $\mathrm{C} 7$ & $0.5377(3)$ & $0.6349(2)$ & $0.79184(10)$ & $0.0292(7)$ \\
\hline $\mathrm{C} 8$ & $0.5303(3)$ & $0.5879(3)$ & $0.75814(10)$ & $0.0358(8)$ \\
\hline $\mathrm{H} 8$ & 0.459085 & 0.553241 & 0.752149 & $0.043^{*}$ \\
\hline C9 & $0.6254(4)$ & $0.5894(3)$ & $0.73209(11)$ & 0.0437 (9) \\
\hline H9 & 0.617529 & 0.555399 & 0.709222 & $0.052 *$ \\
\hline $\mathrm{C} 10$ & 0.7269 (4) & 0.6388 (3) & $0.73966(12)$ & $0.0479(10)$ \\
\hline H10 & 0.788893 & 0.641601 & 0.721614 & $0.058^{*}$ \\
\hline $\mathrm{C} 11$ & $0.7426(3)$ & 0.6867 (3) & 0.77407 (13) & $0.0431(10)$ \\
\hline $\mathrm{C} 12$ & $0.8498(4)$ & $0.7363(4)$ & $0.78199(17)$ & 0.0594 (14) \\
\hline H12 & 0.910315 & 0.740322 & 0.763505 & $0.071 *$ \\
\hline $\mathrm{C} 13$ & 0.8689 (4) & $0.7790(4)$ & $0.81573(18)$ & $0.0624(14)$ \\
\hline
\end{tabular}




\begin{tabular}{|c|c|c|c|c|}
\hline H13 & 0.942439 & 0.811591 & 0.820635 & $0.075^{*}$ \\
\hline C14 & $0.7811(4)$ & $0.7748(3)$ & $0.84279(15)$ & $0.0529(11)$ \\
\hline H14 & 0.795439 & 0.803479 & 0.866381 & $0.064^{*}$ \\
\hline $\mathrm{C} 15$ & $0.6736(4)$ & 0.7297 (3) & $0.83590(12)$ & $0.0377(8)$ \\
\hline H15 & 0.613991 & 0.729114 & 0.854720 & $0.045 *$ \\
\hline $\mathrm{C} 16$ & $0.6489(3)$ & $0.6837(3)$ & $0.80140(11)$ & $0.0338(8)$ \\
\hline $\mathrm{C} 17$ & $0.4406(3)$ & $0.4791(2)$ & $0.85227(9)$ & $0.0271(6)$ \\
\hline C18 & $0.5242(3)$ & $0.4342(3)$ & 0.87547 (11) & $0.0361(8)$ \\
\hline H18 & 0.578525 & 0.473635 & 0.889212 & $0.043^{*}$ \\
\hline C19 & $0.5296(4)$ & $0.3329(3)$ & $0.87886(13)$ & $0.0455(10)$ \\
\hline H19 & 0.586943 & 0.304062 & 0.894989 & $0.055^{*}$ \\
\hline $\mathrm{C} 20$ & $0.4523(4)$ & 0.2738 & 0.85897 (14) & $0.0468(10)$ \\
\hline $\mathrm{H} 20$ & 0.455743 & 0.204480 & 0.861264 & $0.056^{*}$ \\
\hline $\mathrm{C} 21$ & $0.3706(4)$ & $0.3174(3)$ & 0.83584 (13) & $0.0453(10)$ \\
\hline $\mathrm{H} 21$ & 0.317076 & 0.277449 & 0.821984 & $0.054^{*}$ \\
\hline $\mathrm{C} 22$ & $0.3643(4)$ & $0.4183(3)$ & $0.83226(12)$ & $0.0374(8)$ \\
\hline $\mathrm{H} 22$ & 0.307030 & 0.446401 & 0.815903 & $0.045^{*}$ \\
\hline $\mathrm{C} 23$ & $0.3375(3)$ & $0.6233(2)$ & $0.88094(9)$ & $0.0255(6)$ \\
\hline $\mathrm{C} 24$ & $0.2215(3)$ & $0.5925(2)$ & $0.88015(9)$ & $0.0266(6)$ \\
\hline $\mathrm{H} 24$ & 0.196272 & 0.548059 & 0.861430 & $0.032 *$ \\
\hline $\mathrm{C} 25$ & $0.1375(3)$ & $0.6260(2)$ & $0.90703(9)$ & $0.0262(6)$ \\
\hline $\mathrm{C} 26$ & $0.0151(3)$ & $0.5984(3)$ & $0.90691(9)$ & $0.0303(6)$ \\
\hline $\mathrm{H} 26$ & -0.014665 & 0.554893 & 0.888515 & $0.036^{*}$ \\
\hline $\mathrm{C} 27$ & $-0.0594(3)$ & $0.6349(3)$ & $0.93353(11)$ & $0.0333(7)$ \\
\hline $\mathrm{C} 28$ & $-0.0190(3)$ & $0.6997(3)$ & $0.96125(10)$ & $0.0369(8)$ \\
\hline $\mathrm{H} 28$ & -0.073061 & 0.724205 & 0.979370 & $0.044 *$ \\
\hline $\mathrm{C} 29$ & $0.0990(3)$ & $0.7270(3)$ & $0.96179(10)$ & $0.0342(8)$ \\
\hline $\mathrm{H} 29$ & 0.126805 & 0.770477 & 0.980477 & $0.041 *$ \\
\hline $\mathrm{C} 30$ & 0.1804 & $0.6910(2)$ & $0.93480(9)$ & $0.0279(6)$ \\
\hline C 31 & $0.3688(3)$ & $0.6911(2)$ & $0.90978(9)$ & $0.0260(6)$ \\
\hline C32 & $0.5181(3)$ & $0.7945(3)$ & $0.93649(11)$ & $0.0380(8)$ \\
\hline $\mathrm{H} 32 \mathrm{~A}$ & 0.601622 & 0.813868 & 0.932406 & $0.057 *$ \\
\hline H32B & 0.510293 & 0.764395 & 0.961050 & $0.057^{*}$ \\
\hline $\mathrm{H} 32 \mathrm{C}$ & 0.466450 & 0.852445 & 0.935054 & $0.057^{*}$ \\
\hline $\mathrm{Br} 2$ & $1.50319(4)$ & $0.52837(3)$ & $0.98077(2)$ & $0.04694(11)$ \\
\hline $\mathrm{O} 3$ & $1.1137(2)$ & $0.17437(18)$ & $0.87033(7)$ & $0.0340(5)$ \\
\hline $\mathrm{H} 3 \mathrm{O}$ & 1.149698 & 0.120544 & 0.872362 & $0.051 *$ \\
\hline $\mathrm{O} 4$ & 0.8318 (2) & $0.3046(2)$ & $0.95890(8)$ & $0.0422(6)$ \\
\hline N3 & $1.1594(3)$ & $-0.0081(2)$ & $0.89439(9)$ & $0.0374(7)$ \\
\hline N4 & $0.9864(3)$ & $0.3925(2)$ & $0.98480(8)$ & $0.0365(7)$ \\
\hline $\mathrm{C} 33$ & $0.9444(3)$ & $0.2672(2)$ & $0.89183(9)$ & $0.0295(7)$ \\
\hline H33 & 0.859615 & 0.258280 & 0.900123 & $0.035 *$ \\
\hline C34 & $0.9919(3)$ & $0.1632(2)$ & $0.88238(9)$ & $0.0291(6)$ \\
\hline $\mathrm{C} 35$ & $0.9948(3)$ & $0.0969(2)$ & $0.91712(9)$ & $0.0321(7)$ \\
\hline $\mathrm{H} 35 \mathrm{~A}$ & 1.050174 & 0.125785 & 0.935591 & $0.039 *$ \\
\hline H35B & 0.913825 & 0.095086 & 0.928300 & $0.039 *$ \\
\hline $\mathrm{C} 36$ & $1.0347(3)$ & $-0.0074(3)$ & $0.90853(11)$ & $0.0365(8)$ \\
\hline H36A & 1.029756 & -0.047775 & 0.931298 & $0.044 *$ \\
\hline
\end{tabular}




\begin{tabular}{|c|c|c|c|c|}
\hline H36B & 0.980637 & -0.036456 & 0.889749 & $0.044^{*}$ \\
\hline C37 & $1.2456(4)$ & -0.0081 & $0.92483(14)$ & $0.0522(11)$ \\
\hline H37A & 1.327104 & -0.004144 & 0.914792 & $0.078^{*}$ \\
\hline H37B & 1.237028 & -0.068711 & 0.939203 & $0.078^{*}$ \\
\hline H37C & 1.230662 & 0.048388 & 0.940944 & $0.078^{*}$ \\
\hline C38 & $1.1821(4)$ & $-0.0913(3)$ & $0.86971(13)$ & $0.0510(11)$ \\
\hline H38A & 1.126162 & -0.089017 & 0.848772 & $0.077^{*}$ \\
\hline H38B & 1.170719 & -0.152677 & 0.883403 & $0.077^{*}$ \\
\hline $\mathrm{H} 38 \mathrm{C}$ & 1.264538 & -0.087986 & 0.860431 & $0.077^{*}$ \\
\hline C39 & $0.9167(3)$ & $0.1173(2)$ & $0.85070(10)$ & $0.0331(8)$ \\
\hline $\mathrm{C} 40$ & $0.9708(4)$ & $0.1040(3)$ & $0.81713(11)$ & $0.0480(10)$ \\
\hline $\mathrm{H} 40$ & 1.050579 & 0.126813 & 0.814025 & $0.058^{*}$ \\
\hline C41 & $0.9141(5)$ & $0.0580(4)$ & $0.78685(12)$ & $0.0572(13)$ \\
\hline $\mathrm{H} 41$ & 0.955036 & 0.052016 & 0.763861 & $0.069 *$ \\
\hline $\mathrm{C} 42$ & $0.8021(4)$ & $0.0226(3)$ & $0.79045(11)$ & 0.0489 (11) \\
\hline $\mathrm{H} 42$ & 0.764721 & -0.009785 & 0.770191 & $0.059 *$ \\
\hline $\mathrm{C} 43$ & 0.7401 (4) & $0.0336(3)$ & $0.82435(10)$ & $0.0370(8)$ \\
\hline $\mathrm{C} 44$ & $0.6229(4)$ & $-0.0050(3)$ & $0.82814(12)$ & $0.0438(9)$ \\
\hline $\mathrm{H} 44$ & 0.587964 & -0.039705 & 0.808023 & $0.053 *$ \\
\hline $\mathrm{C} 45$ & $0.5592(4)$ & 0.0068 (3) & $0.86000(13)$ & $0.0468(10)$ \\
\hline $\mathrm{H} 45$ & 0.481437 & -0.020876 & 0.862275 & $0.056^{*}$ \\
\hline $\mathrm{C} 46$ & $0.6092(4)$ & $0.0603(3)$ & $0.88956(11)$ & $0.0389(8)$ \\
\hline $\mathrm{H} 46$ & 0.563583 & 0.070801 & 0.911439 & $0.047 *$ \\
\hline $\mathrm{C} 47$ & $0.7224(3)$ & $0.0971(3)$ & $0.88713(9)$ & $0.0328(7)$ \\
\hline $\mathrm{H} 47$ & 0.754336 & 0.132226 & 0.907619 & $0.039 *$ \\
\hline $\mathrm{C} 48$ & $0.7944(3)$ & $0.0846(2)$ & $0.85485(10)$ & $0.0319(7)$ \\
\hline C49 & $0.9402(3)$ & $0.3399(2)$ & $0.85952(10)$ & $0.0283(7)$ \\
\hline $\mathrm{C} 50$ & $0.8419(3)$ & $0.4007(3)$ & 0.85705 (11) & $0.0366(8)$ \\
\hline $\mathrm{H} 50$ & 0.777022 & 0.391304 & 0.873727 & $0.044 *$ \\
\hline $\mathrm{C} 51$ & $0.8348(4)$ & $0.4754(3)$ & $0.83090(12)$ & $0.0438(9)$ \\
\hline H51 & 0.766337 & 0.516697 & 0.830043 & $0.053 *$ \\
\hline C52 & $0.9274(4)$ & $0.4890(3)$ & $0.80626(12)$ & $0.0442(9)$ \\
\hline H52 & 0.922986 & 0.539544 & 0.788192 & $0.053^{*}$ \\
\hline C53 & $1.0272(4)$ & $0.4288(3)$ & $0.80792(11)$ & $0.0459(10)$ \\
\hline H53 & 1.091198 & 0.438060 & 0.790888 & $0.055^{*}$ \\
\hline C54 & $1.0343(3)$ & $0.3547(3)$ & $0.83440(11)$ & $0.0376(8)$ \\
\hline H54 & 1.103315 & 0.313947 & 0.835444 & $0.045^{*}$ \\
\hline C55 & $1.0098(3)$ & 0.3161 (2) & $0.92410(9)$ & $0.0280(6)$ \\
\hline C56 & $1.1266(3)$ & $0.3448(2)$ & $0.92281(9)$ & $0.0298(7)$ \\
\hline H56 & 1.173876 & 0.328393 & 0.901803 & $0.036^{*}$ \\
\hline C57 & $1.1792(3)$ & $0.3992(3)$ & $0.95242(9)$ & $0.0291(7)$ \\
\hline C58 & 1.2989 (3) & $0.4300(3)$ & $0.95237(10)$ & $0.0315(7)$ \\
\hline $\mathrm{H} 58$ & 1.350752 & 0.411765 & 0.932661 & $0.038^{*}$ \\
\hline C59 & $1.3406(3)$ & $0.4866(3)$ & 0.98087 (11) & $0.0353(7)$ \\
\hline C60 & $1.2659(4)$ & $0.5160(3)$ & $1.01026(10)$ & $0.0410(9)$ \\
\hline $\mathrm{H} 60$ & 1.296029 & 0.556768 & 1.029467 & $0.049 *$ \\
\hline C61 & $1.1491(4)$ & $0.4850(3)$ & $1.01081(10)$ & $0.0414(9)$ \\
\hline H61 & 1.098304 & 0.504605 & 1.030619 & $0.050 *$ \\
\hline
\end{tabular}




$\begin{array}{lllll}\text { C62 } & 1.1029(3) & 0.4246(2) & 0.98254(10) & 0.0320(7) \\ \text { C63 } & 0.9454(3) & 0.3401(3) & 0.95731(10) & 0.0316(7) \\ \text { C64 } & 0.7628(4) & 0.3276(4) & 0.99162(13) & 0.0553(12) \\ \text { H64A } & 0.755306 & 0.398868 & 0.994016 & 0.083^{*} \\ \text { H64B } & 0.803387 & 0.301156 & 1.013550 & 0.083^{*} \\ \text { H64C } & 0.682908 & 0.298312 & 0.989498 & 0.083^{*}\end{array}$

Atomic displacement parameters $\left(\AA^{2}\right)$

\begin{tabular}{|c|c|c|c|c|c|c|}
\hline & $U^{11}$ & $U^{22}$ & $U^{33}$ & $U^{12}$ & $U^{13}$ & $U^{23}$ \\
\hline $\mathrm{Br} 1$ & $0.02814(17)$ & $0.0671(3)$ & $0.0478(2)$ & $-0.00323(18)$ & $0.00642(17)$ & $-0.0063(2)$ \\
\hline $\mathrm{O} 1$ & $0.0292(11)$ & $0.0352(12)$ & $0.0256(12)$ & $-0.0012(10)$ & $-0.0035(9)$ & $-0.0023(10)$ \\
\hline $\mathrm{O} 2$ & $0.0302(13)$ & $0.0367(12)$ & $0.0264(11)$ & $-0.0050(10)$ & $-0.0010(10)$ & $-0.0094(10)$ \\
\hline N1 & $0.0375(18)$ & $0.0485(19)$ & $0.0262(15)$ & $0.0042(14)$ & $-0.0004(12)$ & 0.0038 (14) \\
\hline N2 & $0.0315(15)$ & $0.0329(14)$ & $0.0223(13)$ & $-0.0007(11)$ & $0.0021(11)$ & $-0.0026(12)$ \\
\hline $\mathrm{C} 1$ & $0.0232(14)$ & $0.0247(15)$ & $0.0269(15)$ & $-0.0009(12)$ & $0.0017(11)$ & $-0.0013(13)$ \\
\hline $\mathrm{C} 2$ & $0.0264(15)$ & $0.0260(16)$ & $0.0258(16)$ & $-0.0017(12)$ & $0.0012(12)$ & $-0.0015(13)$ \\
\hline $\mathrm{C} 3$ & $0.0282(16)$ & $0.0296(17)$ & $0.0285(17)$ & $0.0027(13)$ & $0.0001(13)$ & $-0.0026(13)$ \\
\hline $\mathrm{C} 4$ & $0.0321(18)$ & $0.0327(18)$ & 0.0344 (19) & $0.0013(14)$ & $0.0026(14)$ & $0.0046(15)$ \\
\hline $\mathrm{C} 5$ & $0.037(2)$ & $0.078(3)$ & $0.050(3)$ & $0.010(2)$ & $-0.0086(19)$ & $0.005(2)$ \\
\hline $\mathrm{C} 6$ & $0.072(3)$ & $0.062(3)$ & $0.029(2)$ & $0.005(2)$ & $0.002(2)$ & $0.010(2)$ \\
\hline $\mathrm{C} 7$ & $0.0307(17)$ & $0.0271(16)$ & $0.0298(17)$ & $0.0035(12)$ & $0.0046(13)$ & $0.0022(13)$ \\
\hline $\mathrm{C} 8$ & $0.043(2)$ & $0.0351(19)$ & $0.0295(17)$ & $0.0049(15)$ & $0.0079(14)$ & $-0.0011(15)$ \\
\hline C9 & $0.051(2)$ & $0.042(2)$ & $0.037(2)$ & 0.0125 (19) & $0.0171(17)$ & 0.0035 (18) \\
\hline $\mathrm{C} 10$ & $0.046(2)$ & $0.052(2)$ & $0.045(2)$ & $0.013(2)$ & $0.023(2)$ & 0.0110 (19) \\
\hline C11 & $0.033(2)$ & $0.040(2)$ & $0.056(3)$ & $0.0050(15)$ & $0.0146(17)$ & 0.0145 (19) \\
\hline $\mathrm{C} 12$ & $0.033(2)$ & $0.059(3)$ & $0.086(4)$ & $-0.001(2)$ & $0.020(2)$ & $0.020(3)$ \\
\hline $\mathrm{C} 13$ & $0.033(2)$ & $0.059(3)$ & $0.096(4)$ & $-0.010(2)$ & $0.007(2)$ & $0.005(3)$ \\
\hline $\mathrm{C} 14$ & $0.036(2)$ & $0.052(2)$ & $0.071(3)$ & $-0.015(2)$ & $-0.004(2)$ & $0.007(2)$ \\
\hline $\mathrm{C} 15$ & $0.0329(19)$ & $0.0330(19)$ & $0.047(2)$ & $-0.0018(15)$ & $-0.0004(16)$ & $0.0034(17)$ \\
\hline $\mathrm{C} 16$ & $0.0319(18)$ & $0.0290(18)$ & $0.040(2)$ & $0.0031(14)$ & $0.0044(15)$ & $0.0076(15)$ \\
\hline $\mathrm{C} 17$ & $0.0270(15)$ & $0.0264(16)$ & $0.0279(16)$ & $0.0022(13)$ & $0.0045(12)$ & $-0.0035(13)$ \\
\hline $\mathrm{C} 18$ & $0.037(2)$ & $0.0321(17)$ & $0.039(2)$ & $0.0034(14)$ & $0.0005(15)$ & $0.0015(15)$ \\
\hline $\mathrm{C} 19$ & $0.041(2)$ & $0.036(2)$ & $0.059(3)$ & $0.0074(16)$ & $0.0006(18)$ & $0.0106(19)$ \\
\hline $\mathrm{C} 20$ & $0.043(2)$ & $0.0276(18)$ & $0.070(3)$ & $0.0073(16)$ & $0.014(2)$ & $0.0029(19)$ \\
\hline $\mathrm{C} 21$ & $0.043(2)$ & $0.0295(19)$ & $0.064(3)$ & $-0.0002(16)$ & $0.003(2)$ & $-0.0094(18)$ \\
\hline $\mathrm{C} 22$ & $0.038(2)$ & $0.0273(18)$ & $0.047(2)$ & $-0.0003(14)$ & $-0.0023(16)$ & $-0.0066(16)$ \\
\hline $\mathrm{C} 23$ & $0.0311(16)$ & $0.0241(15)$ & $0.0213(15)$ & $0.0004(12)$ & $0.0019(12)$ & $-0.0010(12)$ \\
\hline $\mathrm{C} 24$ & $0.0297(15)$ & $0.0251(15)$ & $0.0251(15)$ & $-0.0003(13)$ & $0.0014(13)$ & $-0.0024(13)$ \\
\hline $\mathrm{C} 25$ & $0.0264(15)$ & $0.0256(16)$ & $0.0265(16)$ & $0.0001(12)$ & $0.0001(12)$ & $0.0025(12)$ \\
\hline $\mathrm{C} 26$ & $0.0284(16)$ & $0.0324(16)$ & $0.0301(16)$ & $0.0025(14)$ & $0.0030(13)$ & $-0.0006(14)$ \\
\hline $\mathrm{C} 27$ & $0.0232(15)$ & $0.0424(19)$ & $0.0342(18)$ & $0.0026(13)$ & $0.0019(14)$ & $0.0022(16)$ \\
\hline $\mathrm{C} 28$ & $0.0317(19)$ & $0.049(2)$ & $0.0299(18)$ & $0.0033(15)$ & $0.0058(14)$ & $-0.0046(15)$ \\
\hline $\mathrm{C} 29$ & $0.0317(18)$ & $0.044(2)$ & $0.0270(17)$ & $0.0024(15)$ & $0.0028(14)$ & $-0.0069(15)$ \\
\hline C30 & $0.0316(16)$ & $0.0300(16)$ & $0.0220(15)$ & $0.0037(12)$ & $0.0000(13)$ & $0.0022(14)$ \\
\hline $\mathrm{C} 31$ & $0.0255(15)$ & $0.0282(16)$ & $0.0242(15)$ & $-0.0005(12)$ & $-0.0035(12)$ & 0.0007 (13) \\
\hline C32 & $0.0321(18)$ & $0.047(2)$ & $0.0349(18)$ & $-0.0078(15)$ & $-0.0054(15)$ & $-0.0130(16)$ \\
\hline $\mathrm{Br} 2$ & $0.0435(2)$ & $0.0550(2)$ & $0.0423(2)$ & $-0.00769(19)$ & $-0.00983(19)$ & $-0.00133(19)$ \\
\hline
\end{tabular}




\begin{tabular}{|c|c|c|c|c|c|c|}
\hline $\mathrm{O} 3$ & $0.0301(12)$ & 0.0305 (12) & $0.0415(14)$ & $0.0025(10)$ & $0.0129(11)$ & $-0.0005(11)$ \\
\hline $\mathrm{O} 4$ & $0.0351(14)$ & $0.0499(16)$ & $0.0415(16)$ & $-0.0055(12)$ & $0.0175(12)$ & -0.0080 \\
\hline N3 & $0.0394(17)$ & $0.0342(17)$ & 0.0387 (17) & $0.0073(13)$ & $0.0039(14)$ & $-0.0059(13)$ \\
\hline N4 & $0.0492(18)$ & $0.0350(15)$ & $0.0253(14)$ & $0.0036(14)$ & $0.0091(13)$ & $0.0005(12)$ \\
\hline C33 & $0.0293(16)$ & $0.0318(17)$ & $0.0274(17)$ & $0.0002(13)$ & $0.0044(13)$ & $-0.0012(13)$ \\
\hline C34 & 0.0295 (16) & $0.0280(15)$ & $0.0298(16)$ & $-0.0017(13)$ & $0.0078(14)$ & $-0.0003(12)$ \\
\hline $\mathrm{C} 35$ & $0.0337(16)$ & $0.0310(16)$ & $0.0316(16)$ & $0.0022(15)$ & $0.0063(14)$ & $0.0028(13)$ \\
\hline $\mathrm{C} 36$ & $0.0364(19)$ & $0.0334(18)$ & $0.040(2)$ & $0.0002(14)$ & $0.0039(15)$ & $0.0014(15)$ \\
\hline $\mathrm{C} 37$ & $0.040(2)$ & $0.058(3)$ & $0.059(3)$ & $0.0083(18)$ & $-0.0011(19)$ & $-0.009(2)$ \\
\hline $\mathrm{C} 38$ & $0.066(3)$ & $0.037(2)$ & $0.049(2)$ & $0.011(2)$ & $0.011(2)$ & $-0.0114(19)$ \\
\hline C39 & $0.043(2)$ & $0.0280(18)$ & $0.0279(17)$ & $-0.0008(14)$ & $0.0087(14)$ & $0.0006(14)$ \\
\hline $\mathrm{C} 40$ & $0.054(3)$ & $0.055(2)$ & $0.036(2)$ & $-0.013(2)$ & $0.0160(18)$ & $-0.0104(19)$ \\
\hline $\mathrm{C} 41$ & 0.075 & $0.066(3)$ & $0.031(2)$ & $-0.017(2)$ & $0.013(2)$ & $-0.014(2)$ \\
\hline $\mathrm{C} 42$ & $0.069(3)$ & $0.047(2)$ & $0.031(2)$ & $-0.009(2)$ & $0.0019(18)$ & $-0.0097(18)$ \\
\hline $\mathrm{C} 43$ & $0.047(2)$ & $0.0289(17)$ & $0.0348(18)$ & $-0.0035(16)$ & $-0.0022(15)$ & $-0.0009(15)$ \\
\hline $\mathrm{C} 44$ & $0.048(2)$ & $0.040(2)$ & $0.044(2)$ & $-0.0053(17)$ & $-0.0108(18)$ & $-0.0002(17)$ \\
\hline $\mathrm{C} 45$ & $0.045(2)$ & $0.042(2)$ & $0.053(3)$ & $-0.0093(17)$ & $-0.009(2)$ & 0.0095 (19) \\
\hline $\mathrm{C} 46$ & 0.0363 (19) & $0.042(2)$ & $0.039(2)$ & $-0.0012(16)$ & $0.0024(16)$ & $0.0106(16)$ \\
\hline $\mathrm{C} 47$ & $0.0379(17)$ & $0.0302(16)$ & $0.0302(17)$ & $-0.0001(16)$ & $0.0023(14)$ & $0.0024(14)$ \\
\hline $\mathrm{C} 48$ & $0.041(2)$ & $0.0246(16)$ & $0.0305(17)$ & $-0.0002(14)$ & $0.0021(14)$ & $0.0027(13)$ \\
\hline $\mathrm{C} 49$ & $0.0282(16)$ & $0.0276(16)$ & $0.0290(17)$ & $-0.0015(13)$ & $0.0010(13)$ & $-0.0028(13)$ \\
\hline $\mathrm{C} 50$ & 0.0339 (18) & 0.0365 (19) & $0.039(2)$ & $0.0036(16)$ & $0.0004(15)$ & $-0.0022(17)$ \\
\hline C51 & $0.045(2)$ & $0.041(2)$ & $0.044(2)$ & $0.0093(18)$ & $-0.0049(17)$ & 0.0024 (19) \\
\hline C52 & $0.051(2)$ & $0.043(2)$ & $0.039(2)$ & $0.0023(18)$ & $-0.0076(18)$ & $0.0110(17)$ \\
\hline C53 & $0.041(2)$ & $0.058(3)$ & $0.039(2)$ & 0.0013 (18) & $0.0032(16)$ & 0.0168 (18) \\
\hline C54 & $0.0289(18)$ & $0.047(2)$ & $0.037(2)$ & $0.0062(15)$ & 0.0027 (14) & 0.0085 (16) \\
\hline C55 & $0.0308(16)$ & $0.0248(14)$ & $0.0283(16)$ & $0.0043(13)$ & $0.0044(13)$ & $0.0014(12)$ \\
\hline C56 & $0.0375(18)$ & $0.0282(17)$ & $0.0236(16)$ & $-0.0006(13)$ & $0.0062(13)$ & $-0.0008(13)$ \\
\hline C57 & $0.0353(17)$ & $0.0286(16)$ & $0.0234(15)$ & $0.0026(14)$ & $0.0032(13)$ & $0.0040(14)$ \\
\hline C58 & $0.0327(18)$ & $0.0343(18)$ & 0.0275 (17) & $0.0014(13)$ & $0.0011(13)$ & 0.0023 (14) \\
\hline C59 & 0.0399 (19) & $0.0352(18)$ & 0.0309 (17) & $-0.0005(14)$ & $-0.0068(15)$ & $0.0049(16)$ \\
\hline C60 & $0.055(2)$ & $0.042(2)$ & $0.0260(17)$ & 0.0029 (19) & $-0.0049(16)$ & $-0.0027(15)$ \\
\hline C61 & $0.061(3)$ & $0.039(2)$ & $0.0234(17)$ & $0.0043(18)$ & 0.0039 (16) & $-0.0042(15)$ \\
\hline C62 & 0.0422 (19) & $0.0289(16)$ & $0.0250(16)$ & $0.0041(14)$ & $0.0035(15)$ & $0.0022(14)$ \\
\hline C63 & $0.0336(17)$ & $0.0299(17)$ & $0.0312(18)$ & $0.0020(14)$ & $0.0080(14)$ & $0.0022(14)$ \\
\hline C64 & $0.047(3)$ & $0.070(3)$ & $0.049(3)$ & $-0.002(2)$ & $0.030(2)$ & $-0.004(2)$ \\
\hline
\end{tabular}

Geometric parameters $\left(\AA,{ }^{\circ}\right)$

\begin{tabular}{llll}
\hline $\mathrm{Br} 1-\mathrm{C} 27$ & $1.903(3)$ & $\mathrm{Br}-\mathrm{C} 59$ & $1.902(4)$ \\
$\mathrm{O} 1-\mathrm{C} 2$ & $1.421(4)$ & $\mathrm{O} 3-\mathrm{C} 34$ & $1.435(4)$ \\
$\mathrm{O} 1-\mathrm{H} 1 \mathrm{O}$ & 0.8400 & $\mathrm{O} 3-\mathrm{H} 3 \mathrm{O}$ & 0.8400 \\
$\mathrm{O} 2-\mathrm{C} 31$ & $1.354(4)$ & $\mathrm{O} 4-\mathrm{C} 63$ & $1.358(4)$ \\
$\mathrm{O} 2-\mathrm{C} 32$ & $1.437(4)$ & $\mathrm{O} 4-\mathrm{C} 64$ & $1.443(4)$ \\
$\mathrm{N} 1-\mathrm{C} 5$ & $1.461(5)$ & $\mathrm{N} 3-\mathrm{C} 37$ & $1.460(6)$ \\
$\mathrm{N} 1-\mathrm{C} 4$ & $1.470(5)$ & $\mathrm{N} 3-\mathrm{C} 38$ & $1.464(5)$ \\
$\mathrm{N} 1-\mathrm{C} 6$ & $1.477(5)$ & $\mathrm{N} 3-\mathrm{C} 36$ & $1.482(5)$ \\
$\mathrm{N} 2-\mathrm{C} 31$ & $1.311(4)$ & $\mathrm{N} 4-\mathrm{C} 63$ & $1.305(5)$
\end{tabular}




\begin{tabular}{|c|c|c|c|}
\hline $\mathrm{N} 2-\mathrm{C} 30$ & $1.372(4)$ & $\mathrm{N} 4-\mathrm{C} 62$ & $1.374(5)$ \\
\hline $\mathrm{C} 1-\mathrm{C} 17$ & $1.526(4)$ & $\mathrm{C} 33-\mathrm{C} 55$ & $1.526(5)$ \\
\hline $\mathrm{C} 1-\mathrm{C} 23$ & $1.531(4)$ & $\mathrm{C} 33-\mathrm{C} 49$ & $1.531(5)$ \\
\hline $\mathrm{C} 1-\mathrm{C} 2$ & $1.560(5)$ & $\mathrm{C} 33-\mathrm{C} 34$ & $1.552(5)$ \\
\hline $\mathrm{C} 1-\mathrm{H} 1$ & 1.0000 & C $33-\mathrm{H} 33$ & 1.0000 \\
\hline $\mathrm{C} 2-\mathrm{C} 3$ & $1.545(5)$ & $\mathrm{C} 34-\mathrm{C} 35$ & $1.546(5)$ \\
\hline $\mathrm{C} 2-\mathrm{C} 7$ & $1.550(5)$ & C34-C39 & $1.550(5)$ \\
\hline $\mathrm{C} 3-\mathrm{C} 4$ & $1.530(5)$ & $\mathrm{C} 35-\mathrm{C} 36$ & $1.524(5)$ \\
\hline $\mathrm{C} 3-\mathrm{H} 3 \mathrm{~A}$ & 0.9900 & $\mathrm{C} 35-\mathrm{H} 35 \mathrm{~A}$ & 0.9900 \\
\hline C $3-\mathrm{H} 3 \mathrm{~B}$ & 0.9900 & $\mathrm{C} 35-\mathrm{H} 35 \mathrm{~B}$ & 0.9900 \\
\hline $\mathrm{C} 4-\mathrm{H} 4 \mathrm{~A}$ & 0.9900 & $\mathrm{C} 36-\mathrm{H} 36 \mathrm{~A}$ & 0.9900 \\
\hline $\mathrm{C} 4-\mathrm{H} 4 \mathrm{~B}$ & 0.9900 & $\mathrm{C} 36-\mathrm{H} 36 \mathrm{~B}$ & 0.9900 \\
\hline $\mathrm{C} 5-\mathrm{H} 5 \mathrm{~A}$ & 0.9800 & C37-H37A & 0.9800 \\
\hline C5-H5B & 0.9800 & C37-H37B & 0.9800 \\
\hline $\mathrm{C} 5-\mathrm{H} 5 \mathrm{C}$ & 0.9800 & $\mathrm{C} 37-\mathrm{H} 37 \mathrm{C}$ & 0.9800 \\
\hline $\mathrm{C} 6-\mathrm{H} 6 \mathrm{~A}$ & 0.9800 & $\mathrm{C} 38-\mathrm{H} 38 \mathrm{~A}$ & 0.9800 \\
\hline C6-H6B & 0.9800 & C $38-\mathrm{H} 38 \mathrm{~B}$ & 0.9800 \\
\hline C6- $\mathrm{H} 6 \mathrm{C}$ & 0.9800 & $\mathrm{C} 38-\mathrm{H} 38 \mathrm{C}$ & 0.9800 \\
\hline $\mathrm{C} 7-\mathrm{C} 8$ & $1.377(5)$ & $\mathrm{C} 39-\mathrm{C} 40$ & $1.365(5)$ \\
\hline $\mathrm{C} 7-\mathrm{C} 16$ & $1.449(5)$ & $\mathrm{C} 39-\mathrm{C} 48$ & $1.443(5)$ \\
\hline $\mathrm{C} 8-\mathrm{C} 9$ & $1.417(5)$ & $\mathrm{C} 40-\mathrm{C} 41$ & $1.409(6)$ \\
\hline $\mathrm{C} 8-\mathrm{H} 8$ & 0.9500 & $\mathrm{C} 40-\mathrm{H} 40$ & 0.9500 \\
\hline $\mathrm{C} 9-\mathrm{C} 10$ & $1.346(7)$ & $\mathrm{C} 41-\mathrm{C} 42$ & $1.347(7)$ \\
\hline C9- & 0.9500 & C41-H41 & 0.9500 \\
\hline $\mathrm{C} 10-\mathrm{C} 11$ & $1.413(6)$ & $\mathrm{C} 42-\mathrm{C} 43$ & $1.412(6)$ \\
\hline $\mathrm{C} 10-\mathrm{H} 10$ & 0.9500 & $\mathrm{C} 42-\mathrm{H} 42$ & 0.9500 \\
\hline $\mathrm{C} 11-\mathrm{C} 12$ & $1.404(7)$ & $\mathrm{C} 43-\mathrm{C} 44$ & $1.416(6)$ \\
\hline $\mathrm{C} 11-\mathrm{C} 16$ & $1.438(5)$ & $\mathrm{C} 43-\mathrm{C} 48$ & $1.436(5)$ \\
\hline $\mathrm{C} 12-\mathrm{C} 13$ & $1.366(8)$ & $\mathrm{C} 44-\mathrm{C} 45$ & $1.360(6)$ \\
\hline $\mathrm{C} 12-\mathrm{H} 12$ & 0.9500 & $\mathrm{C} 44-\mathrm{H} 44$ & 0.9500 \\
\hline $\mathrm{C} 13-\mathrm{C} 14$ & $1.384(7)$ & $\mathrm{C} 45-\mathrm{C} 46$ & $1.407(6)$ \\
\hline C13-H13 & 0.9500 & $\mathrm{C} 45-\mathrm{H} 45$ & 0.9500 \\
\hline $\mathrm{C} 14-\mathrm{C} 15$ & $1.371(6)$ & $\mathrm{C} 46-\mathrm{C} 47$ & $1.362(5)$ \\
\hline C14-H14 & 0.9500 & $\mathrm{C} 46-\mathrm{H} 46$ & 0.9500 \\
\hline $\mathrm{C} 15-\mathrm{C} 16$ & $1.420(6)$ & $\mathrm{C} 47-\mathrm{C} 48$ & $1.425(5)$ \\
\hline C15-H15 & 0.9500 & $\mathrm{C} 47-\mathrm{H} 47$ & 0.9500 \\
\hline $\mathrm{C} 17-\mathrm{C} 22$ & $1.391(5)$ & $\mathrm{C} 49-\mathrm{C} 50$ & $1.378(5)$ \\
\hline $\mathrm{C} 17-\mathrm{C} 18$ & $1.395(5)$ & $\mathrm{C} 49-\mathrm{C} 54$ & $1.402(5)$ \\
\hline $\mathrm{C} 18-\mathrm{C} 19$ & $1.388(5)$ & $\mathrm{C} 50-\mathrm{C} 51$ & $1.390(6)$ \\
\hline C18-H18 & 0.9500 & $\mathrm{C} 50-\mathrm{H} 50$ & 0.9500 \\
\hline $\mathrm{C} 19-\mathrm{C} 20$ & $1.382(6)$ & $\mathrm{C} 51-\mathrm{C} 52$ & $1.376(6)$ \\
\hline C19-H19 & 0.9500 & C51-H51 & 0.9500 \\
\hline $\mathrm{C} 20-\mathrm{C} 21$ & $1.372(6)$ & $\mathrm{C} 52-\mathrm{C} 53$ & $1.385(6)$ \\
\hline $\mathrm{C} 20-\mathrm{H} 20$ & 0.9500 & C52-H52 & 0.9500 \\
\hline $\mathrm{C} 21-\mathrm{C} 22$ & $1.385(5)$ & $\mathrm{C} 53-\mathrm{C} 54$ & $1.393(5)$ \\
\hline $\mathrm{C} 21-\mathrm{H} 21$ & 0.9500 & C53-H53 & 0.9500 \\
\hline $\mathrm{C} 22-\mathrm{H} 22$ & 0.9500 & C54-H54 & 0.9500 \\
\hline $\mathrm{C} 23-\mathrm{C} 24$ & $1.361(5)$ & C55-C56 & $1.362(5)$ \\
\hline
\end{tabular}




\begin{tabular}{|c|c|c|c|}
\hline $\mathrm{C} 23-\mathrm{C} 31$ & $1.435(4)$ & $\mathrm{C} 55-\mathrm{C} 63$ & $1.435(5)$ \\
\hline $\mathrm{C} 24-\mathrm{C} 25$ & $1.424(4)$ & $\mathrm{C} 56-\mathrm{C} 57$ & $1.426(5)$ \\
\hline $\mathrm{C} 24-\mathrm{H} 24$ & 0.9500 & C56-H56 & 0.9500 \\
\hline $\mathrm{C} 25-\mathrm{C} 26$ & $1.416(5)$ & $\mathrm{C} 57-\mathrm{C} 58$ & $1.400(5)$ \\
\hline $\mathrm{C} 25-\mathrm{C} 30$ & $1.421(5)$ & $\mathrm{C} 57-\mathrm{C} 62$ & $1.423(5)$ \\
\hline $\mathrm{C} 26-\mathrm{C} 27$ & $1.365(5)$ & $\mathrm{C} 58-\mathrm{C} 59$ & $1.367(5)$ \\
\hline $\mathrm{C} 26-\mathrm{H} 26$ & 0.9500 & C58-H58 & 0.9500 \\
\hline $\mathrm{C} 27-\mathrm{C} 28$ & $1.409(5)$ & $\mathrm{C} 59-\mathrm{C} 60$ & $1.406(6)$ \\
\hline $\mathrm{C} 28-\mathrm{C} 29$ & $1.369(5)$ & $\mathrm{C} 60-\mathrm{C} 61$ & $1.371(6)$ \\
\hline $\mathrm{C} 28-\mathrm{H} 28$ & 0.9500 & $\mathrm{C} 60-\mathrm{H} 60$ & 0.9500 \\
\hline $\mathrm{C} 29-\mathrm{C} 30$ & $1.418(5)$ & C61-C62 & $1.409(5)$ \\
\hline $\mathrm{C} 29-\mathrm{H} 29$ & 0.9500 & C61-H61 & 0.9500 \\
\hline $\mathrm{C} 32-\mathrm{H} 32 \mathrm{~A}$ & 0.9800 & C64-H64A & 0.9800 \\
\hline C $32-\mathrm{H} 32 \mathrm{~B}$ & 0.9800 & C64-H64B & 0.9800 \\
\hline $\mathrm{C} 32-\mathrm{H} 32 \mathrm{C}$ & 0.9800 & $\mathrm{C} 64-\mathrm{H} 64 \mathrm{C}$ & 0.9800 \\
\hline $\mathrm{C} 2-\mathrm{O} 1-\mathrm{H} 1 \mathrm{O}$ & 109.5 & $\mathrm{C} 34-\mathrm{O} 3-\mathrm{H} 3 \mathrm{O}$ & 109.5 \\
\hline $\mathrm{C} 31-\mathrm{O} 2-\mathrm{C} 32$ & $117.4(3)$ & $\mathrm{C} 63-\mathrm{O} 4-\mathrm{C} 64$ & $117.1(3)$ \\
\hline $\mathrm{C} 5-\mathrm{N} 1-\mathrm{C} 4$ & $111.0(3)$ & $\mathrm{C} 37-\mathrm{N} 3-\mathrm{C} 38$ & $110.1(3)$ \\
\hline $\mathrm{C} 5-\mathrm{N} 1-\mathrm{C} 6$ & $110.1(4)$ & $\mathrm{C} 37-\mathrm{N} 3-\mathrm{C} 36$ & $111.1(3)$ \\
\hline $\mathrm{C} 4-\mathrm{N} 1-\mathrm{C} 6$ & $111.0(3)$ & $\mathrm{C} 38-\mathrm{N} 3-\mathrm{C} 36$ & $112.1(3)$ \\
\hline $\mathrm{C} 31-\mathrm{N} 2-\mathrm{C} 30$ & $117.1(3)$ & $\mathrm{C} 63-\mathrm{N} 4-\mathrm{C} 62$ & $117.5(3)$ \\
\hline $\mathrm{C} 17-\mathrm{C} 1-\mathrm{C} 23$ & $109.9(3)$ & $\mathrm{C} 55-\mathrm{C} 33-\mathrm{C} 49$ & $108.2(3)$ \\
\hline $\mathrm{C} 17-\mathrm{C} 1-\mathrm{C} 2$ & $116.8(3)$ & $\mathrm{C} 55-\mathrm{C} 33-\mathrm{C} 34$ & $113.8(3)$ \\
\hline $\mathrm{C} 23-\mathrm{C} 1-\mathrm{C} 2$ & $112.2(3)$ & $\mathrm{C} 49-\mathrm{C} 33-\mathrm{C} 34$ & $115.8(3)$ \\
\hline $\mathrm{C} 17-\mathrm{C} 1-\mathrm{H} 1$ & 105.7 & $\mathrm{C} 55-\mathrm{C} 33-\mathrm{H} 33$ & 106.1 \\
\hline $\mathrm{C} 23-\mathrm{C} 1-\mathrm{H} 1$ & 105.7 & $\mathrm{C} 49-\mathrm{C} 33-\mathrm{H} 33$ & 106.1 \\
\hline $\mathrm{C} 2-\mathrm{C} 1-\mathrm{H} 1$ & 105.7 & $\mathrm{C} 34-\mathrm{C} 33-\mathrm{H} 33$ & 106.1 \\
\hline $\mathrm{O} 1-\mathrm{C} 2-\mathrm{C} 3$ & $106.7(3)$ & $\mathrm{O} 3-\mathrm{C} 34-\mathrm{C} 35$ & $106.7(3)$ \\
\hline $\mathrm{O} 1-\mathrm{C} 2-\mathrm{C} 7$ & $110.2(3)$ & $\mathrm{O} 3-\mathrm{C} 34-\mathrm{C} 39$ & $109.5(3)$ \\
\hline $\mathrm{C} 3-\mathrm{C} 2-\mathrm{C} 7$ & $109.0(3)$ & $\mathrm{C} 35-\mathrm{C} 34-\mathrm{C} 39$ & $111.8(3)$ \\
\hline $\mathrm{O} 1-\mathrm{C} 2-\mathrm{C} 1$ & $107.1(3)$ & $\mathrm{O} 3-\mathrm{C} 34-\mathrm{C} 33$ & $107.0(3)$ \\
\hline $\mathrm{C} 3-\mathrm{C} 2-\mathrm{C} 1$ & $112.1(3)$ & $\mathrm{C} 35-\mathrm{C} 34-\mathrm{C} 33$ & $111.3(3)$ \\
\hline $\mathrm{C} 7-\mathrm{C} 2-\mathrm{C} 1$ & $111.7(3)$ & $\mathrm{C} 39-\mathrm{C} 34-\mathrm{C} 33$ & $110.3(3)$ \\
\hline $\mathrm{C} 4-\mathrm{C} 3-\mathrm{C} 2$ & $112.8(3)$ & $\mathrm{C} 36-\mathrm{C} 35-\mathrm{C} 34$ & $112.8(3)$ \\
\hline $\mathrm{C} 4-\mathrm{C} 3-\mathrm{H} 3 \mathrm{~A}$ & 109.0 & $\mathrm{C} 36-\mathrm{C} 35-\mathrm{H} 35 \mathrm{~A}$ & 109.0 \\
\hline $\mathrm{C} 2-\mathrm{C} 3-\mathrm{H} 3 \mathrm{~A}$ & 109.0 & $\mathrm{C} 34-\mathrm{C} 35-\mathrm{H} 35 \mathrm{~A}$ & 109.0 \\
\hline $\mathrm{C} 4-\mathrm{C} 3-\mathrm{H} 3 \mathrm{~B}$ & 109.0 & $\mathrm{C} 36-\mathrm{C} 35-\mathrm{H} 35 \mathrm{~B}$ & 109.0 \\
\hline $\mathrm{C} 2-\mathrm{C} 3-\mathrm{H} 3 \mathrm{~B}$ & 109.0 & $\mathrm{C} 34-\mathrm{C} 35-\mathrm{H} 35 \mathrm{~B}$ & 109.0 \\
\hline $\mathrm{H} 3 \mathrm{~A}-\mathrm{C} 3-\mathrm{H} 3 \mathrm{~B}$ & 107.8 & $\mathrm{H} 35 \mathrm{~A}-\mathrm{C} 35-\mathrm{H} 35 \mathrm{~B}$ & 107.8 \\
\hline $\mathrm{N} 1-\mathrm{C} 4-\mathrm{C} 3$ & $112.2(3)$ & $\mathrm{N} 3-\mathrm{C} 36-\mathrm{C} 35$ & $110.5(3)$ \\
\hline $\mathrm{N} 1-\mathrm{C} 4-\mathrm{H} 4 \mathrm{~A}$ & 109.2 & $\mathrm{~N} 3-\mathrm{C} 36-\mathrm{H} 36 \mathrm{~A}$ & 109.5 \\
\hline $\mathrm{C} 3-\mathrm{C} 4-\mathrm{H} 4 \mathrm{~A}$ & 109.2 & $\mathrm{C} 35-\mathrm{C} 36-\mathrm{H} 36 \mathrm{~A}$ & 109.5 \\
\hline $\mathrm{N} 1-\mathrm{C} 4-\mathrm{H} 4 \mathrm{~B}$ & 109.2 & N3-C $36-\mathrm{H} 36 \mathrm{~B}$ & 109.5 \\
\hline $\mathrm{C} 3-\mathrm{C} 4-\mathrm{H} 4 \mathrm{~B}$ & 109.2 & $\mathrm{C} 35-\mathrm{C} 36-\mathrm{H} 36 \mathrm{~B}$ & 109.5 \\
\hline $\mathrm{H} 4 \mathrm{~A}-\mathrm{C} 4-\mathrm{H} 4 \mathrm{~B}$ & 107.9 & $\mathrm{H} 36 \mathrm{~A}-\mathrm{C} 36-\mathrm{H} 36 \mathrm{~B}$ & 108.1 \\
\hline $\mathrm{N} 1-\mathrm{C} 5-\mathrm{H} 5 \mathrm{~A}$ & 109.5 & $\mathrm{~N} 3-\mathrm{C} 37-\mathrm{H} 37 \mathrm{~A}$ & 109.5 \\
\hline $\mathrm{N} 1-\mathrm{C} 5-\mathrm{H} 5 \mathrm{~B}$ & 109.5 & $\mathrm{~N} 3-\mathrm{C} 37-\mathrm{H} 37 \mathrm{~B}$ & 109.5 \\
\hline
\end{tabular}




\begin{tabular}{|c|c|}
\hline $\mathrm{H} 5 \mathrm{~A}-\mathrm{C} 5-\mathrm{H} 5 \mathrm{~B}$ & 109.5 \\
\hline $\mathrm{N} 1-\mathrm{C} 5-\mathrm{H} 5 \mathrm{C}$ & 109.5 \\
\hline $\mathrm{H} 5 \mathrm{~A}-\mathrm{C} 5-\mathrm{H} 5 \mathrm{C}$ & 109.5 \\
\hline $\mathrm{H} 5 \mathrm{~B}-\mathrm{C} 5-\mathrm{H} 5 \mathrm{C}$ & 109.5 \\
\hline $\mathrm{N} 1-\mathrm{C} 6-\mathrm{H} 6 \mathrm{~A}$ & 109.5 \\
\hline $\mathrm{N} 1-\mathrm{C} 6-\mathrm{H} 6 \mathrm{~B}$ & 109.5 \\
\hline $\mathrm{H} 6 \mathrm{~A}-\mathrm{C} 6-\mathrm{H} 6 \mathrm{~B}$ & 109.5 \\
\hline $\mathrm{N} 1-\mathrm{C} 6-\mathrm{H} 6 \mathrm{C}$ & 109.5 \\
\hline $\mathrm{H} 6 \mathrm{~A}-\mathrm{C} 6-\mathrm{H} 6 \mathrm{C}$ & 109.5 \\
\hline $\mathrm{H} 6 \mathrm{~B}-\mathrm{C} 6-\mathrm{H} 6 \mathrm{C}$ & 109.5 \\
\hline $\mathrm{C} 8-\mathrm{C} 7-\mathrm{C} 16$ & $118.4(3)$ \\
\hline $\mathrm{C} 8-\mathrm{C} 7-\mathrm{C} 2$ & $118.0(3)$ \\
\hline $\mathrm{C} 16-\mathrm{C} 7-\mathrm{C} 2$ & $123.2(3)$ \\
\hline $\mathrm{C} 7-\mathrm{C} 8-\mathrm{C} 9$ & $122.2(4)$ \\
\hline $\mathrm{C} 7-\mathrm{C} 8-\mathrm{H} 8$ & 118.9 \\
\hline $\mathrm{C} 9-\mathrm{C} 8-\mathrm{H} 8$ & 118.9 \\
\hline $\mathrm{C} 10-\mathrm{C} 9-\mathrm{C} 8$ & $120.2(4)$ \\
\hline $\mathrm{C} 10-\mathrm{C} 9-\mathrm{H} 9$ & 119.9 \\
\hline $\mathrm{C} 8-\mathrm{C} 9-\mathrm{H} 9$ & 119.9 \\
\hline $\mathrm{C} 9-\mathrm{C} 10-\mathrm{C} 11$ & 120.9 (4) \\
\hline $\mathrm{C} 9-\mathrm{C} 10-\mathrm{H} 10$ & 119.5 \\
\hline $\mathrm{C} 11-\mathrm{C} 10-\mathrm{H} 10$ & 119.5 \\
\hline $\mathrm{C} 12-\mathrm{C} 11-\mathrm{C} 10$ & $120.4(4)$ \\
\hline $\mathrm{C} 12-\mathrm{C} 11-\mathrm{C} 16$ & $119.6(4)$ \\
\hline $\mathrm{C} 10-\mathrm{C} 11-\mathrm{C} 16$ & $119.9(4)$ \\
\hline $\mathrm{C} 13-\mathrm{C} 12-\mathrm{C} 11$ & $121.3(4)$ \\
\hline $\mathrm{C} 13-\mathrm{C} 12-\mathrm{H} 12$ & 119.4 \\
\hline $\mathrm{C} 11-\mathrm{C} 12-\mathrm{H} 12$ & 119.4 \\
\hline $\mathrm{C} 12-\mathrm{C} 13-\mathrm{C} 14$ & $120.0(4)$ \\
\hline $\mathrm{C} 12-\mathrm{C} 13-\mathrm{H} 13$ & 120.0 \\
\hline $\mathrm{C} 14-\mathrm{C} 13-\mathrm{H} 13$ & 120.0 \\
\hline $\mathrm{C} 15-\mathrm{C} 14-\mathrm{C} 13$ & $120.7(5)$ \\
\hline $\mathrm{C} 15-\mathrm{C} 14-\mathrm{H} 14$ & 119.7 \\
\hline $\mathrm{C} 13-\mathrm{C} 14-\mathrm{H} 14$ & 119.7 \\
\hline $\mathrm{C} 14-\mathrm{C} 15-\mathrm{C} 16$ & $121.8(4)$ \\
\hline $\mathrm{C} 14-\mathrm{C} 15-\mathrm{H} 15$ & 119.1 \\
\hline $\mathrm{C} 16-\mathrm{C} 15-\mathrm{H} 15$ & 119.1 \\
\hline $\mathrm{C} 15-\mathrm{C} 16-\mathrm{C} 11$ & $116.5(4)$ \\
\hline $\mathrm{C} 15-\mathrm{C} 16-\mathrm{C} 7$ & $125.3(3)$ \\
\hline $\mathrm{C} 11-\mathrm{C} 16-\mathrm{C} 7$ & $118.2(4)$ \\
\hline $\mathrm{C} 22-\mathrm{C} 17-\mathrm{C} 18$ & $117.3(3)$ \\
\hline $\mathrm{C} 22-\mathrm{C} 17-\mathrm{C} 1$ & $125.2(3)$ \\
\hline $\mathrm{C} 18-\mathrm{C} 17-\mathrm{C} 1$ & $117.3(3)$ \\
\hline $\mathrm{C} 19-\mathrm{C} 18-\mathrm{C} 17$ & $121.3(4)$ \\
\hline $\mathrm{C} 19-\mathrm{C} 18-\mathrm{H} 18$ & 119.4 \\
\hline $\mathrm{C} 17-\mathrm{C} 18-\mathrm{H} 18$ & 119.4 \\
\hline $\mathrm{C} 20-\mathrm{C} 19-\mathrm{C} 18$ & $120.5(4)$ \\
\hline $\mathrm{C} 20-\mathrm{C} 19-\mathrm{H} 19$ & 119.7 \\
\hline
\end{tabular}

\begin{tabular}{|c|c|}
\hline $\mathrm{H} 37 \mathrm{~A}-\mathrm{C} 37-\mathrm{H} 37 \mathrm{~B}$ & 109.5 \\
\hline N3-C37-H37C & 109.5 \\
\hline $\mathrm{H} 37 \mathrm{~A}-\mathrm{C} 37-\mathrm{H} 37 \mathrm{C}$ & 109.5 \\
\hline $\mathrm{H} 37 \mathrm{~B}-\mathrm{C} 37-\mathrm{H} 37 \mathrm{C}$ & 109.5 \\
\hline $\mathrm{N} 3-\mathrm{C} 38-\mathrm{H} 38 \mathrm{~A}$ & 109.5 \\
\hline N3-C38-H38B & 109.5 \\
\hline $\mathrm{H} 38 \mathrm{~A}-\mathrm{C} 38-\mathrm{H} 38 \mathrm{~B}$ & 109.5 \\
\hline $\mathrm{N} 3-\mathrm{C} 38-\mathrm{H} 38 \mathrm{C}$ & 109.5 \\
\hline $\mathrm{H} 38 \mathrm{~A}-\mathrm{C} 38-\mathrm{H} 38 \mathrm{C}$ & 109.5 \\
\hline $\mathrm{H} 38 \mathrm{~B}-\mathrm{C} 38-\mathrm{H} 38 \mathrm{C}$ & 109.5 \\
\hline $\mathrm{C} 40-\mathrm{C} 39-\mathrm{C} 48$ & $118.0(4)$ \\
\hline $\mathrm{C} 40-\mathrm{C} 39-\mathrm{C} 34$ & $117.9(3)$ \\
\hline $\mathrm{C} 48-\mathrm{C} 39-\mathrm{C} 34$ & $124.1(3)$ \\
\hline $\mathrm{C} 39-\mathrm{C} 40-\mathrm{C} 41$ & $123.2(4)$ \\
\hline $\mathrm{C} 39-\mathrm{C} 40-\mathrm{H} 40$ & 118.4 \\
\hline $\mathrm{C} 41-\mathrm{C} 40-\mathrm{H} 40$ & 118.4 \\
\hline $\mathrm{C} 42-\mathrm{C} 41-\mathrm{C} 40$ & $120.1(4)$ \\
\hline $\mathrm{C} 42-\mathrm{C} 41-\mathrm{H} 41$ & 119.9 \\
\hline $\mathrm{C} 40-\mathrm{C} 41-\mathrm{H} 41$ & 119.9 \\
\hline $\mathrm{C} 41-\mathrm{C} 42-\mathrm{C} 43$ & $120.0(4)$ \\
\hline $\mathrm{C} 41-\mathrm{C} 42-\mathrm{H} 42$ & 120.0 \\
\hline $\mathrm{C} 43-\mathrm{C} 42-\mathrm{H} 42$ & 120.0 \\
\hline $\mathrm{C} 42-\mathrm{C} 43-\mathrm{C} 44$ & $119.8(4)$ \\
\hline $\mathrm{C} 42-\mathrm{C} 43-\mathrm{C} 48$ & $120.5(4)$ \\
\hline $\mathrm{C} 44-\mathrm{C} 43-\mathrm{C} 48$ & $119.7(4)$ \\
\hline $\mathrm{C} 45-\mathrm{C} 44-\mathrm{C} 43$ & $121.3(4)$ \\
\hline $\mathrm{C} 45-\mathrm{C} 44-\mathrm{H} 44$ & 119.3 \\
\hline $\mathrm{C} 43-\mathrm{C} 44-\mathrm{H} 44$ & 119.3 \\
\hline $\mathrm{C} 44-\mathrm{C} 45-\mathrm{C} 46$ & $119.6(4)$ \\
\hline $\mathrm{C} 44-\mathrm{C} 45-\mathrm{H} 45$ & 120.2 \\
\hline $\mathrm{C} 46-\mathrm{C} 45-\mathrm{H} 45$ & 120.2 \\
\hline $\mathrm{C} 47-\mathrm{C} 46-\mathrm{C} 45$ & $120.7(4)$ \\
\hline $\mathrm{C} 47-\mathrm{C} 46-\mathrm{H} 46$ & 119.7 \\
\hline $\mathrm{C} 45-\mathrm{C} 46-\mathrm{H} 46$ & 119.7 \\
\hline $\mathrm{C} 46-\mathrm{C} 47-\mathrm{C} 48$ & $122.1(4)$ \\
\hline $\mathrm{C} 46-\mathrm{C} 47-\mathrm{H} 47$ & 119.0 \\
\hline $\mathrm{C} 48-\mathrm{C} 47-\mathrm{H} 47$ & 119.0 \\
\hline $\mathrm{C} 47-\mathrm{C} 48-\mathrm{C} 43$ & $116.5(3)$ \\
\hline $\mathrm{C} 47-\mathrm{C} 48-\mathrm{C} 39$ & $125.5(3)$ \\
\hline $\mathrm{C} 43-\mathrm{C} 48-\mathrm{C} 39$ & $118.0(3)$ \\
\hline $\mathrm{C} 50-\mathrm{C} 49-\mathrm{C} 54$ & $117.9(3)$ \\
\hline $\mathrm{C} 50-\mathrm{C} 49-\mathrm{C} 33$ & $117.6(3)$ \\
\hline $\mathrm{C} 54-\mathrm{C} 49-\mathrm{C} 33$ & $124.2(3)$ \\
\hline $\mathrm{C} 49-\mathrm{C} 50-\mathrm{C} 51$ & $122.0(4)$ \\
\hline $\mathrm{C} 49-\mathrm{C} 50-\mathrm{H} 50$ & 119.0 \\
\hline $\mathrm{C} 51-\mathrm{C} 50-\mathrm{H} 50$ & 119.0 \\
\hline $\mathrm{C} 52-\mathrm{C} 51-\mathrm{C} 50$ & $119.6(4)$ \\
\hline $\mathrm{C} 52-\mathrm{C} 51-\mathrm{H} 51$ & 120.2 \\
\hline
\end{tabular}




\begin{tabular}{|c|c|c|c|}
\hline $\mathrm{C} 18-\mathrm{C} 19-\mathrm{H} 19$ & 119.7 & $\mathrm{C} 50-\mathrm{C} 51-\mathrm{H} 51$ & 120.2 \\
\hline $\mathrm{C} 21-\mathrm{C} 20-\mathrm{C} 19$ & $118.5(4)$ & $\mathrm{C} 51-\mathrm{C} 52-\mathrm{C} 53$ & $119.7(4)$ \\
\hline $\mathrm{C} 21-\mathrm{C} 20-\mathrm{H} 20$ & 120.7 & $\mathrm{C} 51-\mathrm{C} 52-\mathrm{H} 52$ & 120.1 \\
\hline $\mathrm{C} 19-\mathrm{C} 20-\mathrm{H} 20$ & 120.7 & $\mathrm{C} 53-\mathrm{C} 52-\mathrm{H} 52$ & 120.1 \\
\hline $\mathrm{C} 20-\mathrm{C} 21-\mathrm{C} 22$ & $121.4(4)$ & $\mathrm{C} 52-\mathrm{C} 53-\mathrm{C} 54$ & $120.4(4)$ \\
\hline $\mathrm{C} 20-\mathrm{C} 21-\mathrm{H} 21$ & 119.3 & $\mathrm{C} 52-\mathrm{C} 53-\mathrm{H} 53$ & 119.8 \\
\hline $\mathrm{C} 22-\mathrm{C} 21-\mathrm{H} 21$ & 119.3 & $\mathrm{C} 54-\mathrm{C} 53-\mathrm{H} 53$ & 119.8 \\
\hline $\mathrm{C} 21-\mathrm{C} 22-\mathrm{C} 17$ & $120.9(4)$ & $\mathrm{C} 53-\mathrm{C} 54-\mathrm{C} 49$ & $120.3(3)$ \\
\hline $\mathrm{C} 21-\mathrm{C} 22-\mathrm{H} 22$ & 119.5 & $\mathrm{C} 53-\mathrm{C} 54-\mathrm{H} 54$ & 119.8 \\
\hline $\mathrm{C} 17-\mathrm{C} 22-\mathrm{H} 22$ & 119.5 & $\mathrm{C} 49-\mathrm{C} 54-\mathrm{H} 54$ & 119.8 \\
\hline $\mathrm{C} 24-\mathrm{C} 23-\mathrm{C} 31$ & $116.5(3)$ & $\mathrm{C} 56-\mathrm{C} 55-\mathrm{C} 63$ & $116.2(3)$ \\
\hline $\mathrm{C} 24-\mathrm{C} 23-\mathrm{C} 1$ & $124.3(3)$ & $\mathrm{C} 56-\mathrm{C} 55-\mathrm{C} 33$ & $123.8(3)$ \\
\hline $\mathrm{C} 31-\mathrm{C} 23-\mathrm{C} 1$ & $119.2(3)$ & $\mathrm{C} 63-\mathrm{C} 55-\mathrm{C} 33$ & $119.8(3)$ \\
\hline $\mathrm{C} 23-\mathrm{C} 24-\mathrm{C} 25$ & $120.8(3)$ & $\mathrm{C} 55-\mathrm{C} 56-\mathrm{C} 57$ & $121.2(3)$ \\
\hline $\mathrm{C} 23-\mathrm{C} 24-\mathrm{H} 24$ & 119.6 & $\mathrm{C} 55-\mathrm{C} 56-\mathrm{H} 56$ & 119.4 \\
\hline $\mathrm{C} 25-\mathrm{C} 24-\mathrm{H} 24$ & 119.6 & C57-C56-H56 & 119.4 \\
\hline $\mathrm{C} 26-\mathrm{C} 25-\mathrm{C} 30$ & $119.6(3)$ & $\mathrm{C} 58-\mathrm{C} 57-\mathrm{C} 62$ & $119.9(3)$ \\
\hline $\mathrm{C} 26-\mathrm{C} 25-\mathrm{C} 24$ & $123.1(3)$ & $\mathrm{C} 58-\mathrm{C} 57-\mathrm{C} 56$ & $123.2(3)$ \\
\hline $\mathrm{C} 30-\mathrm{C} 25-\mathrm{C} 24$ & $117.3(3)$ & $\mathrm{C} 62-\mathrm{C} 57-\mathrm{C} 56$ & $116.9(3)$ \\
\hline $\mathrm{C} 27-\mathrm{C} 26-\mathrm{C} 25$ & $119.2(3)$ & $\mathrm{C} 59-\mathrm{C} 58-\mathrm{C} 57$ & $119.6(3)$ \\
\hline $\mathrm{C} 27-\mathrm{C} 26-\mathrm{H} 26$ & 120.4 & $\mathrm{C} 59-\mathrm{C} 58-\mathrm{H} 58$ & 120.2 \\
\hline $\mathrm{C} 25-\mathrm{C} 26-\mathrm{H} 26$ & 120.4 & $\mathrm{C} 57-\mathrm{C} 58-\mathrm{H} 58$ & 120.2 \\
\hline $\mathrm{C} 26-\mathrm{C} 27-\mathrm{C} 28$ & $122.2(3)$ & $\mathrm{C} 58-\mathrm{C} 59-\mathrm{C} 60$ & $121.7(4)$ \\
\hline $\mathrm{C} 26-\mathrm{C} 27-\mathrm{Br} 1$ & $120.1(3)$ & $\mathrm{C} 58-\mathrm{C} 59-\mathrm{Br} 2$ & $119.5(3)$ \\
\hline $\mathrm{C} 28-\mathrm{C} 27-\mathrm{Br} 1$ & $117.7(3)$ & $\mathrm{C} 60-\mathrm{C} 59-\mathrm{Br} 2$ & $118.8(3)$ \\
\hline $\mathrm{C} 29-\mathrm{C} 28-\mathrm{C} 27$ & $119.3(3)$ & $\mathrm{C} 61-\mathrm{C} 60-\mathrm{C} 59$ & $119.1(4)$ \\
\hline $\mathrm{C} 29-\mathrm{C} 28-\mathrm{H} 28$ & 120.4 & $\mathrm{C} 61-\mathrm{C} 60-\mathrm{H} 60$ & 120.4 \\
\hline $\mathrm{C} 27-\mathrm{C} 28-\mathrm{H} 28$ & 120.4 & $\mathrm{C} 59-\mathrm{C} 60-\mathrm{H} 60$ & 120.4 \\
\hline $\mathrm{C} 28-\mathrm{C} 29-\mathrm{C} 30$ & $120.8(3)$ & $\mathrm{C} 60-\mathrm{C} 61-\mathrm{C} 62$ & $121.2(4)$ \\
\hline $\mathrm{C} 28-\mathrm{C} 29-\mathrm{H} 29$ & 119.6 & $\mathrm{C} 60-\mathrm{C} 61-\mathrm{H} 61$ & 119.4 \\
\hline $\mathrm{C} 30-\mathrm{C} 29-\mathrm{H} 29$ & 119.6 & C62-C61-H61 & 119.4 \\
\hline $\mathrm{N} 2-\mathrm{C} 30-\mathrm{C} 29$ & $118.5(3)$ & N4-C62-C61 & $119.3(3)$ \\
\hline $\mathrm{N} 2-\mathrm{C} 30-\mathrm{C} 25$ & $122.5(3)$ & N4-C62-C57 & $122.3(3)$ \\
\hline $\mathrm{C} 29-\mathrm{C} 30-\mathrm{C} 25$ & $118.9(3)$ & $\mathrm{C} 61-\mathrm{C} 62-\mathrm{C} 57$ & $118.4(4)$ \\
\hline $\mathrm{N} 2-\mathrm{C} 31-\mathrm{O} 2$ & $119.0(3)$ & $\mathrm{N} 4-\mathrm{C} 63-\mathrm{O} 4$ & $119.4(3)$ \\
\hline $\mathrm{N} 2-\mathrm{C} 31-\mathrm{C} 23$ & $125.8(3)$ & $\mathrm{N} 4-\mathrm{C} 63-\mathrm{C} 55$ & $125.7(3)$ \\
\hline $\mathrm{O} 2-\mathrm{C} 31-\mathrm{C} 23$ & $115.2(3)$ & $\mathrm{O} 4-\mathrm{C} 63-\mathrm{C} 55$ & $114.9(3)$ \\
\hline $\mathrm{O} 2-\mathrm{C} 32-\mathrm{H} 32 \mathrm{~A}$ & 109.5 & $\mathrm{O} 4-\mathrm{C} 64-\mathrm{H} 64 \mathrm{~A}$ & 109.5 \\
\hline $\mathrm{O} 2-\mathrm{C} 32-\mathrm{H} 32 \mathrm{~B}$ & 109.5 & $\mathrm{O} 4-\mathrm{C} 64-\mathrm{H} 64 \mathrm{~B}$ & 109.5 \\
\hline $\mathrm{H} 32 \mathrm{~A}-\mathrm{C} 32-\mathrm{H} 32 \mathrm{~B}$ & 109.5 & $\mathrm{H} 64 \mathrm{~A}-\mathrm{C} 64-\mathrm{H} 64 \mathrm{~B}$ & 109.5 \\
\hline $\mathrm{O} 2-\mathrm{C} 32-\mathrm{H} 32 \mathrm{C}$ & 109.5 & $\mathrm{O} 4-\mathrm{C} 64-\mathrm{H} 64 \mathrm{C}$ & 109.5 \\
\hline $\mathrm{H} 32 \mathrm{~A}-\mathrm{C} 32-\mathrm{H} 32 \mathrm{C}$ & 109.5 & $\mathrm{H} 64 \mathrm{~A}-\mathrm{C} 64-\mathrm{H} 64 \mathrm{C}$ & 109.5 \\
\hline $\mathrm{H} 32 \mathrm{~B}-\mathrm{C} 32-\mathrm{H} 32 \mathrm{C}$ & 109.5 & $\mathrm{H} 64 \mathrm{~B}-\mathrm{C} 64-\mathrm{H} 64 \mathrm{C}$ & 109.5 \\
\hline $\mathrm{C} 17-\mathrm{C} 1-\mathrm{C} 2-\mathrm{O} 1$ & $52.3(4)$ & $\mathrm{C} 55-\mathrm{C} 33-\mathrm{C} 34-\mathrm{O} 3$ & $-63.8(3)$ \\
\hline $\mathrm{C} 23-\mathrm{C} 1-\mathrm{C} 2-\mathrm{O} 1$ & $-75.9(3)$ & $\mathrm{C} 49-\mathrm{C} 33-\mathrm{C} 34-\mathrm{O} 3$ & $62.5(4)$ \\
\hline $\mathrm{C} 17-\mathrm{C} 1-\mathrm{C} 2-\mathrm{C} 3$ & $169.0(3)$ & $\mathrm{C} 55-\mathrm{C} 33-\mathrm{C} 34-\mathrm{C} 35$ & $52.5(4)$ \\
\hline $\mathrm{C} 23-\mathrm{C} 1-\mathrm{C} 2-\mathrm{C} 3$ & $40.8(4)$ & $\mathrm{C} 49-\mathrm{C} 33-\mathrm{C} 34-\mathrm{C} 35$ & $178.8(3)$ \\
\hline
\end{tabular}




\begin{tabular}{|c|c|}
\hline $\mathrm{C} 17-\mathrm{C} 1-\mathrm{C} 2-\mathrm{C} 7$ & $-68.4(4)$ \\
\hline $\mathrm{C} 23-\mathrm{C} 1-\mathrm{C} 2-\mathrm{C} 7$ & $163.4(3)$ \\
\hline $\mathrm{O} 1-\mathrm{C} 2-\mathrm{C} 3-\mathrm{C} 4$ & $-67.4(3)$ \\
\hline $\mathrm{C} 7-\mathrm{C} 2-\mathrm{C} 3-\mathrm{C} 4$ & $51.6(4)$ \\
\hline $\mathrm{C} 1-\mathrm{C} 2-\mathrm{C} 3-\mathrm{C} 4$ & $175.7(3)$ \\
\hline $\mathrm{C} 5-\mathrm{N} 1-\mathrm{C} 4-\mathrm{C} 3$ & $83.1(4)$ \\
\hline $\mathrm{C} 6-\mathrm{N} 1-\mathrm{C} 4-\mathrm{C} 3$ & $-154.1(3)$ \\
\hline $\mathrm{C} 2-\mathrm{C} 3-\mathrm{C} 4-\mathrm{N} 1$ & $58.7(4)$ \\
\hline $\mathrm{O} 1-\mathrm{C} 2-\mathrm{C} 7-\mathrm{C} 8$ & $-0.5(4)$ \\
\hline $\mathrm{C} 3-\mathrm{C} 2-\mathrm{C} 7-\mathrm{C} 8$ & $-117.3(3)$ \\
\hline $\mathrm{C} 1-\mathrm{C} 2-\mathrm{C} 7-\mathrm{C} 8$ & $118.3(3)$ \\
\hline $\mathrm{O} 1-\mathrm{C} 2-\mathrm{C} 7-\mathrm{C} 16$ & $171.3(3)$ \\
\hline $\mathrm{C} 3-\mathrm{C} 2-\mathrm{C} 7-\mathrm{C} 16$ & $54.5(4)$ \\
\hline $\mathrm{C} 1-\mathrm{C} 2-\mathrm{C} 7-\mathrm{C} 16$ & $-69.8(4)$ \\
\hline $\mathrm{C} 16-\mathrm{C} 7-\mathrm{C} 8-\mathrm{C} 9$ & $-2.8(5)$ \\
\hline $\mathrm{C} 2-\mathrm{C} 7-\mathrm{C} 8-\mathrm{C} 9$ & $169.4(3)$ \\
\hline $\mathrm{C} 7-\mathrm{C} 8-\mathrm{C} 9-\mathrm{C} 10$ & $-0.8(6)$ \\
\hline $\mathrm{C} 8-\mathrm{C} 9-\mathrm{C} 10-\mathrm{C} 11$ & $2.5(6)$ \\
\hline $\mathrm{C} 9-\mathrm{C} 10-\mathrm{C} 11-\mathrm{C} 12$ & $178.7(4)$ \\
\hline $\mathrm{C} 9-\mathrm{C} 10-\mathrm{C} 11-\mathrm{C} 16$ & $-0.6(6)$ \\
\hline $\mathrm{C} 10-\mathrm{C} 11-\mathrm{C} 12-\mathrm{C} 13$ & $-176.8(5)$ \\
\hline $\mathrm{C} 16-\mathrm{C} 11-\mathrm{C} 12-\mathrm{C} 13$ & $2.4(7)$ \\
\hline $\mathrm{C} 11-\mathrm{C} 12-\mathrm{C} 13-\mathrm{C} 14$ & $-0.7(8)$ \\
\hline $\mathrm{C} 12-\mathrm{C} 13-\mathrm{C} 14-\mathrm{C} 15$ & $-1.2(8)$ \\
\hline $\mathrm{C} 13-\mathrm{C} 14-\mathrm{C} 15-\mathrm{C} 16$ & $1.5(7)$ \\
\hline $\mathrm{C} 14-\mathrm{C} 15-\mathrm{C} 16-\mathrm{C} 11$ & $0.2(6)$ \\
\hline $\mathrm{C} 14-\mathrm{C} 15-\mathrm{C} 16-\mathrm{C} 7$ & $-179.7(4)$ \\
\hline $\mathrm{C} 12-\mathrm{C} 11-\mathrm{C} 16-\mathrm{C} 15$ & $-2.1(6)$ \\
\hline $\mathrm{C} 10-\mathrm{C} 11-\mathrm{C} 16-\mathrm{C} 15$ & $177.2(4)$ \\
\hline $\mathrm{C} 12-\mathrm{C} 11-\mathrm{C} 16-\mathrm{C} 7$ & $177.8(4)$ \\
\hline $\mathrm{C} 10-\mathrm{C} 11-\mathrm{C} 16-\mathrm{C} 7$ & $-2.9(5)$ \\
\hline $\mathrm{C} 8-\mathrm{C} 7-\mathrm{C} 16-\mathrm{C} 15$ & $-175.6(4)$ \\
\hline $\mathrm{C} 2-\mathrm{C} 7-\mathrm{C} 16-\mathrm{C} 15$ & $12.6(6)$ \\
\hline $\mathrm{C} 8-\mathrm{C} 7-\mathrm{C} 16-\mathrm{C} 11$ & $4.6(5)$ \\
\hline $\mathrm{C} 2-\mathrm{C} 7-\mathrm{C} 16-\mathrm{C} 11$ & $-167.3(3)$ \\
\hline $\mathrm{C} 23-\mathrm{C} 1-\mathrm{C} 17-\mathrm{C} 22$ & $81.5(4)$ \\
\hline $\mathrm{C} 2-\mathrm{C} 1-\mathrm{C} 17-\mathrm{C} 22$ & $-47.9(5)$ \\
\hline $\mathrm{C} 23-\mathrm{C} 1-\mathrm{C} 17-\mathrm{C} 18$ & $-93.4(4)$ \\
\hline $\mathrm{C} 2-\mathrm{C} 1-\mathrm{C} 17-\mathrm{C} 18$ & $137.3(3)$ \\
\hline $\mathrm{C} 22-\mathrm{C} 17-\mathrm{C} 18-\mathrm{C} 19$ & $-1.0(6)$ \\
\hline $\mathrm{C} 1-\mathrm{C} 17-\mathrm{C} 18-\mathrm{C} 19$ & $174.2(4)$ \\
\hline $\mathrm{C} 17-\mathrm{C} 18-\mathrm{C} 19-\mathrm{C} 20$ & $0.5(6)$ \\
\hline $\mathrm{C} 18-\mathrm{C} 19-\mathrm{C} 20-\mathrm{C} 21$ & $0.1(7)$ \\
\hline $\mathrm{C} 19-\mathrm{C} 20-\mathrm{C} 21-\mathrm{C} 22$ & $-0.1(7)$ \\
\hline $\mathrm{C} 20-\mathrm{C} 21-\mathrm{C} 22-\mathrm{C} 17$ & $-0.4(7)$ \\
\hline $\mathrm{C} 18-\mathrm{C} 17-\mathrm{C} 22-\mathrm{C} 21$ & $0.9(6)$ \\
\hline $\mathrm{C} 1-\mathrm{C} 17-\mathrm{C} 22-\mathrm{C} 21$ & $-173.9(4)$ \\
\hline $\mathrm{C} 17-\mathrm{C} 1-\mathrm{C} 23-\mathrm{C} 24$ & $-54.5(4)$ \\
\hline
\end{tabular}

\begin{tabular}{|c|c|}
\hline $\mathrm{C} 55-\mathrm{C} 33-\mathrm{C} 34-\mathrm{C} 39$ & $177.2(3)$ \\
\hline $\mathrm{C} 49-\mathrm{C} 33-\mathrm{C} 34-\mathrm{C} 39$ & $-56.5(4)$ \\
\hline $\mathrm{O} 3-\mathrm{C} 34-\mathrm{C} 35-\mathrm{C} 36$ & $-66.6(4)$ \\
\hline $\mathrm{C} 39-\mathrm{C} 34-\mathrm{C} 35-\mathrm{C} 36$ & $53.1(4)$ \\
\hline $\mathrm{C} 33-\mathrm{C} 34-\mathrm{C} 35-\mathrm{C} 36$ & $177.0(3)$ \\
\hline $\mathrm{C} 37-\mathrm{N} 3-\mathrm{C} 36-\mathrm{C} 35$ & $84.3(4)$ \\
\hline $\mathrm{C} 38-\mathrm{N} 3-\mathrm{C} 36-\mathrm{C} 35$ & $-152.0(3)$ \\
\hline $\mathrm{C} 34-\mathrm{C} 35-\mathrm{C} 36-\mathrm{N} 3$ & $63.0(4)$ \\
\hline $\mathrm{O} 3-\mathrm{C} 34-\mathrm{C} 39-\mathrm{C} 40$ & $-5.9(5)$ \\
\hline $\mathrm{C} 35-\mathrm{C} 34-\mathrm{C} 39-\mathrm{C} 40$ & $-123.9(4)$ \\
\hline $\mathrm{C} 33-\mathrm{C} 34-\mathrm{C} 39-\mathrm{C} 40$ & $111.6(4)$ \\
\hline $\mathrm{O} 3-\mathrm{C} 34-\mathrm{C} 39-\mathrm{C} 48$ & $171.9(3)$ \\
\hline $\mathrm{C} 35-\mathrm{C} 34-\mathrm{C} 39-\mathrm{C} 48$ & $53.8(4)$ \\
\hline $\mathrm{C} 33-\mathrm{C} 34-\mathrm{C} 39-\mathrm{C} 48$ & $-70.7(4)$ \\
\hline $\mathrm{C} 48-\mathrm{C} 39-\mathrm{C} 40-\mathrm{C} 41$ & $-1.6(7)$ \\
\hline $\mathrm{C} 34-\mathrm{C} 39-\mathrm{C} 40-\mathrm{C} 41$ & $176.3(4)$ \\
\hline $\mathrm{C} 39-\mathrm{C} 40-\mathrm{C} 41-\mathrm{C} 42$ & $-1.6(8)$ \\
\hline $\mathrm{C} 40-\mathrm{C} 41-\mathrm{C} 42-\mathrm{C} 43$ & $1.5(8)$ \\
\hline $\mathrm{C} 41-\mathrm{C} 42-\mathrm{C} 43-\mathrm{C} 44$ & $-179.2(5)$ \\
\hline $\mathrm{C} 41-\mathrm{C} 42-\mathrm{C} 43-\mathrm{C} 48$ & $1.6(7)$ \\
\hline $\mathrm{C} 42-\mathrm{C} 43-\mathrm{C} 44-\mathrm{C} 45$ & $-178.0(4)$ \\
\hline $\mathrm{C} 48-\mathrm{C} 43-\mathrm{C} 44-\mathrm{C} 45$ & $1.2(6)$ \\
\hline $\mathrm{C} 43-\mathrm{C} 44-\mathrm{C} 45-\mathrm{C} 46$ & $1.5(6)$ \\
\hline $\mathrm{C} 44-\mathrm{C} 45-\mathrm{C} 46-\mathrm{C} 47$ & $-2.5(6)$ \\
\hline $\mathrm{C} 45-\mathrm{C} 46-\mathrm{C} 47-\mathrm{C} 48$ & $0.8(6)$ \\
\hline $\mathrm{C} 46-\mathrm{C} 47-\mathrm{C} 48-\mathrm{C} 43$ & $1.9(5)$ \\
\hline $\mathrm{C} 46-\mathrm{C} 47-\mathrm{C} 48-\mathrm{C} 39$ & $-177.0(3)$ \\
\hline $\mathrm{C} 42-\mathrm{C} 43-\mathrm{C} 48-\mathrm{C} 47$ & $176.4(4)$ \\
\hline $\mathrm{C} 44-\mathrm{C} 43-\mathrm{C} 48-\mathrm{C} 47$ & $-2.8(5)$ \\
\hline $\mathrm{C} 42-\mathrm{C} 43-\mathrm{C} 48-\mathrm{C} 39$ & $-4.7(5)$ \\
\hline $\mathrm{C} 44-\mathrm{C} 43-\mathrm{C} 48-\mathrm{C} 39$ & $176.2(3)$ \\
\hline $\mathrm{C} 40-\mathrm{C} 39-\mathrm{C} 48-\mathrm{C} 47$ & $-176.6(4)$ \\
\hline $\mathrm{C} 34-\mathrm{C} 39-\mathrm{C} 48-\mathrm{C} 47$ & $5.7(5)$ \\
\hline $\mathrm{C} 40-\mathrm{C} 39-\mathrm{C} 48-\mathrm{C} 43$ & $4.5(5)$ \\
\hline $\mathrm{C} 34-\mathrm{C} 39-\mathrm{C} 48-\mathrm{C} 43$ & $-173.2(3)$ \\
\hline $\mathrm{C} 55-\mathrm{C} 33-\mathrm{C} 49-\mathrm{C} 50$ & $-91.5(4)$ \\
\hline $\mathrm{C} 34-\mathrm{C} 33-\mathrm{C} 49-\mathrm{C} 50$ & $139.4(3)$ \\
\hline $\mathrm{C} 55-\mathrm{C} 33-\mathrm{C} 49-\mathrm{C} 54$ & $82.5(4)$ \\
\hline $\mathrm{C} 34-\mathrm{C} 33-\mathrm{C} 49-\mathrm{C} 54$ & $-46.6(5)$ \\
\hline $\mathrm{C} 54-\mathrm{C} 49-\mathrm{C} 50-\mathrm{C} 51$ & $-0.6(6)$ \\
\hline $\mathrm{C} 33-\mathrm{C} 49-\mathrm{C} 50-\mathrm{C} 51$ & $173.8(4)$ \\
\hline $\mathrm{C} 49-\mathrm{C} 50-\mathrm{C} 51-\mathrm{C} 52$ & $0.8(6)$ \\
\hline $\mathrm{C} 50-\mathrm{C} 51-\mathrm{C} 52-\mathrm{C} 53$ & $-0.5(7)$ \\
\hline $\mathrm{C} 51-\mathrm{C} 52-\mathrm{C} 53-\mathrm{C} 54$ & $-0.1(7)$ \\
\hline $\mathrm{C} 52-\mathrm{C} 53-\mathrm{C} 54-\mathrm{C} 49$ & $0.4(6)$ \\
\hline $\mathrm{C} 50-\mathrm{C} 49-\mathrm{C} 54-\mathrm{C} 53$ & $0.0(6)$ \\
\hline $\mathrm{C} 33-\mathrm{C} 49-\mathrm{C} 54-\mathrm{C} 53$ & $-174.0(4)$ \\
\hline $\mathrm{C} 49-\mathrm{C} 33-\mathrm{C} 55-\mathrm{C} 56$ & $-63.1(4)$ \\
\hline
\end{tabular}




$\begin{array}{ll}\mathrm{C} 2-\mathrm{C} 1-\mathrm{C} 23-\mathrm{C} 24 & 77.3(4) \\ \mathrm{C} 17-\mathrm{C} 1-\mathrm{C} 23-\mathrm{C} 31 & 126.0(3) \\ \mathrm{C} 2-\mathrm{C} 1-\mathrm{C} 23-\mathrm{C} 31 & -102.2(3) \\ \mathrm{C} 31-\mathrm{C} 23-\mathrm{C} 24-\mathrm{C} 25 & -0.6(5) \\ \mathrm{C} 1-\mathrm{C} 23-\mathrm{C} 24-\mathrm{C} 25 & 179.8(3) \\ \mathrm{C} 23-\mathrm{C} 24-\mathrm{C} 25-\mathrm{C} 26 & 178.3(3) \\ \mathrm{C} 23-\mathrm{C} 24-\mathrm{C} 25-\mathrm{C} 30 & -1.1(5) \\ \mathrm{C} 30-\mathrm{C} 25-\mathrm{C} 26-\mathrm{C} 27 & 0.1(5) \\ \mathrm{C} 24-\mathrm{C} 25-\mathrm{C} 26-\mathrm{C} 27 & -179.3(3) \\ \mathrm{C} 25-\mathrm{C} 26-\mathrm{C} 27-\mathrm{C} 28 & 0.1(5) \\ \mathrm{C} 25-\mathrm{C} 26-\mathrm{C} 27-\mathrm{B} 1 & -180.0(3) \\ \mathrm{C} 26-\mathrm{C} 27-\mathrm{C} 28-\mathrm{C} 29 & -0.3(6) \\ \mathrm{B} 1-\mathrm{C} 27-\mathrm{C} 28-\mathrm{C} 29 & 179.8(3) \\ \mathrm{C} 27-\mathrm{C} 28-\mathrm{C} 29-\mathrm{C} 30 & 0.2(6) \\ \mathrm{C} 31-\mathrm{N} 2-\mathrm{C} 30-\mathrm{C} 29 & -177.9(3) \\ \mathrm{C} 31-\mathrm{N} 2-\mathrm{C} 30-\mathrm{C} 25 & -0.3(5) \\ \mathrm{C} 28-\mathrm{C} 29-\mathrm{C} 30-\mathrm{N} 2 & 177.8(3) \\ \mathrm{C} 28-\mathrm{C} 29-\mathrm{C} 30-\mathrm{C} 25 & 0.0(5) \\ \mathrm{C} 26-\mathrm{C} 25-\mathrm{C} 30-\mathrm{N} 2 & -177.8(3) \\ \mathrm{C} 24-\mathrm{C} 25-\mathrm{C} 30-\mathrm{N} 2 & 1.6(5) \\ \mathrm{C} 26-\mathrm{C} 25-\mathrm{C} 30-\mathrm{C} 29 & -0.2(5) \\ \mathrm{C} 24-\mathrm{C} 25-\mathrm{C} 30-\mathrm{C} 29 & 179.3(3) \\ \mathrm{C} 30-\mathrm{N} 2-\mathrm{C} 31-\mathrm{O} 2 & 176.5(3) \\ \mathrm{C} 30-\mathrm{N} 2-\mathrm{C} 31-\mathrm{C} 23 & -1.7(5) \\ \mathrm{C} 32-\mathrm{O} 2-\mathrm{C} 31-\mathrm{N} 2 & 0.6(5) \\ \mathrm{C} 32-\mathrm{O} 2-\mathrm{C} 31-\mathrm{C} 23 & 179.0(3) \\ \mathrm{C} 24-\mathrm{C} 23-\mathrm{C} 31-\mathrm{N} 2 & 2.1(5) \\ \mathrm{C} 1-\mathrm{C} 23-\mathrm{C} 31-\mathrm{N} 2 & -178.3(3) \\ \mathrm{C} 24-\mathrm{C} 23-\mathrm{C} 31-\mathrm{O} 2 & -176.1(3) \\ \mathrm{C} 1-\mathrm{C} 23-\mathrm{C} 31-\mathrm{O} 2 & 3.5(4) \\ & \end{array}$

$$
\begin{aligned}
& \mathrm{C} 34-\mathrm{C} 33-\mathrm{C} 55-\mathrm{C} 56 \\
& \mathrm{C} 49-\mathrm{C} 33-\mathrm{C} 55-\mathrm{C} 63 \\
& \mathrm{C} 34-\mathrm{C} 33-\mathrm{C} 55-\mathrm{C} 63 \\
& \text { C63-C55-C56-C57 } \\
& \mathrm{C} 33-\mathrm{C} 55-\mathrm{C} 56-\mathrm{C} 57 \\
& \mathrm{C} 55-\mathrm{C} 56-\mathrm{C} 57-\mathrm{C} 58 \\
& \text { C55-C56-C57-C62 } \\
& \text { C62-C57-C58-C59 } \\
& \text { C56-C } 57-\mathrm{C} 58-\mathrm{C} 59 \\
& \mathrm{C} 57-\mathrm{C} 58-\mathrm{C} 59-\mathrm{C} 60 \\
& \mathrm{C} 57-\mathrm{C} 58-\mathrm{C} 59-\mathrm{Br} 2 \\
& \mathrm{C} 58-\mathrm{C} 59-\mathrm{C} 60-\mathrm{C} 61 \\
& \mathrm{Br} 2-\mathrm{C} 59-\mathrm{C} 60-\mathrm{C} 61 \\
& \mathrm{C} 59-\mathrm{C} 60-\mathrm{C} 61-\mathrm{C} 62 \\
& \text { C63-N4-C62-C61 } \\
& \text { C63-N4-C62-C57 } \\
& \mathrm{C} 60-\mathrm{C} 61-\mathrm{C} 62-\mathrm{N} 4 \\
& \text { C60-C61-C62-C57 } \\
& \mathrm{C} 58-\mathrm{C} 57-\mathrm{C} 62-\mathrm{N} 4 \\
& \mathrm{C} 56-\mathrm{C} 57-\mathrm{C} 62-\mathrm{N} 4 \\
& \text { C58-C57-C62-C61 } \\
& \mathrm{C} 56-\mathrm{C} 57-\mathrm{C} 62-\mathrm{C} 61 \\
& \text { C62-N4-C63-O4 } \\
& \mathrm{C} 62-\mathrm{N} 4-\mathrm{C} 63-\mathrm{C} 55 \\
& \text { C64-O4-C63-N4 } \\
& \mathrm{C} 64-\mathrm{O} 4-\mathrm{C} 63-\mathrm{C} 55 \\
& \mathrm{C} 56-\mathrm{C} 55-\mathrm{C} 63-\mathrm{N} 4 \\
& \mathrm{C} 33-\mathrm{C} 55-\mathrm{C} 63-\mathrm{N} 4 \\
& \mathrm{C} 56-\mathrm{C} 55-\mathrm{C} 63-\mathrm{O} 4 \\
& \mathrm{C} 33-\mathrm{C} 55-\mathrm{C} 63-\mathrm{O} 4
\end{aligned}
$$

$67.1(4)$

$112.6(3)$

$-117.2(3)$

$-2.4(5)$

$173.4(3)$

$179.8(3)$

$-2.1(5)$

$-1.7(5)$

$176.3(3)$

$-0.8(5)$

$180.0(3)$

1.7 (6)

$-179.1(3)$

$-0.1(6)$

$177.2(3)$

$-2.6(5)$

177.9 (3)

$-2.4(5)$

$-177.0(3)$

$4.8(5)$

$3.3(5)$

$-174.9(3)$

$178.1(3)$

$-2.6(5)$

$-0.1(5)$

$-179.4(3)$

$5.1(5)$

$-170.9(3)$

$-175.6(3)$

$8.4(5)$

Hydrogen-bond geometry $\left(\AA,{ }^{\circ}\right)$

\begin{tabular}{lllll}
\hline$D-\mathrm{H} \cdots A$ & $D-\mathrm{H}$ & $\mathrm{H} \cdots A$ & $D \cdots A$ & $D-\mathrm{H} \cdots A$ \\
\hline $\mathrm{O} 1-\mathrm{H} 1 O \cdots \mathrm{N} 1$ & 0.84 & 1.94 & $2.696(4)$ & 150 \\
$\mathrm{C} 1-\mathrm{H} 1 \cdots \mathrm{O} 2$ & 1.00 & 2.24 & $2.763(4)$ & 111 \\
$\mathrm{O} 3-\mathrm{H} 3 O \cdots \mathrm{N} 3$ & 0.84 & 1.93 & $2.685(4)$ & 149 \\
$\mathrm{C} 33-\mathrm{H} 33 \cdots \mathrm{O} 4$ & 1.00 & 2.23 & $2.773(4)$ & 112 \\
\hline
\end{tabular}

[4-(6-Bromo-2-methoxyquinolin-3-yl)-3-hydroxy-3-(naphthalen-1-yl)-4-phenylbutyl]dimethylazanium 3carboxyprop-2-enoate (2)

\section{Crystal data}

$\mathrm{C}_{32} \mathrm{H}_{32} \mathrm{BrN}_{2} \mathrm{O}_{2}^{+} \cdot \mathrm{C}_{4} \mathrm{H}_{3} \mathrm{O}_{4}^{-}$

$M_{r}=671.57$

Monoclinic, $P 2_{1}$

$a=16.4556$ (6) $\AA$

$b=10.3205$ (3) $\AA$

$$
\begin{aligned}
& c=20.1636(8) \AA \\
& \beta=109.1832(15)^{\circ} \\
& V=3234.2(2) \AA^{3} \\
& Z=4 \\
& F(000)=1392
\end{aligned}
$$


$D_{\mathrm{x}}=1.379 \mathrm{Mg} \mathrm{m}^{-3}$

Mo $K \alpha$ radiation, $\lambda=0.71073 \AA$

Cell parameters from 9891 reflections

$\theta=2.9-31.4^{\circ}$

\section{Data collection}

Bruker D8 Quest

diffractometer with PhotonII charge-integrating pixel array detector (CPAD)

Radiation source: fine focus sealed tube X-ray source

Triumph curved graphite crystal monochromator

Detector resolution: 7.4074 pixels $\mathrm{mm}^{-1}$

$\omega$ and phi scans

\section{Refinement}

Refinement on $F^{2}$

Least-squares matrix: full

$R\left[F^{2}>2 \sigma\left(F^{2}\right)\right]=0.043$

$w R\left(F^{2}\right)=0.117$

$S=1.06$

24622 reflections

837 parameters

1 restraint

Primary atom site location: structure-invariant direct methods

Secondary atom site location: difference Fourier map $\mu=1.32 \mathrm{~mm}^{-1}$

$T=150 \mathrm{~K}$

Block, colourless

$0.45 \times 0.37 \times 0.17 \mathrm{~mm}$

Absorption correction: multi-scan

(SADABS2016; Krause et al., 2015)

$T_{\min }=0.438, T_{\max }=0.495$

115858 measured reflections

24622 independent reflections

18572 reflections with $I>2 \sigma(I)$

$R_{\text {int }}=0.040$

$\theta_{\max }=33.2^{\circ}, \theta_{\min }=2.3^{\circ}$

$h=-25 \rightarrow 25$

$k=-15 \rightarrow 15$

$l=-28 \rightarrow 31$

Hydrogen site location: mixed

$\mathrm{H}$ atoms treated by a mixture of independent and constrained refinement

$w=1 /\left[\sigma^{2}\left(F_{0}^{2}\right)+(0.0609 P)^{2}+0.4737 P\right]$

where $P=\left(F_{\mathrm{o}}^{2}+2 F_{\mathrm{c}}{ }^{2}\right) / 3$

$(\Delta / \sigma)_{\max }=0.001$

$\Delta \rho_{\max }=1.23 \mathrm{e} \AA^{-3}$

$\Delta \rho_{\min }=-1.28$ e $\AA^{-3}$

Absolute structure: Flack $x$ determined using 7327 quotients $[(\mathrm{I}+)-(\mathrm{I}-)] /[(\mathrm{I}+)+(\mathrm{I}-)]$ (Parsons et al., 2013)

Absolute structure parameter: -0.0144 (14)

\section{Special details}

Geometry. All esds (except the esd in the dihedral angle between two 1.s. planes) are estimated using the full covariance matrix. The cell esds are taken into account individually in the estimation of esds in distances, angles and torsion angles; correlations between esds in cell parameters are only used when they are defined by crystal symmetry. An approximate (isotropic) treatment of cell esds is used for estimating esds involving l.s. planes.

Fractional atomic coordinates and isotropic or equivalent isotropic displacement parameters $\left(\AA^{2}\right)$

\begin{tabular}{lllll}
\hline & $x$ & $y$ & $z$ & $U_{\text {iso }} * / U_{\text {eq }}$ \\
\hline Br1A & $0.58678(3)$ & $0.47935(4)$ & $0.22528(2)$ & $0.06533(14)$ \\
O1A & $0.82483(12)$ & $-0.01085(19)$ & $0.51792(10)$ & $0.0278(3)$ \\
H1AB & $0.828(2)$ & $0.039(4)$ & $0.485(2)$ & $0.042 *$ \\
O2A & $0.77472(16)$ & $0.3652(3)$ & $0.64605(13)$ & $0.0484(6)$ \\
O3A & $0.93472(13)$ & $0.2628(2)$ & $0.42487(10)$ & $0.0344(4)$ \\
O4A & $0.84664(14)$ & $0.09248(19)$ & $0.40198(9)$ & $0.0349(4)$ \\
O5A & $0.81194(14)$ & $0.52568(19)$ & $0.20888(11)$ & $0.0350(4)$ \\
H5A & $0.763(3)$ & $0.555(4)$ & $0.164(2)$ & $0.052^{*}$ \\
O6A & $0.76176(14)$ & $0.3298(2)$ & $0.16731(10)$ & $0.0373(4)$ \\
N1A & $1.03839(12)$ & $0.1521(2)$ & $0.53959(11)$ & $0.0244(4)$ \\
H1AN & $0.993(2)$ & $0.179(4)$ & $0.4961(19)$ & $0.029 *$ \\
N2A & $0.73312(15)$ & $0.4780(3)$ & $0.54157(16)$ & $0.0405(6)$
\end{tabular}




\begin{tabular}{|c|c|c|c|c|}
\hline $\mathrm{C} 1 \mathrm{~A}$ & $0.75797(15)$ & $0.1203(3)$ & $0.58725(12)$ & $0.0253(4)$ \\
\hline H1A & 0.771592 & 0.149992 & 0.636934 & $0.030 *$ \\
\hline $\mathrm{C} 2 \mathrm{~A}$ & $0.84239(15)$ & 0.0560 (2) & $0.58297(13)$ & $0.0239(4)$ \\
\hline $\mathrm{C} 3 \mathrm{~A}$ & $0.91264(15)$ & $0.1580(2)$ & $0.58576(14)$ & $0.0262(4)$ \\
\hline H3AA & 0.892408 & 0.215552 & 0.544294 & $0.031^{*}$ \\
\hline H3AB & 0.923897 & 0.211845 & 0.628457 & $0.031 *$ \\
\hline $\mathrm{C} 4 \mathrm{~A}$ & $0.99581(16)$ & $0.0896(3)$ & $0.58663(15)$ & $0.0314(5)$ \\
\hline H4AA & 1.036546 & 0.089161 & 0.635301 & $0.038^{*}$ \\
\hline H4AB & 0.982420 & -0.001652 & 0.571907 & $0.038^{*}$ \\
\hline C5A & $1.0918(2)$ & 0.2647 (3) & $0.57295(16)$ & $0.0404(7)$ \\
\hline H5AA & 1.115756 & 0.304971 & 0.539441 & $0.061^{*}$ \\
\hline H5AB & 1.138756 & 0.235812 & 0.614397 & $0.061^{*}$ \\
\hline H5AC & 1.056186 & 0.327915 & 0.587129 & $0.061^{*}$ \\
\hline C6A & $1.0901(2)$ & $0.0563(3)$ & $0.5157(2)$ & $0.0441(7)$ \\
\hline H6AA & 1.051580 & -0.006000 & 0.483812 & $0.066^{*}$ \\
\hline H6AB & 1.128727 & 0.010308 & 0.556399 & $0.066^{*}$ \\
\hline H6AC & 1.124180 & 0.101322 & 0.491018 & $0.066^{*}$ \\
\hline C7A & $0.87611(15)$ & $-0.0436(3)$ & 0.64224 (14) & $0.0283(5)$ \\
\hline $\mathrm{C} 8 \mathrm{~A}$ & $0.8828(2)$ & $-0.1710(3)$ & $0.62370(17)$ & $0.0364(6)$ \\
\hline $\mathrm{H} 8 \mathrm{~A}$ & 0.864922 & -0.192956 & 0.575279 & $0.044 *$ \\
\hline C9A & $0.9150(2)$ & $-0.2694(3)$ & 0.6737 (2) & $0.0479(8)$ \\
\hline H9A & 0.918640 & -0.355840 & 0.658637 & $0.058^{*}$ \\
\hline $\mathrm{C} 10 \mathrm{~A}$ & 0.9408 (2) & $-0.2416(4)$ & $0.7432(2)$ & $0.0483(8)$ \\
\hline $\mathrm{H} 10 \mathrm{~A}$ & 0.961810 & -0.308627 & 0.776728 & $0.058^{*}$ \\
\hline $\mathrm{C} 11 \mathrm{~A}$ & 0.93654 (19) & $-0.1126(3)$ & $0.76586(16)$ & $0.0407(7)$ \\
\hline $\mathrm{C} 12 \mathrm{~A}$ & $0.9655(2)$ & $-0.0838(5)$ & 0.83857 (17) & $0.0526(9)$ \\
\hline $\mathrm{H} 12 \mathrm{~A}$ & 0.986902 & -0.151708 & 0.871500 & $0.063^{*}$ \\
\hline $\mathrm{C} 13 \mathrm{~A}$ & 0.9633 (3) & $0.0384(5)$ & $0.86202(19)$ & $0.0597(11)$ \\
\hline $\mathrm{H} 13 \mathrm{~A}$ & 0.983221 & 0.055843 & 0.911000 & $0.072 *$ \\
\hline $\mathrm{C} 14 \mathrm{~A}$ & 0.9313 (2) & 0.1395 (4) & $0.81378(16)$ & 0.0479 (8) \\
\hline $\mathrm{H} 14 \mathrm{~A}$ & 0.929246 & 0.225084 & 0.830529 & $0.058^{*}$ \\
\hline $\mathrm{C} 15 \mathrm{~A}$ & 0.90318 (19) & $0.1164(3)$ & $0.74297(15)$ & $0.0357(6)$ \\
\hline H15A & 0.882490 & 0.186537 & 0.711397 & $0.043^{*}$ \\
\hline C16A & $0.90432(16)$ & $-0.0111(3)$ & 0.71559 (14) & $0.0316(5)$ \\
\hline $\mathrm{C} 17 \mathrm{~A}$ & $0.67859(15)$ & $0.0327(3)$ & $0.57224(13)$ & $0.0277(5)$ \\
\hline C18A & $0.6128(2)$ & 0.0787 (4) & $0.5946(2)$ & $0.0497(8)$ \\
\hline $\mathrm{H} 18 \mathrm{~A}$ & 0.620591 & 0.157373 & 0.620373 & $0.060 *$ \\
\hline C19A & $0.5355(2)$ & 0.0117 (4) & 0.5799 (2) & $0.0569(10)$ \\
\hline H19A & 0.490525 & 0.046866 & 0.594292 & $0.068 *$ \\
\hline $\mathrm{C} 20 \mathrm{~A}$ & 0.52354 (19) & $-0.1032(4)$ & $0.54536(18)$ & $0.0475(8)$ \\
\hline $\mathrm{H} 20 \mathrm{~A}$ & 0.470901 & -0.149129 & 0.535924 & $0.057^{*}$ \\
\hline $\mathrm{C} 21 \mathrm{~A}$ & 0.5879 (2) & $-0.1517(4)$ & $0.52446(18)$ & $0.0487(8)$ \\
\hline $\mathrm{H} 21 \mathrm{~A}$ & 0.580561 & -0.232974 & 0.501099 & $0.058^{*}$ \\
\hline $\mathrm{C} 22 \mathrm{~A}$ & $0.66510(19)$ & $-0.0831(4)$ & $0.53697(17)$ & $0.0461(8)$ \\
\hline $\mathrm{H} 22 \mathrm{~A}$ & 0.708730 & -0.117411 & 0.520735 & $0.055^{*}$ \\
\hline $\mathrm{C} 23 \mathrm{~A}$ & $0.73471(15)$ & $0.2414(2)$ & $0.54251(14)$ & $0.0272(5)$ \\
\hline $\mathrm{C} 24 \mathrm{~A}$ & $0.70146(15)$ & $0.2396(2)$ & $0.47090(14)$ & $0.0264(5)$ \\
\hline $\mathrm{H} 24 \mathrm{~A}$ & 0.689599 & 0.159083 & 0.446837 & $0.032 *$ \\
\hline
\end{tabular}




\begin{tabular}{|c|c|c|c|c|}
\hline $\mathrm{C} 25 \mathrm{~A}$ & $0.68451(16)$ & 0.3567 (3) & $0.43217(16)$ & $0.0307(5)$ \\
\hline $\mathrm{C} 26 \mathrm{~A}$ & $0.64932(18)$ & $0.3588(3)$ & $0.35808(16)$ & $0.0360(6)$ \\
\hline $\mathrm{H} 26 \mathrm{~A}$ & 0.635255 & 0.280285 & 0.332208 & $0.043 *$ \\
\hline $\mathrm{C} 27 \mathrm{~A}$ & $0.6359(2)$ & $0.4759(3)$ & $0.32424(19)$ & $0.0442(7)$ \\
\hline $\mathrm{C} 28 \mathrm{~A}$ & $0.6564(2)$ & $0.5939(3)$ & $0.3598(2)$ & $0.0473(8)$ \\
\hline $\mathrm{H} 28 \mathrm{~A}$ & 0.648076 & 0.673357 & 0.334619 & $0.057^{*}$ \\
\hline C29A & $0.68871(19)$ & $0.5927(3)$ & $0.4315(2)$ & $0.0455(8)$ \\
\hline $\mathrm{H} 29 \mathrm{~A}$ & 0.702150 & 0.672378 & 0.456212 & $0.055^{*}$ \\
\hline $\mathrm{C} 30 \mathrm{~A}$ & $0.70257(16)$ & 0.4748 (3) & $0.46960(17)$ & $0.0348(6)$ \\
\hline C31A & 0.74691 (17) & $0.3681(3)$ & $0.57451(17)$ & $0.0361(6)$ \\
\hline $\mathrm{C} 32 \mathrm{~A}$ & $0.7682(3)$ & $0.4825(5)$ & $0.6821(2)$ & $0.0641(12)$ \\
\hline $\mathrm{H} 32 \mathrm{~A}$ & 0.800334 & 0.551543 & 0.668372 & $0.096^{*}$ \\
\hline $\mathrm{H} 32 \mathrm{~B}$ & 0.792178 & 0.468439 & 0.732896 & $0.096^{*}$ \\
\hline $\mathrm{H} 32 \mathrm{C}$ & 0.707587 & 0.507649 & 0.669651 & $0.096^{*}$ \\
\hline $\mathrm{C} 33 \mathrm{~A}$ & $0.87369(16)$ & 0.1975 (3) & $0.38480(12)$ & $0.0264(4)$ \\
\hline C34A & $0.82928(16)$ & $0.2472(3)$ & $0.31203(13)$ & $0.0267(5)$ \\
\hline H34A & 0.779369 & 0.202920 & 0.283576 & $0.032 *$ \\
\hline C35A & $0.85588(16)$ & $0.3491(3)$ & 0.28539 (13) & $0.0269(5)$ \\
\hline $\mathrm{H} 35 \mathrm{~A}$ & 0.907626 & 0.391592 & 0.311789 & $0.032 *$ \\
\hline $\mathrm{C} 36 \mathrm{~A}$ & 0.80515 (16) & $0.3986(2)$ & $0.21389(13)$ & $0.0280(5)$ \\
\hline Br1B & $0.03711(2)$ & $0.42464(3)$ & $-0.28802(2)$ & 0.04720 \\
\hline O1B & $0.33662(11)$ & $0.00508(18)$ & $0.02269(9)$ & 0.0267 (3) \\
\hline H1B & $0.327(2)$ & $0.060(4)$ & $-0.013(2)$ & $0.040 *$ \\
\hline $\mathrm{O} 2 \mathrm{~B}$ & $0.27505(14)$ & $0.3816(2)$ & 0.13215 (11) & $0.0392(5)$ \\
\hline O3B & $0.57136(13)$ & $0.7488(3)$ & $0.07837(11)$ & $0.0460(6)$ \\
\hline O4B & 0.68777 (12) & $0.62216(19)$ & $0.10440(9)$ & 0.0294 (4) \\
\hline O5B & $0.64680(14)$ & $1.0698(2)$ & $0.27053(14)$ & $0.0453(5)$ \\
\hline O6B & $0.75267(14)$ & $0.9246(2)$ & $0.31105(10)$ & $0.0398(5)$ \\
\hline H6B & $0.771(3)$ & $0.989(5)$ & $0.339(2)$ & $0.060 *$ \\
\hline N1B & $0.53663(13)$ & 0.1951 (2) & 0.04729 (12) & 0.0283 (4) \\
\hline $\mathrm{H} 1 \mathrm{BN}$ & $0.502(2)$ & $0.191(4)$ & $0.006(2)$ & $0.034 *$ \\
\hline N2B & $0.22630(13)$ & $0.4745(2)$ & $0.02299(12)$ & $0.0303(4)$ \\
\hline C1B & $0.25543(14)$ & $0.1260(2)$ & $0.08575(12)$ & $0.0230(4)$ \\
\hline H1BA & 0.264502 & 0.161026 & 0.133894 & $0.028^{*}$ \\
\hline $\mathrm{C} 2 \mathrm{~B}$ & 0.34548 (14) & 0.0737 (2) & $0.08604(12)$ & $0.0229(4)$ \\
\hline $\mathrm{C} 3 \mathrm{~B}$ & $0.40925(15)$ & 0.1858 (3) & $0.08942(14)$ & $0.0267(5)$ \\
\hline H3BA & 0.387922 & 0.239023 & 0.046308 & $0.032 *$ \\
\hline H3BB & 0.413490 & 0.242027 & 0.130194 & $0.032 *$ \\
\hline C4B & $0.49810(15)$ & $0.1312(3)$ & 0.09628 (14) & $0.0294(5)$ \\
\hline H4BA & 0.536885 & 0.143220 & 0.145135 & $0.035^{*}$ \\
\hline H4BB & 0.493009 & 0.036984 & 0.086459 & $0.035^{*}$ \\
\hline C5B & $0.55983(19)$ & 0.3328 (3) & $0.06465(17)$ & $0.0405(6)$ \\
\hline H5BA & 0.576770 & 0.372420 & 0.026984 & $0.061 *$ \\
\hline H5BB & 0.607907 & 0.337836 & 0.108886 & $0.061^{*}$ \\
\hline H5BC & 0.510149 & 0.379233 & 0.069535 & $0.061 *$ \\
\hline C6B & $0.61290(18)$ & $0.1223(4)$ & $0.04453(18)$ & $0.0426(7)$ \\
\hline H6BA & 0.597246 & 0.031375 & 0.033235 & $0.064 *$ \\
\hline H6BB & 0.658496 & 0.127597 & 0.090215 & $0.064 *$ \\
\hline
\end{tabular}




\begin{tabular}{|c|c|c|c|c|}
\hline H6BC & 0.633488 & 0.159756 & 0.008298 & $0.064 *$ \\
\hline C7B & $0.38230(15)$ & $-0.0216(3)$ & $0.14727(13)$ & $0.0262(4)$ \\
\hline $\mathrm{C} 8 \mathrm{~B}$ & 0.39567 (17) & $-0.1480(3)$ & $0.13133(16)$ & $0.0331(5)$ \\
\hline H8B & 0.379285 & -0.172851 & 0.083366 & $0.040^{*}$ \\
\hline C9B & $0.4325(2)$ & $-0.2415(3)$ & $0.18302(19)$ & $0.0416(7)$ \\
\hline H9B & 0.440569 & -0.327656 & 0.169875 & $0.050 *$ \\
\hline $\mathrm{C} 10 \mathrm{~B}$ & $0.4565(2)$ & $-0.2081(3)$ & $0.25186(19)$ & $0.0447(8)$ \\
\hline H10B & 0.480564 & -0.271730 & 0.286887 & $0.054 *$ \\
\hline C11B & $0.44597(19)$ & $-0.0799(4)$ & $0.27190(15)$ & $0.0408(6)$ \\
\hline C12B & $0.4749(3)$ & $-0.0458(5)$ & $0.34383(18)$ & $0.0598(11)$ \\
\hline H12B & 0.499686 & -0.110313 & 0.378138 & $0.072^{*}$ \\
\hline C13B & 0.4677 (3) & $0.0782(6)$ & $0.36478(18)$ & $0.0675(12)$ \\
\hline H13B & 0.488485 & 0.100209 & 0.413208 & $0.081^{*}$ \\
\hline C14B & $0.4294(3)$ & $0.1732(4)$ & $0.31411(18)$ & $0.0557(9)$ \\
\hline H14B & 0.423864 & 0.259353 & 0.328671 & $0.067^{*}$ \\
\hline C15B & $0.3998(2)$ & $0.1431(3)$ & $0.24405(15)$ & $0.0378(6)$ \\
\hline H15B & 0.373284 & 0.208679 & 0.210960 & $0.045^{*}$ \\
\hline C16B & $0.40786(17)$ & $0.0161(3)$ & $0.21970(14)$ & $0.0312(5)$ \\
\hline $\mathrm{C} 17 \mathrm{~B}$ & $0.18258(14)$ & $0.0260(2)$ & $0.07173(12)$ & $0.0240(4)$ \\
\hline $\mathrm{C} 18 \mathrm{~B}$ & $0.17658(17)$ & $-0.0904(3)$ & $0.03616(16)$ & $0.0362(6)$ \\
\hline H18B & 0.222145 & -0.116942 & 0.020048 & $0.043 *$ \\
\hline C19B & $0.10414(19)$ & $-0.1690(3)$ & $0.02384(17)$ & $0.0403(6)$ \\
\hline H19B & 0.101668 & -0.249542 & 0.000471 & $0.048^{*}$ \\
\hline $\mathrm{C} 20 \mathrm{~B}$ & $0.03663(18)$ & $-0.1320(3)$ & $0.04485(16)$ & $0.0375(6)$ \\
\hline $\mathrm{H} 20 \mathrm{~B}$ & -0.013176 & -0.184875 & 0.035055 & $0.045^{*}$ \\
\hline $\mathrm{C} 21 \mathrm{~B}$ & $0.0420(2)$ & $-0.0165(4)$ & $0.0805(2)$ & $0.0458(7)$ \\
\hline $\mathrm{H} 21 \mathrm{~B}$ & -0.004282 & 0.010201 & 0.095651 & $0.055^{*}$ \\
\hline $\mathrm{C} 22 \mathrm{~B}$ & $0.11428(19)$ & $0.0607(3)$ & $0.09440(17)$ & $0.0368(6)$ \\
\hline $\mathrm{H} 22 \mathrm{~B}$ & 0.117436 & 0.138935 & 0.119927 & $0.044^{*}$ \\
\hline C23B & $0.22633(14)$ & $0.2390(2)$ & $0.03590(13)$ & $0.0239(4)$ \\
\hline $\mathrm{C} 24 \mathrm{~B}$ & $0.18545(14)$ & $0.2252(2)$ & $-0.03469(12)$ & $0.0231(4)$ \\
\hline H24B & 0.170904 & 0.141171 & -0.054186 & $0.028^{*}$ \\
\hline C25B & 0.16467 (14) & $0.3353(2)$ & $-0.07890(13)$ & $0.0245(4)$ \\
\hline $\mathrm{C} 26 \mathrm{~B}$ & $0.12002(16)$ & $0.3252(3)$ & $-0.15184(14)$ & $0.0270(5)$ \\
\hline H26B & 0.102173 & 0.243203 & -0.173015 & $0.032 *$ \\
\hline C27B & $0.10314(16)$ & $0.4359(3)$ & $-0.19113(13)$ & $0.0305(5)$ \\
\hline C28B & $0.13093(17)$ & $0.5590(3)$ & $-0.16304(16)$ & $0.0325(5)$ \\
\hline $\mathrm{H} 28 \mathrm{~B}$ & 0.120889 & 0.633205 & -0.192502 & $0.039 *$ \\
\hline C29B & $0.17314(16)$ & $0.5698(3)$ & $-0.09175(16)$ & $0.0321(5)$ \\
\hline $\mathrm{H} 29 \mathrm{~B}$ & 0.191820 & 0.652478 & -0.071822 & $0.039 *$ \\
\hline $\mathrm{C} 30 \mathrm{~B}$ & $0.18881(14)$ & $0.4591(2)$ & $-0.04815(14)$ & $0.0270(5)$ \\
\hline C31B & $0.24184(15)$ & $0.3705(3)$ & $0.06140(14)$ & $0.0284(5)$ \\
\hline $\mathrm{C} 32 \mathrm{~B}$ & $0.2775(3)$ & $0.5082(4)$ & $0.1616(2)$ & $0.0605(11)$ \\
\hline $\mathrm{H} 32 \mathrm{D}$ & 0.312393 & 0.565497 & 0.143091 & $0.091 *$ \\
\hline $\mathrm{H} 32 \mathrm{E}$ & 0.302792 & 0.503171 & 0.212812 & $0.091 *$ \\
\hline $\mathrm{H} 32 \mathrm{~F}$ & 0.218838 & 0.542620 & 0.149019 & $0.091 *$ \\
\hline C33B & $0.64439(16)$ & $0.7125(3)$ & $0.11789(12)$ & $0.0275(5)$ \\
\hline C34B & $0.68382(16)$ & $0.7862(3)$ & $0.18508(12)$ & $0.0262(4)$ \\
\hline
\end{tabular}




\begin{tabular}{lllll} 
H34B & 0.740262 & 0.764391 & 0.214460 & $0.039(9)^{*}$ \\
C35B & $0.64195(16)$ & $0.8809(3)$ & $0.20449(13)$ & $0.0270(5)$ \\
H35B & 0.583327 & 0.894093 & 0.177285 & $0.036(9)^{*}$ \\
C36B & $0.67970(16)$ & $0.9671(3)$ & $0.26520(14)$ & $0.0294(5)$ \\
\hline
\end{tabular}

Atomic displacement parameters $\left(\AA^{2}\right)$

\begin{tabular}{|c|c|c|c|c|c|c|}
\hline & $U^{11}$ & $U^{22}$ & $U^{\beta 3}$ & $U^{12}$ & $U^{13}$ & $U^{23}$ \\
\hline Br1A & $0.0876(3)$ & 0.0599 (2) & $0.0575(2)$ & $0.0371(2)$ & $0.0360(2)$ & 0.02961 (19) \\
\hline O1A & $0.0325(9)$ & $0.0275(9)$ & $0.0287(8)$ & $-0.0026(7)$ & $0.0171(7)$ & $-0.0036(7)$ \\
\hline $\mathrm{O} 2 \mathrm{~A}$ & $0.0461(12)$ & $0.0492(14)$ & $0.0444(12)$ & $0.0007(10)$ & $0.0073(10)$ & $-0.0244(11)$ \\
\hline $\mathrm{O} 3 \mathrm{~A}$ & $0.0314(9)$ & $0.0409(11)$ & $0.0239(8)$ & $-0.0039(8)$ & $-0.0006(7)$ & $0.0011(8)$ \\
\hline $\mathrm{O} 4 \mathrm{~A}$ & $0.0513(12)$ & $0.0284(9)$ & $0.0209(8)$ & $-0.0064(8)$ & $0.0065(8)$ & $-0.0016(7)$ \\
\hline $\mathrm{O} 5 \mathrm{~A}$ & $0.0397(10)$ & $0.0264(9)$ & 0.0319 (9) & $-0.0017(8)$ & $0.0024(8)$ & $0.0014(7)$ \\
\hline O6A & $0.0427(11)$ & $0.0284(9)$ & $0.0300(9)$ & $0.0029(8)$ & $-0.0027(8)$ & $-0.0046(7)$ \\
\hline N1A & $0.0175(8)$ & $0.0260(9)$ & $0.0287(9)$ & $-0.0018(7)$ & $0.0061(7)$ & 0.0007 (8) \\
\hline N2A & $0.0254(10)$ & $0.0290(11)$ & $0.0671(17)$ & $-0.0033(9)$ & $0.0154(11)$ & $-0.0140(12)$ \\
\hline $\mathrm{C} 1 \mathrm{~A}$ & $0.0206(10)$ & $0.0325(12)$ & $0.0239(10)$ & $0.0019(9)$ & $0.0089(8)$ & $-0.0032(9)$ \\
\hline $\mathrm{C} 2 \mathrm{~A}$ & $0.0199(9)$ & $0.0269(11)$ & $0.0270(11)$ & $0.0006(8)$ & $0.0104(8)$ & $-0.0003(8)$ \\
\hline $\mathrm{C} 3 \mathrm{~A}$ & $0.0206(10)$ & $0.0269(11)$ & $0.0309(11)$ & $-0.0004(8)$ & $0.0082(9)$ & $0.0004(9)$ \\
\hline $\mathrm{C} 4 \mathrm{~A}$ & $0.0233(11)$ & $0.0325(13)$ & $0.0417(14)$ & $0.0030(9)$ & $0.0150(10)$ & $0.0121(11)$ \\
\hline C5A & $0.0407(15)$ & $0.0458(17)$ & $0.0348(14)$ & $-0.0224(13)$ & $0.0125(12)$ & $-0.0103(12)$ \\
\hline C6A & $0.0382(15)$ & $0.0335(14)$ & $0.071(2)$ & 0.0077 (12) & $0.0326(15)$ & $0.0071(15)$ \\
\hline C7A & $0.0209(10)$ & $0.0325(13)$ & $0.0342(12)$ & $0.0000(9)$ & $0.0129(9)$ & $0.0030(9)$ \\
\hline C8A & $0.0437(15)$ & $0.0316(13)$ & $0.0409(15)$ & 0.0007 (11) & $0.0237(12)$ & $0.0043(11)$ \\
\hline C9A & $0.060(2)$ & $0.0337(15)$ & $0.060(2)$ & $0.0086(14)$ & $0.0344(17)$ & $0.0150(14)$ \\
\hline C10A & $0.0437(17)$ & 0.0495 (19) & $0.056(2)$ & $0.0096(14)$ & $0.0227(15)$ & $0.0258(16)$ \\
\hline $\mathrm{C} 11 \mathrm{~A}$ & $0.0295(12)$ & $0.0538(19)$ & $0.0378(14)$ & $-0.0025(12)$ & 0.0098 (11) & $0.0151(13)$ \\
\hline $\mathrm{C} 12 \mathrm{~A}$ & $0.0464(17)$ & $0.072(2)$ & $0.0335(14)$ & $-0.0056(18)$ & $0.0047(13)$ & $0.0172(17)$ \\
\hline $\mathrm{C} 13 \mathrm{~A}$ & $0.054(2)$ & $0.087(3)$ & $0.0293(15)$ & $-0.015(2)$ & $0.0020(14)$ & 0.0067 (17) \\
\hline C14A & $0.0524(18)$ & $0.059(2)$ & $0.0281(13)$ & $-0.0158(16)$ & $0.0079(13)$ & $-0.0067(13)$ \\
\hline C15A & $0.0344(13)$ & $0.0455(16)$ & $0.0257(12)$ & $-0.0101(12)$ & $0.0080(10)$ & $-0.0030(11)$ \\
\hline C16A & $0.0227(10)$ & $0.0420(14)$ & $0.0291(11)$ & $-0.0035(10)$ & $0.0072(9)$ & $0.0052(11)$ \\
\hline $\mathrm{C} 17 \mathrm{~A}$ & $0.0212(10)$ & $0.0394(14)$ & $0.0241(10)$ & $0.0016(9)$ & $0.0095(8)$ & $0.0059(9)$ \\
\hline C18A & $0.0367(15)$ & $0.0452(18)$ & $0.082(3)$ & 0.0018 (13) & $0.0390(17)$ & $-0.0005(17)$ \\
\hline C19A & $0.0343(15)$ & $0.062(2)$ & 0.087 (3) & $0.0035(15)$ & $0.0370(18)$ & $0.012(2)$ \\
\hline $\mathrm{C} 20 \mathrm{~A}$ & $0.0255(12)$ & $0.066(2)$ & 0.0507 (17) & $-0.0085(13)$ & $0.0119(12)$ & $0.0184(16)$ \\
\hline $\mathrm{C} 21 \mathrm{~A}$ & $0.0395(15)$ & $0.068(2)$ & $0.0419(16)$ & $-0.0241(16)$ & 0.0185 (13) & $-0.0132(16)$ \\
\hline $\mathrm{C} 22 \mathrm{~A}$ & $0.0339(13)$ & $0.064(2)$ & $0.0487(16)$ & $-0.0199(14)$ & $0.0249(12)$ & $-0.0228(16)$ \\
\hline $\mathrm{C} 23 \mathrm{~A}$ & $0.0212(10)$ & $0.0268(11)$ & $0.0352(12)$ & $0.0016(9)$ & $0.0116(9)$ & $-0.0069(9)$ \\
\hline $\mathrm{C} 24 \mathrm{~A}$ & $0.0231(10)$ & $0.0244(11)$ & $0.0346(12)$ & $0.0041(8)$ & $0.0132(9)$ & $-0.0011(9)$ \\
\hline $\mathrm{C} 25 \mathrm{~A}$ & $0.0225(10)$ & $0.0277(12)$ & $0.0462(14)$ & $0.0036(9)$ & $0.0171(10)$ & $0.0020(11)$ \\
\hline C26A & $0.0356(13)$ & $0.0339(14)$ & $0.0445(15)$ & $0.0123(11)$ & $0.0211(12)$ & $0.0072(12)$ \\
\hline C27A & $0.0404(15)$ & $0.0391(15)$ & $0.0610(19)$ & $0.0167(13)$ & $0.0274(14)$ & $0.0155(15)$ \\
\hline $\mathrm{C} 28 \mathrm{~A}$ & $0.0340(14)$ & $0.0332(15)$ & $0.081(3)$ & $0.0102(12)$ & $0.0274(16)$ & $0.0152(16)$ \\
\hline C29A & $0.0277(13)$ & $0.0251(13)$ & 0.087 (3) & $0.0011(10)$ & $0.0228(15)$ & $0.0017(15)$ \\
\hline C30A & $0.0206(10)$ & $0.0264(11)$ & $0.0600(17)$ & $0.0009(9)$ & $0.0167(11)$ & $-0.0022(12)$ \\
\hline C31A & $0.0233(11)$ & $0.0355(14)$ & $0.0487(16)$ & $-0.0014(10)$ & 0.0108 (11) & $-0.0159(12)$ \\
\hline
\end{tabular}




\begin{tabular}{|c|c|c|c|c|c|c|}
\hline C32A & $0.064(2)$ & $0.060(2)$ & $0.073(3)$ & $-0.016(2)$ & $0.029(2)$ & $-0.044(2)$ \\
\hline C33A & $0.0292(11)$ & $0.0269(11)$ & $0.0209(10)$ & $0.0030(9)$ & $0.0053(8)$ & $-0.0026(8)$ \\
\hline C34A & $0.0243(10)$ & $0.0285(11)$ & $0.0234(10)$ & $0.0001(9)$ & $0.0025(8)$ & $-0.0008(9)$ \\
\hline C35A & $0.0249(10)$ & $0.0270(11)$ & $0.0244(10)$ & $0.0002(9)$ & $0.0020(8)$ & $-0.0009(9)$ \\
\hline C36A & $0.0286(11)$ & $0.0251(12)$ & $0.0275(11)$ & $0.0021(9)$ & $0.0057(9)$ & $-0.0014(8)$ \\
\hline $\mathrm{Br} 1 \mathrm{~B}$ & $0.0713(2)$ & $0.03731(15)$ & $0.03003(13)$ & $0.00847(15)$ & 0.01267 (13) & $0.00635(12)$ \\
\hline O1B & $0.0293(8)$ & $0.0296(9)$ & $0.0256(8)$ & $0.0064(7)$ & $0.0151(7)$ & $0.0038(7)$ \\
\hline $\mathrm{O} 2 \mathrm{~B}$ & $0.0409(10)$ & $0.0350(10)$ & $0.0348(10)$ & $0.0019(8)$ & $0.0031(8)$ & $-0.0121(8)$ \\
\hline O3B & $0.0293(10)$ & $0.0694(16)$ & $0.0297(10)$ & $0.0111(10)$ & $-0.0034(8)$ & $-0.0150(10)$ \\
\hline O4B & $0.0356(9)$ & $0.0289(9)$ & $0.0230(8)$ & $0.0020(7)$ & $0.0084(7)$ & $-0.0004(7)$ \\
\hline O5B & $0.0342(10)$ & $0.0326(11)$ & $0.0631(14)$ & $0.0041(8)$ & $0.0079(10)$ & $-0.0164(10)$ \\
\hline O6B & 0.0472 (11) & $0.0280(9)$ & $0.0306(9)$ & $0.0058(9)$ & $-0.0054(8)$ & $-0.0075(9)$ \\
\hline N1B & $0.0164(8)$ & $0.0402(12)$ & $0.0255(9)$ & $-0.0009(8)$ & $0.0031(7)$ & $0.0079(9)$ \\
\hline $\mathrm{N} 2 \mathrm{~B}$ & $0.0229(9)$ & $0.0247(9)$ & $0.0402(12)$ & $-0.0008(8)$ & $0.0063(8)$ & $-0.0054(9)$ \\
\hline $\mathrm{C} 1 \mathrm{~B}$ & $0.0193(9)$ & $0.0272(11)$ & $0.0233(10)$ & $0.0022(8)$ & $0.0083(8)$ & $0.0006(8)$ \\
\hline $\mathrm{C} 2 \mathrm{~B}$ & $0.0193(9)$ & 0.0284 (11) & $0.0221(10)$ & $0.0043(8)$ & $0.0081(8)$ & $0.0057(8)$ \\
\hline C3B & $0.0196(10)$ & $0.0290(12)$ & $0.0322(11)$ & $0.0016(8)$ & $0.0095(9)$ & $0.0081(9)$ \\
\hline C4B & $0.0201(10)$ & $0.0364(13)$ & $0.0319(12)$ & $0.0031(9)$ & $0.0089(9)$ & $0.0121(10)$ \\
\hline C5B & $0.0310(13)$ & $0.0404(15)$ & $0.0454(16)$ & $-0.0101(11)$ & $0.0064(12)$ & $0.0031(13)$ \\
\hline C6B & $0.0250(12)$ & $0.059(2)$ & $0.0458(16)$ & 0.0085 (12) & $0.0149(11)$ & $0.0128(15)$ \\
\hline C7B & $0.0194(9)$ & $0.0311(12)$ & $0.0288(11)$ & $0.0012(9)$ & $0.0088(8)$ & $0.0057(10)$ \\
\hline $\mathrm{C} 8 \mathrm{~B}$ & $0.0300(12)$ & $0.0300(13)$ & $0.0422(14)$ & $0.0041(10)$ & $0.0160(11)$ & $0.0092(11)$ \\
\hline C9B & $0.0358(14)$ & $0.0357(15)$ & $0.0560(18)$ & $0.0083(12)$ & $0.0188(13)$ & $0.0171(13)$ \\
\hline $\mathrm{C} 10 \mathrm{~B}$ & $0.0351(14)$ & $0.0460(18)$ & $0.0516(18)$ & $0.0116(13)$ & $0.0121(13)$ & $0.0282(15)$ \\
\hline C11B & $0.0343(13)$ & $0.0529(17)$ & $0.0312(12)$ & $-0.0013(13)$ & $0.0055(10)$ & $0.0153(14)$ \\
\hline C12B & $0.060(2)$ & $0.078(3)$ & 0.0307 (14) & $0.004(2)$ & $0.0002(14)$ & $0.0205(17)$ \\
\hline C13B & $0.077(3)$ & $0.088(3)$ & $0.0234(14)$ & $-0.006(2)$ & $-0.0024(16)$ & $0.0039(17)$ \\
\hline C14B & $0.067(2)$ & $0.059(2)$ & $0.0308(15)$ & $-0.0105(18)$ & $0.0022(15)$ & $-0.0046(15)$ \\
\hline C15B & $0.0381(14)$ & $0.0442(16)$ & $0.0267(12)$ & $-0.0045(12)$ & $0.0047(11)$ & $0.0014(11)$ \\
\hline $\mathrm{C} 16 \mathrm{~B}$ & $0.0236(11)$ & $0.0400(14)$ & $0.0275(11)$ & $-0.0009(10)$ & $0.0052(9)$ & $0.0083(10)$ \\
\hline C17B & $0.0207(10)$ & $0.0295(11)$ & $0.0225(10)$ & $0.0029(8)$ & $0.0081(8)$ & $0.0042(8)$ \\
\hline C18B & $0.0282(11)$ & $0.0403(15)$ & $0.0452(14)$ & $-0.0070(11)$ & $0.0189(11)$ & $-0.0112(12)$ \\
\hline C19B & $0.0354(14)$ & $0.0434(16)$ & $0.0445(16)$ & $-0.0130(12)$ & $0.0163(12)$ & $-0.0110(13)$ \\
\hline $\mathrm{C} 20 \mathrm{~B}$ & $0.0278(12)$ & $0.0474(17)$ & $0.0382(14)$ & $-0.0103(11)$ & $0.0120(11)$ & $0.0046(13)$ \\
\hline $\mathrm{C} 21 \mathrm{~B}$ & 0.0317 (13) & $0.0533(19)$ & $0.063(2)$ & $-0.0026(13)$ & $0.0302(14)$ & $0.0012(17)$ \\
\hline $\mathrm{C} 22 \mathrm{~B}$ & $0.0330(13)$ & $0.0365(14)$ & $0.0499(16)$ & $-0.0005(11)$ & $0.0259(12)$ & $-0.0019(12)$ \\
\hline $\mathrm{C} 23 \mathrm{~B}$ & $0.0187(9)$ & $0.0231(10)$ & $0.0309(11)$ & $0.0022(8)$ & $0.0093(8)$ & $-0.0017(8)$ \\
\hline $\mathrm{C} 24 \mathrm{~B}$ & $0.0193(9)$ & $0.0220(10)$ & $0.0277(10)$ & $0.0029(8)$ & $0.0074(8)$ & $-0.0002(8)$ \\
\hline $\mathrm{C} 25 \mathrm{~B}$ & $0.0188(9)$ & $0.0242(11)$ & $0.0309(11)$ & $0.0030(8)$ & $0.0088(8)$ & $0.0014(9)$ \\
\hline $\mathrm{C} 26 \mathrm{~B}$ & $0.0273(11)$ & $0.0247(11)$ & $0.0313(12)$ & $0.0028(9)$ & $0.0127(9)$ & $0.0021(9)$ \\
\hline C27B & $0.0309(11)$ & $0.0313(13)$ & $0.0317(11)$ & $0.0055(10)$ & $0.0136(9)$ & $0.0061(10)$ \\
\hline C28B & $0.0293(12)$ & $0.0269(12)$ & $0.0454(15)$ & $0.0029(10)$ & $0.0180(11)$ & $0.0089(11)$ \\
\hline C29B & $0.0233(11)$ & $0.0232(11)$ & $0.0519(16)$ & $-0.0007(9)$ & $0.0151(11)$ & $0.0020(11)$ \\
\hline $\mathrm{C} 30 \mathrm{~B}$ & $0.0177(9)$ & $0.0224(11)$ & $0.0410(13)$ & $0.0008(8)$ & $0.0096(9)$ & $0.0008(9)$ \\
\hline C31B & $0.0203(10)$ & $0.0284(12)$ & $0.0345(12)$ & $0.0007(9)$ & $0.0063(9)$ & $-0.0061(10)$ \\
\hline $\mathrm{C} 32 \mathrm{~B}$ & $0.072(3)$ & $0.044(2)$ & $0.051(2)$ & $-0.0037(17)$ & $0.0006(18)$ & $-0.0245(16)$ \\
\hline C33B & $0.0273(11)$ & $0.0343(13)$ & $0.0196(10)$ & $0.0008(9)$ & $0.0059(8)$ & $-0.0007(9)$ \\
\hline C34B & $0.0241(10)$ & $0.0299(12)$ & $0.0223(10)$ & $0.0010(9)$ & $0.0043(8)$ & $-0.0013(9)$ \\
\hline
\end{tabular}




\begin{tabular}{lllllll}
$\mathrm{C} 35 \mathrm{~B}$ & $0.0242(10)$ & $0.0263(11)$ & $0.0277(11)$ & $0.0007(9)$ & $0.0048(9)$ & $0.0004(9)$ \\
$\mathrm{C} 36 \mathrm{~B}$ & $0.0295(11)$ & $0.0245(11)$ & $0.0330(12)$ & $0.0004(9)$ & $0.0085(9)$ & $-0.0015(9)$ \\
\hline
\end{tabular}

Geometric parameters $\left(\AA,{ }^{\circ}\right)$

\begin{tabular}{|c|c|c|c|}
\hline $\mathrm{Br} 1 \mathrm{~A}-\mathrm{C} 27 \mathrm{~A}$ & $1.891(4)$ & $\mathrm{Br} 1 \mathrm{~B}-\mathrm{C} 27 \mathrm{~B}$ & $1.902(3)$ \\
\hline $\mathrm{O} 1 \mathrm{~A}-\mathrm{C} 2 \mathrm{~A}$ & $1.425(3)$ & $\mathrm{O} 1 \mathrm{~B}-\mathrm{C} 2 \mathrm{~B}$ & $1.425(3)$ \\
\hline $\mathrm{O} 1 \mathrm{~A}-\mathrm{H} 1 \mathrm{AB}$ & $0.86(4)$ & $\mathrm{O} 1 \mathrm{~B}-\mathrm{H} 1 \mathrm{~B}$ & $0.89(4)$ \\
\hline $\mathrm{O} 2 \mathrm{~A}-\mathrm{C} 31 \mathrm{~A}$ & $1.363(4)$ & $\mathrm{O} 2 \mathrm{~B}-\mathrm{C} 31 \mathrm{~B}$ & $1.354(3)$ \\
\hline $\mathrm{O} 2 \mathrm{~A}-\mathrm{C} 32 \mathrm{~A}$ & $1.434(4)$ & $\mathrm{O} 2 \mathrm{~B}-\mathrm{C} 32 \mathrm{~B}$ & $1.430(4)$ \\
\hline $\mathrm{O} 3 \mathrm{~A}-\mathrm{C} 33 \mathrm{~A}$ & $1.258(3)$ & $\mathrm{O} 3 \mathrm{~B}-\mathrm{C} 33 \mathrm{~B}$ & $1.261(3)$ \\
\hline $\mathrm{O} 4 \mathrm{~A}-\mathrm{C} 33 \mathrm{~A}$ & $1.263(3)$ & $\mathrm{O} 4 \mathrm{~B}-\mathrm{C} 33 \mathrm{~B}$ & $1.257(3)$ \\
\hline $\mathrm{O} 5 \mathrm{~A}-\mathrm{C} 36 \mathrm{~A}$ & $1.323(3)$ & $\mathrm{O} 5 \mathrm{~B}-\mathrm{C} 36 \mathrm{~B}$ & $1.211(3)$ \\
\hline $\mathrm{O} 5 \mathrm{~A}-\mathrm{H} 5 \mathrm{~A}$ & $1.04(4)$ & $\mathrm{O} 6 \mathrm{~B}-\mathrm{C} 36 \mathrm{~B}$ & $1.326(3)$ \\
\hline $\mathrm{O} 6 \mathrm{~A}-\mathrm{C} 36 \mathrm{~A}$ & $1.206(3)$ & O6B-H6B & $0.86(5)$ \\
\hline N1A-C5A & $1.478(3)$ & $\mathrm{N} 1 \mathrm{~B}-\mathrm{C} 6 \mathrm{~B}$ & $1.479(4)$ \\
\hline $\mathrm{N} 1 \mathrm{~A}-\mathrm{C} 6 \mathrm{~A}$ & $1.485(4)$ & $\mathrm{N} 1 \mathrm{~B}-\mathrm{C} 5 \mathrm{~B}$ & $1.484(4)$ \\
\hline $\mathrm{N} 1 \mathrm{~A}-\mathrm{C} 4 \mathrm{~A}$ & $1.498(3)$ & $\mathrm{N} 1 \mathrm{~B}-\mathrm{C} 4 \mathrm{~B}$ & $1.491(3)$ \\
\hline $\mathrm{N} 1 \mathrm{~A}-\mathrm{H} 1 \mathrm{AN}$ & $0.98(4)$ & $\mathrm{N} 1 \mathrm{~B}-\mathrm{H} 1 \mathrm{BN}$ & $0.85(4)$ \\
\hline $\mathrm{N} 2 \mathrm{~A}-\mathrm{C} 31 \mathrm{~A}$ & $1.296(4)$ & $\mathrm{N} 2 \mathrm{~B}-\mathrm{C} 31 \mathrm{~B}$ & $1.299(4)$ \\
\hline $\mathrm{N} 2 \mathrm{~A}-\mathrm{C} 30 \mathrm{~A}$ & $1.371(4)$ & $\mathrm{N} 2 \mathrm{~B}-\mathrm{C} 30 \mathrm{~B}$ & $1.372(4)$ \\
\hline $\mathrm{C} 1 \mathrm{~A}-\mathrm{C} 23 \mathrm{~A}$ & $1.514(4)$ & $\mathrm{C} 1 \mathrm{~B}-\mathrm{C} 23 \mathrm{~B}$ & $1.511(3)$ \\
\hline $\mathrm{C} 1 \mathrm{~A}-\mathrm{C} 17 \mathrm{~A}$ & $1.535(3)$ & $\mathrm{C} 1 \mathrm{~B}-\mathrm{C} 17 \mathrm{~B}$ & $1.536(3)$ \\
\hline $\mathrm{C} 1 \mathrm{~A}-\mathrm{C} 2 \mathrm{~A}$ & $1.568(3)$ & $\mathrm{C} 1 \mathrm{~B}-\mathrm{C} 2 \mathrm{~B}$ & $1.576(3)$ \\
\hline $\mathrm{C} 1 \mathrm{~A}-\mathrm{H} 1 \mathrm{~A}$ & 1.0000 & $\mathrm{C} 1 \mathrm{~B}-\mathrm{H} 1 \mathrm{BA}$ & 1.0000 \\
\hline $\mathrm{C} 2 \mathrm{~A}-\mathrm{C} 7 \mathrm{~A}$ & $1.535(4)$ & $\mathrm{C} 2 \mathrm{~B}-\mathrm{C} 7 \mathrm{~B}$ & $1.539(3)$ \\
\hline $\mathrm{C} 2 \mathrm{~A}-\mathrm{C} 3 \mathrm{~A}$ & $1.551(3)$ & $\mathrm{C} 2 \mathrm{~B}-\mathrm{C} 3 \mathrm{~B}$ & $1.548(3)$ \\
\hline $\mathrm{C} 3 \mathrm{~A}-\mathrm{C} 4 \mathrm{~A}$ & $1.535(3)$ & $\mathrm{C} 3 \mathrm{~B}-\mathrm{C} 4 \mathrm{~B}$ & $1.530(3)$ \\
\hline $\mathrm{C} 3 \mathrm{~A}-\mathrm{H} 3 \mathrm{AA}$ & 0.9900 & $\mathrm{C} 3 \mathrm{~B}-\mathrm{H} 3 \mathrm{BA}$ & 0.9900 \\
\hline $\mathrm{C} 3 \mathrm{~A}-\mathrm{H} 3 \mathrm{AB}$ & 0.9900 & $\mathrm{C} 3 \mathrm{~B}-\mathrm{H} 3 \mathrm{BB}$ & 0.9900 \\
\hline $\mathrm{C} 4 \mathrm{~A}-\mathrm{H} 4 \mathrm{AA}$ & 0.9900 & $\mathrm{C} 4 \mathrm{~B}-\mathrm{H} 4 \mathrm{BA}$ & 0.9900 \\
\hline $\mathrm{C} 4 \mathrm{~A}-\mathrm{H} 4 \mathrm{AB}$ & 0.9900 & $\mathrm{C} 4 \mathrm{~B}-\mathrm{H} 4 \mathrm{BB}$ & 0.9900 \\
\hline $\mathrm{C} 5 \mathrm{~A}-\mathrm{H} 5 \mathrm{AA}$ & 0.9800 & $\mathrm{C} 5 \mathrm{~B}-\mathrm{H} 5 \mathrm{BA}$ & 0.9800 \\
\hline $\mathrm{C} 5 \mathrm{~A}-\mathrm{H} 5 \mathrm{AB}$ & 0.9800 & $\mathrm{C} 5 \mathrm{~B}-\mathrm{H} 5 \mathrm{BB}$ & 0.9800 \\
\hline $\mathrm{C} 5 \mathrm{~A}-\mathrm{H} 5 \mathrm{AC}$ & 0.9800 & $\mathrm{C} 5 \mathrm{~B}-\mathrm{H} 5 \mathrm{BC}$ & 0.9800 \\
\hline C6A-H6AA & 0.9800 & C6B-H6BA & 0.9800 \\
\hline C6A-H6AB & 0.9800 & C6B-H6BB & 0.9800 \\
\hline C6A-H6AC & 0.9800 & $\mathrm{C} 6 \mathrm{~B}-\mathrm{H} 6 \mathrm{BC}$ & 0.9800 \\
\hline $\mathrm{C} 7 \mathrm{~A}-\mathrm{C} 8 \mathrm{~A}$ & $1.381(4)$ & $\mathrm{C} 7 \mathrm{~B}-\mathrm{C} 8 \mathrm{~B}$ & $1.378(4)$ \\
\hline $\mathrm{C} 7 \mathrm{~A}-\mathrm{C} 16 \mathrm{~A}$ & $1.437(4)$ & $\mathrm{C} 7 \mathrm{~B}-\mathrm{C} 16 \mathrm{~B}$ & $1.434(4)$ \\
\hline $\mathrm{C} 8 \mathrm{~A}-\mathrm{C} 9 \mathrm{~A}$ & $1.406(4)$ & $\mathrm{C} 8 \mathrm{~B}-\mathrm{C} 9 \mathrm{~B}$ & $1.403(4)$ \\
\hline $\mathrm{C} 8 \mathrm{~A}-\mathrm{H} 8 \mathrm{~A}$ & 0.9500 & $\mathrm{C} 8 \mathrm{~B}-\mathrm{H} 8 \mathrm{~B}$ & 0.9500 \\
\hline $\mathrm{C} 9 \mathrm{~A}-\mathrm{C} 10 \mathrm{~A}$ & $1.355(6)$ & $\mathrm{C} 9 \mathrm{~B}-\mathrm{C} 10 \mathrm{~B}$ & $1.357(5)$ \\
\hline C9A-H9A & 0.9500 & C9B-H9B & 0.9500 \\
\hline $\mathrm{C} 10 \mathrm{~A}-\mathrm{C} 11 \mathrm{~A}$ & $1.417(6)$ & $\mathrm{C} 10 \mathrm{~B}-\mathrm{C} 11 \mathrm{~B}$ & $1.411(6)$ \\
\hline $\mathrm{C} 10 \mathrm{~A}-\mathrm{H} 10 \mathrm{~A}$ & 0.9500 & $\mathrm{C} 10 \mathrm{~B}-\mathrm{H} 10 \mathrm{~B}$ & 0.9500 \\
\hline $\mathrm{C} 11 \mathrm{~A}-\mathrm{C} 12 \mathrm{~A}$ & $1.416(5)$ & $\mathrm{C} 11 \mathrm{~B}-\mathrm{C} 12 \mathrm{~B}$ & $1.414(5)$ \\
\hline $\mathrm{C} 11 \mathrm{~A}-\mathrm{C} 16 \mathrm{~A}$ & $1.432(4)$ & $\mathrm{C} 11 \mathrm{~B}-\mathrm{C} 16 \mathrm{~B}$ & $1.432(4)$ \\
\hline
\end{tabular}




\begin{tabular}{|c|c|c|c|}
\hline $\mathrm{C} 12 \mathrm{~A}-\mathrm{C} 13 \mathrm{~A}$ & $1.351(7)$ & $\mathrm{C} 12 \mathrm{~B}-\mathrm{C} 13 \mathrm{~B}$ & $1.365(7)$ \\
\hline $\mathrm{C} 12 \mathrm{~A}-\mathrm{H} 12 \mathrm{~A}$ & 0.9500 & $\mathrm{C} 12 \mathrm{~B}-\mathrm{H} 12 \mathrm{~B}$ & 0.9500 \\
\hline $\mathrm{C} 13 \mathrm{~A}-\mathrm{C} 14 \mathrm{~A}$ & $1.406(6)$ & $\mathrm{C} 13 \mathrm{~B}-\mathrm{C} 14 \mathrm{~B}$ & $1.407(6)$ \\
\hline $\mathrm{C} 13 \mathrm{~A}-\mathrm{H} 13 \mathrm{~A}$ & 0.9500 & $\mathrm{C} 13 \mathrm{~B}-\mathrm{H} 13 \mathrm{~B}$ & 0.9500 \\
\hline $\mathrm{C} 14 \mathrm{~A}-\mathrm{C} 15 \mathrm{~A}$ & $1.369(4)$ & $\mathrm{C} 14 \mathrm{~B}-\mathrm{C} 15 \mathrm{~B}$ & $1.370(4)$ \\
\hline $\mathrm{C} 14 \mathrm{~A}-\mathrm{H} 14 \mathrm{~A}$ & 0.9500 & $\mathrm{C} 14 \mathrm{~B}-\mathrm{H} 14 \mathrm{~B}$ & 0.9500 \\
\hline $\mathrm{C} 15 \mathrm{~A}-\mathrm{C} 16 \mathrm{~A}$ & $1.430(5)$ & $\mathrm{C} 15 \mathrm{~B}-\mathrm{C} 16 \mathrm{~B}$ & $1.421(4)$ \\
\hline $\mathrm{C} 15 \mathrm{~A}-\mathrm{H} 15 \mathrm{~A}$ & 0.9500 & $\mathrm{C} 15 \mathrm{~B}-\mathrm{H} 15 \mathrm{~B}$ & 0.9500 \\
\hline $\mathrm{C} 17 \mathrm{~A}-\mathrm{C} 22 \mathrm{~A}$ & $1.371(4)$ & $\mathrm{C} 17 \mathrm{~B}-\mathrm{C} 18 \mathrm{~B}$ & $1.387(4)$ \\
\hline $\mathrm{C} 17 \mathrm{~A}-\mathrm{C} 18 \mathrm{~A}$ & $1.387(4)$ & $\mathrm{C} 17 \mathrm{~B}-\mathrm{C} 22 \mathrm{~B}$ & $1.392(3)$ \\
\hline $\mathrm{C} 18 \mathrm{~A}-\mathrm{C} 19 \mathrm{~A}$ & $1.391(5)$ & $\mathrm{C} 18 \mathrm{~B}-\mathrm{C} 19 \mathrm{~B}$ & $1.395(4)$ \\
\hline $\mathrm{C} 18 \mathrm{~A}-\mathrm{H} 18 \mathrm{~A}$ & 0.9500 & $\mathrm{C} 18 \mathrm{~B}-\mathrm{H} 18 \mathrm{~B}$ & 0.9500 \\
\hline $\mathrm{C} 19 \mathrm{~A}-\mathrm{C} 20 \mathrm{~A}$ & $1.357(6)$ & $\mathrm{C} 19 \mathrm{~B}-\mathrm{C} 20 \mathrm{~B}$ & $1.367(4)$ \\
\hline C19A-H19A & 0.9500 & $\mathrm{C} 19 \mathrm{~B}-\mathrm{H} 19 \mathrm{~B}$ & 0.9500 \\
\hline $\mathrm{C} 20 \mathrm{~A}-\mathrm{C} 21 \mathrm{~A}$ & $1.357(5)$ & $\mathrm{C} 20 \mathrm{~B}-\mathrm{C} 21 \mathrm{~B}$ & $1.380(5)$ \\
\hline $\mathrm{C} 20 \mathrm{~A}-\mathrm{H} 20 \mathrm{~A}$ & 0.9500 & $\mathrm{C} 20 \mathrm{~B}-\mathrm{H} 20 \mathrm{~B}$ & 0.9500 \\
\hline $\mathrm{C} 21 \mathrm{~A}-\mathrm{C} 22 \mathrm{~A}$ & $1.403(4)$ & $\mathrm{C} 21 \mathrm{~B}-\mathrm{C} 22 \mathrm{~B}$ & $1.381(4)$ \\
\hline $\mathrm{C} 21 \mathrm{~A}-\mathrm{H} 21 \mathrm{~A}$ & 0.9500 & $\mathrm{C} 21 \mathrm{~B}-\mathrm{H} 21 \mathrm{~B}$ & 0.9500 \\
\hline $\mathrm{C} 22 \mathrm{~A}-\mathrm{H} 22 \mathrm{~A}$ & 0.9500 & $\mathrm{C} 22 \mathrm{~B}-\mathrm{H} 22 \mathrm{~B}$ & 0.9500 \\
\hline $\mathrm{C} 23 \mathrm{~A}-\mathrm{C} 24 \mathrm{~A}$ & $1.366(4)$ & $\mathrm{C} 23 \mathrm{~B}-\mathrm{C} 24 \mathrm{~B}$ & $1.367(3)$ \\
\hline $\mathrm{C} 23 \mathrm{~A}-\mathrm{C} 31 \mathrm{~A}$ & $1.443(4)$ & $\mathrm{C} 23 \mathrm{~B}-\mathrm{C} 31 \mathrm{~B}$ & $1.444(3)$ \\
\hline $\mathrm{C} 24 \mathrm{~A}-\mathrm{C} 25 \mathrm{~A}$ & $1.415(4)$ & $\mathrm{C} 24 \mathrm{~B}-\mathrm{C} 25 \mathrm{~B}$ & $1.415(3)$ \\
\hline $\mathrm{C} 24 \mathrm{~A}-\mathrm{H} 24 \mathrm{~A}$ & 0.9500 & $\mathrm{C} 24 \mathrm{~B}-\mathrm{H} 24 \mathrm{~B}$ & 0.9500 \\
\hline $\mathrm{C} 25 \mathrm{~A}-\mathrm{C} 30 \mathrm{~A}$ & $1.413(4)$ & $\mathrm{C} 25 \mathrm{~B}-\mathrm{C} 26 \mathrm{~B}$ & $1.415(4)$ \\
\hline $\mathrm{C} 25 \mathrm{~A}-\mathrm{C} 26 \mathrm{~A}$ & $1.414(4)$ & $\mathrm{C} 25 \mathrm{~B}-\mathrm{C} 30 \mathrm{~B}$ & $1.419(4)$ \\
\hline $\mathrm{C} 26 \mathrm{~A}-\mathrm{C} 27 \mathrm{~A}$ & $1.370(4)$ & $\mathrm{C} 26 \mathrm{~B}-\mathrm{C} 27 \mathrm{~B}$ & $1.366(4)$ \\
\hline $\mathrm{C} 26 \mathrm{~A}-\mathrm{H} 26 \mathrm{~A}$ & 0.9500 & $\mathrm{C} 26 \mathrm{~B}-\mathrm{H} 26 \mathrm{~B}$ & 0.9500 \\
\hline $\mathrm{C} 27 \mathrm{~A}-\mathrm{C} 28 \mathrm{~A}$ & $1.397(5)$ & $\mathrm{C} 27 \mathrm{~B}-\mathrm{C} 28 \mathrm{~B}$ & $1.404(4)$ \\
\hline $\mathrm{C} 28 \mathrm{~A}-\mathrm{C} 29 \mathrm{~A}$ & $1.366(6)$ & $\mathrm{C} 28 \mathrm{~B}-\mathrm{C} 29 \mathrm{~B}$ & $1.380(4)$ \\
\hline $\mathrm{C} 28 \mathrm{~A}-\mathrm{H} 28 \mathrm{~A}$ & 0.9500 & $\mathrm{C} 28 \mathrm{~B}-\mathrm{H} 28 \mathrm{~B}$ & 0.9500 \\
\hline $\mathrm{C} 29 \mathrm{~A}-\mathrm{C} 30 \mathrm{~A}$ & $1.417(5)$ & $\mathrm{C} 29 \mathrm{~B}-\mathrm{C} 30 \mathrm{~B}$ & $1.413(4)$ \\
\hline $\mathrm{C} 29 \mathrm{~A}-\mathrm{H} 29 \mathrm{~A}$ & 0.9500 & $\mathrm{C} 29 \mathrm{~B}-\mathrm{H} 29 \mathrm{~B}$ & 0.9500 \\
\hline $\mathrm{C} 32 \mathrm{~A}-\mathrm{H} 32 \mathrm{~A}$ & 0.9800 & $\mathrm{C} 32 \mathrm{~B}-\mathrm{H} 32 \mathrm{D}$ & 0.9800 \\
\hline $\mathrm{C} 32 \mathrm{~A}-\mathrm{H} 32 \mathrm{~B}$ & 0.9800 & $\mathrm{C} 32 \mathrm{~B}-\mathrm{H} 32 \mathrm{E}$ & 0.9800 \\
\hline $\mathrm{C} 32 \mathrm{~A}-\mathrm{H} 32 \mathrm{C}$ & 0.9800 & $\mathrm{C} 32 \mathrm{~B}-\mathrm{H} 32 \mathrm{~F}$ & 0.9800 \\
\hline $\mathrm{C} 33 \mathrm{~A}-\mathrm{C} 34 \mathrm{~A}$ & $1.498(3)$ & $\mathrm{C} 33 \mathrm{~B}-\mathrm{C} 34 \mathrm{~B}$ & $1.502(3)$ \\
\hline $\mathrm{C} 34 \mathrm{~A}-\mathrm{C} 35 \mathrm{~A}$ & $1.320(4)$ & $\mathrm{C} 34 \mathrm{~B}-\mathrm{C} 35 \mathrm{~B}$ & $1.327(4)$ \\
\hline $\mathrm{C} 34 \mathrm{~A}-\mathrm{H} 34 \mathrm{~A}$ & 0.9500 & $\mathrm{C} 34 \mathrm{~B}-\mathrm{H} 34 \mathrm{~B}$ & 0.9500 \\
\hline $\mathrm{C} 35 \mathrm{~A}-\mathrm{C} 36 \mathrm{~A}$ & $1.498(3)$ & $\mathrm{C} 35 \mathrm{~B}-\mathrm{C} 36 \mathrm{~B}$ & $1.476(4)$ \\
\hline $\mathrm{C} 35 \mathrm{~A}-\mathrm{H} 35 \mathrm{~A}$ & 0.9500 & $\mathrm{C} 35 \mathrm{~B}-\mathrm{H} 35 \mathrm{~B}$ & 0.9500 \\
\hline $\mathrm{C} 2 \mathrm{~A}-\mathrm{O} 1 \mathrm{~A}-\mathrm{H} 1 \mathrm{AB}$ & $112(3)$ & $\mathrm{C} 2 \mathrm{~B}-\mathrm{O} 1 \mathrm{~B}-\mathrm{H} 1 \mathrm{~B}$ & $111(3)$ \\
\hline $\mathrm{C} 31 \mathrm{~A}-\mathrm{O} 2 \mathrm{~A}-\mathrm{C} 32 \mathrm{~A}$ & $117.6(3)$ & $\mathrm{C} 31 \mathrm{~B}-\mathrm{O} 2 \mathrm{~B}-\mathrm{C} 32 \mathrm{~B}$ & $117.4(3)$ \\
\hline $\mathrm{C} 36 \mathrm{~A}-\mathrm{O} 5 \mathrm{~A}-\mathrm{H} 5 \mathrm{~A}$ & $107(3)$ & $\mathrm{C} 36 \mathrm{~B}-\mathrm{O} 6 \mathrm{~B}-\mathrm{H} 6 \mathrm{~B}$ & $105(3)$ \\
\hline $\mathrm{C} 5 \mathrm{~A}-\mathrm{N} 1 \mathrm{~A}-\mathrm{C} 6 \mathrm{~A}$ & $110.7(2)$ & $\mathrm{C} 6 \mathrm{~B}-\mathrm{N} 1 \mathrm{~B}-\mathrm{C} 5 \mathrm{~B}$ & $109.8(2)$ \\
\hline $\mathrm{C} 5 \mathrm{~A}-\mathrm{N} 1 \mathrm{~A}-\mathrm{C} 4 \mathrm{~A}$ & $112.5(2)$ & $\mathrm{C} 6 \mathrm{~B}-\mathrm{N} 1 \mathrm{~B}-\mathrm{C} 4 \mathrm{~B}$ & $110.6(2)$ \\
\hline $\mathrm{C} 6 \mathrm{~A}-\mathrm{N} 1 \mathrm{~A}-\mathrm{C} 4 \mathrm{~A}$ & $110.8(2)$ & $\mathrm{C} 5 \mathrm{~B}-\mathrm{N} 1 \mathrm{~B}-\mathrm{C} 4 \mathrm{~B}$ & $113.6(2)$ \\
\hline $\mathrm{C} 5 \mathrm{~A}-\mathrm{N} 1 \mathrm{~A}-\mathrm{H} 1 \mathrm{AN}$ & $110(2)$ & $\mathrm{C} 6 \mathrm{~B}-\mathrm{N} 1 \mathrm{~B}-\mathrm{H} 1 \mathrm{BN}$ & $104(3)$ \\
\hline
\end{tabular}




\begin{tabular}{|c|c|c|c|}
\hline $\mathrm{C} 6 \mathrm{~A}-\mathrm{N} 1 \mathrm{~A}-\mathrm{H} 1 \mathrm{AN}$ & $104(2)$ & $\mathrm{C} 5 \mathrm{~B}-\mathrm{N} 1 \mathrm{~B}-\mathrm{H} 1 \mathrm{BN}$ & $109(3)$ \\
\hline $\mathrm{C} 4 \mathrm{~A}-\mathrm{N} 1 \mathrm{~A}-\mathrm{H} 1 \mathrm{AN}$ & $108(2)$ & $\mathrm{C} 4 \mathrm{~B}-\mathrm{N} 1 \mathrm{~B}-\mathrm{H} 1 \mathrm{BN}$ & $109(3)$ \\
\hline $\mathrm{C} 31 \mathrm{~A}-\mathrm{N} 2 \mathrm{~A}-\mathrm{C} 30 \mathrm{~A}$ & $117.5(3)$ & $\mathrm{C} 31 \mathrm{~B}-\mathrm{N} 2 \mathrm{~B}-\mathrm{C} 30 \mathrm{~B}$ & $117.4(2)$ \\
\hline $\mathrm{C} 23 \mathrm{~A}-\mathrm{C} 1 \mathrm{~A}-\mathrm{C} 17 \mathrm{~A}$ & $109.67(19)$ & $\mathrm{C} 23 \mathrm{~B}-\mathrm{C} 1 \mathrm{~B}-\mathrm{C} 17 \mathrm{~B}$ & $109.78(18)$ \\
\hline $\mathrm{C} 23 \mathrm{~A}-\mathrm{C} 1 \mathrm{~A}-\mathrm{C} 2 \mathrm{~A}$ & $111.83(19)$ & $\mathrm{C} 23 \mathrm{~B}-\mathrm{C} 1 \mathrm{~B}-\mathrm{C} 2 \mathrm{~B}$ & $111.04(18)$ \\
\hline $\mathrm{C} 17 \mathrm{~A}-\mathrm{C} 1 \mathrm{~A}-\mathrm{C} 2 \mathrm{~A}$ & $116.8(2)$ & $\mathrm{C} 17 \mathrm{~B}-\mathrm{C} 1 \mathrm{~B}-\mathrm{C} 2 \mathrm{~B}$ & $116.5(2)$ \\
\hline $\mathrm{C} 23 \mathrm{~A}-\mathrm{C} 1 \mathrm{~A}-\mathrm{H} 1 \mathrm{~A}$ & 105.9 & $\mathrm{C} 23 \mathrm{~B}-\mathrm{C} 1 \mathrm{~B}-\mathrm{H} 1 \mathrm{BA}$ & 106.3 \\
\hline $\mathrm{C} 17 \mathrm{~A}-\mathrm{C} 1 \mathrm{~A}-\mathrm{H} 1 \mathrm{~A}$ & 105.9 & $\mathrm{C} 17 \mathrm{~B}-\mathrm{C} 1 \mathrm{~B}-\mathrm{H} 1 \mathrm{BA}$ & 106.3 \\
\hline $\mathrm{C} 2 \mathrm{~A}-\mathrm{C} 1 \mathrm{~A}-\mathrm{H} 1 \mathrm{~A}$ & 105.9 & $\mathrm{C} 2 \mathrm{~B}-\mathrm{C} 1 \mathrm{~B}-\mathrm{H} 1 \mathrm{BA}$ & 106.3 \\
\hline $\mathrm{O} 1 \mathrm{~A}-\mathrm{C} 2 \mathrm{~A}-\mathrm{C} 7 \mathrm{~A}$ & $107.7(2)$ & $\mathrm{O} 1 \mathrm{~B}-\mathrm{C} 2 \mathrm{~B}-\mathrm{C} 7 \mathrm{~B}$ & $107.4(2)$ \\
\hline $\mathrm{O} 1 \mathrm{~A}-\mathrm{C} 2 \mathrm{~A}-\mathrm{C} 3 \mathrm{~A}$ & $106.58(19)$ & $\mathrm{O} 1 \mathrm{~B}-\mathrm{C} 2 \mathrm{~B}-\mathrm{C} 3 \mathrm{~B}$ & $106.19(18)$ \\
\hline $\mathrm{C} 7 \mathrm{~A}-\mathrm{C} 2 \mathrm{~A}-\mathrm{C} 3 \mathrm{~A}$ & $110.6(2)$ & $\mathrm{C} 7 \mathrm{~B}-\mathrm{C} 2 \mathrm{~B}-\mathrm{C} 3 \mathrm{~B}$ & $111.09(19)$ \\
\hline $\mathrm{O} 1 \mathrm{~A}-\mathrm{C} 2 \mathrm{~A}-\mathrm{C} 1 \mathrm{~A}$ & $109.90(19)$ & $\mathrm{O} 1 \mathrm{~B}-\mathrm{C} 2 \mathrm{~B}-\mathrm{C} 1 \mathrm{~B}$ & $110.36(18)$ \\
\hline $\mathrm{C} 7 \mathrm{~A}-\mathrm{C} 2 \mathrm{~A}-\mathrm{C} 1 \mathrm{~A}$ & $109.93(19)$ & $\mathrm{C} 7 \mathrm{~B}-\mathrm{C} 2 \mathrm{~B}-\mathrm{C} 1 \mathrm{~B}$ & $110.11(18)$ \\
\hline $\mathrm{C} 3 \mathrm{~A}-\mathrm{C} 2 \mathrm{~A}-\mathrm{C} 1 \mathrm{~A}$ & $112.0(2)$ & $\mathrm{C} 3 \mathrm{~B}-\mathrm{C} 2 \mathrm{~B}-\mathrm{C} 1 \mathrm{~B}$ & $111.5(2)$ \\
\hline $\mathrm{C} 4 \mathrm{~A}-\mathrm{C} 3 \mathrm{~A}-\mathrm{C} 2 \mathrm{~A}$ & $109.8(2)$ & $\mathrm{C} 4 \mathrm{~B}-\mathrm{C} 3 \mathrm{~B}-\mathrm{C} 2 \mathrm{~B}$ & $110.0(2)$ \\
\hline $\mathrm{C} 4 \mathrm{~A}-\mathrm{C} 3 \mathrm{~A}-\mathrm{H} 3 \mathrm{AA}$ & 109.7 & $\mathrm{C} 4 \mathrm{~B}-\mathrm{C} 3 \mathrm{~B}-\mathrm{H} 3 \mathrm{BA}$ & 109.7 \\
\hline $\mathrm{C} 2 \mathrm{~A}-\mathrm{C} 3 \mathrm{~A}-\mathrm{H} 3 \mathrm{AA}$ & 109.7 & $\mathrm{C} 2 \mathrm{~B}-\mathrm{C} 3 \mathrm{~B}-\mathrm{H} 3 \mathrm{BA}$ & 109.7 \\
\hline $\mathrm{C} 4 \mathrm{~A}-\mathrm{C} 3 \mathrm{~A}-\mathrm{H} 3 \mathrm{AB}$ & 109.7 & $\mathrm{C} 4 \mathrm{~B}-\mathrm{C} 3 \mathrm{~B}-\mathrm{H} 3 \mathrm{BB}$ & 109.7 \\
\hline $\mathrm{C} 2 \mathrm{~A}-\mathrm{C} 3 \mathrm{~A}-\mathrm{H} 3 \mathrm{AB}$ & 109.7 & $\mathrm{C} 2 \mathrm{~B}-\mathrm{C} 3 \mathrm{~B}-\mathrm{H} 3 \mathrm{BB}$ & 109.7 \\
\hline $\mathrm{H} 3 \mathrm{AA}-\mathrm{C} 3 \mathrm{~A}-\mathrm{H} 3 \mathrm{AB}$ & 108.2 & $\mathrm{H} 3 \mathrm{BA}-\mathrm{C} 3 \mathrm{~B}-\mathrm{H} 3 \mathrm{BB}$ & 108.2 \\
\hline $\mathrm{N} 1 \mathrm{~A}-\mathrm{C} 4 \mathrm{~A}-\mathrm{C} 3 \mathrm{~A}$ & $113.3(2)$ & $\mathrm{N} 1 \mathrm{~B}-\mathrm{C} 4 \mathrm{~B}-\mathrm{C} 3 \mathrm{~B}$ & $112.3(2)$ \\
\hline $\mathrm{N} 1 \mathrm{~A}-\mathrm{C} 4 \mathrm{~A}-\mathrm{H} 4 \mathrm{AA}$ & 108.9 & $\mathrm{~N} 1 \mathrm{~B}-\mathrm{C} 4 \mathrm{~B}-\mathrm{H} 4 \mathrm{BA}$ & 109.2 \\
\hline $\mathrm{C} 3 \mathrm{~A}-\mathrm{C} 4 \mathrm{~A}-\mathrm{H} 4 \mathrm{AA}$ & 108.9 & $\mathrm{C} 3 \mathrm{~B}-\mathrm{C} 4 \mathrm{~B}-\mathrm{H} 4 \mathrm{BA}$ & 109.2 \\
\hline $\mathrm{N} 1 \mathrm{~A}-\mathrm{C} 4 \mathrm{~A}-\mathrm{H} 4 \mathrm{AB}$ & 108.9 & $\mathrm{~N} 1 \mathrm{~B}-\mathrm{C} 4 \mathrm{~B}-\mathrm{H} 4 \mathrm{BB}$ & 109.2 \\
\hline $\mathrm{C} 3 \mathrm{~A}-\mathrm{C} 4 \mathrm{~A}-\mathrm{H} 4 \mathrm{AB}$ & 108.9 & $\mathrm{C} 3 \mathrm{~B}-\mathrm{C} 4 \mathrm{~B}-\mathrm{H} 4 \mathrm{BB}$ & 109.2 \\
\hline $\mathrm{H} 4 \mathrm{AA}-\mathrm{C} 4 \mathrm{~A}-\mathrm{H} 4 \mathrm{AB}$ & 107.7 & $\mathrm{H} 4 \mathrm{BA}-\mathrm{C} 4 \mathrm{~B}-\mathrm{H} 4 \mathrm{BB}$ & 107.9 \\
\hline $\mathrm{N} 1 \mathrm{~A}-\mathrm{C} 5 \mathrm{~A}-\mathrm{H} 5 \mathrm{AA}$ & 109.5 & $\mathrm{~N} 1 \mathrm{~B}-\mathrm{C} 5 \mathrm{~B}-\mathrm{H} 5 \mathrm{BA}$ & 109.5 \\
\hline $\mathrm{N} 1 \mathrm{~A}-\mathrm{C} 5 \mathrm{~A}-\mathrm{H} 5 \mathrm{AB}$ & 109.5 & $\mathrm{~N} 1 \mathrm{~B}-\mathrm{C} 5 \mathrm{~B}-\mathrm{H} 5 \mathrm{BB}$ & 109.5 \\
\hline $\mathrm{H} 5 \mathrm{AA}-\mathrm{C} 5 \mathrm{~A}-\mathrm{H} 5 \mathrm{AB}$ & 109.5 & $\mathrm{H} 5 \mathrm{BA}-\mathrm{C} 5 \mathrm{~B}-\mathrm{H} 5 \mathrm{BB}$ & 109.5 \\
\hline $\mathrm{N} 1 \mathrm{~A}-\mathrm{C} 5 \mathrm{~A}-\mathrm{H} 5 \mathrm{AC}$ & 109.5 & $\mathrm{~N} 1 \mathrm{~B}-\mathrm{C} 5 \mathrm{~B}-\mathrm{H} 5 \mathrm{BC}$ & 109.5 \\
\hline $\mathrm{H} 5 \mathrm{AA}-\mathrm{C} 5 \mathrm{~A}-\mathrm{H} 5 \mathrm{AC}$ & 109.5 & $\mathrm{H} 5 \mathrm{BA}-\mathrm{C} 5 \mathrm{~B}-\mathrm{H} 5 \mathrm{BC}$ & 109.5 \\
\hline $\mathrm{H} 5 \mathrm{AB}-\mathrm{C} 5 \mathrm{~A}-\mathrm{H} 5 \mathrm{AC}$ & 109.5 & $\mathrm{H} 5 \mathrm{BB}-\mathrm{C} 5 \mathrm{~B}-\mathrm{H} 5 \mathrm{BC}$ & 109.5 \\
\hline N1A-C6A-H6AA & 109.5 & $\mathrm{~N} 1 \mathrm{~B}-\mathrm{C} 6 \mathrm{~B}-\mathrm{H} 6 \mathrm{BA}$ & 109.5 \\
\hline $\mathrm{N} 1 \mathrm{~A}-\mathrm{C} 6 \mathrm{~A}-\mathrm{H} 6 \mathrm{AB}$ & 109.5 & $\mathrm{~N} 1 \mathrm{~B}-\mathrm{C} 6 \mathrm{~B}-\mathrm{H} 6 \mathrm{BB}$ & 109.5 \\
\hline H6AA-C6A-H6AB & 109.5 & $\mathrm{H} 6 \mathrm{BA}-\mathrm{C} 6 \mathrm{~B}-\mathrm{H} 6 \mathrm{BB}$ & 109.5 \\
\hline $\mathrm{N} 1 \mathrm{~A}-\mathrm{C} 6 \mathrm{~A}-\mathrm{H} 6 \mathrm{AC}$ & 109.5 & $\mathrm{~N} 1 \mathrm{~B}-\mathrm{C} 6 \mathrm{~B}-\mathrm{H} 6 \mathrm{BC}$ & 109.5 \\
\hline H6AA-C6A-H6AC & 109.5 & $\mathrm{H} 6 \mathrm{BA}-\mathrm{C} 6 \mathrm{~B}-\mathrm{H} 6 \mathrm{BC}$ & 109.5 \\
\hline $\mathrm{H} 6 \mathrm{AB}-\mathrm{C} 6 \mathrm{~A}-\mathrm{H} 6 \mathrm{AC}$ & 109.5 & $\mathrm{H} 6 \mathrm{BB}-\mathrm{C} 6 \mathrm{~B}-\mathrm{H} 6 \mathrm{BC}$ & 109.5 \\
\hline $\mathrm{C} 8 \mathrm{~A}-\mathrm{C} 7 \mathrm{~A}-\mathrm{C} 16 \mathrm{~A}$ & $118.2(3)$ & $\mathrm{C} 8 \mathrm{~B}-\mathrm{C} 7 \mathrm{~B}-\mathrm{C} 16 \mathrm{~B}$ & $118.6(2)$ \\
\hline $\mathrm{C} 8 \mathrm{~A}-\mathrm{C} 7 \mathrm{~A}-\mathrm{C} 2 \mathrm{~A}$ & $117.8(2)$ & $\mathrm{C} 8 \mathrm{~B}-\mathrm{C} 7 \mathrm{~B}-\mathrm{C} 2 \mathrm{~B}$ & $117.9(2)$ \\
\hline $\mathrm{C} 16 \mathrm{~A}-\mathrm{C} 7 \mathrm{~A}-\mathrm{C} 2 \mathrm{~A}$ & $123.9(2)$ & $\mathrm{C} 16 \mathrm{~B}-\mathrm{C} 7 \mathrm{~B}-\mathrm{C} 2 \mathrm{~B}$ & $123.3(2)$ \\
\hline $\mathrm{C} 7 \mathrm{~A}-\mathrm{C} 8 \mathrm{~A}-\mathrm{C} 9 \mathrm{~A}$ & $122.6(3)$ & $\mathrm{C} 7 \mathrm{~B}-\mathrm{C} 8 \mathrm{~B}-\mathrm{C} 9 \mathrm{~B}$ & $122.7(3)$ \\
\hline $\mathrm{C} 7 \mathrm{~A}-\mathrm{C} 8 \mathrm{~A}-\mathrm{H} 8 \mathrm{~A}$ & 118.7 & $\mathrm{C} 7 \mathrm{~B}-\mathrm{C} 8 \mathrm{~B}-\mathrm{H} 8 \mathrm{~B}$ & 118.6 \\
\hline $\mathrm{C} 9 \mathrm{~A}-\mathrm{C} 8 \mathrm{~A}-\mathrm{H} 8 \mathrm{~A}$ & 118.7 & $\mathrm{C} 9 \mathrm{~B}-\mathrm{C} 8 \mathrm{~B}-\mathrm{H} 8 \mathrm{~B}$ & 118.6 \\
\hline $\mathrm{C} 10 \mathrm{~A}-\mathrm{C} 9 \mathrm{~A}-\mathrm{C} 8 \mathrm{~A}$ & $120.3(3)$ & $\mathrm{C} 10 \mathrm{~B}-\mathrm{C} 9 \mathrm{~B}-\mathrm{C} 8 \mathrm{~B}$ & $119.7(3)$ \\
\hline $\mathrm{C} 10 \mathrm{~A}-\mathrm{C} 9 \mathrm{~A}-\mathrm{H} 9 \mathrm{~A}$ & 119.9 & $\mathrm{C} 10 \mathrm{~B}-\mathrm{C} 9 \mathrm{~B}-\mathrm{H} 9 \mathrm{~B}$ & 120.2 \\
\hline $\mathrm{C} 8 \mathrm{~A}-\mathrm{C} 9 \mathrm{~A}-\mathrm{H} 9 \mathrm{~A}$ & 119.9 & $\mathrm{C} 8 \mathrm{~B}-\mathrm{C} 9 \mathrm{~B}-\mathrm{H} 9 \mathrm{~B}$ & 120.2 \\
\hline
\end{tabular}




\begin{tabular}{|c|c|c|c|}
\hline $\mathrm{C} 9 \mathrm{~A}-\mathrm{C} 10 \mathrm{~A}-\mathrm{C} 11 \mathrm{~A}$ & $120.1(3)$ & $\mathrm{C} 9 \mathrm{~B}-\mathrm{C} 10 \mathrm{~B}-\mathrm{C} 11 \mathrm{~B}$ & $120.6(3)$ \\
\hline $\mathrm{C} 9 \mathrm{~A}-\mathrm{C} 10 \mathrm{~A}-\mathrm{H} 10 \mathrm{~A}$ & 120.0 & $\mathrm{C} 9 \mathrm{~B}-\mathrm{C} 10 \mathrm{~B}-\mathrm{H} 10 \mathrm{~B}$ & 119.7 \\
\hline $\mathrm{C} 11 \mathrm{~A}-\mathrm{C} 10 \mathrm{~A}-\mathrm{H} 10 \mathrm{~A}$ & 120.0 & $\mathrm{C} 11 \mathrm{~B}-\mathrm{C} 10 \mathrm{~B}-\mathrm{H} 10 \mathrm{~B}$ & 119.7 \\
\hline $\mathrm{C} 12 \mathrm{~A}-\mathrm{C} 11 \mathrm{~A}-\mathrm{C} 10 \mathrm{~A}$ & $119.7(3)$ & $\mathrm{C} 10 \mathrm{~B}-\mathrm{C} 11 \mathrm{~B}-\mathrm{C} 12 \mathrm{~B}$ & $119.7(3)$ \\
\hline $\mathrm{C} 12 \mathrm{~A}-\mathrm{C} 11 \mathrm{~A}-\mathrm{C} 16 \mathrm{~A}$ & $120.0(3)$ & $\mathrm{C} 10 \mathrm{~B}-\mathrm{C} 11 \mathrm{~B}-\mathrm{C} 16 \mathrm{~B}$ & $120.3(3)$ \\
\hline $\mathrm{C} 10 \mathrm{~A}-\mathrm{C} 11 \mathrm{~A}-\mathrm{C} 16 \mathrm{~A}$ & $120.3(3)$ & $\mathrm{C} 12 \mathrm{~B}-\mathrm{C} 11 \mathrm{~B}-\mathrm{C} 16 \mathrm{~B}$ & $120.0(4)$ \\
\hline $\mathrm{C} 13 \mathrm{~A}-\mathrm{C} 12 \mathrm{~A}-\mathrm{C} 11 \mathrm{~A}$ & $121.3(3)$ & $\mathrm{C} 13 \mathrm{~B}-\mathrm{C} 12 \mathrm{~B}-\mathrm{C} 11 \mathrm{~B}$ & $121.1(3)$ \\
\hline $\mathrm{C} 13 \mathrm{~A}-\mathrm{C} 12 \mathrm{~A}-\mathrm{H} 12 \mathrm{~A}$ & 119.4 & $\mathrm{C} 13 \mathrm{~B}-\mathrm{C} 12 \mathrm{~B}-\mathrm{H} 12 \mathrm{~B}$ & 119.5 \\
\hline $\mathrm{C} 11 \mathrm{~A}-\mathrm{C} 12 \mathrm{~A}-\mathrm{H} 12 \mathrm{~A}$ & 119.4 & $\mathrm{C} 11 \mathrm{~B}-\mathrm{C} 12 \mathrm{~B}-\mathrm{H} 12 \mathrm{~B}$ & 119.5 \\
\hline $\mathrm{C} 12 \mathrm{~A}-\mathrm{C} 13 \mathrm{~A}-\mathrm{C} 14 \mathrm{~A}$ & $119.8(3)$ & $\mathrm{C} 12 \mathrm{~B}-\mathrm{C} 13 \mathrm{~B}-\mathrm{C} 14 \mathrm{~B}$ & $119.5(3)$ \\
\hline $\mathrm{C} 12 \mathrm{~A}-\mathrm{C} 13 \mathrm{~A}-\mathrm{H} 13 \mathrm{~A}$ & 120.1 & $\mathrm{C} 12 \mathrm{~B}-\mathrm{C} 13 \mathrm{~B}-\mathrm{H} 13 \mathrm{~B}$ & 120.3 \\
\hline $\mathrm{C} 14 \mathrm{~A}-\mathrm{C} 13 \mathrm{~A}-\mathrm{H} 13 \mathrm{~A}$ & 120.1 & $\mathrm{C} 14 \mathrm{~B}-\mathrm{C} 13 \mathrm{~B}-\mathrm{H} 13 \mathrm{~B}$ & 120.3 \\
\hline $\mathrm{C} 15 \mathrm{~A}-\mathrm{C} 14 \mathrm{~A}-\mathrm{C} 13 \mathrm{~A}$ & $120.9(4)$ & $\mathrm{C} 15 \mathrm{~B}-\mathrm{C} 14 \mathrm{~B}-\mathrm{C} 13 \mathrm{~B}$ & $120.9(4)$ \\
\hline $\mathrm{C} 15 \mathrm{~A}-\mathrm{C} 14 \mathrm{~A}-\mathrm{H} 14 \mathrm{~A}$ & 119.6 & $\mathrm{C} 15 \mathrm{~B}-\mathrm{C} 14 \mathrm{~B}-\mathrm{H} 14 \mathrm{~B}$ & 119.6 \\
\hline $\mathrm{C} 13 \mathrm{~A}-\mathrm{C} 14 \mathrm{~A}-\mathrm{H} 14 \mathrm{~A}$ & 119.6 & $\mathrm{C} 13 \mathrm{~B}-\mathrm{C} 14 \mathrm{~B}-\mathrm{H} 14 \mathrm{~B}$ & 119.6 \\
\hline $\mathrm{C} 14 \mathrm{~A}-\mathrm{C} 15 \mathrm{~A}-\mathrm{C} 16 \mathrm{~A}$ & $121.3(3)$ & $\mathrm{C} 14 \mathrm{~B}-\mathrm{C} 15 \mathrm{~B}-\mathrm{C} 16 \mathrm{~B}$ & $121.6(3)$ \\
\hline $\mathrm{C} 14 \mathrm{~A}-\mathrm{C} 15 \mathrm{~A}-\mathrm{H} 15 \mathrm{~A}$ & 119.3 & $\mathrm{C} 14 \mathrm{~B}-\mathrm{C} 15 \mathrm{~B}-\mathrm{H} 15 \mathrm{~B}$ & 119.2 \\
\hline $\mathrm{C} 16 \mathrm{~A}-\mathrm{C} 15 \mathrm{~A}-\mathrm{H} 15 \mathrm{~A}$ & 119.3 & $\mathrm{C} 16 \mathrm{~B}-\mathrm{C} 15 \mathrm{~B}-\mathrm{H} 15 \mathrm{~B}$ & 119.2 \\
\hline $\mathrm{C} 15 \mathrm{~A}-\mathrm{C} 16 \mathrm{~A}-\mathrm{C} 11 \mathrm{~A}$ & $116.6(3)$ & $\mathrm{C} 15 \mathrm{~B}-\mathrm{C} 16 \mathrm{~B}-\mathrm{C} 11 \mathrm{~B}$ & $116.9(3)$ \\
\hline $\mathrm{C} 15 \mathrm{~A}-\mathrm{C} 16 \mathrm{~A}-\mathrm{C} 7 \mathrm{~A}$ & $124.8(3)$ & $\mathrm{C} 15 \mathrm{~B}-\mathrm{C} 16 \mathrm{~B}-\mathrm{C} 7 \mathrm{~B}$ & $125.0(2)$ \\
\hline $\mathrm{C} 11 \mathrm{~A}-\mathrm{C} 16 \mathrm{~A}-\mathrm{C} 7 \mathrm{~A}$ & $118.5(3)$ & $\mathrm{C} 11 \mathrm{~B}-\mathrm{C} 16 \mathrm{~B}-\mathrm{C} 7 \mathrm{~B}$ & $118.1(3)$ \\
\hline $\mathrm{C} 22 \mathrm{~A}-\mathrm{C} 17 \mathrm{~A}-\mathrm{C} 18 \mathrm{~A}$ & $117.2(3)$ & $\mathrm{C} 18 \mathrm{~B}-\mathrm{C} 17 \mathrm{~B}-\mathrm{C} 22 \mathrm{~B}$ & $117.7(2)$ \\
\hline $\mathrm{C} 22 \mathrm{~A}-\mathrm{C} 17 \mathrm{~A}-\mathrm{C} 1 \mathrm{~A}$ & $126.7(2)$ & $\mathrm{C} 18 \mathrm{~B}-\mathrm{C} 17 \mathrm{~B}-\mathrm{C} 1 \mathrm{~B}$ & $126.6(2)$ \\
\hline $\mathrm{C} 18 \mathrm{~A}-\mathrm{C} 17 \mathrm{~A}-\mathrm{C} 1 \mathrm{~A}$ & $116.0(3)$ & $\mathrm{C} 22 \mathrm{~B}-\mathrm{C} 17 \mathrm{~B}-\mathrm{C} 1 \mathrm{~B}$ & $115.6(2)$ \\
\hline $\mathrm{C} 17 \mathrm{~A}-\mathrm{C} 18 \mathrm{~A}-\mathrm{C} 19 \mathrm{~A}$ & $121.2(4)$ & $\mathrm{C} 17 \mathrm{~B}-\mathrm{C} 18 \mathrm{~B}-\mathrm{C} 19 \mathrm{~B}$ & $120.5(3)$ \\
\hline $\mathrm{C} 17 \mathrm{~A}-\mathrm{C} 18 \mathrm{~A}-\mathrm{H} 18 \mathrm{~A}$ & 119.4 & $\mathrm{C} 17 \mathrm{~B}-\mathrm{C} 18 \mathrm{~B}-\mathrm{H} 18 \mathrm{~B}$ & 119.8 \\
\hline $\mathrm{C} 19 \mathrm{~A}-\mathrm{C} 18 \mathrm{~A}-\mathrm{H} 18 \mathrm{~A}$ & 119.4 & $\mathrm{C} 19 \mathrm{~B}-\mathrm{C} 18 \mathrm{~B}-\mathrm{H} 18 \mathrm{~B}$ & 119.8 \\
\hline $\mathrm{C} 20 \mathrm{~A}-\mathrm{C} 19 \mathrm{~A}-\mathrm{C} 18 \mathrm{~A}$ & $120.7(3)$ & $\mathrm{C} 20 \mathrm{~B}-\mathrm{C} 19 \mathrm{~B}-\mathrm{C} 18 \mathrm{~B}$ & $121.1(3)$ \\
\hline $\mathrm{C} 20 \mathrm{~A}-\mathrm{C} 19 \mathrm{~A}-\mathrm{H} 19 \mathrm{~A}$ & 119.6 & $\mathrm{C} 20 \mathrm{~B}-\mathrm{C} 19 \mathrm{~B}-\mathrm{H} 19 \mathrm{~B}$ & 119.4 \\
\hline $\mathrm{C} 18 \mathrm{~A}-\mathrm{C} 19 \mathrm{~A}-\mathrm{H} 19 \mathrm{~A}$ & 119.6 & $\mathrm{C} 18 \mathrm{~B}-\mathrm{C} 19 \mathrm{~B}-\mathrm{H} 19 \mathrm{~B}$ & 119.4 \\
\hline $\mathrm{C} 21 \mathrm{~A}-\mathrm{C} 20 \mathrm{~A}-\mathrm{C} 19 \mathrm{~A}$ & $119.1(3)$ & $\mathrm{C} 19 \mathrm{~B}-\mathrm{C} 20 \mathrm{~B}-\mathrm{C} 21 \mathrm{~B}$ & $118.9(3)$ \\
\hline $\mathrm{C} 21 \mathrm{~A}-\mathrm{C} 20 \mathrm{~A}-\mathrm{H} 20 \mathrm{~A}$ & 120.5 & $\mathrm{C} 19 \mathrm{~B}-\mathrm{C} 20 \mathrm{~B}-\mathrm{H} 20 \mathrm{~B}$ & 120.6 \\
\hline $\mathrm{C} 19 \mathrm{~A}-\mathrm{C} 20 \mathrm{~A}-\mathrm{H} 20 \mathrm{~A}$ & 120.5 & $\mathrm{C} 21 \mathrm{~B}-\mathrm{C} 20 \mathrm{~B}-\mathrm{H} 20 \mathrm{~B}$ & 120.6 \\
\hline $\mathrm{C} 20 \mathrm{~A}-\mathrm{C} 21 \mathrm{~A}-\mathrm{C} 22 \mathrm{~A}$ & $120.7(4)$ & $\mathrm{C} 20 \mathrm{~B}-\mathrm{C} 21 \mathrm{~B}-\mathrm{C} 22 \mathrm{~B}$ & $120.5(3)$ \\
\hline $\mathrm{C} 20 \mathrm{~A}-\mathrm{C} 21 \mathrm{~A}-\mathrm{H} 21 \mathrm{~A}$ & 119.6 & $\mathrm{C} 20 \mathrm{~B}-\mathrm{C} 21 \mathrm{~B}-\mathrm{H} 21 \mathrm{~B}$ & 119.8 \\
\hline $\mathrm{C} 22 \mathrm{~A}-\mathrm{C} 21 \mathrm{~A}-\mathrm{H} 21 \mathrm{~A}$ & 119.6 & $\mathrm{C} 22 \mathrm{~B}-\mathrm{C} 21 \mathrm{~B}-\mathrm{H} 21 \mathrm{~B}$ & 119.8 \\
\hline $\mathrm{C} 17 \mathrm{~A}-\mathrm{C} 22 \mathrm{~A}-\mathrm{C} 21 \mathrm{~A}$ & $121.1(3)$ & $\mathrm{C} 21 \mathrm{~B}-\mathrm{C} 22 \mathrm{~B}-\mathrm{C} 17 \mathrm{~B}$ & $121.3(3)$ \\
\hline $\mathrm{C} 17 \mathrm{~A}-\mathrm{C} 22 \mathrm{~A}-\mathrm{H} 22 \mathrm{~A}$ & 119.5 & $\mathrm{C} 21 \mathrm{~B}-\mathrm{C} 22 \mathrm{~B}-\mathrm{H} 22 \mathrm{~B}$ & 119.3 \\
\hline $\mathrm{C} 21 \mathrm{~A}-\mathrm{C} 22 \mathrm{~A}-\mathrm{H} 22 \mathrm{~A}$ & 119.5 & $\mathrm{C} 17 \mathrm{~B}-\mathrm{C} 22 \mathrm{~B}-\mathrm{H} 22 \mathrm{~B}$ & 119.3 \\
\hline $\mathrm{C} 24 \mathrm{~A}-\mathrm{C} 23 \mathrm{~A}-\mathrm{C} 31 \mathrm{~A}$ & $115.7(3)$ & $\mathrm{C} 24 \mathrm{~B}-\mathrm{C} 23 \mathrm{~B}-\mathrm{C} 31 \mathrm{~B}$ & $115.9(2)$ \\
\hline $\mathrm{C} 24 \mathrm{~A}-\mathrm{C} 23 \mathrm{~A}-\mathrm{C} 1 \mathrm{~A}$ & $123.6(2)$ & $\mathrm{C} 24 \mathrm{~B}-\mathrm{C} 23 \mathrm{~B}-\mathrm{C} 1 \mathrm{~B}$ & $123.5(2)$ \\
\hline $\mathrm{C} 31 \mathrm{~A}-\mathrm{C} 23 \mathrm{~A}-\mathrm{C} 1 \mathrm{~A}$ & $120.7(2)$ & $\mathrm{C} 31 \mathrm{~B}-\mathrm{C} 23 \mathrm{~B}-\mathrm{C} 1 \mathrm{~B}$ & $120.5(2)$ \\
\hline $\mathrm{C} 23 \mathrm{~A}-\mathrm{C} 24 \mathrm{~A}-\mathrm{C} 25 \mathrm{~A}$ & $120.7(2)$ & $\mathrm{C} 23 \mathrm{~B}-\mathrm{C} 24 \mathrm{~B}-\mathrm{C} 25 \mathrm{~B}$ & $120.4(2)$ \\
\hline $\mathrm{C} 23 \mathrm{~A}-\mathrm{C} 24 \mathrm{~A}-\mathrm{H} 24 \mathrm{~A}$ & 119.7 & $\mathrm{C} 23 \mathrm{~B}-\mathrm{C} 24 \mathrm{~B}-\mathrm{H} 24 \mathrm{~B}$ & 119.8 \\
\hline $\mathrm{C} 25 \mathrm{~A}-\mathrm{C} 24 \mathrm{~A}-\mathrm{H} 24 \mathrm{~A}$ & 119.7 & $\mathrm{C} 25 \mathrm{~B}-\mathrm{C} 24 \mathrm{~B}-\mathrm{H} 24 \mathrm{~B}$ & 119.8 \\
\hline $\mathrm{C} 30 \mathrm{~A}-\mathrm{C} 25 \mathrm{~A}-\mathrm{C} 26 \mathrm{~A}$ & $119.5(3)$ & $\mathrm{C} 24 \mathrm{~B}-\mathrm{C} 25 \mathrm{~B}-\mathrm{C} 26 \mathrm{~B}$ & $122.1(2)$ \\
\hline $\mathrm{C} 30 \mathrm{~A}-\mathrm{C} 25 \mathrm{~A}-\mathrm{C} 24 \mathrm{~A}$ & $118.2(3)$ & $\mathrm{C} 24 \mathrm{~B}-\mathrm{C} 25 \mathrm{~B}-\mathrm{C} 30 \mathrm{~B}$ & $118.2(2)$ \\
\hline $\mathrm{C} 26 \mathrm{~A}-\mathrm{C} 25 \mathrm{~A}-\mathrm{C} 24 \mathrm{~A}$ & $122.3(3)$ & $\mathrm{C} 26 \mathrm{~B}-\mathrm{C} 25 \mathrm{~B}-\mathrm{C} 30 \mathrm{~B}$ & $119.7(2)$ \\
\hline
\end{tabular}




\begin{tabular}{|c|c|}
\hline $\mathrm{C} 27 \mathrm{~A}-\mathrm{C} 26 \mathrm{~A}-\mathrm{C} 25 \mathrm{~A}$ & $118.9(3)$ \\
\hline $\mathrm{C} 27 \mathrm{~A}-\mathrm{C} 26 \mathrm{~A}-\mathrm{H} 26 \mathrm{~A}$ & 120.6 \\
\hline $\mathrm{C} 25 \mathrm{~A}-\mathrm{C} 26 \mathrm{~A}-\mathrm{H} 26 \mathrm{~A}$ & 120.6 \\
\hline $\mathrm{C} 26 \mathrm{~A}-\mathrm{C} 27 \mathrm{~A}-\mathrm{C} 28 \mathrm{~A}$ & $122.8(3)$ \\
\hline $\mathrm{C} 26 \mathrm{~A}-\mathrm{C} 27 \mathrm{~A}-\mathrm{Br} 1 \mathrm{~A}$ & $119.0(3)$ \\
\hline $\mathrm{C} 28 \mathrm{~A}-\mathrm{C} 27 \mathrm{~A}-\mathrm{Br} 1 \mathrm{~A}$ & $118.2(3)$ \\
\hline $\mathrm{C} 29 \mathrm{~A}-\mathrm{C} 28 \mathrm{~A}-\mathrm{C} 27 \mathrm{~A}$ & $118.6(3)$ \\
\hline $\mathrm{C} 29 \mathrm{~A}-\mathrm{C} 28 \mathrm{~A}-\mathrm{H} 28 \mathrm{~A}$ & 120.7 \\
\hline $\mathrm{C} 27 \mathrm{~A}-\mathrm{C} 28 \mathrm{~A}-\mathrm{H} 28 \mathrm{~A}$ & 120.7 \\
\hline $\mathrm{C} 28 \mathrm{~A}-\mathrm{C} 29 \mathrm{~A}-\mathrm{C} 30 \mathrm{~A}$ & $121.3(3)$ \\
\hline $\mathrm{C} 28 \mathrm{~A}-\mathrm{C} 29 \mathrm{~A}-\mathrm{H} 29 \mathrm{~A}$ & 119.4 \\
\hline $\mathrm{C} 30 \mathrm{~A}-\mathrm{C} 29 \mathrm{~A}-\mathrm{H} 29 \mathrm{~A}$ & 119.4 \\
\hline $\mathrm{N} 2 \mathrm{~A}-\mathrm{C} 30 \mathrm{~A}-\mathrm{C} 25 \mathrm{~A}$ & $121.7(3)$ \\
\hline $\mathrm{N} 2 \mathrm{~A}-\mathrm{C} 30 \mathrm{~A}-\mathrm{C} 29 \mathrm{~A}$ & $119.4(3)$ \\
\hline $\mathrm{C} 25 \mathrm{~A}-\mathrm{C} 30 \mathrm{~A}-\mathrm{C} 29 \mathrm{~A}$ & $118.9(3)$ \\
\hline $\mathrm{N} 2 \mathrm{~A}-\mathrm{C} 31 \mathrm{~A}-\mathrm{O} 2 \mathrm{~A}$ & $120.2(3)$ \\
\hline $\mathrm{N} 2 \mathrm{~A}-\mathrm{C} 31 \mathrm{~A}-\mathrm{C} 23 \mathrm{~A}$ & $126.1(3)$ \\
\hline $\mathrm{O} 2 \mathrm{~A}-\mathrm{C} 31 \mathrm{~A}-\mathrm{C} 23 \mathrm{~A}$ & $113.7(3)$ \\
\hline $\mathrm{O} 2 \mathrm{~A}-\mathrm{C} 32 \mathrm{~A}-\mathrm{H} 32 \mathrm{~A}$ & 109.5 \\
\hline $\mathrm{O} 2 \mathrm{~A}-\mathrm{C} 32 \mathrm{~A}-\mathrm{H} 32 \mathrm{~B}$ & 109.5 \\
\hline $\mathrm{H} 32 \mathrm{~A}-\mathrm{C} 32 \mathrm{~A}-\mathrm{H} 32 \mathrm{~B}$ & 109.5 \\
\hline $\mathrm{O} 2 \mathrm{~A}-\mathrm{C} 32 \mathrm{~A}-\mathrm{H} 32 \mathrm{C}$ & 109.5 \\
\hline $\mathrm{H} 32 \mathrm{~A}-\mathrm{C} 32 \mathrm{~A}-\mathrm{H} 32 \mathrm{C}$ & 109.5 \\
\hline $\mathrm{H} 32 \mathrm{~B}-\mathrm{C} 32 \mathrm{~A}-\mathrm{H} 32 \mathrm{C}$ & 109.5 \\
\hline $\mathrm{O} 3 \mathrm{~A}-\mathrm{C} 33 \mathrm{~A}-\mathrm{O} 4 \mathrm{~A}$ & $124.3(2)$ \\
\hline $\mathrm{O} 3 \mathrm{~A}-\mathrm{C} 33 \mathrm{~A}-\mathrm{C} 34 \mathrm{~A}$ & $118.4(2)$ \\
\hline $\mathrm{O} 4 \mathrm{~A}-\mathrm{C} 33 \mathrm{~A}-\mathrm{C} 34 \mathrm{~A}$ & $117.4(2)$ \\
\hline $\mathrm{C} 35 \mathrm{~A}-\mathrm{C} 34 \mathrm{~A}-\mathrm{C} 33 \mathrm{~A}$ & $123.4(2)$ \\
\hline $\mathrm{C} 35 \mathrm{~A}-\mathrm{C} 34 \mathrm{~A}-\mathrm{H} 34 \mathrm{~A}$ & 118.3 \\
\hline $\mathrm{C} 33 \mathrm{~A}-\mathrm{C} 34 \mathrm{~A}-\mathrm{H} 34 \mathrm{~A}$ & 118.3 \\
\hline $\mathrm{C} 34 \mathrm{~A}-\mathrm{C} 35 \mathrm{~A}-\mathrm{C} 36 \mathrm{~A}$ & $120.6(2)$ \\
\hline $\mathrm{C} 34 \mathrm{~A}-\mathrm{C} 35 \mathrm{~A}-\mathrm{H} 35 \mathrm{~A}$ & 119.7 \\
\hline $\mathrm{C} 36 \mathrm{~A}-\mathrm{C} 35 \mathrm{~A}-\mathrm{H} 35 \mathrm{~A}$ & 119.7 \\
\hline $\mathrm{O} 6 \mathrm{~A}-\mathrm{C} 36 \mathrm{~A}-\mathrm{O} 5 \mathrm{~A}$ & $124.6(2)$ \\
\hline $\mathrm{O} 6 \mathrm{~A}-\mathrm{C} 36 \mathrm{~A}-\mathrm{C} 35 \mathrm{~A}$ & $123.3(2)$ \\
\hline $\mathrm{O} 5 \mathrm{~A}-\mathrm{C} 36 \mathrm{~A}-\mathrm{C} 35 \mathrm{~A}$ & $112.1(2)$ \\
\hline $\mathrm{C} 23 \mathrm{~A}-\mathrm{C} 1 \mathrm{~A}-\mathrm{C} 2 \mathrm{~A}-\mathrm{O} 1 \mathrm{~A}$ & $-76.0(3)$ \\
\hline $\mathrm{C} 17 \mathrm{~A}-\mathrm{C} 1 \mathrm{~A}-\mathrm{C} 2 \mathrm{~A}-\mathrm{O} 1 \mathrm{~A}$ & $51.5(3)$ \\
\hline $\mathrm{C} 23 \mathrm{~A}-\mathrm{C} 1 \mathrm{~A}-\mathrm{C} 2 \mathrm{~A}-\mathrm{C} 7 \mathrm{~A}$ & $165.5(2)$ \\
\hline $\mathrm{C} 17 \mathrm{~A}-\mathrm{C} 1 \mathrm{~A}-\mathrm{C} 2 \mathrm{~A}-\mathrm{C} 7 \mathrm{~A}$ & $-67.0(3)$ \\
\hline $\mathrm{C} 23 \mathrm{~A}-\mathrm{C} 1 \mathrm{~A}-\mathrm{C} 2 \mathrm{~A}-\mathrm{C} 3 \mathrm{~A}$ & $42.2(3)$ \\
\hline $\mathrm{C} 17 \mathrm{~A}-\mathrm{C} 1 \mathrm{~A}-\mathrm{C} 2 \mathrm{~A}-\mathrm{C} 3 \mathrm{~A}$ & $169.7(2)$ \\
\hline $\mathrm{O} 1 \mathrm{~A}-\mathrm{C} 2 \mathrm{~A}-\mathrm{C} 3 \mathrm{~A}-\mathrm{C} 4 \mathrm{~A}$ & $-63.8(3)$ \\
\hline $\mathrm{C} 7 \mathrm{~A}-\mathrm{C} 2 \mathrm{~A}-\mathrm{C} 3 \mathrm{~A}-\mathrm{C} 4 \mathrm{~A}$ & $53.1(3)$ \\
\hline $\mathrm{C} 1 \mathrm{~A}-\mathrm{C} 2 \mathrm{~A}-\mathrm{C} 3 \mathrm{~A}-\mathrm{C} 4 \mathrm{~A}$ & $176.0(2)$ \\
\hline $\mathrm{C} 5 \mathrm{~A}-\mathrm{N} 1 \mathrm{~A}-\mathrm{C} 4 \mathrm{~A}-\mathrm{C} 3 \mathrm{~A}$ & $82.3(3)$ \\
\hline $\mathrm{C} 6 \mathrm{~A}-\mathrm{N} 1 \mathrm{~A}-\mathrm{C} 4 \mathrm{~A}-\mathrm{C} 3 \mathrm{~A}$ & $-153.2(2)$ \\
\hline
\end{tabular}

\begin{tabular}{|c|c|}
\hline $\mathrm{C} 27 \mathrm{~B}-\mathrm{C} 26 \mathrm{~B}-\mathrm{C} 25 \mathrm{~B}$ & $118.6(2)$ \\
\hline $\mathrm{C} 27 \mathrm{~B}-\mathrm{C} 26 \mathrm{~B}-\mathrm{H} 26 \mathrm{~B}$ & 120.7 \\
\hline $\mathrm{C} 25 \mathrm{~B}-\mathrm{C} 26 \mathrm{~B}-\mathrm{H} 26 \mathrm{~B}$ & 120.7 \\
\hline $\mathrm{C} 26 \mathrm{~B}-\mathrm{C} 27 \mathrm{~B}-\mathrm{C} 28 \mathrm{~B}$ & $123.1(2)$ \\
\hline $\mathrm{C} 26 \mathrm{~B}-\mathrm{C} 27 \mathrm{~B}-\mathrm{Br} 1 \mathrm{~B}$ & $118.9(2)$ \\
\hline $\mathrm{C} 28 \mathrm{~B}-\mathrm{C} 27 \mathrm{~B}-\mathrm{Br} 1 \mathrm{~B}$ & $118.0(2)$ \\
\hline $\mathrm{C} 29 \mathrm{~B}-\mathrm{C} 28 \mathrm{~B}-\mathrm{C} 27 \mathrm{~B}$ & $118.6(2)$ \\
\hline $\mathrm{C} 29 \mathrm{~B}-\mathrm{C} 28 \mathrm{~B}-\mathrm{H} 28 \mathrm{~B}$ & 120.7 \\
\hline $\mathrm{C} 27 \mathrm{~B}-\mathrm{C} 28 \mathrm{~B}-\mathrm{H} 28 \mathrm{~B}$ & 120.7 \\
\hline $\mathrm{C} 28 \mathrm{~B}-\mathrm{C} 29 \mathrm{~B}-\mathrm{C} 30 \mathrm{~B}$ & $120.6(3)$ \\
\hline $\mathrm{C} 28 \mathrm{~B}-\mathrm{C} 29 \mathrm{~B}-\mathrm{H} 29 \mathrm{~B}$ & 119.7 \\
\hline $\mathrm{C} 30 \mathrm{~B}-\mathrm{C} 29 \mathrm{~B}-\mathrm{H} 29 \mathrm{~B}$ & 119.7 \\
\hline $\mathrm{N} 2 \mathrm{~B}-\mathrm{C} 30 \mathrm{~B}-\mathrm{C} 29 \mathrm{~B}$ & $119.0(2)$ \\
\hline $\mathrm{N} 2 \mathrm{~B}-\mathrm{C} 30 \mathrm{~B}-\mathrm{C} 25 \mathrm{~B}$ & $121.8(2)$ \\
\hline $\mathrm{C} 29 \mathrm{~B}-\mathrm{C} 30 \mathrm{~B}-\mathrm{C} 25 \mathrm{~B}$ & $119.3(2)$ \\
\hline $\mathrm{N} 2 \mathrm{~B}-\mathrm{C} 31 \mathrm{~B}-\mathrm{O} 2 \mathrm{~B}$ & $119.4(2)$ \\
\hline $\mathrm{N} 2 \mathrm{~B}-\mathrm{C} 31 \mathrm{~B}-\mathrm{C} 23 \mathrm{~B}$ & $126.0(2)$ \\
\hline $\mathrm{O} 2 \mathrm{~B}-\mathrm{C} 31 \mathrm{~B}-\mathrm{C} 23 \mathrm{~B}$ & $114.7(2)$ \\
\hline $\mathrm{O} 2 \mathrm{~B}-\mathrm{C} 32 \mathrm{~B}-\mathrm{H} 32 \mathrm{D}$ & 109.5 \\
\hline $\mathrm{O} 2 \mathrm{~B}-\mathrm{C} 32 \mathrm{~B}-\mathrm{H} 32 \mathrm{E}$ & 109.5 \\
\hline $\mathrm{H} 32 \mathrm{D}-\mathrm{C} 32 \mathrm{~B}-\mathrm{H} 32 \mathrm{E}$ & 109.5 \\
\hline $\mathrm{O} 2 \mathrm{~B}-\mathrm{C} 32 \mathrm{~B}-\mathrm{H} 32 \mathrm{~F}$ & 109.5 \\
\hline $\mathrm{H} 32 \mathrm{D}-\mathrm{C} 32 \mathrm{~B}-\mathrm{H} 32 \mathrm{~F}$ & 109.5 \\
\hline $\mathrm{H} 32 \mathrm{E}-\mathrm{C} 32 \mathrm{~B}-\mathrm{H} 32 \mathrm{~F}$ & 109.5 \\
\hline $\mathrm{O} 4 \mathrm{~B}-\mathrm{C} 33 \mathrm{~B}-\mathrm{O} 3 \mathrm{~B}$ & $125.1(2)$ \\
\hline $\mathrm{O} 4 \mathrm{~B}-\mathrm{C} 33 \mathrm{~B}-\mathrm{C} 34 \mathrm{~B}$ & $117.6(2)$ \\
\hline $\mathrm{O} 3 \mathrm{~B}-\mathrm{C} 33 \mathrm{~B}-\mathrm{C} 34 \mathrm{~B}$ & $117.3(2)$ \\
\hline $\mathrm{C} 35 \mathrm{~B}-\mathrm{C} 34 \mathrm{~B}-\mathrm{C} 33 \mathrm{~B}$ & $121.8(2)$ \\
\hline $\mathrm{C} 35 \mathrm{~B}-\mathrm{C} 34 \mathrm{~B}-\mathrm{H} 34 \mathrm{~B}$ & 119.1 \\
\hline $\mathrm{C} 33 \mathrm{~B}-\mathrm{C} 34 \mathrm{~B}-\mathrm{H} 34 \mathrm{~B}$ & 119.1 \\
\hline $\mathrm{C} 34 \mathrm{~B}-\mathrm{C} 35 \mathrm{~B}-\mathrm{C} 36 \mathrm{~B}$ & $124.8(2)$ \\
\hline $\mathrm{C} 34 \mathrm{~B}-\mathrm{C} 35 \mathrm{~B}-\mathrm{H} 35 \mathrm{~B}$ & 117.6 \\
\hline $\mathrm{C} 36 \mathrm{~B}-\mathrm{C} 35 \mathrm{~B}-\mathrm{H} 35 \mathrm{~B}$ & 117.6 \\
\hline $\mathrm{O} 5 \mathrm{~B}-\mathrm{C} 36 \mathrm{~B}-\mathrm{O} 6 \mathrm{~B}$ & $123.8(3)$ \\
\hline $\mathrm{O} 5 \mathrm{~B}-\mathrm{C} 36 \mathrm{~B}-\mathrm{C} 35 \mathrm{~B}$ & $121.5(2)$ \\
\hline $\mathrm{O} 6 \mathrm{~B}-\mathrm{C} 36 \mathrm{~B}-\mathrm{C} 35 \mathrm{~B}$ & $114.6(2)$ \\
\hline $\mathrm{C} 23 \mathrm{~B}-\mathrm{C} 1 \mathrm{~B}-\mathrm{C} 2 \mathrm{~B}-\mathrm{O} 1 \mathrm{~B}$ & $-73.1(2)$ \\
\hline $\mathrm{C} 17 \mathrm{~B}-\mathrm{C} 1 \mathrm{~B}-\mathrm{C} 2 \mathrm{~B}-\mathrm{O} 1 \mathrm{~B}$ & $53.6(3)$ \\
\hline $\mathrm{C} 23 \mathrm{~B}-\mathrm{C} 1 \mathrm{~B}-\mathrm{C} 2 \mathrm{~B}-\mathrm{C} 7 \mathrm{~B}$ & $168.5(2)$ \\
\hline $\mathrm{C} 17 \mathrm{~B}-\mathrm{C} 1 \mathrm{~B}-\mathrm{C} 2 \mathrm{~B}-\mathrm{C} 7 \mathrm{~B}$ & $-64.8(3)$ \\
\hline $\mathrm{C} 23 \mathrm{~B}-\mathrm{C} 1 \mathrm{~B}-\mathrm{C} 2 \mathrm{~B}-\mathrm{C} 3 \mathrm{~B}$ & $44.7(3)$ \\
\hline $\mathrm{C} 17 \mathrm{~B}-\mathrm{C} 1 \mathrm{~B}-\mathrm{C} 2 \mathrm{~B}-\mathrm{C} 3 \mathrm{~B}$ & $171.38(19)$ \\
\hline $\mathrm{O} 1 \mathrm{~B}-\mathrm{C} 2 \mathrm{~B}-\mathrm{C} 3 \mathrm{~B}-\mathrm{C} 4 \mathrm{~B}$ & $-64.8(2)$ \\
\hline $\mathrm{C} 7 \mathrm{~B}-\mathrm{C} 2 \mathrm{~B}-\mathrm{C} 3 \mathrm{~B}-\mathrm{C} 4 \mathrm{~B}$ & $51.7(3)$ \\
\hline $\mathrm{C} 1 \mathrm{~B}-\mathrm{C} 2 \mathrm{~B}-\mathrm{C} 3 \mathrm{~B}-\mathrm{C} 4 \mathrm{~B}$ & $174.92(19)$ \\
\hline $\mathrm{C} 6 \mathrm{~B}-\mathrm{N} 1 \mathrm{~B}-\mathrm{C} 4 \mathrm{~B}-\mathrm{C} 3 \mathrm{~B}$ & $-168.1(2)$ \\
\hline $\mathrm{C} 5 \mathrm{~B}-\mathrm{N} 1 \mathrm{~B}-\mathrm{C} 4 \mathrm{~B}-\mathrm{C} 3 \mathrm{~B}$ & $67.9(3)$ \\
\hline
\end{tabular}




\begin{tabular}{|c|c|}
\hline $\mathrm{C} 2 \mathrm{~A}-\mathrm{C} 3 \mathrm{~A}-\mathrm{C} 4 \mathrm{~A}-\mathrm{N} 1 \mathrm{~A}$ & $137.2(2)$ \\
\hline $\mathrm{O} 1 \mathrm{~A}-\mathrm{C} 2 \mathrm{~A}-\mathrm{C} 7 \mathrm{~A}-\mathrm{C} 8 \mathrm{~A}$ & $-0.2(3)$ \\
\hline $\mathrm{C} 3 \mathrm{~A}-\mathrm{C} 2 \mathrm{~A}-\mathrm{C} 7 \mathrm{~A}-\mathrm{C} 8 \mathrm{~A}$ & $-116.3(3)$ \\
\hline $\mathrm{C} 1 \mathrm{~A}-\mathrm{C} 2 \mathrm{~A}-\mathrm{C} 7 \mathrm{~A}-\mathrm{C} 8 \mathrm{~A}$ & $119.5(2)$ \\
\hline $\mathrm{O} 1 \mathrm{~A}-\mathrm{C} 2 \mathrm{~A}-\mathrm{C} 7 \mathrm{~A}-\mathrm{C} 16 \mathrm{~A}$ & $177.1(2)$ \\
\hline $\mathrm{C} 3 \mathrm{~A}-\mathrm{C} 2 \mathrm{~A}-\mathrm{C} 7 \mathrm{~A}-\mathrm{C} 16 \mathrm{~A}$ & $61.0(3)$ \\
\hline $\mathrm{C} 1 \mathrm{~A}-\mathrm{C} 2 \mathrm{~A}-\mathrm{C} 7 \mathrm{~A}-\mathrm{C} 16 \mathrm{~A}$ & $-63.1(3)$ \\
\hline $\mathrm{C} 16 \mathrm{~A}-\mathrm{C} 7 \mathrm{~A}-\mathrm{C} 8 \mathrm{~A}-\mathrm{C} 9 \mathrm{~A}$ & $0.8(4)$ \\
\hline $\mathrm{C} 2 \mathrm{~A}-\mathrm{C} 7 \mathrm{~A}-\mathrm{C} 8 \mathrm{~A}-\mathrm{C} 9 \mathrm{~A}$ & $178.3(3)$ \\
\hline $\mathrm{C} 7 \mathrm{~A}-\mathrm{C} 8 \mathrm{~A}-\mathrm{C} 9 \mathrm{~A}-\mathrm{C} 10 \mathrm{~A}$ & $0.2(5)$ \\
\hline $\mathrm{C} 8 \mathrm{~A}-\mathrm{C} 9 \mathrm{~A}-\mathrm{C} 10 \mathrm{~A}-\mathrm{C} 11 \mathrm{~A}$ & $-0.8(5)$ \\
\hline $\mathrm{C} 9 \mathrm{~A}-\mathrm{C} 10 \mathrm{~A}-\mathrm{C} 11 \mathrm{~A}-\mathrm{C} 12 \mathrm{~A}$ & $-178.6(3)$ \\
\hline $\mathrm{C} 9 \mathrm{~A}-\mathrm{C} 10 \mathrm{~A}-\mathrm{C} 11 \mathrm{~A}-\mathrm{C} 16 \mathrm{~A}$ & $0.5(5)$ \\
\hline $\mathrm{C} 10 \mathrm{~A}-\mathrm{C} 11 \mathrm{~A}-\mathrm{C} 12 \mathrm{~A}-\mathrm{C} 13 \mathrm{~A}$ & $179.2(4)$ \\
\hline $\mathrm{C} 16 \mathrm{~A}-\mathrm{C} 11 \mathrm{~A}-\mathrm{C} 12 \mathrm{~A}-\mathrm{C} 13 \mathrm{~A}$ & $0.2(5)$ \\
\hline $\mathrm{C} 11 \mathrm{~A}-\mathrm{C} 12 \mathrm{~A}-\mathrm{C} 13 \mathrm{~A}-\mathrm{C} 14 \mathrm{~A}$ & $0.3(6)$ \\
\hline $\mathrm{C} 12 \mathrm{~A}-\mathrm{C} 13 \mathrm{~A}-\mathrm{C} 14 \mathrm{~A}-\mathrm{C} 15 \mathrm{~A}$ & $-0.7(6)$ \\
\hline $\mathrm{C} 13 \mathrm{~A}-\mathrm{C} 14 \mathrm{~A}-\mathrm{C} 15 \mathrm{~A}-\mathrm{C} 16 \mathrm{~A}$ & $0.7(5)$ \\
\hline $\mathrm{C} 14 \mathrm{~A}-\mathrm{C} 15 \mathrm{~A}-\mathrm{C} 16 \mathrm{~A}-\mathrm{C} 11 \mathrm{~A}$ & $-0.2(4)$ \\
\hline $\mathrm{C} 14 \mathrm{~A}-\mathrm{C} 15 \mathrm{~A}-\mathrm{C} 16 \mathrm{~A}-\mathrm{C} 7 \mathrm{~A}$ & $-179.9(3)$ \\
\hline $\mathrm{C} 12 \mathrm{~A}-\mathrm{C} 11 \mathrm{~A}-\mathrm{C} 16 \mathrm{~A}-\mathrm{C} 15 \mathrm{~A}$ & $-0.2(4)$ \\
\hline $\mathrm{C} 10 \mathrm{~A}-\mathrm{C} 11 \mathrm{~A}-\mathrm{C} 16 \mathrm{~A}-\mathrm{C} 15 \mathrm{~A}$ & $-179.3(3)$ \\
\hline $\mathrm{C} 12 \mathrm{~A}-\mathrm{C} 11 \mathrm{~A}-\mathrm{C} 16 \mathrm{~A}-\mathrm{C} 7 \mathrm{~A}$ & $179.5(3)$ \\
\hline $\mathrm{C} 10 \mathrm{~A}-\mathrm{C} 11 \mathrm{~A}-\mathrm{C} 16 \mathrm{~A}-\mathrm{C} 7 \mathrm{~A}$ & $0.4(4)$ \\
\hline $\mathrm{C} 8 \mathrm{~A}-\mathrm{C} 7 \mathrm{~A}-\mathrm{C} 16 \mathrm{~A}-\mathrm{C} 15 \mathrm{~A}$ & $178.6(3)$ \\
\hline $\mathrm{C} 2 \mathrm{~A}-\mathrm{C} 7 \mathrm{~A}-\mathrm{C} 16 \mathrm{~A}-\mathrm{C} 15 \mathrm{~A}$ & $1.3(4)$ \\
\hline $\mathrm{C} 8 \mathrm{~A}-\mathrm{C} 7 \mathrm{~A}-\mathrm{C} 16 \mathrm{~A}-\mathrm{C} 11 \mathrm{~A}$ & $-1.1(4)$ \\
\hline $\mathrm{C} 2 \mathrm{~A}-\mathrm{C} 7 \mathrm{~A}-\mathrm{C} 16 \mathrm{~A}-\mathrm{C} 11 \mathrm{~A}$ & $-178.4(2)$ \\
\hline $\mathrm{C} 23 \mathrm{~A}-\mathrm{C} 1 \mathrm{~A}-\mathrm{C} 17 \mathrm{~A}-\mathrm{C} 22 \mathrm{~A}$ & $108.7(3)$ \\
\hline $\mathrm{C} 2 \mathrm{~A}-\mathrm{C} 1 \mathrm{~A}-\mathrm{C} 17 \mathrm{~A}-\mathrm{C} 22 \mathrm{~A}$ & $-19.8(4)$ \\
\hline $\mathrm{C} 23 \mathrm{~A}-\mathrm{C} 1 \mathrm{~A}-\mathrm{C} 17 \mathrm{~A}-\mathrm{C} 18 \mathrm{~A}$ & $-68.6(3)$ \\
\hline $\mathrm{C} 2 \mathrm{~A}-\mathrm{C} 1 \mathrm{~A}-\mathrm{C} 17 \mathrm{~A}-\mathrm{C} 18 \mathrm{~A}$ & $162.8(3)$ \\
\hline $\mathrm{C} 22 \mathrm{~A}-\mathrm{C} 17 \mathrm{~A}-\mathrm{C} 18 \mathrm{~A}-\mathrm{C} 19 \mathrm{~A}$ & $-1.8(5)$ \\
\hline $\mathrm{C} 1 \mathrm{~A}-\mathrm{C} 17 \mathrm{~A}-\mathrm{C} 18 \mathrm{~A}-\mathrm{C} 19 \mathrm{~A}$ & $175.8(3)$ \\
\hline $\mathrm{C} 17 \mathrm{~A}-\mathrm{C} 18 \mathrm{~A}-\mathrm{C} 19 \mathrm{~A}-\mathrm{C} 20 \mathrm{~A}$ & $2.3(6)$ \\
\hline $\mathrm{C} 18 \mathrm{~A}-\mathrm{C} 19 \mathrm{~A}-\mathrm{C} 20 \mathrm{~A}-\mathrm{C} 21 \mathrm{~A}$ & $-0.7(6)$ \\
\hline $\mathrm{C} 19 \mathrm{~A}-\mathrm{C} 20 \mathrm{~A}-\mathrm{C} 21 \mathrm{~A}-\mathrm{C} 22 \mathrm{~A}$ & $-1.3(6)$ \\
\hline $\mathrm{C} 18 \mathrm{~A}-\mathrm{C} 17 \mathrm{~A}-\mathrm{C} 22 \mathrm{~A}-\mathrm{C} 21 \mathrm{~A}$ & $-0.2(5)$ \\
\hline $\mathrm{C} 1 \mathrm{~A}-\mathrm{C} 17 \mathrm{~A}-\mathrm{C} 22 \mathrm{~A}-\mathrm{C} 21 \mathrm{~A}$ & $-177.6(3)$ \\
\hline $\mathrm{C} 20 \mathrm{~A}-\mathrm{C} 21 \mathrm{~A}-\mathrm{C} 22 \mathrm{~A}-\mathrm{C} 17 \mathrm{~A}$ & $1.8(6)$ \\
\hline $\mathrm{C} 17 \mathrm{~A}-\mathrm{C} 1 \mathrm{~A}-\mathrm{C} 23 \mathrm{~A}-\mathrm{C} 24 \mathrm{~A}$ & $-57.4(3)$ \\
\hline $\mathrm{C} 2 \mathrm{~A}-\mathrm{C} 1 \mathrm{~A}-\mathrm{C} 23 \mathrm{~A}-\mathrm{C} 24 \mathrm{~A}$ & $73.8(3)$ \\
\hline $\mathrm{C} 17 \mathrm{~A}-\mathrm{C} 1 \mathrm{~A}-\mathrm{C} 23 \mathrm{~A}-\mathrm{C} 31 \mathrm{~A}$ & $122.5(2)$ \\
\hline $\mathrm{C} 2 \mathrm{~A}-\mathrm{C} 1 \mathrm{~A}-\mathrm{C} 23 \mathrm{~A}-\mathrm{C} 31 \mathrm{~A}$ & $-106.3(3)$ \\
\hline $\mathrm{C} 31 \mathrm{~A}-\mathrm{C} 23 \mathrm{~A}-\mathrm{C} 24 \mathrm{~A}-\mathrm{C} 25 \mathrm{~A}$ & $2.7(3)$ \\
\hline $\mathrm{C} 1 \mathrm{~A}-\mathrm{C} 23 \mathrm{~A}-\mathrm{C} 24 \mathrm{~A}-\mathrm{C} 25 \mathrm{~A}$ & $-177.3(2)$ \\
\hline $\mathrm{C} 23 \mathrm{~A}-\mathrm{C} 24 \mathrm{~A}-\mathrm{C} 25 \mathrm{~A}-\mathrm{C} 30 \mathrm{~A}$ & $-0.3(3)$ \\
\hline $\mathrm{C} 23 \mathrm{~A}-\mathrm{C} 24 \mathrm{~A}-\mathrm{C} 25 \mathrm{~A}-\mathrm{C} 26 \mathrm{~A}$ & $-179.3(2)$ \\
\hline
\end{tabular}

\begin{tabular}{|c|c|}
\hline $\mathrm{C} 2 \mathrm{~B}-\mathrm{C} 3 \mathrm{~B}-\mathrm{C} 4 \mathrm{~B}-\mathrm{N} 1 \mathrm{~B}$ & $133.7(2)$ \\
\hline $\mathrm{O} 1 \mathrm{~B}-\mathrm{C} 2 \mathrm{~B}-\mathrm{C} 7 \mathrm{~B}-\mathrm{C} 8 \mathrm{~B}$ & $-2.6(3)$ \\
\hline $\mathrm{C} 3 \mathrm{~B}-\mathrm{C} 2 \mathrm{~B}-\mathrm{C} 7 \mathrm{~B}-\mathrm{C} 8 \mathrm{~B}$ & $-118.3(2)$ \\
\hline $\mathrm{C} 1 \mathrm{~B}-\mathrm{C} 2 \mathrm{~B}-\mathrm{C} 7 \mathrm{~B}-\mathrm{C} 8 \mathrm{~B}$ & $117.6(2)$ \\
\hline $\mathrm{O} 1 \mathrm{~B}-\mathrm{C} 2 \mathrm{~B}-\mathrm{C} 7 \mathrm{~B}-\mathrm{C} 16 \mathrm{~B}$ & $173.7(2)$ \\
\hline $\mathrm{C} 3 \mathrm{~B}-\mathrm{C} 2 \mathrm{~B}-\mathrm{C} 7 \mathrm{~B}-\mathrm{C} 16 \mathrm{~B}$ & $58.0(3)$ \\
\hline $\mathrm{C} 1 \mathrm{~B}-\mathrm{C} 2 \mathrm{~B}-\mathrm{C} 7 \mathrm{~B}-\mathrm{C} 16 \mathrm{~B}$ & $-66.1(3)$ \\
\hline $\mathrm{C} 16 \mathrm{~B}-\mathrm{C} 7 \mathrm{~B}-\mathrm{C} 8 \mathrm{~B}-\mathrm{C} 9 \mathrm{~B}$ & $0.6(4)$ \\
\hline $\mathrm{C} 2 \mathrm{~B}-\mathrm{C} 7 \mathrm{~B}-\mathrm{C} 8 \mathrm{~B}-\mathrm{C} 9 \mathrm{~B}$ & $177.1(2)$ \\
\hline $\mathrm{C} 7 \mathrm{~B}-\mathrm{C} 8 \mathrm{~B}-\mathrm{C} 9 \mathrm{~B}-\mathrm{C} 10 \mathrm{~B}$ & $-0.1(4)$ \\
\hline $\mathrm{C} 8 \mathrm{~B}-\mathrm{C} 9 \mathrm{~B}-\mathrm{C} 10 \mathrm{~B}-\mathrm{C} 11 \mathrm{~B}$ & $-1.0(5)$ \\
\hline $\mathrm{C} 9 \mathrm{~B}-\mathrm{C} 10 \mathrm{~B}-\mathrm{C} 11 \mathrm{~B}-\mathrm{C} 12 \mathrm{~B}$ & $-177.2(3)$ \\
\hline $\mathrm{C} 9 \mathrm{~B}-\mathrm{C} 10 \mathrm{~B}-\mathrm{C} 11 \mathrm{~B}-\mathrm{C} 16 \mathrm{~B}$ & $1.5(5)$ \\
\hline $\mathrm{C} 10 \mathrm{~B}-\mathrm{C} 11 \mathrm{~B}-\mathrm{C} 12 \mathrm{~B}-\mathrm{C} 13 \mathrm{~B}$ & $178.2(4)$ \\
\hline $\mathrm{C} 16 \mathrm{~B}-\mathrm{C} 11 \mathrm{~B}-\mathrm{C} 12 \mathrm{~B}-\mathrm{C} 13 \mathrm{~B}$ & $-0.5(6)$ \\
\hline $\mathrm{C} 11 \mathrm{~B}-\mathrm{C} 12 \mathrm{~B}-\mathrm{C} 13 \mathrm{~B}-\mathrm{C} 14 \mathrm{~B}$ & $1.3(7)$ \\
\hline $\mathrm{C} 12 \mathrm{~B}-\mathrm{C} 13 \mathrm{~B}-\mathrm{C} 14 \mathrm{~B}-\mathrm{C} 15 \mathrm{~B}$ & $-0.6(7)$ \\
\hline $\mathrm{C} 13 \mathrm{~B}-\mathrm{C} 14 \mathrm{~B}-\mathrm{C} 15 \mathrm{~B}-\mathrm{C} 16 \mathrm{~B}$ & $-1.0(6)$ \\
\hline $\mathrm{C} 14 \mathrm{~B}-\mathrm{C} 15 \mathrm{~B}-\mathrm{C} 16 \mathrm{~B}-\mathrm{C} 11 \mathrm{~B}$ & $1.8(5)$ \\
\hline $\mathrm{C} 14 \mathrm{~B}-\mathrm{C} 15 \mathrm{~B}-\mathrm{C} 16 \mathrm{~B}-\mathrm{C} 7 \mathrm{~B}$ & $-176.9(3)$ \\
\hline $\mathrm{C} 10 \mathrm{~B}-\mathrm{C} 11 \mathrm{~B}-\mathrm{C} 16 \mathrm{~B}-\mathrm{C} 15 \mathrm{~B}$ & $-179.8(3)$ \\
\hline $\mathrm{C} 12 \mathrm{~B}-\mathrm{C} 11 \mathrm{~B}-\mathrm{C} 16 \mathrm{~B}-\mathrm{C} 15 \mathrm{~B}$ & $-1.1(4)$ \\
\hline $\mathrm{C} 10 \mathrm{~B}-\mathrm{C} 11 \mathrm{~B}-\mathrm{C} 16 \mathrm{~B}-\mathrm{C} 7 \mathrm{~B}$ & $-0.9(4)$ \\
\hline $\mathrm{C} 12 \mathrm{~B}-\mathrm{C} 11 \mathrm{~B}-\mathrm{C} 16 \mathrm{~B}-\mathrm{C} 7 \mathrm{~B}$ & $177.8(3)$ \\
\hline $\mathrm{C} 8 \mathrm{~B}-\mathrm{C} 7 \mathrm{~B}-\mathrm{C} 16 \mathrm{~B}-\mathrm{C} 15 \mathrm{~B}$ & $178.6(3)$ \\
\hline $\mathrm{C} 2 \mathrm{~B}-\mathrm{C} 7 \mathrm{~B}-\mathrm{C} 16 \mathrm{~B}-\mathrm{C} 15 \mathrm{~B}$ & $2.4(4)$ \\
\hline $\mathrm{C} 8 \mathrm{~B}-\mathrm{C} 7 \mathrm{~B}-\mathrm{C} 16 \mathrm{~B}-\mathrm{C} 11 \mathrm{~B}$ & $-0.1(4)$ \\
\hline $\mathrm{C} 2 \mathrm{~B}-\mathrm{C} 7 \mathrm{~B}-\mathrm{C} 16 \mathrm{~B}-\mathrm{C} 11 \mathrm{~B}$ & $-176.4(2)$ \\
\hline $\mathrm{C} 23 \mathrm{~B}-\mathrm{C} 1 \mathrm{~B}-\mathrm{C} 17 \mathrm{~B}-\mathrm{C} 18 \mathrm{~B}$ & $103.0(3)$ \\
\hline $\mathrm{C} 2 \mathrm{~B}-\mathrm{C} 1 \mathrm{~B}-\mathrm{C} 17 \mathrm{~B}-\mathrm{C} 18 \mathrm{~B}$ & $-24.3(3)$ \\
\hline $\mathrm{C} 23 \mathrm{~B}-\mathrm{C} 1 \mathrm{~B}-\mathrm{C} 17 \mathrm{~B}-\mathrm{C} 22 \mathrm{~B}$ & $-73.6(3)$ \\
\hline $\mathrm{C} 2 \mathrm{~B}-\mathrm{C} 1 \mathrm{~B}-\mathrm{C} 17 \mathrm{~B}-\mathrm{C} 22 \mathrm{~B}$ & $159.1(2)$ \\
\hline $\mathrm{C} 22 \mathrm{~B}-\mathrm{C} 17 \mathrm{~B}-\mathrm{C} 18 \mathrm{~B}-\mathrm{C} 19 \mathrm{~B}$ & $0.1(4)$ \\
\hline $\mathrm{C} 1 \mathrm{~B}-\mathrm{C} 17 \mathrm{~B}-\mathrm{C} 18 \mathrm{~B}-\mathrm{C} 19 \mathrm{~B}$ & $-176.4(3)$ \\
\hline $\mathrm{C} 17 \mathrm{~B}-\mathrm{C} 18 \mathrm{~B}-\mathrm{C} 19 \mathrm{~B}-\mathrm{C} 20 \mathrm{~B}$ & $1.6(5)$ \\
\hline $\mathrm{C} 18 \mathrm{~B}-\mathrm{C} 19 \mathrm{~B}-\mathrm{C} 20 \mathrm{~B}-\mathrm{C} 21 \mathrm{~B}$ & $-1.8(5)$ \\
\hline $\mathrm{C} 19 \mathrm{~B}-\mathrm{C} 20 \mathrm{~B}-\mathrm{C} 21 \mathrm{~B}-\mathrm{C} 22 \mathrm{~B}$ & $0.3(5)$ \\
\hline $\mathrm{C} 20 \mathrm{~B}-\mathrm{C} 21 \mathrm{~B}-\mathrm{C} 22 \mathrm{~B}-\mathrm{C} 17 \mathrm{~B}$ & $1.4(5)$ \\
\hline $\mathrm{C} 18 \mathrm{~B}-\mathrm{C} 17 \mathrm{~B}-\mathrm{C} 22 \mathrm{~B}-\mathrm{C} 21 \mathrm{~B}$ & $-1.6(4)$ \\
\hline $\mathrm{C} 1 \mathrm{~B}-\mathrm{C} 17 \mathrm{~B}-\mathrm{C} 22 \mathrm{~B}-\mathrm{C} 21 \mathrm{~B}$ & $175.3(3)$ \\
\hline $\mathrm{C} 17 \mathrm{~B}-\mathrm{C} 1 \mathrm{~B}-\mathrm{C} 23 \mathrm{~B}-\mathrm{C} 24 \mathrm{~B}$ & $-46.9(3)$ \\
\hline $\mathrm{C} 2 \mathrm{~B}-\mathrm{C} 1 \mathrm{~B}-\mathrm{C} 23 \mathrm{~B}-\mathrm{C} 24 \mathrm{~B}$ & $83.4(3)$ \\
\hline $\mathrm{C} 17 \mathrm{~B}-\mathrm{C} 1 \mathrm{~B}-\mathrm{C} 23 \mathrm{~B}-\mathrm{C} 31 \mathrm{~B}$ & $133.8(2)$ \\
\hline $\mathrm{C} 2 \mathrm{~B}-\mathrm{C} 1 \mathrm{~B}-\mathrm{C} 23 \mathrm{~B}-\mathrm{C} 31 \mathrm{~B}$ & $-95.9(3)$ \\
\hline $\mathrm{C} 31 \mathrm{~B}-\mathrm{C} 23 \mathrm{~B}-\mathrm{C} 24 \mathrm{~B}-\mathrm{C} 25 \mathrm{~B}$ & $3.2(3)$ \\
\hline $\mathrm{C} 1 \mathrm{~B}-\mathrm{C} 23 \mathrm{~B}-\mathrm{C} 24 \mathrm{~B}-\mathrm{C} 25 \mathrm{~B}$ & $-176.1(2)$ \\
\hline $\mathrm{C} 23 \mathrm{~B}-\mathrm{C} 24 \mathrm{~B}-\mathrm{C} 25 \mathrm{~B}-\mathrm{C} 26 \mathrm{~B}$ & $-178.0(2)$ \\
\hline $\mathrm{C} 23 \mathrm{~B}-\mathrm{C} 24 \mathrm{~B}-\mathrm{C} 25 \mathrm{~B}-\mathrm{C} 30 \mathrm{~B}$ & $1.2(3)$ \\
\hline
\end{tabular}




$\begin{array}{ll}\mathrm{C} 30 \mathrm{~A}-\mathrm{C} 25 \mathrm{~A}-\mathrm{C} 26 \mathrm{~A}-\mathrm{C} 27 \mathrm{~A} & 1.8(4) \\ \mathrm{C} 24 \mathrm{~A}-\mathrm{C} 25 \mathrm{~A}-\mathrm{C} 26 \mathrm{~A}-\mathrm{C} 27 \mathrm{~A} & -179.1(3) \\ \mathrm{C} 25 \mathrm{~A}-\mathrm{C} 26 \mathrm{~A}-\mathrm{C} 27 \mathrm{~A}-\mathrm{C} 28 \mathrm{~A} & 0.8(4) \\ \mathrm{C} 25 \mathrm{~A}-\mathrm{C} 26 \mathrm{~A}-\mathrm{C} 27 \mathrm{~A}-\mathrm{Br} 1 \mathrm{~A} & -178.82(19) \\ \mathrm{C} 26 \mathrm{~A}-\mathrm{C} 27 \mathrm{~A}-\mathrm{C} 28 \mathrm{~A}-\mathrm{C} 29 \mathrm{~A} & -2.2(4) \\ \mathrm{Br} 1 \mathrm{~A}-\mathrm{C} 27 \mathrm{~A}-\mathrm{C} 28 \mathrm{~A}-\mathrm{C} 29 \mathrm{~A} & 177.4(2) \\ \mathrm{C} 27 \mathrm{~A}-\mathrm{C} 28 \mathrm{~A}-\mathrm{C} 29 \mathrm{~A}-\mathrm{C} 30 \mathrm{~A} & 0.9(4) \\ \mathrm{C} 31 \mathrm{~A}-\mathrm{N} 2 \mathrm{~A}-\mathrm{C} 30 \mathrm{~A}-\mathrm{C} 25 \mathrm{~A} & 1.5(4) \\ \mathrm{C} 31 \mathrm{~A}-\mathrm{N} 2 \mathrm{~A}-\mathrm{C} 30 \mathrm{~A}-\mathrm{C} 29 \mathrm{~A} & -178.4(2) \\ \mathrm{C} 26 \mathrm{~A}-\mathrm{C} 25 \mathrm{~A}-\mathrm{C} 30 \mathrm{~A}-\mathrm{N} 2 \mathrm{~A} & 177.1(2) \\ \mathrm{C} 24 \mathrm{~A}-\mathrm{C} 25 \mathrm{~A}-\mathrm{C} 30 \mathrm{~A}-\mathrm{N} 2 \mathrm{~A} & -2.1(4) \\ \mathrm{C} 26 \mathrm{~A}-\mathrm{C} 25 \mathrm{~A}-\mathrm{C} 30 \mathrm{~A}-\mathrm{C} 29 \mathrm{~A} & -3.0(3) \\ \mathrm{C} 24 \mathrm{~A}-\mathrm{C} 25 \mathrm{~A}-\mathrm{C} 30 \mathrm{~A}-\mathrm{C} 29 \mathrm{~A} & 177.9(2) \\ \mathrm{C} 28 \mathrm{~A}-\mathrm{C} 29 \mathrm{~A}-\mathrm{C} 30 \mathrm{~A}-\mathrm{N} 2 \mathrm{~A} & -178.4(3) \\ \mathrm{C} 28 \mathrm{~A}-\mathrm{C} 29 \mathrm{~A}-\mathrm{C} 30 \mathrm{~A}-\mathrm{C} 25 \mathrm{~A} & 1.7(4) \\ \mathrm{C} 30 \mathrm{~A}-\mathrm{N} 2 \mathrm{~A}-\mathrm{C} 31 \mathrm{~A}-\mathrm{O} 2 \mathrm{~A} & -178.0(2) \\ \mathrm{C} 30 \mathrm{~A}-\mathrm{N} 2 \mathrm{~A}-\mathrm{C} 31 \mathrm{~A}-\mathrm{C} 23 \mathrm{~A} & 1.3(4) \\ \mathrm{C} 32 \mathrm{~A}-\mathrm{O} 2 \mathrm{~A}-\mathrm{C} 31 \mathrm{~A}-\mathrm{N} 2 \mathrm{~A} & 14.6(4) \\ \mathrm{C} 32 \mathrm{~A}-\mathrm{O} 2 \mathrm{~A}-\mathrm{C} 31 \mathrm{~A}-\mathrm{C} 23 \mathrm{~A} & -164.7(3) \\ \mathrm{C} 24 \mathrm{~A}-\mathrm{C} 23 \mathrm{~A}-\mathrm{C} 31 \mathrm{~A}-\mathrm{N} 2 \mathrm{~A} & -3.5(4) \\ \mathrm{C} 1 \mathrm{~A}-\mathrm{C} 23 \mathrm{~A}-\mathrm{C} 31 \mathrm{~A}-\mathrm{N} 2 \mathrm{~A} & 176.6(2) \\ \mathrm{C} 24 \mathrm{~A}-\mathrm{C} 23 \mathrm{~A}-\mathrm{C} 31 \mathrm{~A}-\mathrm{O} 2 \mathrm{~A} & 175.9(2) \\ \mathrm{C} 1 \mathrm{~A}-\mathrm{C} 23 \mathrm{~A}-\mathrm{C} 31 \mathrm{~A}-\mathrm{O} 2 \mathrm{~A} & -4.1(3) \\ \mathrm{O} 3 \mathrm{~A}-\mathrm{C} 33 \mathrm{~A}-\mathrm{C} 34 \mathrm{~A}-\mathrm{C} 35 \mathrm{~A} & 6.7(4) \\ \mathrm{O} 4 \mathrm{~A}-\mathrm{C} 33 \mathrm{~A}-\mathrm{C} 34 \mathrm{~A}-\mathrm{C} 35 \mathrm{~A} & -173.8(3) \\ \mathrm{C} 33 \mathrm{~A}-\mathrm{C} 34 \mathrm{~A}-\mathrm{C} 35 \mathrm{~A}-\mathrm{C} 36 \mathrm{~A} & -176.1(2) \\ \mathrm{C} 34 \mathrm{~A}-\mathrm{C} 35 \mathrm{~A}-\mathrm{C} 36 \mathrm{~A}-\mathrm{O} 6 \mathrm{~A} & -30.1(4) \\ \mathrm{C} 34 \mathrm{~A}-\mathrm{C} 35 \mathrm{~A}-\mathrm{C} 36 \mathrm{~A}-\mathrm{O} 5 \mathrm{~A} & 148.2(3) \\ & \end{array}$

$\begin{array}{ll}\mathrm{C} 24 \mathrm{~B}-\mathrm{C} 25 \mathrm{~B}-\mathrm{C} 26 \mathrm{~B}-\mathrm{C} 27 \mathrm{~B} & -179.3(2) \\ \mathrm{C} 30 \mathrm{~B}-\mathrm{C} 25 \mathrm{~B}-\mathrm{C} 26 \mathrm{~B}-\mathrm{C} 27 \mathrm{~B} & 1.6(3) \\ \mathrm{C} 25 \mathrm{~B}-\mathrm{C} 26 \mathrm{~B}-\mathrm{C} 27 \mathrm{~B}-\mathrm{C} 28 \mathrm{~B} & 2.5(4) \\ \mathrm{C} 25 \mathrm{~B}-\mathrm{C} 26 \mathrm{~B}-\mathrm{C} 27 \mathrm{~B}-\mathrm{Br} 1 \mathrm{~B} & -176.19(17) \\ \mathrm{C} 26 \mathrm{~B}-\mathrm{C} 27 \mathrm{~B}-\mathrm{C} 28 \mathrm{~B}-\mathrm{C} 29 \mathrm{~B} & -3.7(4) \\ \mathrm{Br} 1 \mathrm{~B}-\mathrm{C} 27 \mathrm{~B}-\mathrm{C} 28 \mathrm{~B}-\mathrm{C} 29 \mathrm{~B} & 175.05(19) \\ \mathrm{C} 27 \mathrm{~B}-\mathrm{C} 28 \mathrm{~B}-\mathrm{C} 29 \mathrm{~B}-\mathrm{C} 30 \mathrm{~B} & 0.7(4) \\ \mathrm{C} 31 \mathrm{~B}-\mathrm{N} 2 \mathrm{~B}-\mathrm{C} 30 \mathrm{~B}-\mathrm{C} 29 \mathrm{~B} & -178.3(2) \\ \mathrm{C} 31 \mathrm{~B}-\mathrm{N} 2 \mathrm{~B}-\mathrm{C} 30 \mathrm{~B}-\mathrm{C} 25 \mathrm{~B} & 2.3(3) \\ \mathrm{C} 28 \mathrm{~B}-\mathrm{C} 29 \mathrm{~B}-\mathrm{C} 30 \mathrm{~B}-\mathrm{N} 2 \mathrm{~B} & -176.1(2) \\ \mathrm{C} 28 \mathrm{~B}-\mathrm{C} 29 \mathrm{~B}-\mathrm{C} 30 \mathrm{~B}-\mathrm{C} 25 \mathrm{~B} & 3.3(4) \\ \mathrm{C} 24 \mathrm{~B}-\mathrm{C} 25 \mathrm{~B}-\mathrm{C} 30 \mathrm{~B}-\mathrm{N} 2 \mathrm{~B} & -4.2(3) \\ \mathrm{C} 26 \mathrm{~B}-\mathrm{C} 25 \mathrm{~B}-\mathrm{C} 30 \mathrm{~B}-\mathrm{N} 2 \mathrm{~B} & 175.0(2) \\ \mathrm{C} 24 \mathrm{~B}-\mathrm{C} 25 \mathrm{~B}-\mathrm{C} 30 \mathrm{~B}-\mathrm{C} 29 \mathrm{~B} & 176.4(2) \\ \mathrm{C} 26 \mathrm{~B}-\mathrm{C} 25 \mathrm{~B}-\mathrm{C} 30 \mathrm{~B}-\mathrm{C} 29 \mathrm{~B} & -4.4(3) \\ \mathrm{C} 30 \mathrm{~B}-\mathrm{N} 2 \mathrm{~B}-\mathrm{C} 31 \mathrm{~B}-\mathrm{O} 2 \mathrm{~B} & -176.9(2) \\ \mathrm{C} 30 \mathrm{~B}-\mathrm{N} 2 \mathrm{~B}-\mathrm{C} 31 \mathrm{~B}-\mathrm{C} 23 \mathrm{~B} & 2.6(4) \\ \mathrm{C} 32 \mathrm{~B}-\mathrm{O} 2 \mathrm{~B}-\mathrm{C} 31 \mathrm{~B}-\mathrm{N} 2 \mathrm{~B} & 10.6(4) \\ \mathrm{C} 32 \mathrm{~B}-\mathrm{O} 2 \mathrm{~B}-\mathrm{C} 31 \mathrm{~B}-\mathrm{C} 23 \mathrm{~B} & -169.0(3) \\ \mathrm{C} 24 \mathrm{~B}-\mathrm{C} 23 \mathrm{~B}-\mathrm{C} 31 \mathrm{~B}-\mathrm{N} 2 \mathrm{~B} & -5.4(4) \\ \mathrm{C} 1 \mathrm{~B}-\mathrm{C} 23 \mathrm{~B}-\mathrm{C} 31 \mathrm{~B}-\mathrm{N} 2 \mathrm{~B} & 173.9(2) \\ \mathrm{C} 24 \mathrm{~B}-\mathrm{C} 23 \mathrm{~B}-\mathrm{C} 31 \mathrm{~B}-\mathrm{O} 2 \mathrm{~B} & 174.1(2) \\ \mathrm{C} 1 \mathrm{~B}-\mathrm{C} 23 \mathrm{~B}-\mathrm{C} 31 \mathrm{~B}-\mathrm{O} 2 \mathrm{~B} & -6.6(3) \\ \mathrm{O} 4 \mathrm{~B}-\mathrm{C} 33 \mathrm{~B}-\mathrm{C} 34 \mathrm{~B}-\mathrm{C} 35 \mathrm{~B} & -178.3(3) \\ \mathrm{O} 3 \mathrm{~B}-\mathrm{C} 33 \mathrm{~B}-\mathrm{C} 34 \mathrm{~B}-\mathrm{C} 35 \mathrm{~B} & 3.8(4) \\ \mathrm{C} 33 \mathrm{~B}-\mathrm{C} 34 \mathrm{~B}-\mathrm{C} 35 \mathrm{~B}-\mathrm{C} 36 \mathrm{~B} & -172.7(2) \\ \mathrm{C} 34 \mathrm{~B}-\mathrm{C} 35 \mathrm{~B}-\mathrm{C} 36 \mathrm{~B}-\mathrm{O} 5 \mathrm{~B} & 161.3(3) \\ \mathrm{C} 34 \mathrm{~B}-\mathrm{C} 35 \mathrm{~B}-\mathrm{C} 36 \mathrm{~B}-\mathrm{O} 6 \mathrm{~B} & -16.6(4) \\ & \end{array}$

Hydrogen-bond geometry $\left(A,{ }^{\circ}\right)$

\begin{tabular}{lllll}
\hline$D-\mathrm{H} \cdots A$ & $D-\mathrm{H}$ & $\mathrm{H} \cdots A$ & $D \cdots A$ & $D-\mathrm{H} \cdots A$ \\
\hline $\mathrm{O} 1 A-\mathrm{H} 1 A B \cdots \mathrm{O} 4 A$ & $0.86(4)$ & $1.88(4)$ & $2.699(3)$ & $159(4)$ \\
$\mathrm{O} 5 A-\mathrm{H} 5 A \cdots \mathrm{O} 4 B$ & $1.04(4)$ & $1.58(4)$ & $2.603(3)$ & $169(4)$ \\
$\mathrm{N} 1 A-\mathrm{H} 1 A N \cdots \mathrm{O} 3 A$ & $0.98(4)$ & $1.68(4)$ & $2.641(3)$ & $163(3)$ \\
$\mathrm{C} 1 A-\mathrm{H} 1 A \cdots \mathrm{O} 2 A$ & 1.00 & 2.23 & $2.767(3)$ & 112 \\
$\mathrm{C} 3 A-\mathrm{H} 3 A B \cdots \mathrm{Br} 1 B^{\mathrm{i}}$ & 0.99 & 3.01 & $3.850(3)$ & 143 \\
$\mathrm{C} 5 A-\mathrm{H} 5 A A \cdots \mathrm{O} 1 A^{\mathrm{ii}}$ & 0.98 & 2.58 & $3.501(3)$ & 157 \\
$\mathrm{C} 5 A-\mathrm{H} 5 A C \cdots \mathrm{Br} 1 B^{\mathrm{i}}$ & 0.98 & 2.82 & $3.612(3)$ & 139 \\
$\mathrm{C} 26 A-\mathrm{H} 26 A \cdots \mathrm{O} 5 B^{\mathrm{iii}}$ & 0.95 & 2.54 & $3.459(4)$ & 163 \\
$\mathrm{C} 34 A-\mathrm{H} 34 A \cdots \mathrm{O} 5 B^{\mathrm{iii}}$ & 0.95 & 2.52 & $3.379(3)$ & 151 \\
$\mathrm{O} 1 B-\mathrm{H} 1 B \cdots \mathrm{O} 4 B^{\mathrm{iv}}$ & $0.89(4)$ & $1.88(4)$ & $2.741(3)$ & $160(4)$ \\
$\mathrm{O} 6 B-\mathrm{H} 6 B \cdots \mathrm{O} 4 A^{\mathrm{v}}$ & $0.86(5)$ & $1.80(5)$ & $2.625(3)$ & $159(4)$ \\
$\mathrm{N} 1 B-\mathrm{H} 1 B N \cdots \mathrm{O} 3 B^{\mathrm{iv}}$ & $0.85(4)$ & $1.83(4)$ & $2.632(3)$ & $158(4)$ \\
$\mathrm{C} 5 B-\mathrm{H} 5 B A \cdots \mathrm{O} 1 B^{\mathrm{vi}}$ & 0.98 & 2.42 & $3.337(4)$ & 156
\end{tabular}




$\begin{array}{llllr}\mathrm{C} 5 B-\mathrm{H} 5 B B \cdots \mathrm{Br} 1 A & 0.98 & 2.88 & 3.468(3) & 119 \\ \mathrm{C} 5 B-\mathrm{H} 5 B B \cdots \mathrm{O} 6 A & 0.98 & 2.42 & 3.287(4) & 148\end{array}$

Symmetry codes: (i) $x+1, y, z+1$; (ii) $-x+2, y+1 / 2,-z+1$; (iii) $x, y-1, z$; (iv) $-x+1, y-1 / 2,-z$; (v) $x, y+1, z$; (vi) $-x+1, y+1 / 2,-z$.

3-Benzyl-6-bromo-2-methoxyquinoline (3)

\section{Crystal data}

$\mathrm{C}_{17} \mathrm{H}_{14} \mathrm{BrNO}$

$M_{r}=328.20$

Orthorhombic, $P 2{ }_{1} 2_{1} 2_{1}$

$a=4.3606$ (6) $\AA$

$b=10.820(2) \AA$

$c=29.886(11) \AA$

$V=1410.1(6) \AA^{3}$

$Z=4$

$F(000)=664$

\section{Data collection}

\section{Bruker D8 Quest}

diffractometer with PhotonII charge-integrating pixel array detector (CPAD)

Radiation source: fine focus sealed tube X-ray source

Triumph curved graphite crystal monochromator

Detector resolution: 7.4074 pixels $\mathrm{mm}^{-1}$

$\omega$ and phi scans

\section{Refinement}

Refinement on $F^{2}$

Least-squares matrix: full

$R\left[F^{2}>2 \sigma\left(F^{2}\right)\right]=0.023$

$w R\left(F^{2}\right)=0.057$

$S=1.05$

5125 reflections

196 parameters

0 restraints

Primary atom site location: structure-invariant direct methods

Secondary atom site location: difference Fourier map
$D_{\mathrm{x}}=1.546 \mathrm{Mg} \mathrm{m}^{-3}$

Mo $K \alpha$ radiation, $\lambda=0.71073 \AA$

Cell parameters from 9920 reflections

$\theta=2.3-32.7^{\circ}$

$\mu=2.91 \mathrm{~mm}^{-1}$

$T=150 \mathrm{~K}$

Needle, colourless

$0.41 \times 0.06 \times 0.05 \mathrm{~mm}$

Absorption correction: multi-scan

(SADABS2016; Krause et al., 2015)

$T_{\min }=0.658, T_{\max }=0.747$

28030 measured reflections

5125 independent reflections

4504 reflections with $I>2 \sigma(I)$

$R_{\text {int }}=0.037$

$\theta_{\max }=33.1^{\circ}, \theta_{\min }=2.8^{\circ}$

$h=-6 \rightarrow 5$

$k=-16 \rightarrow 16$

$l=-45 \rightarrow 40$

Hydrogen site location: inferred from neighbouring sites

Only $\mathrm{H}$-atom displacement parameters refined

$w=1 /\left[\sigma^{2}\left(F_{0}^{2}\right)+(0.0308 P)^{2}\right]$

where $P=\left(F_{\mathrm{o}}^{2}+2 F_{\mathrm{c}}^{2}\right) / 3$

$(\Delta / \sigma)_{\max }<0.001$

$\Delta \rho_{\max }=0.28 \mathrm{e} \AA^{-3}$

$\Delta \rho_{\min }=-0.38$ e $\AA^{-3}$

Absolute structure: Flack $x$ determined using 1685 quotients [(I+)-(I-)]/[(I+)+(I-)] (Parsons et al., 2013)

Absolute structure parameter: -0.011 (3)

Special details

Geometry. All esds (except the esd in the dihedral angle between two 1.s. planes) are estimated using the full covariance matrix. The cell esds are taken into account individually in the estimation of esds in distances, angles and torsion angles; correlations between esds in cell parameters are only used when they are defined by crystal symmetry. An approximate (isotropic) treatment of cell esds is used for estimating esds involving l.s. planes.

Fractional atomic coordinates and isotropic or equivalent isotropic displacement parameters $\left(\AA^{2}\right)$

\begin{tabular}{lllll}
\hline & $x$ & $y$ & $z$ & $U_{\text {iso }} * / U_{\text {eq }}$ \\
\hline Br1 & $0.33234(5)$ & $1.09654(2)$ & $0.10466(2)$ & $0.03579(6)$
\end{tabular}




$\begin{array}{lllll}\text { O1 } & 0.9170(3) & 0.38638(11) & 0.08083(4) & 0.0283(3) \\ \text { N1 } & 0.6613(3) & 0.56540(12) & 0.06323(4) & 0.0214(2) \\ \text { C1 } & 0.8442(4) & 0.50555(14) & 0.09018(5) & 0.0214(3) \\ \text { C2 } & 0.9779(3) & 0.55404(15) & 0.13019(6) & 0.0206(3) \\ \text { C3 } & 0.9145(3) & 0.67422(16) & 0.14000(6) & 0.0212(3) \\ \text { H3 } & 1.003058 & 0.711254 & 0.165763 & 0.027(5)^{*} \\ \text { C4 } & 0.7166(3) & 0.74501(15) & 0.11205(5) & 0.0191(3) \\ \text { C5 } & 0.6405(4) & 0.86982(15) & 0.12085(6) & 0.0227(3) \\ \text { H5 } & 0.724285 & 0.911329 & 0.146058 & 0.028(5)^{*} \\ \text { C6 } & 0.4437(4) & 0.92991(14) & 0.09234(6) & 0.0236(3) \\ \text { C7 } & 0.3168(4) & 0.87254(15) & 0.05471(6) & 0.0247(3) \\ \text { H7 } & 0.181534 & 0.916658 & 0.035583 & 0.034(6)^{*} \\ \text { C8 } & 0.3902(4) & 0.75158(16) & 0.04576(6) & 0.0229(3) \\ \text { H8 } & 0.303981 & 0.711802 & 0.020373 & 0.031(5)^{*} \\ \text { C9 } & 0.5927(3) & 0.68571(14) & 0.07392(5) & 0.0192(3) \\ \text { C10 } & 0.7927(6) & 0.33696(18) & 0.04034(7) & 0.0386(5) \\ \text { H10A } & 0.873753 & 0.383151 & 0.014732 & 0.045(7)^{*} \\ \text { H10B } & 0.568653 & 0.344120 & 0.040923 & 0.051(8)^{*} \\ \text { H10C } & 0.850322 & 0.249746 & 0.037603 & 0.048(7)^{*} \\ \text { C11 } & 1.1699(4) & 0.47323(16) & 0.16067(6) & 0.0240(3) \\ \text { H11A } & 1.325806 & 0.524748 & 0.175900 & 0.034(6)^{*} \\ \text { H11B } & 1.278720 & 0.410507 & 0.142523 & 0.049(7)^{*} \\ \text { C12 } & 0.9739(3) & 0.40881(15) & 0.19546(5) & 0.0205(3) \\ \text { C13 } & 0.8353(4) & 0.29555(14) & 0.18642(6) & 0.0246(3) \\ \text { H13 } & 0.866613 & 0.256921 & 0.158245 & 0.022(5)^{*} \\ \text { C14 } & 0.6512(4) & 0.23861(15) & 0.21838(6) & 0.0294(3) \\ \text { H14 } & 0.556300 & 0.161713 & 0.211782 & 0.055(8)^{*} \\ \text { C15 } & 0.6057(4) & 0.29308(18) & 0.25961(7) & 0.0310(4) \\ \text { H15 } & 0.481362 & 0.253555 & 0.281437 & 0.035(6)^{*} \\ \text { C16 } & 0.7424(4) & 0.40577(18) & 0.26897(6) & 0.0302(4) \\ \text { H16 } & 0.711861 & 0.443543 & 0.297316 & 0.035(6)^{*} \\ \text { C17 } & 0.9236(4) & 0.46362(16) & 0.23709(6) & 0.0267(4) \\ \text { H17 } & 1.014500 & 0.541330 & 0.243665 & 0.051(7)^{*} \\ & & & & \end{array}$

Atomic displacement parameters $\left(\AA^{2}\right)$

\begin{tabular}{lllllll}
\hline & $U^{11}$ & $U^{22}$ & $U^{33}$ & $U^{12}$ & $U^{13}$ & $U^{23}$ \\
\hline Br1 & $0.04286(10)$ & $0.02454(8)$ & $0.03998(11)$ & $0.00613(8)$ & $-0.00053(9)$ & $-0.00279(8)$ \\
O1 & $0.0374(6)$ & $0.0241(6)$ & $0.0233(6)$ & $0.0055(5)$ & $-0.0016(5)$ & $-0.0014(5)$ \\
N1 & $0.0230(6)$ & $0.0238(6)$ & $0.0175(6)$ & $0.0004(5)$ & $-0.0001(6)$ & $-0.0002(5)$ \\
C1 & $0.0220(6)$ & $0.0235(7)$ & $0.0188(7)$ & $0.0006(6)$ & $0.0033(6)$ & $0.0004(5)$ \\
C2 & $0.0155(6)$ & $0.0279(7)$ & $0.0185(7)$ & $-0.0020(5)$ & $0.0015(5)$ & $0.0044(6)$ \\
C3 & $0.0180(6)$ & $0.0278(8)$ & $0.0178(7)$ & $-0.0033(5)$ & $0.0000(5)$ & $0.0014(6)$ \\
C4 & $0.0177(6)$ & $0.0243(7)$ & $0.0153(7)$ & $-0.0016(5)$ & $0.0025(5)$ & $0.0010(5)$ \\
C5 & $0.0237(7)$ & $0.0254(7)$ & $0.0190(7)$ & $-0.0023(6)$ & $0.0022(6)$ & $-0.0016(5)$ \\
C6 & $0.0244(7)$ & $0.0221(7)$ & $0.0242(8)$ & $0.0006(5)$ & $0.0061(6)$ & $0.0006(6)$ \\
C7 & $0.0241(7)$ & $0.0286(7)$ & $0.0215(8)$ & $0.0026(6)$ & $0.0024(7)$ & $0.0049(6)$ \\
C8 & $0.0229(7)$ & $0.0295(8)$ & $0.0164(7)$ & $-0.0005(6)$ & $-0.0005(5)$ & $0.0004(6)$
\end{tabular}


supporting information

\begin{tabular}{lllllll} 
C9 & $0.0182(6)$ & $0.0234(7)$ & $0.0159(7)$ & $-0.0011(5)$ & $0.0030(5)$ & $0.0017(6)$ \\
C10 & $0.0623(13)$ & $0.0286(9)$ & $0.0250(9)$ & $0.0078(9)$ & $-0.0066(9)$ & $-0.0059(7)$ \\
C11 & $0.0165(6)$ & $0.0319(8)$ & $0.0237(8)$ & $0.0022(6)$ & $-0.0013(7)$ & $0.0036(6)$ \\
C12 & $0.0169(6)$ & $0.0242(7)$ & $0.0205(7)$ & $0.0047(6)$ & $-0.0025(5)$ & $0.0039(6)$ \\
C13 & $0.0252(7)$ & $0.0236(7)$ & $0.0251(8)$ & $0.0041(7)$ & $-0.0031(7)$ & $0.0005(6)$ \\
C14 & $0.0300(8)$ & $0.0236(7)$ & $0.0345(9)$ & $-0.0001(7)$ & $-0.0037(8)$ & $0.0073(7)$ \\
C15 & $0.0275(8)$ & $0.0345(9)$ & $0.0311(9)$ & $0.0036(7)$ & $0.0018(7)$ & $0.0131(7)$ \\
C16 & $0.0335(8)$ & $0.0354(9)$ & $0.0216(8)$ & $0.0077(7)$ & $0.0013(6)$ & $0.0021(7)$ \\
C17 & $0.0296(8)$ & $0.0260(8)$ & $0.0246(9)$ & $0.0011(6)$ & $-0.0038(6)$ & $-0.0011(7)$ \\
\hline
\end{tabular}

Geometric parameters $\left(\AA,{ }^{\circ}\right)$

\begin{tabular}{|c|c|c|c|}
\hline $\mathrm{Br} 1-\mathrm{C} 6$ & $1.9030(16)$ & $\mathrm{C} 8-\mathrm{H} 8$ & 0.9500 \\
\hline $\mathrm{O} 1-\mathrm{C} 1$ & $1.3570(19)$ & $\mathrm{C} 10-\mathrm{H} 10 \mathrm{~A}$ & 0.9800 \\
\hline $\mathrm{O} 1-\mathrm{C} 10$ & $1.430(2)$ & $\mathrm{C} 10-\mathrm{H} 10 \mathrm{~B}$ & 0.9800 \\
\hline $\mathrm{N} 1-\mathrm{C} 1$ & $1.305(2)$ & $\mathrm{C} 10-\mathrm{H} 10 \mathrm{C}$ & 0.9800 \\
\hline $\mathrm{N} 1-\mathrm{C} 9$ & $1.373(2)$ & $\mathrm{C} 11-\mathrm{C} 12$ & $1.516(2)$ \\
\hline $\mathrm{C} 1-\mathrm{C} 2$ & $1.430(2)$ & $\mathrm{C} 11-\mathrm{H} 11 \mathrm{~A}$ & 0.9900 \\
\hline $\mathrm{C} 2-\mathrm{C} 3$ & $1.361(2)$ & $\mathrm{C} 11-\mathrm{H} 11 \mathrm{~B}$ & 0.9900 \\
\hline $\mathrm{C} 2-\mathrm{C} 11$ & $1.515(2)$ & $\mathrm{C} 12-\mathrm{C} 13$ & $1.393(2)$ \\
\hline $\mathrm{C} 3-\mathrm{C} 4$ & $1.424(2)$ & $\mathrm{C} 12-\mathrm{C} 17$ & $1.396(2)$ \\
\hline $\mathrm{C} 3-\mathrm{H} 3$ & 0.9500 & $\mathrm{C} 13-\mathrm{C} 14$ & $1.391(3)$ \\
\hline $\mathrm{C} 4-\mathrm{C} 9$ & $1.415(2)$ & $\mathrm{C} 13-\mathrm{H} 13$ & 0.9500 \\
\hline $\mathrm{C} 4-\mathrm{C} 5$ & $1.415(2)$ & $\mathrm{C} 14-\mathrm{C} 15$ & $1.380(3)$ \\
\hline $\mathrm{C} 5-\mathrm{C} 6$ & $1.373(2)$ & C14-H14 & 0.9500 \\
\hline $\mathrm{C} 5-\mathrm{H} 5$ & 0.9500 & $\mathrm{C} 15-\mathrm{C} 16$ & $1.386(3)$ \\
\hline $\mathrm{C} 6-\mathrm{C} 7$ & $1.399(3)$ & $\mathrm{C} 15-\mathrm{H} 15$ & 0.9500 \\
\hline $\mathrm{C} 7-\mathrm{C} 8$ & $1.374(2)$ & $\mathrm{C} 16-\mathrm{C} 17$ & $1.387(3)$ \\
\hline $\mathrm{C} 7-\mathrm{H} 7$ & 0.9500 & $\mathrm{C} 16-\mathrm{H} 16$ & 0.9500 \\
\hline $\mathrm{C} 8-\mathrm{C} 9$ & $1.413(2)$ & $\mathrm{C} 17-\mathrm{H} 17$ & 0.9500 \\
\hline $\mathrm{C} 1-\mathrm{O} 1-\mathrm{C} 10$ & $116.15(14)$ & $\mathrm{O} 1-\mathrm{C} 10-\mathrm{H} 10 \mathrm{~B}$ & 109.5 \\
\hline $\mathrm{C} 1-\mathrm{N} 1-\mathrm{C} 9$ & $117.37(14)$ & $\mathrm{H} 10 \mathrm{~A}-\mathrm{C} 10-\mathrm{H} 10 \mathrm{~B}$ & 109.5 \\
\hline $\mathrm{N} 1-\mathrm{C} 1-\mathrm{O} 1$ & $119.16(14)$ & $\mathrm{O} 1-\mathrm{C} 10-\mathrm{H} 10 \mathrm{C}$ & 109.5 \\
\hline $\mathrm{N} 1-\mathrm{C} 1-\mathrm{C} 2$ & $125.67(15)$ & $\mathrm{H} 10 \mathrm{~A}-\mathrm{C} 10-\mathrm{H} 10 \mathrm{C}$ & 109.5 \\
\hline $\mathrm{O} 1-\mathrm{C} 1-\mathrm{C} 2$ & $115.16(14)$ & $\mathrm{H} 10 \mathrm{~B}-\mathrm{C} 10-\mathrm{H} 10 \mathrm{C}$ & 109.5 \\
\hline $\mathrm{C} 3-\mathrm{C} 2-\mathrm{C} 1$ & $116.59(15)$ & $\mathrm{C} 2-\mathrm{C} 11-\mathrm{C} 12$ & $111.49(13)$ \\
\hline $\mathrm{C} 3-\mathrm{C} 2-\mathrm{C} 11$ & $122.27(16)$ & $\mathrm{C} 2-\mathrm{C} 11-\mathrm{H} 11 \mathrm{~A}$ & 109.3 \\
\hline $\mathrm{C} 1-\mathrm{C} 2-\mathrm{C} 11$ & $121.09(15)$ & $\mathrm{C} 12-\mathrm{C} 11-\mathrm{H} 11 \mathrm{~A}$ & 109.3 \\
\hline $\mathrm{C} 2-\mathrm{C} 3-\mathrm{C} 4$ & $120.69(15)$ & $\mathrm{C} 2-\mathrm{C} 11-\mathrm{H} 11 \mathrm{~B}$ & 109.3 \\
\hline $\mathrm{C} 2-\mathrm{C} 3-\mathrm{H} 3$ & 119.7 & $\mathrm{C} 12-\mathrm{C} 11-\mathrm{H} 11 \mathrm{~B}$ & 109.3 \\
\hline $\mathrm{C} 4-\mathrm{C} 3-\mathrm{H} 3$ & 119.7 & $\mathrm{H} 11 \mathrm{~A}-\mathrm{C} 11-\mathrm{H} 11 \mathrm{~B}$ & 108.0 \\
\hline $\mathrm{C} 9-\mathrm{C} 4-\mathrm{C} 5$ & $119.53(14)$ & $\mathrm{C} 13-\mathrm{C} 12-\mathrm{C} 17$ & $118.60(15)$ \\
\hline $\mathrm{C} 9-\mathrm{C} 4-\mathrm{C} 3$ & $117.37(15)$ & $\mathrm{C} 13-\mathrm{C} 12-\mathrm{C} 11$ & $121.08(15)$ \\
\hline $\mathrm{C} 5-\mathrm{C} 4-\mathrm{C} 3$ & $123.10(15)$ & $\mathrm{C} 17-\mathrm{C} 12-\mathrm{C} 11$ & $120.30(15)$ \\
\hline $\mathrm{C} 6-\mathrm{C} 5-\mathrm{C} 4$ & $118.88(15)$ & $\mathrm{C} 14-\mathrm{C} 13-\mathrm{C} 12$ & $120.44(16)$ \\
\hline $\mathrm{C} 6-\mathrm{C} 5-\mathrm{H} 5$ & 120.6 & $\mathrm{C} 14-\mathrm{C} 13-\mathrm{H} 13$ & 119.8 \\
\hline $\mathrm{C} 4-\mathrm{C} 5-\mathrm{H} 5$ & 120.6 & $\mathrm{C} 12-\mathrm{C} 13-\mathrm{H} 13$ & 119.8 \\
\hline
\end{tabular}




\begin{tabular}{|c|c|c|c|}
\hline $\mathrm{C} 5-\mathrm{C} 6-\mathrm{C} 7$ & $122.42(15)$ & $\mathrm{C} 15-\mathrm{C} 14-\mathrm{C} 13$ & $120.45(16)$ \\
\hline $\mathrm{C} 5-\mathrm{C} 6-\mathrm{Br} 1$ & $119.21(13)$ & $\mathrm{C} 15-\mathrm{C} 14-\mathrm{H} 14$ & 119.8 \\
\hline $\mathrm{C} 7-\mathrm{C} 6-\mathrm{Br} 1$ & $118.36(12)$ & $\mathrm{C} 13-\mathrm{C} 14-\mathrm{H} 14$ & 119.8 \\
\hline $\mathrm{C} 8-\mathrm{C} 7-\mathrm{C} 6$ & $119.15(16)$ & $\mathrm{C} 14-\mathrm{C} 15-\mathrm{C} 16$ & $119.61(17)$ \\
\hline $\mathrm{C} 8-\mathrm{C} 7-\mathrm{H} 7$ & 120.4 & $\mathrm{C} 14-\mathrm{C} 15-\mathrm{H} 15$ & 120.2 \\
\hline $\mathrm{C} 6-\mathrm{C} 7-\mathrm{H} 7$ & 120.4 & $\mathrm{C} 16-\mathrm{C} 15-\mathrm{H} 15$ & 120.2 \\
\hline $\mathrm{C} 7-\mathrm{C} 8-\mathrm{C} 9$ & $120.69(16)$ & $\mathrm{C} 15-\mathrm{C} 16-\mathrm{C} 17$ & $120.23(17)$ \\
\hline $\mathrm{C} 7-\mathrm{C} 8-\mathrm{H} 8$ & 119.7 & $\mathrm{C} 15-\mathrm{C} 16-\mathrm{H} 16$ & 119.9 \\
\hline $\mathrm{C} 9-\mathrm{C} 8-\mathrm{H} 8$ & 119.7 & $\mathrm{C} 17-\mathrm{C} 16-\mathrm{H} 16$ & 119.9 \\
\hline $\mathrm{N} 1-\mathrm{C} 9-\mathrm{C} 8$ & $118.41(15)$ & $\mathrm{C} 16-\mathrm{C} 17-\mathrm{C} 12$ & $120.66(16)$ \\
\hline $\mathrm{N} 1-\mathrm{C} 9-\mathrm{C} 4$ & $122.27(14)$ & $\mathrm{C} 16-\mathrm{C} 17-\mathrm{H} 17$ & 119.7 \\
\hline $\mathrm{C} 8-\mathrm{C} 9-\mathrm{C} 4$ & $119.32(15)$ & $\mathrm{C} 12-\mathrm{C} 17-\mathrm{H} 17$ & 119.7 \\
\hline $\mathrm{O} 1-\mathrm{C} 10-\mathrm{H} 10 \mathrm{~A}$ & 109.5 & & \\
\hline $\mathrm{C} 9-\mathrm{N} 1-\mathrm{C} 1-\mathrm{O} 1$ & $179.86(14)$ & $\mathrm{C} 1-\mathrm{N} 1-\mathrm{C} 9-\mathrm{C} 4$ & $1.0(2)$ \\
\hline $\mathrm{C} 9-\mathrm{N} 1-\mathrm{C} 1-\mathrm{C} 2$ & $0.5(2)$ & $\mathrm{C} 7-\mathrm{C} 8-\mathrm{C} 9-\mathrm{N} 1$ & $-179.29(15)$ \\
\hline $\mathrm{C} 10-\mathrm{O} 1-\mathrm{C} 1-\mathrm{N} 1$ & $2.2(2)$ & $\mathrm{C} 7-\mathrm{C} 8-\mathrm{C} 9-\mathrm{C} 4$ & $1.0(2)$ \\
\hline $\mathrm{C} 10-\mathrm{O} 1-\mathrm{C} 1-\mathrm{C} 2$ & $-178.34(16)$ & $\mathrm{C} 5-\mathrm{C} 4-\mathrm{C} 9-\mathrm{N} 1$ & $179.02(14)$ \\
\hline $\mathrm{N} 1-\mathrm{C} 1-\mathrm{C} 2-\mathrm{C} 3$ & $-2.0(2)$ & $\mathrm{C} 3-\mathrm{C} 4-\mathrm{C} 9-\mathrm{N} 1$ & $-1.0(2)$ \\
\hline $\mathrm{O} 1-\mathrm{C} 1-\mathrm{C} 2-\mathrm{C} 3$ & $178.66(14)$ & $\mathrm{C} 5-\mathrm{C} 4-\mathrm{C} 9-\mathrm{C} 8$ & $-1.2(2)$ \\
\hline $\mathrm{N} 1-\mathrm{C} 1-\mathrm{C} 2-\mathrm{C} 11$ & $175.74(16)$ & $\mathrm{C} 3-\mathrm{C} 4-\mathrm{C} 9-\mathrm{C} 8$ & $178.69(14)$ \\
\hline $\mathrm{O} 1-\mathrm{C} 1-\mathrm{C} 2-\mathrm{C} 11$ & $-3.6(2)$ & $\mathrm{C} 3-\mathrm{C} 2-\mathrm{C} 11-\mathrm{C} 12$ & $88.62(19)$ \\
\hline $\mathrm{C} 1-\mathrm{C} 2-\mathrm{C} 3-\mathrm{C} 4$ & $1.9(2)$ & $\mathrm{C} 1-\mathrm{C} 2-\mathrm{C} 11-\mathrm{C} 12$ & $-88.96(19)$ \\
\hline $\mathrm{C} 11-\mathrm{C} 2-\mathrm{C} 3-\mathrm{C} 4$ & $-175.82(14)$ & $\mathrm{C} 2-\mathrm{C} 11-\mathrm{C} 12-\mathrm{C} 13$ & $87.29(18)$ \\
\hline $\mathrm{C} 2-\mathrm{C} 3-\mathrm{C} 4-\mathrm{C} 9$ & $-0.5(2)$ & $\mathrm{C} 2-\mathrm{C} 11-\mathrm{C} 12-\mathrm{C} 17$ & $-91.38(18)$ \\
\hline $\mathrm{C} 2-\mathrm{C} 3-\mathrm{C} 4-\mathrm{C} 5$ & $179.43(14)$ & $\mathrm{C} 17-\mathrm{C} 12-\mathrm{C} 13-\mathrm{C} 14$ & $-0.1(2)$ \\
\hline $\mathrm{C} 9-\mathrm{C} 4-\mathrm{C} 5-\mathrm{C} 6$ & $1.0(2)$ & $\mathrm{C} 11-\mathrm{C} 12-\mathrm{C} 13-\mathrm{C} 14$ & $-178.79(15)$ \\
\hline $\mathrm{C} 3-\mathrm{C} 4-\mathrm{C} 5-\mathrm{C} 6$ & $-178.96(15)$ & $\mathrm{C} 12-\mathrm{C} 13-\mathrm{C} 14-\mathrm{C} 15$ & $-0.6(3)$ \\
\hline $\mathrm{C} 4-\mathrm{C} 5-\mathrm{C} 6-\mathrm{C} 7$ & $-0.4(3)$ & $\mathrm{C} 13-\mathrm{C} 14-\mathrm{C} 15-\mathrm{C} 16$ & $0.6(3)$ \\
\hline $\mathrm{C} 4-\mathrm{C} 5-\mathrm{C} 6-\mathrm{Br} 1$ & $178.51(11)$ & $\mathrm{C} 14-\mathrm{C} 15-\mathrm{C} 16-\mathrm{C} 17$ & $0.1(3)$ \\
\hline $\mathrm{C} 5-\mathrm{C} 6-\mathrm{C} 7-\mathrm{C} 8$ & $0.1(3)$ & $\mathrm{C} 15-\mathrm{C} 16-\mathrm{C} 17-\mathrm{C} 12$ & $-0.7(3)$ \\
\hline $\mathrm{Br} 1-\mathrm{C} 6-\mathrm{C} 7-\mathrm{C} 8$ & $-178.82(13)$ & $\mathrm{C} 13-\mathrm{C} 12-\mathrm{C} 17-\mathrm{C} 16$ & $0.7(2)$ \\
\hline $\mathrm{C} 6-\mathrm{C} 7-\mathrm{C} 8-\mathrm{C} 9$ & $-0.4(2)$ & $\mathrm{C} 11-\mathrm{C} 12-\mathrm{C} 17-\mathrm{C} 16$ & $179.44(15)$ \\
\hline $\mathrm{C} 1-\mathrm{N} 1-\mathrm{C} 9-\mathrm{C} 8$ & $-178.70(15)$ & & \\
\hline
\end{tabular}

[4-(6-Bromo-2-methoxyquinolin-3-yl)-3-hydroxy-3-(naphthalen-1-yl)-4-phenylbutyl]dimethylazanium benzoate hydrate (4a)

\section{Crystal data}

$\mathrm{C}_{32} \mathrm{H}_{32} \mathrm{BrN}_{2} \mathrm{O}_{2}^{+} \cdot \mathrm{C}_{7} \mathrm{H}_{5} \mathrm{O}_{2} \cdot \cdot 1.166 \mathrm{H}_{2} \mathrm{O}$

$M_{r}=698.70$

Monoclinic, $P 2_{1}$

$a=12.6384(5) \AA$

$b=7.9259(3) \AA$

$c=17.5249(8) \AA$

$\beta=99.8450(17)^{\circ}$

$V=1729.63(12) \AA^{3}$

$Z=2$
$F(000)=727$

$D_{\mathrm{x}}=1.342 \mathrm{Mg} \mathrm{m}^{-3}$

Mo $K \alpha$ radiation, $\lambda=0.71073 \AA$

Cell parameters from 9883 reflections

$\theta=2.4-31.0^{\circ}$

$\mu=1.24 \mathrm{~mm}^{-1}$

$T=150 \mathrm{~K}$

Rod, colourless

$0.55 \times 0.21 \times 0.13 \mathrm{~mm}$ 


\section{Data collection}

Bruker D8 Quest diffractometer with PhotonII charge-integrating pixel array detector (CPAD)

Radiation source: fine focus sealed tube X-ray source

Triumph curved graphite crystal monochromator

Detector resolution: 7.4074 pixels $\mathrm{mm}^{-1}$

$\omega$ and phi scans

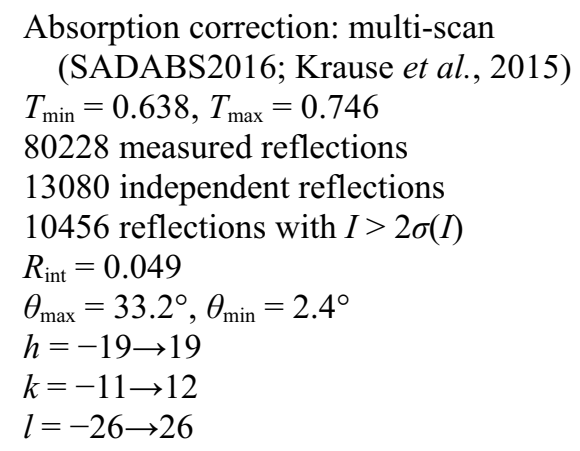

Hydrogen site location: mixed

$\mathrm{H}$ atoms treated by a mixture of independent and constrained refinement

$w=1 /\left[\sigma^{2}\left(F_{\mathrm{o}}^{2}\right)+(0.0275 P)^{2}+0.0775 P\right]$ where $P=\left(F_{\mathrm{o}}^{2}+2 F_{\mathrm{c}}{ }^{2}\right) / 3$

$(\Delta / \sigma)_{\max }<0.001$

$\Delta \rho_{\max }=0.36 \mathrm{e} \AA^{-3}$

$\Delta \rho_{\min }=-0.48$ e $\AA^{-3}$

Absolute structure: Flack $x$ determined using 4051 quotients $[(\mathrm{I}+)-(\mathrm{I}-)] /[(\mathrm{I}+)+(\mathrm{I}-)]$ (Parsons et al., 2013)

Absolute structure parameter: 0.006 (3)

Special details

Geometry. All esds (except the esd in the dihedral angle between two 1.s. planes) are estimated using the full covariance matrix. The cell esds are taken into account individually in the estimation of esds in distances, angles and torsion angles; correlations between esds in cell parameters are only used when they are defined by crystal symmetry. An approximate (isotropic) treatment of cell esds is used for estimating esds involving l.s. planes.

Fractional atomic coordinates and isotropic or equivalent isotropic displacement parameters $\left(\AA^{2}\right)$

\begin{tabular}{llllll}
\hline & $x$ & $y$ & $z$ & $U_{\text {iso }} * / U_{\text {eq }}$ & Occ. $(<1)$ \\
\hline Br1 & $1.08589(2)$ & $0.23491(3)$ & $-0.10932(2)$ & $0.03179(6)$ & \\
O1 & $0.75158(11)$ & $0.32496(17)$ & $0.21175(8)$ & $0.0226(3)$ & \\
H1B & $0.726(2)$ & $0.2891(12)$ & $0.1673(15)$ & $0.034^{*}$ & \\
O2 & $1.09216(11)$ & $0.03313(19)$ & $0.34490(8)$ & $0.0250(3)$ & \\
O3 & $0.45306(14)$ & $0.2726(2)$ & $0.05187(10)$ & $0.0416(4)$ & \\
O4 & $0.33269(13)$ & $0.4451(2)$ & $-0.01531(8)$ & $0.0313(3)$ & \\
O5 & $0.67059(14)$ & $0.2732(2)$ & $0.06194(9)$ & $0.0333(4)$ & \\
H5D & $0.678(3)$ & $0.173(5)$ & $0.0399(18)$ & $0.050^{*}$ & \\
H5E & $0.609(3)$ & $0.282(4)$ & $0.0580(18)$ & $0.050^{*}$ & \\
O6 & $1.3652(11)$ & $-0.1662(18)$ & $0.2755(8)$ & $0.061(5)$ & $0.166(7)$ \\
H6D & 1.381796 & -0.060138 & 0.281851 & $0.091 *$ & $0.166(7)$ \\
H6E & 1.295716 & -0.160377 & 0.260652 & $0.091 *$ & $0.166(7)$ \\
N1 & $0.59081(12)$ & $-0.1394(2)$ & $0.14129(9)$ & $0.0209(3)$ & \\
H1 & 0.621515 & -0.107941 & 0.094301 & $0.025^{*}$ & \\
N2 & $1.14361(13)$ & $0.0185(2)$ & $0.22591(10)$ & $0.0245(3)$ & \\
C1 & $0.89931(14)$ & $0.1917(2)$ & $0.29663(10)$ & $0.0177(3)$ &
\end{tabular}




\begin{tabular}{|c|c|c|c|c|}
\hline H1A & 0.912833 & 0.105727 & 0.338929 & $0.021^{*}$ \\
\hline $\mathrm{C} 2$ & $0.77558(14)$ & $0.1863(2)$ & $0.26328(10)$ & $0.0183(3)$ \\
\hline $\mathrm{C} 3$ & 0.74525 (14) & $0.0197(2)$ & $0.21876(10)$ & $0.0189(3)$ \\
\hline $\mathrm{H} 3 \mathrm{~A}$ & 0.773132 & 0.022355 & 0.169312 & $0.023^{*}$ \\
\hline H3B & 0.779760 & -0.075971 & 0.249780 & $0.023^{*}$ \\
\hline $\mathrm{C} 4$ & $0.62435(14)$ & $-0.0081(3)$ & $0.20190(10)$ & $0.0211(3)$ \\
\hline $\mathrm{H} 4 \mathrm{~A}$ & 0.599796 & -0.042486 & 0.250303 & $0.025^{*}$ \\
\hline $\mathrm{H} 4 \mathrm{~B}$ & 0.588573 & 0.099757 & 0.184672 & $0.025^{*}$ \\
\hline C5 & $0.62902(18)$ & $-0.3115(3)$ & $0.16528(14)$ & $0.0318(5)$ \\
\hline $\mathrm{H} 5 \mathrm{~A}$ & 0.607261 & -0.390295 & 0.122359 & $0.048^{*}$ \\
\hline H5B & 0.597537 & -0.346948 & 0.210080 & $0.048^{*}$ \\
\hline $\mathrm{H} 5 \mathrm{C}$ & 0.707487 & -0.311025 & 0.179237 & $0.048^{*}$ \\
\hline C6 & $0.47158(16)$ & $-0.1387(3)$ & $0.11978(13)$ & $0.0306(4)$ \\
\hline H6A & 0.447484 & -0.027521 & 0.099337 & $0.046^{*}$ \\
\hline H6B & 0.439568 & -0.163370 & 0.165684 & $0.046^{*}$ \\
\hline $\mathrm{H} 6 \mathrm{C}$ & 0.449243 & -0.224808 & 0.080161 & $0.046^{*}$ \\
\hline $\mathrm{C} 7$ & $0.71486(13)$ & $0.2170(3)$ & $0.33194(9)$ & $0.0202(3)$ \\
\hline $\mathrm{C} 8$ & $0.66495(17)$ & $0.3697(3)$ & $0.33479(12)$ & $0.0292(4)$ \\
\hline H8 & 0.666506 & 0.447440 & 0.293730 & $0.035^{*}$ \\
\hline C9 & $0.6113(2)$ & $0.4171(3)$ & $0.39581(13)$ & $0.0376(5)$ \\
\hline H9 & 0.578160 & 0.524760 & 0.395368 & $0.045^{*}$ \\
\hline $\mathrm{C} 10$ & $0.6071(2)$ & $0.3088(3)$ & 0.45499 (13) & $0.0365(5)$ \\
\hline H10 & 0.571006 & 0.340816 & 0.496032 & $0.044 *$ \\
\hline C11 & $0.65593(18)$ & $0.1492(3)$ & $0.45603(12)$ & $0.0315(5)$ \\
\hline $\mathrm{C} 12$ & $0.6518(2)$ & $0.0367(4)$ & $0.51821(15)$ & $0.0478(7)$ \\
\hline H12 & 0.615365 & 0.070222 & 0.558867 & $0.057^{*}$ \\
\hline C13 & $0.6981(3)$ & $-0.1164(4)$ & $0.52119(18)$ & $0.0583(8)$ \\
\hline H13 & 0.693912 & -0.189679 & 0.563465 & $0.070^{*}$ \\
\hline $\mathrm{C} 14$ & $0.7528(2)$ & $-0.1682(4)$ & $0.46177(16)$ & $0.0469(7)$ \\
\hline H14 & 0.785004 & -0.276756 & 0.463796 & $0.056^{*}$ \\
\hline C15 & $0.75993(18)$ & $-0.0622(3)$ & $0.40071(12)$ & $0.0304(4)$ \\
\hline H15 & 0.798548 & -0.098306 & 0.361670 & $0.036^{*}$ \\
\hline $\mathrm{C} 16$ & $0.71136(16)$ & $0.0992(3)$ & $0.39441(11)$ & $0.0239(4)$ \\
\hline $\mathrm{C} 17$ & 0.93134 (14) & $0.3615(2)$ & $0.33346(10)$ & $0.0187(3)$ \\
\hline $\mathrm{C} 18$ & $0.93961(18)$ & $0.3834(3)$ & $0.41341(11)$ & $0.0268(4)$ \\
\hline H18 & 0.925381 & 0.291144 & 0.444733 & $0.032 *$ \\
\hline $\mathrm{C} 19$ & $0.9683(2)$ & $0.5381(3)$ & 0.44745 (11) & $0.0327(5)$ \\
\hline H19 & 0.973412 & 0.551193 & 0.501838 & $0.039 *$ \\
\hline $\mathrm{C} 20$ & $0.98963(19)$ & $0.6738(3)$ & $0.40278(11)$ & $0.0314(5)$ \\
\hline $\mathrm{H} 20$ & 1.010481 & 0.779369 & 0.426327 & $0.038^{*}$ \\
\hline $\mathrm{C} 21$ & $0.98022(19)$ & $0.6541(3)$ & $0.32309(11)$ & $0.0292(4)$ \\
\hline $\mathrm{H} 21$ & 0.993225 & 0.747126 & 0.291782 & $0.035^{*}$ \\
\hline $\mathrm{C} 22$ & $0.95196(17)$ & $0.4994(3)$ & $0.28939(11)$ & $0.0254(4)$ \\
\hline $\mathrm{H} 22$ & 0.946527 & 0.487081 & 0.234939 & $0.031^{*}$ \\
\hline $\mathrm{C} 23$ & $0.97303(14)$ & $0.1486(2)$ & $0.23939(10)$ & 0.0188 \\
\hline $\mathrm{C} 24$ & $0.95734(14)$ & $0.1960(2)$ & $0.16317(10)$ & 0.0199 (4) \\
\hline $\mathrm{H} 24$ & 0.894360 & 0.256618 & 0.141861 & $0.024 *$ \\
\hline $\mathrm{C} 25$ & $1.03406(14)$ & 0.1555 & $0.11563(11)$ & 0.0209 \\
\hline
\end{tabular}




$\begin{array}{lllll}\text { C26 } & 1.02197(14) & 0.2073(3) & 0.03709(10) & 0.0230(4) \\ \text { H26 } & 0.961896 & 0.272839 & 0.014304 & 0.028^{*} \\ \text { C27 } & 1.09881(16) & 0.1605(3) & -0.00506(11) & 0.0239(4) \\ \text { C28 } & 1.18724(16) & 0.0604(3) & 0.02554(12) & 0.0282(4) \\ \text { H28 } & 1.237701 & 0.026726 & -0.005945 & 0.034^{*} \\ \text { C29 } & 1.19988(17) & 0.0116(3) & 0.10170(13) & 0.0289(4) \\ \text { H29 } & 1.259621 & -0.056265 & 0.123021 & 0.035^{*} \\ \text { C30 } & 1.12503(15) & 0.0612(3) & 0.14873(11) & 0.0226(4) \\ \text { C31 } & 1.07295(15) & 0.0640(2) & 0.26745(11) & 0.0207(3) \\ \text { C32 } & 1.19411(18) & -0.0419(3) & 0.37533(14) & 0.0364(5) \\ \text { H32A } & 1.197179 & -0.156057 & 0.354303 & 0.055^{*} \\ \text { H32B } & 1.202846 & -0.047604 & 0.431923 & 0.055^{*} \\ \text { H32C } & 1.251863 & 0.026661 & 0.360523 & 0.055^{*} \\ \text { C33 } & 0.37747(16) & 0.3748(3) & 0.04720(12) & 0.0258(4) \\ \text { C34 } & 0.33351(16) & 0.4182(3) & 0.11951(11) & 0.0244(4) \\ \text { C35 } & 0.23897(19) & 0.5090(3) & 0.11598(13) & 0.0331(5) \\ \text { H35 } & 0.202051 & 0.548308 & 0.067447 & 0.040^{*} \\ \text { C36 } & 0.1981(2) & 0.5426(4) & 0.18289(15) & 0.0487(7) \\ \text { H36 } & 0.133006 & 0.603990 & 0.179969 & 0.058^{*} \\ \text { C37 } & 0.2515(2) & 0.4876(5) & 0.25356(15) & 0.0531(8) \\ \text { H37 } & 0.223153 & 0.510905 & 0.299262 & 0.064^{*} \\ \text { C38 } & 0.3462(2) & 0.3986(4) & 0.25814(14) & 0.0523(8) \\ \text { H38 } & 0.383205 & 0.360736 & 0.306920 & 0.063^{*} \\ \text { C39 } & 0.3871(2) & 0.3647(4) & 0.19136(13) & 0.0394(6) \\ \text { H39 } & 0.452599 & 0.304104 & 0.194674 & 0.047^{*}\end{array}$

Atomic displacement parameters $\left(\AA^{2}\right)$

\begin{tabular}{lllllll}
\hline & $U^{11}$ & $U^{22}$ & $U^{33}$ & $U^{12}$ & $U^{13}$ & $U^{23}$ \\
\hline Br1 & $0.03261(10)$ & $0.04237(12)$ & $0.02255(8)$ & $-0.00372(11)$ & $0.01087(6)$ & $-0.00332(10)$ \\
O1 & $0.0289(7)$ & $0.0205(7)$ & $0.0173(6)$ & $0.0052(5)$ & $0.0002(5)$ & $0.0021(5)$ \\
O2 & $0.0232(6)$ & $0.0262(7)$ & $0.0241(6)$ & $0.0044(5)$ & $-0.0002(5)$ & $0.0040(6)$ \\
O3 & $0.0426(9)$ & $0.0432(12)$ & $0.0433(9)$ & $0.0160(8)$ & $0.0199(7)$ & $0.0064(7)$ \\
O4 & $0.0368(8)$ & $0.0339(8)$ & $0.0242(7)$ & $0.0011(7)$ & $0.0077(6)$ & $0.0028(6)$ \\
O5 & $0.0398(8)$ & $0.0321(10)$ & $0.0241(7)$ & $-0.0038(7)$ & $-0.0054(6)$ & $0.0037(6)$ \\
O6 & $0.062(9)$ & $0.057(9)$ & $0.070(10)$ & $0.003(6)$ & $0.029(7)$ & $-0.006(7)$ \\
N1 & $0.0207(7)$ & $0.0233(8)$ & $0.0181(7)$ & $-0.0019(6)$ & $0.0012(6)$ & $-0.0003(6)$ \\
N2 & $0.0190(7)$ & $0.0245(9)$ & $0.0302(8)$ & $0.0031(6)$ & $0.0047(6)$ & $0.0034(7)$ \\
C1 & $0.0204(7)$ & $0.0174(9)$ & $0.0154(7)$ & $0.0014(6)$ & $0.0036(6)$ & $0.0016(6)$ \\
C2 & $0.0196(7)$ & $0.0195(9)$ & $0.0159(7)$ & $0.0018(6)$ & $0.0032(6)$ & $-0.0006(6)$ \\
C3 & $0.0189(8)$ & $0.0194(9)$ & $0.0186(7)$ & $0.0017(6)$ & $0.0035(6)$ & $-0.0004(6)$ \\
C4 & $0.0192(8)$ & $0.0245(9)$ & $0.0199(8)$ & $0.0000(7)$ & $0.0044(6)$ & $-0.0035(7)$ \\
C5 & $0.0311(10)$ & $0.0230(11)$ & $0.0404(11)$ & $-0.0007(8)$ & $0.0036(9)$ & $0.0005(8)$ \\
C6 & $0.0209(9)$ & $0.0387(12)$ & $0.0301(10)$ & $-0.0047(8)$ & $-0.0022(8)$ & $0.0033(9)$ \\
C7 & $0.0202(7)$ & $0.0236(10)$ & $0.0166(6)$ & $0.0026(8)$ & $0.0023(5)$ & $-0.0022(7)$ \\
C8 & $0.0322(10)$ & $0.0302(11)$ & $0.0265(9)$ & $0.0085(9)$ & $0.0091(8)$ & $0.0004(8)$ \\
C9 & $0.0412(12)$ & $0.0380(13)$ & $0.0367(12)$ & $0.0113(10)$ & $0.0158(10)$ & $-0.0068(10)$ \\
C10 & $0.0343(11)$ & $0.0499(14)$ & $0.0285(10)$ & $0.0041(10)$ & $0.0144(9)$ & $-0.0078(10)$
\end{tabular}




\begin{tabular}{|c|c|c|c|c|c|c|}
\hline $\mathrm{C} 11$ & $0.0292(11)$ & $0.0426(13)$ & $0.0251(9)$ & $-0.0040(10)$ & $0.0112(8)$ & $-0.0013(9)$ \\
\hline $\mathrm{C} 12$ & $0.0601(17)$ & $0.0552(17)$ & $0.0344(12)$ & $0.0006(14)$ & $0.0257(12)$ & $0.0081(12)$ \\
\hline $\mathrm{C} 13$ & $0.080(2)$ & 0.0565 (19) & $0.0468(16)$ & $0.0065(16)$ & $0.0342(15)$ & $0.0234(14)$ \\
\hline C14 & $0.0606(17)$ & $0.0397(15)$ & $0.0450(14)$ & $0.0093(12)$ & $0.0225(12)$ & $0.0186(12)$ \\
\hline $\mathrm{C} 15$ & $0.0355(11)$ & $0.0291(11)$ & $0.0284(10)$ & $0.0000(9)$ & $0.0105(8)$ & $0.0047(8)$ \\
\hline $\mathrm{C} 16$ & $0.0234(9)$ & $0.0281(10)$ & $0.0205(8)$ & $-0.0027(7)$ & $0.0050(7)$ & $-0.0008(7)$ \\
\hline $\mathrm{C} 17$ & $0.0206(8)$ & $0.0189(8)$ & $0.0159(7)$ & $0.0014(7)$ & $0.0008(6)$ & $0.0010(6)$ \\
\hline $\mathrm{C} 18$ & $0.0401(11)$ & $0.0225(10)$ & $0.0165(8)$ & $-0.0047(8)$ & 0.0008 (7) & $0.0027(7)$ \\
\hline C19 & $0.0522(13)$ & $0.0290(11)$ & $0.0154(8)$ & $-0.0069(10)$ & $0.0018(8)$ & $-0.0016(8)$ \\
\hline $\mathrm{C} 20$ & $0.0500(13)$ & $0.0219(10)$ & $0.0209(9)$ & $-0.0066(9)$ & $0.0020(8)$ & $-0.0027(8)$ \\
\hline $\mathrm{C} 21$ & $0.0471(12)$ & $0.0200(10)$ & $0.0206(8)$ & $-0.0058(9)$ & $0.0058(8)$ & $0.0013(7)$ \\
\hline $\mathrm{C} 22$ & $0.0379(11)$ & $0.0223(10)$ & $0.0169(8)$ & $-0.0046(8)$ & $0.0068(7)$ & $0.0003(7)$ \\
\hline $\mathrm{C} 23$ & $0.0179(8)$ & $0.0164(9)$ & $0.0220(8)$ & $0.0001(6)$ & $0.0032(6)$ & $-0.0011(7)$ \\
\hline $\mathrm{C} 24$ & $0.0184(7)$ & $0.0212(10)$ & $0.0204(7)$ & $0.0026(6)$ & $0.0042(6)$ & $0.0005(6)$ \\
\hline $\mathrm{C} 25$ & $0.0188(8)$ & $0.0212(9)$ & $0.0238(8)$ & $-0.0004(7)$ & $0.0063(6)$ & $-0.0015(7)$ \\
\hline C26 & $0.0221(8)$ & $0.0252(11)$ & $0.0223(7)$ & $0.0006(7)$ & $0.0054(6)$ & $-0.0013(7)$ \\
\hline $\mathrm{C} 27$ & $0.0259(9)$ & $0.0238(9)$ & $0.0237(8)$ & $-0.0052(7)$ & $0.0093(7)$ & $-0.0043(8)$ \\
\hline $\mathrm{C} 28$ & $0.0222(9)$ & $0.0302(11)$ & $0.0349(11)$ & $-0.0013(8)$ & $0.0125(8)$ & $-0.0059(9)$ \\
\hline $\mathrm{C} 29$ & $0.0212(9)$ & $0.0293(11)$ & $0.0378(11)$ & $0.0035(8)$ & $0.0100(8)$ & $0.0003(9)$ \\
\hline $\mathrm{C} 30$ & $0.0195(8)$ & $0.0209(9)$ & $0.0283(9)$ & $0.0004(7)$ & $0.0062(7)$ & $0.0001(7)$ \\
\hline $\mathrm{C} 31$ & $0.0200(8)$ & $0.0174(8)$ & $0.0238(8)$ & $-0.0014(7)$ & $0.0013(7)$ & $0.0011(7)$ \\
\hline $\mathrm{C} 32$ & $0.0299(11)$ & 0.0417 (14) & $0.0333(11)$ & $0.0097(10)$ & $-0.0070(9)$ & $0.0046(10)$ \\
\hline $\mathrm{C} 33$ & $0.0273(9)$ & $0.0243(10)$ & $0.0272(9)$ & $-0.0023(8)$ & $0.0083(7)$ & $0.0008(8)$ \\
\hline $\mathrm{C} 34$ & $0.0254(9)$ & $0.0241(10)$ & $0.0240(9)$ & $0.0001(7)$ & $0.0049(7)$ & $-0.0013(7)$ \\
\hline $\mathrm{C} 35$ & $0.0313(11)$ & 0.0417 (14) & $0.0259(10)$ & $0.0102(10)$ & $0.0042(8)$ & $0.0009(9)$ \\
\hline $\mathrm{C} 36$ & 0.0409 (13) & $0.0683(19)$ & $0.0383(13)$ & $0.0234(13)$ & $0.0110(11)$ & $-0.0028(13)$ \\
\hline $\mathrm{C} 37$ & $0.0581(17)$ & $0.076(2)$ & $0.0279(11)$ & $0.0201(15)$ & $0.0141(11)$ & $-0.0082(13)$ \\
\hline C38 & $0.0554(16)$ & $0.076(2)$ & $0.0248(11)$ & $0.0252(15)$ & $0.0053(11)$ & $0.0020(12)$ \\
\hline C39 & $0.0383(12)$ & $0.0507(15)$ & $0.0294(11)$ & $0.0190(11)$ & $0.0061(9)$ & $0.0023(10)$ \\
\hline
\end{tabular}

Geometric parameters $\left(\AA,{ }^{o}\right)$

\begin{tabular}{llll}
\hline $\mathrm{Br} 1-\mathrm{C} 27$ & $1.900(2)$ & $\mathrm{C} 13-\mathrm{C} 14$ & $1.406(4)$ \\
$\mathrm{O} 1-\mathrm{C} 2$ & $1.422(2)$ & $\mathrm{C} 13-\mathrm{H} 13$ & 0.9500 \\
$\mathrm{O} 1-\mathrm{H} 1 \mathrm{~B}$ & $0.84(3)$ & $\mathrm{C} 14-\mathrm{C} 15$ & $1.375(3)$ \\
$\mathrm{O} 2-\mathrm{C} 31$ & $1.360(2)$ & $\mathrm{C} 14-\mathrm{H} 14$ & 0.9500 \\
$\mathrm{O} 2-\mathrm{C} 32$ & $1.437(2)$ & $\mathrm{C} 15-\mathrm{C} 16$ & $1.414(3)$ \\
$\mathrm{O} 3-\mathrm{C} 33$ & $1.244(3)$ & $\mathrm{C} 15-\mathrm{H} 15$ & 0.9500 \\
$\mathrm{O} 4-\mathrm{C} 33$ & $1.273(3)$ & $\mathrm{C} 17-\mathrm{C} 22$ & $1.388(3)$ \\
$\mathrm{O} 5-\mathrm{H} 5 \mathrm{D}$ & $0.90(4)$ & $\mathrm{C} 17-\mathrm{C} 18$ & $1.398(3)$ \\
$\mathrm{O} 5-\mathrm{H} 5 \mathrm{E}$ & $0.77(3)$ & $\mathrm{C} 18-\mathrm{C} 19$ & $1.385(3)$ \\
$\mathrm{O} 6-\mathrm{H} 6 \mathrm{D}$ & 0.8692 & $\mathrm{C} 18-\mathrm{H} 18$ & 0.9500 \\
$\mathrm{O} 6-\mathrm{H} 6 \mathrm{E}$ & 0.8735 & $\mathrm{C} 19-\mathrm{C} 20$ & $1.383(3)$ \\
$\mathrm{N} 1-\mathrm{C} 5$ & $1.484(3)$ & $\mathrm{C} 19-\mathrm{H} 19$ & 0.9500 \\
$\mathrm{~N} 1-\mathrm{C} 6$ & $1.489(2)$ & $\mathrm{C} 20-\mathrm{C} 21$ & $1.390(3)$ \\
$\mathrm{N} 1-\mathrm{C} 4$ & $1.495(2)$ & $\mathrm{C} 20-\mathrm{H} 20$ & 0.9500 \\
$\mathrm{~N} 1-\mathrm{H} 1$ & 1.0000 & $\mathrm{C} 21-\mathrm{C} 22$ & $1.381(3)$ \\
$\mathrm{N} 2-\mathrm{C} 31$ & $1.296(2)$ & $\mathrm{C} 21-\mathrm{H} 21$ & 0.9500
\end{tabular}




\begin{tabular}{|c|c|c|c|}
\hline $\mathrm{N} 2-\mathrm{C} 30$ & $1.375(3)$ & $\mathrm{C} 22-\mathrm{H} 22$ & 0.9500 \\
\hline $\mathrm{C} 1-\mathrm{C} 17$ & $1.517(2)$ & $\mathrm{C} 23-\mathrm{C} 24$ & $1.369(2)$ \\
\hline $\mathrm{C} 1-\mathrm{C} 23$ & $1.520(2)$ & $\mathrm{C} 23-\mathrm{C} 31$ & $1.440(3)$ \\
\hline $\mathrm{C} 1-\mathrm{C} 2$ & $1.574(2)$ & $\mathrm{C} 24-\mathrm{C} 25$ & $1.419(2)$ \\
\hline $\mathrm{C} 1-\mathrm{H} 1 \mathrm{~A}$ & 1.0000 & $\mathrm{C} 24-\mathrm{H} 24$ & 0.9500 \\
\hline $\mathrm{C} 2-\mathrm{C} 3$ & $1.548(3)$ & $\mathrm{C} 25-\mathrm{C} 30$ & $1.410(3)$ \\
\hline $\mathrm{C} 2-\mathrm{C} 7$ & $1.553(2)$ & $\mathrm{C} 25-\mathrm{C} 26$ & $1.419(3)$ \\
\hline $\mathrm{C} 3-\mathrm{C} 4$ & $1.522(2)$ & $\mathrm{C} 26-\mathrm{C} 27$ & $1.368(3)$ \\
\hline $\mathrm{C} 3-\mathrm{H} 3 \mathrm{~A}$ & 0.9900 & $\mathrm{C} 26-\mathrm{H} 26$ & 0.9500 \\
\hline C $3-\mathrm{H} 3 \mathrm{~B}$ & 0.9900 & $\mathrm{C} 27-\mathrm{C} 28$ & $1.401(3)$ \\
\hline $\mathrm{C} 4-\mathrm{H} 4 \mathrm{~A}$ & 0.9900 & $\mathrm{C} 28-\mathrm{C} 29$ & $1.372(3)$ \\
\hline $\mathrm{C} 4-\mathrm{H} 4 \mathrm{~B}$ & 0.9900 & $\mathrm{C} 28-\mathrm{H} 28$ & 0.9500 \\
\hline $\mathrm{C} 5-\mathrm{H} 5 \mathrm{~A}$ & 0.9800 & $\mathrm{C} 29-\mathrm{C} 30$ & $1.413(3)$ \\
\hline C5-H5B & 0.9800 & $\mathrm{C} 29-\mathrm{H} 29$ & 0.9500 \\
\hline $\mathrm{C} 5-\mathrm{H} 5 \mathrm{C}$ & 0.9800 & $\mathrm{C} 32-\mathrm{H} 32 \mathrm{~A}$ & 0.9800 \\
\hline C6-H6A & 0.9800 & $\mathrm{C} 32-\mathrm{H} 32 \mathrm{~B}$ & 0.9800 \\
\hline C6-H6B & 0.9800 & $\mathrm{C} 32-\mathrm{H} 32 \mathrm{C}$ & 0.9800 \\
\hline C6- $\mathrm{H} 6 \mathrm{C}$ & 0.9800 & $\mathrm{C} 33-\mathrm{C} 34$ & $1.508(3)$ \\
\hline $\mathrm{C} 7-\mathrm{C} 8$ & $1.369(3)$ & $\mathrm{C} 34-\mathrm{C} 35$ & $1.387(3)$ \\
\hline $\mathrm{C} 7-\mathrm{C} 16$ & $1.446(3)$ & C34-C39 & $1.390(3)$ \\
\hline $\mathrm{C} 8-\mathrm{C} 9$ & $1.412(3)$ & $\mathrm{C} 35-\mathrm{C} 36$ & $1.385(3)$ \\
\hline $\mathrm{C} 8-\mathrm{H} 8$ & 0.9500 & C35-H35 & 0.9500 \\
\hline $\mathrm{C} 9-\mathrm{C} 10$ & $1.354(4)$ & $\mathrm{C} 36-\mathrm{C} 37$ & $1.376(4)$ \\
\hline C9- $\mathrm{H} 9$ & 0.9500 & C36- & 0.9500 \\
\hline $\mathrm{C} 10-\mathrm{C} 11$ & $1.406(3)$ & C $37-\mathrm{C} 38$ & $1.380(4)$ \\
\hline $\mathrm{C} 10-\mathrm{H} 10$ & 0.9500 & C37-H37 & 0.9500 \\
\hline $\mathrm{C} 11-\mathrm{C} 12$ & $1.416(3)$ & C $38-\mathrm{C} 39$ & $1.384(3)$ \\
\hline $\mathrm{C} 11-\mathrm{C} 16$ & $1.440(3)$ & C $38-\mathrm{H} 38$ & 0.9500 \\
\hline $\mathrm{C} 12-\mathrm{C} 13$ & $1.345(5)$ & С $39-\mathrm{H} 39$ & 0.9500 \\
\hline $\mathrm{C} 12-\mathrm{H} 12$ & 0.9500 & & \\
\hline $\mathrm{C} 2-\mathrm{O} 1-\mathrm{H} 1 \mathrm{~B}$ & 109.5 & $\mathrm{C} 15-\mathrm{C} 16-\mathrm{C} 7$ & $125.05(18)$ \\
\hline $\mathrm{C} 31-\mathrm{O} 2-\mathrm{C} 32$ & $116.16(16)$ & $\mathrm{C} 11-\mathrm{C} 16-\mathrm{C} 7$ & $118.14(19)$ \\
\hline $\mathrm{H} 5 \mathrm{D}-\mathrm{O} 5-\mathrm{H} 5 \mathrm{E}$ & $103(3)$ & $\mathrm{C} 22-\mathrm{C} 17-\mathrm{C} 18$ & $118.08(17)$ \\
\hline $\mathrm{H} 6 \mathrm{D}-\mathrm{O} 6-\mathrm{H} 6 \mathrm{E}$ & 101.2 & $\mathrm{C} 22-\mathrm{C} 17-\mathrm{C} 1$ & $121.56(15)$ \\
\hline $\mathrm{C} 5-\mathrm{N} 1-\mathrm{C} 6$ & $109.94(17)$ & $\mathrm{C} 18-\mathrm{C} 17-\mathrm{C} 1$ & $120.36(16)$ \\
\hline $\mathrm{C} 5-\mathrm{N} 1-\mathrm{C} 4$ & $113.54(15)$ & $\mathrm{C} 19-\mathrm{C} 18-\mathrm{C} 17$ & $120.74(18)$ \\
\hline $\mathrm{C} 6-\mathrm{N} 1-\mathrm{C} 4$ & $109.21(16)$ & $\mathrm{C} 19-\mathrm{C} 18-\mathrm{H} 18$ & 119.6 \\
\hline $\mathrm{C} 5-\mathrm{N} 1-\mathrm{H} 1$ & 108.0 & $\mathrm{C} 17-\mathrm{C} 18-\mathrm{H} 18$ & 119.6 \\
\hline $\mathrm{C} 6-\mathrm{N} 1-\mathrm{H} 1$ & 108.0 & $\mathrm{C} 20-\mathrm{C} 19-\mathrm{C} 18$ & $120.44(18)$ \\
\hline $\mathrm{C} 4-\mathrm{N} 1-\mathrm{H} 1$ & 108.0 & $\mathrm{C} 20-\mathrm{C} 19-\mathrm{H} 19$ & 119.8 \\
\hline $\mathrm{C} 31-\mathrm{N} 2-\mathrm{C} 30$ & $117.76(16)$ & $\mathrm{C} 18-\mathrm{C} 19-\mathrm{H} 19$ & 119.8 \\
\hline $\mathrm{C} 17-\mathrm{C} 1-\mathrm{C} 23$ & $109.33(14)$ & $\mathrm{C} 19-\mathrm{C} 20-\mathrm{C} 21$ & $119.3(2)$ \\
\hline $\mathrm{C} 17-\mathrm{C} 1-\mathrm{C} 2$ & $110.99(14)$ & $\mathrm{C} 19-\mathrm{C} 20-\mathrm{H} 20$ & 120.3 \\
\hline $\mathrm{C} 23-\mathrm{C} 1-\mathrm{C} 2$ & $115.54(14)$ & $\mathrm{C} 21-\mathrm{C} 20-\mathrm{H} 20$ & 120.3 \\
\hline $\mathrm{C} 17-\mathrm{C} 1-\mathrm{H} 1 \mathrm{~A}$ & 106.8 & $\mathrm{C} 22-\mathrm{C} 21-\mathrm{C} 20$ & $120.05(19)$ \\
\hline $\mathrm{C} 23-\mathrm{C} 1-\mathrm{H} 1 \mathrm{~A}$ & 106.8 & $\mathrm{C} 22-\mathrm{C} 21-\mathrm{H} 21$ & 120.0 \\
\hline $\mathrm{C} 2-\mathrm{C} 1-\mathrm{H} 1 \mathrm{~A}$ & 106.8 & $\mathrm{C} 20-\mathrm{C} 21-\mathrm{H} 21$ & 120.0 \\
\hline
\end{tabular}




\begin{tabular}{|c|c|c|c|}
\hline $\mathrm{O} 1-\mathrm{C} 2-\mathrm{C} 3$ & $109.36(14)$ & $\mathrm{C} 21-\mathrm{C} 22-\mathrm{C} 17$ & $121.36(17)$ \\
\hline $\mathrm{O} 1-\mathrm{C} 2-\mathrm{C} 7$ & $106.90(15)$ & $\mathrm{C} 21-\mathrm{C} 22-\mathrm{H} 22$ & 119.3 \\
\hline $\mathrm{C} 3-\mathrm{C} 2-\mathrm{C} 7$ & $114.32(15)$ & $\mathrm{C} 17-\mathrm{C} 22-\mathrm{H} 22$ & 119.3 \\
\hline $\mathrm{O} 1-\mathrm{C} 2-\mathrm{C} 1$ & $107.77(14)$ & $\mathrm{C} 24-\mathrm{C} 23-\mathrm{C} 31$ & $115.67(16)$ \\
\hline $\mathrm{C} 3-\mathrm{C} 2-\mathrm{C} 1$ & $110.81(14)$ & $\mathrm{C} 24-\mathrm{C} 23-\mathrm{C} 1$ & $125.41(16)$ \\
\hline $\mathrm{C} 7-\mathrm{C} 2-\mathrm{C} 1$ & $107.42(13)$ & $\mathrm{C} 31-\mathrm{C} 23-\mathrm{C} 1$ & $118.68(16)$ \\
\hline $\mathrm{C} 4-\mathrm{C} 3-\mathrm{C} 2$ & $112.06(15)$ & $\mathrm{C} 23-\mathrm{C} 24-\mathrm{C} 25$ & $120.77(16)$ \\
\hline $\mathrm{C} 4-\mathrm{C} 3-\mathrm{H} 3 \mathrm{~A}$ & 109.2 & $\mathrm{C} 23-\mathrm{C} 24-\mathrm{H} 24$ & 119.6 \\
\hline $\mathrm{C} 2-\mathrm{C} 3-\mathrm{H} 3 \mathrm{~A}$ & 109.2 & $\mathrm{C} 25-\mathrm{C} 24-\mathrm{H} 24$ & 119.6 \\
\hline $\mathrm{C} 4-\mathrm{C} 3-\mathrm{H} 3 \mathrm{~B}$ & 109.2 & $\mathrm{C} 30-\mathrm{C} 25-\mathrm{C} 24$ & $117.98(17)$ \\
\hline $\mathrm{C} 2-\mathrm{C} 3-\mathrm{H} 3 \mathrm{~B}$ & 109.2 & $\mathrm{C} 30-\mathrm{C} 25-\mathrm{C} 26$ & $119.80(16)$ \\
\hline $\mathrm{H} 3 \mathrm{~A}-\mathrm{C} 3-\mathrm{H} 3 \mathrm{~B}$ & 107.9 & $\mathrm{C} 24-\mathrm{C} 25-\mathrm{C} 26$ & $122.23(17)$ \\
\hline $\mathrm{N} 1-\mathrm{C} 4-\mathrm{C} 3$ & $113.09(15)$ & $\mathrm{C} 27-\mathrm{C} 26-\mathrm{C} 25$ & $118.48(18)$ \\
\hline $\mathrm{N} 1-\mathrm{C} 4-\mathrm{H} 4 \mathrm{~A}$ & 109.0 & $\mathrm{C} 27-\mathrm{C} 26-\mathrm{H} 26$ & 120.8 \\
\hline $\mathrm{C} 3-\mathrm{C} 4-\mathrm{H} 4 \mathrm{~A}$ & 109.0 & $\mathrm{C} 25-\mathrm{C} 26-\mathrm{H} 26$ & 120.8 \\
\hline $\mathrm{N} 1-\mathrm{C} 4-\mathrm{H} 4 \mathrm{~B}$ & 109.0 & $\mathrm{C} 26-\mathrm{C} 27-\mathrm{C} 28$ & $122.72(19)$ \\
\hline $\mathrm{C} 3-\mathrm{C} 4-\mathrm{H} 4 \mathrm{~B}$ & 109.0 & $\mathrm{C} 26-\mathrm{C} 27-\mathrm{Br} 1$ & $118.87(16)$ \\
\hline $\mathrm{H} 4 \mathrm{~A}-\mathrm{C} 4-\mathrm{H} 4 \mathrm{~B}$ & 107.8 & $\mathrm{C} 28-\mathrm{C} 27-\mathrm{Br} 1$ & $118.41(14)$ \\
\hline $\mathrm{N} 1-\mathrm{C} 5-\mathrm{H} 5 \mathrm{~A}$ & 109.5 & $\mathrm{C} 29-\mathrm{C} 28-\mathrm{C} 27$ & $118.98(18)$ \\
\hline $\mathrm{N} 1-\mathrm{C} 5-\mathrm{H} 5 \mathrm{~B}$ & 109.5 & $\mathrm{C} 29-\mathrm{C} 28-\mathrm{H} 28$ & 120.5 \\
\hline $\mathrm{H} 5 \mathrm{~A}-\mathrm{C} 5-\mathrm{H} 5 \mathrm{~B}$ & 109.5 & $\mathrm{C} 27-\mathrm{C} 28-\mathrm{H} 28$ & 120.5 \\
\hline $\mathrm{N} 1-\mathrm{C} 5-\mathrm{H} 5 \mathrm{C}$ & 109.5 & $\mathrm{C} 28-\mathrm{C} 29-\mathrm{C} 30$ & $120.7(2)$ \\
\hline $\mathrm{H} 5 \mathrm{~A}-\mathrm{C} 5-\mathrm{H} 5 \mathrm{C}$ & 109.5 & $\mathrm{C} 28-\mathrm{C} 29-\mathrm{H} 29$ & 119.6 \\
\hline $\mathrm{H} 5 \mathrm{~B}-\mathrm{C} 5-\mathrm{H} 5 \mathrm{C}$ & 109.5 & $\mathrm{C} 30-\mathrm{C} 29-\mathrm{H} 29$ & 119.6 \\
\hline $\mathrm{N} 1-\mathrm{C} 6-\mathrm{H} 6 \mathrm{~A}$ & 109.5 & $\mathrm{~N} 2-\mathrm{C} 30-\mathrm{C} 25$ & $121.68(17)$ \\
\hline $\mathrm{N} 1-\mathrm{C} 6-\mathrm{H} 6 \mathrm{~B}$ & 109.5 & $\mathrm{~N} 2-\mathrm{C} 30-\mathrm{C} 29$ & $119.12(18)$ \\
\hline $\mathrm{H} 6 \mathrm{~A}-\mathrm{C} 6-\mathrm{H} 6 \mathrm{~B}$ & 109.5 & $\mathrm{C} 25-\mathrm{C} 30-\mathrm{C} 29$ & $119.19(18)$ \\
\hline $\mathrm{N} 1-\mathrm{C} 6-\mathrm{H} 6 \mathrm{C}$ & 109.5 & $\mathrm{~N} 2-\mathrm{C} 31-\mathrm{O} 2$ & $119.21(17)$ \\
\hline $\mathrm{H} 6 \mathrm{~A}-\mathrm{C} 6-\mathrm{H} 6 \mathrm{C}$ & 109.5 & $\mathrm{~N} 2-\mathrm{C} 31-\mathrm{C} 23$ & $125.87(17)$ \\
\hline $\mathrm{H} 6 \mathrm{~B}-\mathrm{C} 6-\mathrm{H} 6 \mathrm{C}$ & 109.5 & $\mathrm{O} 2-\mathrm{C} 31-\mathrm{C} 23$ & $114.92(16)$ \\
\hline $\mathrm{C} 8-\mathrm{C} 7-\mathrm{C} 16$ & $118.01(16)$ & $\mathrm{O} 2-\mathrm{C} 32-\mathrm{H} 32 \mathrm{~A}$ & 109.5 \\
\hline $\mathrm{C} 8-\mathrm{C} 7-\mathrm{C} 2$ & $117.30(17)$ & $\mathrm{O} 2-\mathrm{C} 32-\mathrm{H} 32 \mathrm{~B}$ & 109.5 \\
\hline $\mathrm{C} 16-\mathrm{C} 7-\mathrm{C} 2$ & $124.64(18)$ & $\mathrm{H} 32 \mathrm{~A}-\mathrm{C} 32-\mathrm{H} 32 \mathrm{~B}$ & 109.5 \\
\hline $\mathrm{C} 7-\mathrm{C} 8-\mathrm{C} 9$ & $123.1(2)$ & $\mathrm{O} 2-\mathrm{C} 32-\mathrm{H} 32 \mathrm{C}$ & 109.5 \\
\hline $\mathrm{C} 7-\mathrm{C} 8-\mathrm{H} 8$ & 118.4 & $\mathrm{H} 32 \mathrm{~A}-\mathrm{C} 32-\mathrm{H} 32 \mathrm{C}$ & 109.5 \\
\hline $\mathrm{C} 9-\mathrm{C} 8-\mathrm{H} 8$ & 118.4 & $\mathrm{H} 32 \mathrm{~B}-\mathrm{C} 32-\mathrm{H} 32 \mathrm{C}$ & 109.5 \\
\hline $\mathrm{C} 10-\mathrm{C} 9-\mathrm{C} 8$ & $119.9(2)$ & $\mathrm{O} 3-\mathrm{C} 33-\mathrm{O} 4$ & $124.42(19)$ \\
\hline $\mathrm{C} 10-\mathrm{C} 9-\mathrm{H} 9$ & 120.1 & $\mathrm{O} 3-\mathrm{C} 33-\mathrm{C} 34$ & $118.83(19)$ \\
\hline $\mathrm{C} 8-\mathrm{C} 9-\mathrm{H} 9$ & 120.1 & $\mathrm{O} 4-\mathrm{C} 33-\mathrm{C} 34$ & $116.74(18)$ \\
\hline $\mathrm{C} 9-\mathrm{C} 10-\mathrm{C} 11$ & $120.4(2)$ & $\mathrm{C} 35-\mathrm{C} 34-\mathrm{C} 39$ & $118.76(19)$ \\
\hline $\mathrm{C} 9-\mathrm{C} 10-\mathrm{H} 10$ & 119.8 & $\mathrm{C} 35-\mathrm{C} 34-\mathrm{C} 33$ & $121.28(18)$ \\
\hline $\mathrm{C} 11-\mathrm{C} 10-\mathrm{H} 10$ & 119.8 & $\mathrm{C} 39-\mathrm{C} 34-\mathrm{C} 33$ & 119.95 (19) \\
\hline $\mathrm{C} 10-\mathrm{C} 11-\mathrm{C} 12$ & $120.2(2)$ & $\mathrm{C} 36-\mathrm{C} 35-\mathrm{C} 34$ & $120.3(2)$ \\
\hline $\mathrm{C} 10-\mathrm{C} 11-\mathrm{C} 16$ & $120.5(2)$ & $\mathrm{C} 36-\mathrm{C} 35-\mathrm{H} 35$ & 119.8 \\
\hline $\mathrm{C} 12-\mathrm{C} 11-\mathrm{C} 16$ & $119.3(2)$ & $\mathrm{C} 34-\mathrm{C} 35-\mathrm{H} 35$ & 119.8 \\
\hline $\mathrm{C} 13-\mathrm{C} 12-\mathrm{C} 11$ & $121.7(2)$ & $\mathrm{C} 37-\mathrm{C} 36-\mathrm{C} 35$ & $120.3(2)$ \\
\hline $\mathrm{C} 13-\mathrm{C} 12-\mathrm{H} 12$ & 119.2 & $\mathrm{C} 37-\mathrm{C} 36-\mathrm{H} 36$ & 119.9 \\
\hline $\mathrm{C} 11-\mathrm{C} 12-\mathrm{H} 12$ & 119.2 & $\mathrm{C} 35-\mathrm{C} 36-\mathrm{H} 36$ & 119.9 \\
\hline
\end{tabular}




\begin{tabular}{|c|c|c|c|}
\hline $\mathrm{C} 12-\mathrm{C} 13-\mathrm{C} 14$ & $120.0(2)$ & $\mathrm{C} 36-\mathrm{C} 37-\mathrm{C} 38$ & $120.1(2)$ \\
\hline $\mathrm{C} 12-\mathrm{C} 13-\mathrm{H} 13$ & 120.0 & $\mathrm{C} 36-\mathrm{C} 37-\mathrm{H} 37$ & 120.0 \\
\hline $\mathrm{C} 14-\mathrm{C} 13-\mathrm{H} 13$ & 120.0 & $\mathrm{C} 38-\mathrm{C} 37-\mathrm{H} 37$ & 120.0 \\
\hline $\mathrm{C} 15-\mathrm{C} 14-\mathrm{C} 13$ & $120.3(3)$ & $\mathrm{C} 37-\mathrm{C} 38-\mathrm{C} 39$ & $119.7(2)$ \\
\hline $\mathrm{C} 15-\mathrm{C} 14-\mathrm{H} 14$ & 119.9 & $\mathrm{C} 37-\mathrm{C} 38-\mathrm{H} 38$ & 120.1 \\
\hline $\mathrm{C} 13-\mathrm{C} 14-\mathrm{H} 14$ & 119.9 & $\mathrm{C} 39-\mathrm{C} 38-\mathrm{H} 38$ & 120.1 \\
\hline $\mathrm{C} 14-\mathrm{C} 15-\mathrm{C} 16$ & $121.9(2)$ & $\mathrm{C} 38-\mathrm{C} 39-\mathrm{C} 34$ & $120.8(2)$ \\
\hline $\mathrm{C} 14-\mathrm{C} 15-\mathrm{H} 15$ & 119.1 & $\mathrm{C} 38-\mathrm{C} 39-\mathrm{H} 39$ & 119.6 \\
\hline $\mathrm{C} 16-\mathrm{C} 15-\mathrm{H} 15$ & 119.1 & $\mathrm{C} 34-\mathrm{C} 39-\mathrm{H} 39$ & 119.6 \\
\hline $\mathrm{C} 15-\mathrm{C} 16-\mathrm{C} 11$ & $116.81(19)$ & & \\
\hline $\mathrm{C} 17-\mathrm{C} 1-\mathrm{C} 2-\mathrm{O} 1$ & $54.82(17)$ & $\mathrm{C} 19-\mathrm{C} 20-\mathrm{C} 21-\mathrm{C} 22$ & $-1.3(4)$ \\
\hline $\mathrm{C} 23-\mathrm{C} 1-\mathrm{C} 2-\mathrm{O} 1$ & $-70.37(18)$ & $\mathrm{C} 20-\mathrm{C} 21-\mathrm{C} 22-\mathrm{C} 17$ & $0.7(3)$ \\
\hline $\mathrm{C} 17-\mathrm{C} 1-\mathrm{C} 2-\mathrm{C} 3$ & $174.44(14)$ & $\mathrm{C} 18-\mathrm{C} 17-\mathrm{C} 22-\mathrm{C} 21$ & $0.2(3)$ \\
\hline $\mathrm{C} 23-\mathrm{C} 1-\mathrm{C} 2-\mathrm{C} 3$ & $49.3(2)$ & $\mathrm{C} 1-\mathrm{C} 17-\mathrm{C} 22-\mathrm{C} 21$ & $179.44(19)$ \\
\hline $\mathrm{C} 17-\mathrm{C} 1-\mathrm{C} 2-\mathrm{C} 7$ & $-60.05(19)$ & $\mathrm{C} 17-\mathrm{C} 1-\mathrm{C} 23-\mathrm{C} 24$ & $-87.3(2)$ \\
\hline $\mathrm{C} 23-\mathrm{C} 1-\mathrm{C} 2-\mathrm{C} 7$ & $174.77(16)$ & $\mathrm{C} 2-\mathrm{C} 1-\mathrm{C} 23-\mathrm{C} 24$ & $38.7(2)$ \\
\hline $\mathrm{O} 1-\mathrm{C} 2-\mathrm{C} 3-\mathrm{C} 4$ & $-74.49(18)$ & $\mathrm{C} 17-\mathrm{C} 1-\mathrm{C} 23-\mathrm{C} 31$ & $86.83(19)$ \\
\hline $\mathrm{C} 7-\mathrm{C} 2-\mathrm{C} 3-\mathrm{C} 4$ & $45.3(2)$ & $\mathrm{C} 2-\mathrm{C} 1-\mathrm{C} 23-\mathrm{C} 31$ & $-147.14(16)$ \\
\hline $\mathrm{C} 1-\mathrm{C} 2-\mathrm{C} 3-\mathrm{C} 4$ & $166.84(14)$ & $\mathrm{C} 31-\mathrm{C} 23-\mathrm{C} 24-\mathrm{C} 25$ & $2.7(3)$ \\
\hline $\mathrm{C} 5-\mathrm{N} 1-\mathrm{C} 4-\mathrm{C} 3$ & $65.8(2)$ & $\mathrm{C} 1-\mathrm{C} 23-\mathrm{C} 24-\mathrm{C} 25$ & $177.00(17)$ \\
\hline $\mathrm{C} 6-\mathrm{N} 1-\mathrm{C} 4-\mathrm{C} 3$ & $-171.11(16)$ & $\mathrm{C} 23-\mathrm{C} 24-\mathrm{C} 25-\mathrm{C} 30$ & $1.8(3)$ \\
\hline $\mathrm{C} 2-\mathrm{C} 3-\mathrm{C} 4-\mathrm{N} 1$ & $164.04(15)$ & $\mathrm{C} 23-\mathrm{C} 24-\mathrm{C} 25-\mathrm{C} 26$ & $-177.98(18)$ \\
\hline $\mathrm{O} 1-\mathrm{C} 2-\mathrm{C} 7-\mathrm{C} 8$ & $-7.6(2)$ & $\mathrm{C} 30-\mathrm{C} 25-\mathrm{C} 26-\mathrm{C} 27$ & $1.3(3)$ \\
\hline $\mathrm{C} 3-\mathrm{C} 2-\mathrm{C} 7-\mathrm{C} 8$ & $-128.77(19)$ & $\mathrm{C} 24-\mathrm{C} 25-\mathrm{C} 26-\mathrm{C} 27$ & $-178.92(18)$ \\
\hline $\mathrm{C} 1-\mathrm{C} 2-\mathrm{C} 7-\mathrm{C} 8$ & $107.84(18)$ & $\mathrm{C} 25-\mathrm{C} 26-\mathrm{C} 27-\mathrm{C} 28$ & $1.7(3)$ \\
\hline $\mathrm{O} 1-\mathrm{C} 2-\mathrm{C} 7-\mathrm{C} 16$ & $175.18(17)$ & $\mathrm{C} 25-\mathrm{C} 26-\mathrm{C} 27-\mathrm{Br} 1$ & $-178.04(14)$ \\
\hline $\mathrm{C} 3-\mathrm{C} 2-\mathrm{C} 7-\mathrm{C} 16$ & $54.0(2)$ & $\mathrm{C} 26-\mathrm{C} 27-\mathrm{C} 28-\mathrm{C} 29$ & $-2.4(3)$ \\
\hline $\mathrm{C} 1-\mathrm{C} 2-\mathrm{C} 7-\mathrm{C} 16$ & $-69.4(2)$ & $\mathrm{Br} 1-\mathrm{C} 27-\mathrm{C} 28-\mathrm{C} 29$ & $177.34(17)$ \\
\hline $\mathrm{C} 16-\mathrm{C} 7-\mathrm{C} 8-\mathrm{C} 9$ & $0.4(3)$ & $\mathrm{C} 27-\mathrm{C} 28-\mathrm{C} 29-\mathrm{C} 30$ & $0.0(3)$ \\
\hline $\mathrm{C} 2-\mathrm{C} 7-\mathrm{C} 8-\mathrm{C} 9$ & $-177.0(2)$ & $\mathrm{C} 31-\mathrm{N} 2-\mathrm{C} 30-\mathrm{C} 25$ & $1.8(3)$ \\
\hline $\mathrm{C} 7-\mathrm{C} 8-\mathrm{C} 9-\mathrm{C} 10$ & $-0.4(4)$ & $\mathrm{C} 31-\mathrm{N} 2-\mathrm{C} 30-\mathrm{C} 29$ & $-179.23(19)$ \\
\hline $\mathrm{C} 8-\mathrm{C} 9-\mathrm{C} 10-\mathrm{C} 11$ & $0.0(4)$ & $\mathrm{C} 24-\mathrm{C} 25-\mathrm{C} 30-\mathrm{N} 2$ & $-4.3(3)$ \\
\hline $\mathrm{C} 9-\mathrm{C} 10-\mathrm{C} 11-\mathrm{C} 12$ & $179.7(3)$ & $\mathrm{C} 26-\mathrm{C} 25-\mathrm{C} 30-\mathrm{N} 2$ & $175.47(18)$ \\
\hline $\mathrm{C} 9-\mathrm{C} 10-\mathrm{C} 11-\mathrm{C} 16$ & $0.3(3)$ & $\mathrm{C} 24-\mathrm{C} 25-\mathrm{C} 30-\mathrm{C} 29$ & $176.72(18)$ \\
\hline $\mathrm{C} 10-\mathrm{C} 11-\mathrm{C} 12-\mathrm{C} 13$ & $-179.6(3)$ & $\mathrm{C} 26-\mathrm{C} 25-\mathrm{C} 30-\mathrm{C} 29$ & $-3.5(3)$ \\
\hline $\mathrm{C} 16-\mathrm{C} 11-\mathrm{C} 12-\mathrm{C} 13$ & $-0.2(4)$ & $\mathrm{C} 28-\mathrm{C} 29-\mathrm{C} 30-\mathrm{N} 2$ & $-176.2(2)$ \\
\hline $\mathrm{C} 11-\mathrm{C} 12-\mathrm{C} 13-\mathrm{C} 14$ & $0.3(5)$ & $\mathrm{C} 28-\mathrm{C} 29-\mathrm{C} 30-\mathrm{C} 25$ & $2.8(3)$ \\
\hline $\mathrm{C} 12-\mathrm{C} 13-\mathrm{C} 14-\mathrm{C} 15$ & $0.5(5)$ & $\mathrm{C} 30-\mathrm{N} 2-\mathrm{C} 31-\mathrm{O} 2$ & $-175.89(17)$ \\
\hline $\mathrm{C} 13-\mathrm{C} 14-\mathrm{C} 15-\mathrm{C} 16$ & $-1.4(4)$ & $\mathrm{C} 30-\mathrm{N} 2-\mathrm{C} 31-\mathrm{C} 23$ & $3.4(3)$ \\
\hline $\mathrm{C} 14-\mathrm{C} 15-\mathrm{C} 16-\mathrm{C} 11$ & $1.4(3)$ & $\mathrm{C} 32-\mathrm{O} 2-\mathrm{C} 31-\mathrm{N} 2$ & $3.0(3)$ \\
\hline $\mathrm{C} 14-\mathrm{C} 15-\mathrm{C} 16-\mathrm{C} 7$ & $-179.7(2)$ & $\mathrm{C} 32-\mathrm{O} 2-\mathrm{C} 31-\mathrm{C} 23$ & $-176.38(18)$ \\
\hline $\mathrm{C} 10-\mathrm{C} 11-\mathrm{C} 16-\mathrm{C} 15$ & $178.8(2)$ & $\mathrm{C} 24-\mathrm{C} 23-\mathrm{C} 31-\mathrm{N} 2$ & $-5.7(3)$ \\
\hline $\mathrm{C} 12-\mathrm{C} 11-\mathrm{C} 16-\mathrm{C} 15$ & $-0.6(3)$ & $\mathrm{C} 1-\mathrm{C} 23-\mathrm{C} 31-\mathrm{N} 2$ & $179.65(18)$ \\
\hline $\mathrm{C} 10-\mathrm{C} 11-\mathrm{C} 16-\mathrm{C} 7$ & $-0.2(3)$ & $\mathrm{C} 24-\mathrm{C} 23-\mathrm{C} 31-\mathrm{O} 2$ & $173.63(16)$ \\
\hline $\mathrm{C} 12-\mathrm{C} 11-\mathrm{C} 16-\mathrm{C} 7$ & $-179.6(2)$ & $\mathrm{C} 1-\mathrm{C} 23-\mathrm{C} 31-\mathrm{O} 2$ & $-1.1(2)$ \\
\hline $\mathrm{C} 8-\mathrm{C} 7-\mathrm{C} 16-\mathrm{C} 15$ & $-179.1(2)$ & $\mathrm{O} 3-\mathrm{C} 33-\mathrm{C} 34-\mathrm{C} 35$ & $-169.4(2)$ \\
\hline $\mathrm{C} 2-\mathrm{C} 7-\mathrm{C} 16-\mathrm{C} 15$ & $-1.9(3)$ & $\mathrm{O} 4-\mathrm{C} 33-\mathrm{C} 34-\mathrm{C} 35$ & $9.7(3)$ \\
\hline
\end{tabular}




\begin{tabular}{llll}
$\mathrm{C} 8-\mathrm{C} 7-\mathrm{C} 16-\mathrm{C} 11$ & $-0.2(3)$ & $\mathrm{O} 3-\mathrm{C} 33-\mathrm{C} 34-\mathrm{C} 39$ & $9.6(3)$ \\
$\mathrm{C} 2-\mathrm{C} 7-\mathrm{C} 16-\mathrm{C} 11$ & $177.02(17)$ & $\mathrm{O} 4-\mathrm{C} 33-\mathrm{C} 34-\mathrm{C} 39$ & $-171.3(2)$ \\
$\mathrm{C} 23-\mathrm{C} 1-\mathrm{C} 17-\mathrm{C} 22$ & $46.1(2)$ & $\mathrm{C} 39-\mathrm{C} 34-\mathrm{C} 35-\mathrm{C} 36$ & $-1.2(4)$ \\
$\mathrm{C} 2-\mathrm{C} 1-\mathrm{C} 17-\mathrm{C} 22$ & $-82.5(2)$ & $\mathrm{C} 33-\mathrm{C} 34-\mathrm{C} 35-\mathrm{C} 36$ & $177.8(3)$ \\
$\mathrm{C} 23-\mathrm{C} 1-\mathrm{C} 17-\mathrm{C} 18$ & $-134.71(18)$ & $\mathrm{C} 34-\mathrm{C} 35-\mathrm{C} 36-\mathrm{C} 37$ & $0.6(5)$ \\
$\mathrm{C} 2-\mathrm{C} 1-\mathrm{C} 17-\mathrm{C} 18$ & $96.7(2)$ & $\mathrm{C} 35-\mathrm{C} 36-\mathrm{C} 37-\mathrm{C} 38$ & $0.1(5)$ \\
$\mathrm{C} 22-\mathrm{C} 17-\mathrm{C} 18-\mathrm{C} 19$ & $-0.5(3)$ & $\mathrm{C} 36-\mathrm{C} 37-\mathrm{C} 38-\mathrm{C} 39$ & $-0.1(5)$ \\
$\mathrm{C} 1-\mathrm{C} 17-\mathrm{C} 18-\mathrm{C} 19$ & $-179.7(2)$ & $\mathrm{C} 37-\mathrm{C} 38-\mathrm{C} 39-\mathrm{C} 34$ & $-0.5(5)$ \\
$\mathrm{C} 17-\mathrm{C} 18-\mathrm{C} 19-\mathrm{C} 20$ & $-0.2(4)$ & $\mathrm{C} 35-\mathrm{C} 34-\mathrm{C} 39-\mathrm{C} 38$ & $1.1(4)$ \\
$\mathrm{C} 18-\mathrm{C} 19-\mathrm{C} 20-\mathrm{C} 21$ & $1.1(4)$ & $\mathrm{C} 33-\mathrm{C} 34-\mathrm{C} 39-\mathrm{C} 38$ & $-177.9(3)$ \\
\hline
\end{tabular}

Hydrogen-bond geometry $\left(A,{ }^{\circ}\right)$

\begin{tabular}{|c|c|c|c|c|}
\hline$D-\mathrm{H} \cdots A$ & $D-\mathrm{H}$ & $\mathrm{H} \cdots A$ & $D \cdots A$ & $D-\mathrm{H} \cdots A$ \\
\hline $\mathrm{O} 1-\mathrm{H} 1 B \cdots \mathrm{O} 5$ & 0.84 & 1.87 & $2.681(2)$ & 164 \\
\hline $\mathrm{O} 5-\mathrm{H} 5 D^{\cdots} \cdots 4^{\mathrm{i}}$ & $0.90(4)$ & $1.85(4)$ & $2.724(2)$ & $163(3)$ \\
\hline $\mathrm{O} 5-\mathrm{H} 5 E \cdots \mathrm{O} 3$ & $0.77(3)$ & $1.96(3)$ & $2.724(2)$ & $172(4)$ \\
\hline $\mathrm{O} 6-\mathrm{H} 6 E^{\cdots} \mathrm{N} 2$ & 0.87 & 2.38 & $3.148(13)$ & 147 \\
\hline $\mathrm{N} 1-\mathrm{H} 1 \cdots \mathrm{O} 4^{\mathrm{i}}$ & 1.00 & 1.64 & $2.643(2)$ & 178 \\
\hline $\mathrm{C} 5-\mathrm{H} 5 C^{\cdots} \cdot \mathrm{Br} 1^{\mathrm{ii}}$ & 0.98 & 3.09 & $3.910(2)$ & 142 \\
\hline $\mathrm{C} 6-\mathrm{H} 6 A \cdots \mathrm{O} 3$ & 0.98 & 2.53 & $3.465(3)$ & 161 \\
\hline $\mathrm{C} 6-\mathrm{H} 6 B^{\cdots} \cdots 6^{\mathrm{iii}}$ & 0.98 & 2.28 & 3.249 (13) & 169 \\
\hline $\mathrm{C} 28-\mathrm{H} 28 \cdots \mathrm{O} 5^{\mathrm{ii}}$ & 0.95 & 2.59 & $3.421(3)$ & 146 \\
\hline
\end{tabular}

Symmetry codes: (i) $-x+1, y-1 / 2,-z$; (ii) $-x+2, y-1 / 2,-z$; (iii) $x-1, y, z$.

[4-(6-Bromo-2-methoxyquinolin-3-yl)-3-hydroxy-3-(naphthalen-1-yl)-4-phenylbutyl]dimethylazanium benzoate acetonitrile 0.742 -solvate monohydrate $(4 \mathrm{~b})$

Crystal data

$\mathrm{C}_{32} \mathrm{H}_{32} \mathrm{BrN}_{2} \mathrm{O}_{2}^{+} \cdot \mathrm{C}_{7} \mathrm{H}_{5} \mathrm{O}_{2}^{-} \cdot 0.742 \mathrm{C}_{2} \mathrm{H}_{3} \mathrm{~N} \cdot \mathrm{H}_{2} \mathrm{O}$

$M_{r}=726.10$

Monoclinic, $P 2_{1}$

$a=12.8661(8) \AA$

$b=8.0386(5) \AA$

$c=17.4704(10) \AA$

$\beta=101.093(3)^{\circ}$

$V=1773.13(19) \AA^{3}$

$Z=2$

$F(000)=757$

$D_{\mathrm{x}}=1.360 \mathrm{Mg} \mathrm{m}^{-3}$

$\mathrm{Cu} K \alpha$ radiation, $\lambda=1.54178 \AA$

Cell parameters from 9023 reflections

$\theta=2.6-79.1^{\circ}$

$\mu=1.97 \mathrm{~mm}^{-1}$

$T=150 \mathrm{~K}$

Needle, colourless

$0.31 \times 0.05 \times 0.05 \mathrm{~mm}$

\section{Data collection}

Bruker D8 Quest

diffractometer with PhotonIII_C14 chargeintegrating and photon counting pixel array detector

Radiation source: I-mu-S microsource X-ray tube

Laterally graded multilayer (Goebel) mirror monochromator

Detector resolution: 7.4074 pixels $\mathrm{mm}^{-1}$ $\omega$ and phi scans
Absorption correction: multi-scan

(SADABS2016; Krause et al., 2015)

$T_{\min }=0.599, T_{\max }=0.754$

39739 measured reflections

7360 independent reflections

6750 reflections with $I>2 \sigma(I)$

$R_{\text {int }}=0.060$

$\theta_{\max }=80.1^{\circ}, \theta_{\min }=2.6^{\circ}$

$h=-16 \rightarrow 16$

$k=-8 \rightarrow 10$

$l=-22 \rightarrow 22$ 


\section{Refinement}

Refinement on $F^{2}$

Least-squares matrix: full

$R\left[F^{2}>2 \sigma\left(F^{2}\right)\right]=0.035$

$w R\left(F^{2}\right)=0.085$

$S=1.06$

7360 reflections

515 parameters

195 restraints

Primary atom site location: isomorphous structure methods

Secondary atom site location: difference Fourier map
Hydrogen site location: mixed

$\mathrm{H}$ atoms treated by a mixture of independent and constrained refinement

$w=1 /\left[\sigma^{2}\left(F_{0}^{2}\right)+(0.0233 P)^{2}+1.042 P\right]$

where $P=\left(F_{\mathrm{o}}{ }^{2}+2 F_{\mathrm{c}}{ }^{2}\right) / 3$

$(\Delta / \sigma)_{\max }=0.001$

$\Delta \rho_{\max }=0.40 \mathrm{e}^{-3}$

$\Delta \rho_{\min }=-0.50$ e $\AA^{-3}$

Absolute structure: Flack $x$ determined using 2778 quotients $[(\mathrm{I}+)-(\mathrm{I}-)] /[(\mathrm{I}+)+(\mathrm{I}-)]$ (Parsons et al., 2013)

Absolute structure parameter: $0.004(8)$

\section{Special details}

Geometry. All esds (except the esd in the dihedral angle between two 1.s. planes) are estimated using the full covariance matrix. The cell esds are taken into account individually in the estimation of esds in distances, angles and torsion angles; correlations between esds in cell parameters are only used when they are defined by crystal symmetry. An approximate (isotropic) treatment of cell esds is used for estimating esds involving l.s. planes.

Fractional atomic coordinates and isotropic or equivalent isotropic displacement parameters $\left(\AA^{2}\right)$

\begin{tabular}{llllll}
\hline & $x$ & $y$ & $z$ & $U_{\text {iso }} * / U_{\text {eq }}$ & Occ. $(<1)$ \\
\hline Br1 & $1.09186(3)$ & $0.23149(6)$ & $-0.10436(2)$ & $0.04184(12)$ & \\
O1 & $0.7542(2)$ & $0.3259(3)$ & $0.20992(15)$ & $0.0271(5)$ & \\
H1B & $0.734(4)$ & $0.292(2)$ & $0.168(3)$ & $0.041^{*}$ \\
O2 & $1.08646(19)$ & $0.0320(3)$ & $0.35039(14)$ & $0.0297(5)$ & \\
O3 & $0.4583(2)$ & $0.2868(4)$ & $0.05847(18)$ & $0.0497(8)$ & \\
O4 & $0.3385(2)$ & $0.4448(4)$ & $-0.01724(15)$ & $0.0373(6)$ \\
O5 & $0.6707(3)$ & $0.2738(4)$ & $0.05844(16)$ & $0.0387(7)$ \\
H5D & $0.674(5)$ & $0.174(4)$ & $0.041(3)$ & $0.058^{*}$ \\
H5E & $0.607(2)$ & $0.288(7)$ & $0.058(3)$ & $0.058^{*}$ \\
N1 & $0.5911(2)$ & $-0.1342(4)$ & $0.14353(17)$ & $0.0272(6)$ \\
H1 & 0.618783 & -0.106871 & 0.095314 & $0.033^{*}$ \\
N2 & $1.1414(2)$ & $0.0196(4)$ & $0.23270(18)$ & $0.0292(6)$ \\
C1 & $0.9000(2)$ & $0.1962(4)$ & $0.29767(17)$ & $0.0228(7)$ \\
H1A & 0.913127 & 0.113403 & 0.341185 & $0.027^{*}$ \\
C2 & $0.7781(3)$ & $0.1907(4)$ & $0.26263(18)$ & $0.0228(7)$ \\
C3 & $0.7471(3)$ & $0.0265(4)$ & $0.21840(19)$ & $0.0234(7)$ \\
H3A & 0.772164 & 0.028938 & 0.168253 & $0.028^{*}$ \\
H3B & 0.782695 & -0.067448 & 0.249584 & $0.028^{*}$ \\
C4 & $0.6277(3)$ & $-0.0017(5)$ & $0.20251(19)$ & $0.0249(7)$ \\
H4A & 0.605984 & -0.032128 & 0.252035 & $0.030^{*}$ \\
H4B & 0.591887 & 0.103914 & 0.184090 & $0.030^{*}$ \\
C5 & $0.6308(3)$ & $-0.3018(5)$ & $0.1703(2)$ & $0.0391(9)$ \\
H5A & 0.605300 & -0.383658 & 0.129406 & $0.059^{*}$ \\
H5B & 0.604938 & -0.331264 & 0.217728 & $0.059^{*}$ \\
H5C & 0.708438 & -0.301159 & 0.181360 & $0.059^{*}$ \\
C6 & $0.4732(3)$ & $-0.1344(6)$ & $0.1230(2)$ & $0.0366(9)$ \\
H6A & 0.448325 & -0.025168 & 0.102189 & $0.055^{*}$ &
\end{tabular}




\begin{tabular}{|c|c|c|c|c|}
\hline H6B & 0.444151 & -0.158068 & 0.169733 & $0.055^{*}$ \\
\hline $\mathrm{H} 6 \mathrm{C}$ & 0.449601 & -0.219985 & 0.083535 & $0.055^{*}$ \\
\hline C7 & $0.7184(2)$ & $0.2225(5)$ & $0.33036(16)$ & $0.0237(6)$ \\
\hline $\mathrm{C} 8$ & $0.6696(3)$ & $0.3737(5)$ & $0.3321(2)$ & $0.0322(8)$ \\
\hline H8 & 0.670541 & 0.448836 & 0.290297 & $0.039 *$ \\
\hline C9 & $0.6182(3)$ & $0.4236(6)$ & $0.3928(2)$ & $0.0391(9)$ \\
\hline H9 & 0.586471 & 0.530466 & 0.391630 & $0.047^{*}$ \\
\hline $\mathrm{C} 10$ & $0.6143(3)$ & $0.3178(6)$ & $0.4529(2)$ & $0.0371(9)$ \\
\hline H10 & 0.578544 & 0.350206 & 0.493248 & $0.045^{*}$ \\
\hline C11 & $0.6629(3)$ & $0.1604(5)$ & $0.4556(2)$ & $0.0328(8)$ \\
\hline $\mathrm{C} 12$ & $0.6601(4)$ & $0.0520(6)$ & $0.5190(2)$ & $0.0464(11)$ \\
\hline H12 & 0.624115 & 0.086173 & 0.559045 & $0.056^{*}$ \\
\hline C13 & $0.7070(5)$ & -0.0985 (7) & $0.5241(3)$ & $0.0578(14)$ \\
\hline H13 & 0.704267 & -0.168798 & 0.567337 & $0.069^{*}$ \\
\hline $\mathrm{C} 14$ & $0.7601(4)$ & $-0.1511(6)$ & $0.4650(3)$ & 0.0477 (11) \\
\hline H14 & 0.793019 & -0.257285 & 0.468543 & $0.057^{*}$ \\
\hline C15 & $0.7646(3)$ & $-0.0505(5)$ & $0.4024(2)$ & $0.0329(8)$ \\
\hline H15 & 0.800873 & -0.088643 & 0.363250 & $0.040^{*}$ \\
\hline $\mathrm{C} 16$ & 0.7167 (3) & $0.1088(5)$ & $0.3945(2)$ & $0.0261(7)$ \\
\hline $\mathrm{C} 17$ & 0.9317 (3) & 0.3644 (4) & $0.33358(19)$ & $0.0243(7)$ \\
\hline C18 & $0.9390(3)$ & $0.3895(5)$ & $0.41323(19)$ & $0.0287(7)$ \\
\hline H18 & 0.925588 & 0.299474 & 0.445188 & $0.034 *$ \\
\hline C19 & 0.9654 (3) & $0.5436(5)$ & $0.4466(2)$ & $0.0319(8)$ \\
\hline H19 & 0.970429 & 0.558180 & 0.501130 & $0.038^{*}$ \\
\hline $\mathrm{C} 20$ & $0.9846(3)$ & $0.6768(5)$ & 0.4009 (2) & $0.0340(8)$ \\
\hline $\mathrm{H} 20$ & 1.002007 & 0.782928 & 0.423684 & $0.041 *$ \\
\hline $\mathrm{C} 21$ & $0.9780(3)$ & $0.6530(5)$ & $0.3214(2)$ & $0.0341(8)$ \\
\hline $\mathrm{H} 21$ & 0.991414 & 0.743347 & 0.289646 & $0.041^{*}$ \\
\hline $\mathrm{C} 22$ & $0.9520(3)$ & $0.4987(5)$ & $0.2883(2)$ & $0.0301(8)$ \\
\hline $\mathrm{H} 22$ & 0.947839 & 0.484164 & 0.233828 & $0.036^{*}$ \\
\hline $\mathrm{C} 23$ & $0.9726(3)$ & 0.1487 (4) & $0.2416(2)$ & $0.0241(7)$ \\
\hline $\mathrm{C} 24$ & $0.9580(3)$ & 0.1938 (4) & $0.16468(18)$ & $0.0242(7)$ \\
\hline $\mathrm{H} 24$ & 0.896018 & 0.253246 & 0.141633 & $0.029^{*}$ \\
\hline $\mathrm{C} 25$ & 1.0347 (3) & $0.1528(5)$ & $0.1189(2)$ & $0.0263(7)$ \\
\hline $\mathrm{C} 26$ & $1.0240(3)$ & $0.2018(5)$ & $0.04000(19)$ & $0.0289(8)$ \\
\hline $\mathrm{H} 26$ & 0.964322 & 0.264196 & 0.015139 & $0.035^{*}$ \\
\hline $\mathrm{C} 27$ & $1.1014(3)$ & $0.1574(5)$ & $0.0004(2)$ & $0.0312(8)$ \\
\hline $\mathrm{C} 28$ & $1.1884(3)$ & $0.0584(5)$ & 0.0338 (2) & $0.0361(9)$ \\
\hline $\mathrm{H} 28$ & 1.238547 & 0.023743 & 0.003564 & $0.043^{*}$ \\
\hline $\mathrm{C} 29$ & $1.2000(3)$ & $0.0124(6)$ & 0.1104 (3) & $0.0378(9)$ \\
\hline H29 & 1.259208 & -0.052812 & 0.133734 & $0.045^{*}$ \\
\hline $\mathrm{C} 30$ & $1.1246(3)$ & $0.0612(5)$ & $0.1550(2)$ & $0.0285(7)$ \\
\hline $\mathrm{C} 31$ & $1.0703(3)$ & $0.0644(4)$ & $0.2727(2)$ & $0.0251(7)$ \\
\hline $\mathrm{C} 32$ & $1.1850(3)$ & $-0.0452(6)$ & $0.3846(3)$ & $0.0427(10)$ \\
\hline $\mathrm{H} 32 \mathrm{~A}$ & 1.191281 & -0.152129 & 0.358991 & $0.064 *$ \\
\hline H32B & 1.187118 & -0.063515 & 0.440374 & $0.064 *$ \\
\hline $\mathrm{H} 32 \mathrm{C}$ & 1.243771 & 0.027348 & 0.377926 & $0.064 *$ \\
\hline C33 & $0.3819(3)$ & $0.3840(5)$ & $0.0484(2)$ & $0.0310(8)$ \\
\hline
\end{tabular}




$\begin{array}{llllll}\text { C34 } & 0.3376(12) & 0.439(3) & 0.1162(6) & 0.030(2) & 0.742(7) \\ \text { C35 } & 0.2342(10) & 0.5018(15) & 0.1055(5) & 0.0338(19) & 0.742(7) \\ \text { H35 } & 0.193862 & 0.513732 & 0.054183 & 0.041^{*} & 0.742(7) \\ \text { C36 } & 0.1905(9) & 0.5461(17) & 0.1683(5) & 0.047(2) & 0.742(7) \\ \text { H36 } & 0.118077 & 0.577683 & 0.160811 & 0.057^{*} & 0.742(7) \\ \text { C37 } & 0.2530(7) & 0.5447(12) & 0.2437(5) & 0.0531(19) & 0.742(7) \\ \text { H37 } & 0.225227 & 0.586384 & 0.286502 & 0.064^{*} & 0.742(7) \\ \text { C38 } & 0.3550(6) & 0.4828(10) & 0.2555(4) & 0.0467(16) & 0.742(7) \\ \text { H38 } & 0.396204 & 0.475671 & 0.306703 & 0.056^{*} & 0.742(7) \\ \text { C39 } & 0.3966(5) & 0.4313(9) & 0.1920(4) & 0.0371(14) & 0.742(7) \\ \text { H39 } & 0.466935 & 0.389697 & 0.200206 & 0.044^{*} & 0.742(7) \\ \text { C34B } & 0.331(4) & 0.429(8) & 0.1208(16) & 0.0242(7) & 0.258(7) \\ \text { C35B } & 0.239(3) & 0.526(5) & 0.1165(17) & 0.035(4) & 0.258(7) \\ \text { H35B } & 0.202750 & 0.564683 & 0.067136 & 0.042^{*} & 0.258(7) \\ \text { C36B } & 0.202(3) & 0.567(5) & 0.1825(16) & 0.044(4) & 0.258(7) \\ \text { H36B } & 0.150076 & 0.651410 & 0.181779 & 0.053^{*} & 0.258(7) \\ \text { C37B } & 0.2432(18) & 0.479(3) & 0.2514(14) & 0.047(3) & 0.258(7) \\ \text { H37B } & 0.205535 & 0.477535 & 0.293157 & 0.056^{*} & 0.258(7) \\ \text { C38B } & 0.3375(16) & 0.396(3) & 0.2586(11) & 0.047(3) & 0.258(7) \\ \text { H38B } & 0.373369 & 0.359002 & 0.308434 & 0.057^{*} & 0.258(7) \\ \text { C39B } & 0.3805(17) & 0.365(3) & 0.1932(11) & 0.039(3) & 0.258(7) \\ \text { H39B } & 0.443068 & 0.299799 & 0.197365 & 0.047^{*} & 0.258(7) \\ \text { N3 } & 0.4791(5) & -0.2008(8) & 0.3556(4) & 0.0620(18) & 0.742(7) \\ \text { C40 } & 0.4502(5) & -0.0703(9) & 0.3379(4) & 0.0477(16) & 0.742(7) \\ \text { C41 } & 0.4178(6) & 0.0985(10) & 0.3164(5) & 0.063(2) & 0.742(7) \\ \text { H41A } & 0.473979 & 0.155009 & 0.295727 & 0.12(4)^{*} & 0.742(7) \\ \text { H41B } & 0.404449 & 0.158210 & 0.362451 & 0.14(5)^{*} & 0.742(7) \\ \text { H41C } & 0.352857 & 0.096518 & 0.276432 & 0.06(2)^{*} & 0.742(7)\end{array}$

Atomic displacement parameters $\left(\AA^{2}\right)$

\begin{tabular}{lllllll}
\hline & $U^{11}$ & $U^{22}$ & $U^{33}$ & $U^{12}$ & $U^{13}$ & $U^{23}$ \\
\hline Br1 & $0.0403(2)$ & $0.0597(3)$ & $0.02848(18)$ & $-0.0089(2)$ & $0.01393(14)$ & $-0.0069(2)$ \\
O1 & $0.0346(14)$ & $0.0229(13)$ & $0.0218(12)$ & $0.0020(10)$ & $0.0004(10)$ & $0.0026(9)$ \\
O2 & $0.0255(12)$ & $0.0329(14)$ & $0.0287(13)$ & $0.0057(10)$ & $-0.0002(10)$ & $0.0040(11)$ \\
O3 & $0.0501(17)$ & $0.053(2)$ & $0.0513(17)$ & $0.0218(14)$ & $0.0217(14)$ & $0.0106(14)$ \\
O4 & $0.0429(16)$ & $0.0428(17)$ & $0.0267(13)$ & $0.0012(13)$ & $0.0080(11)$ & $0.0042(12)$ \\
O5 & $0.0430(15)$ & $0.0396(19)$ & $0.0296(14)$ & $0.0001(12)$ & $-0.0030(12)$ & $0.0026(11)$ \\
N1 & $0.0259(15)$ & $0.0300(17)$ & $0.0247(14)$ & $-0.0036(12)$ & $0.0020(11)$ & $-0.0002(12)$ \\
N2 & $0.0236(14)$ & $0.0298(17)$ & $0.0348(16)$ & $0.0020(12)$ & $0.0075(12)$ & $-0.0010(13)$ \\
C1 & $0.0249(15)$ & $0.025(2)$ & $0.0189(14)$ & $0.0024(13)$ & $0.0057(11)$ & $0.0019(12)$ \\
C2 & $0.0264(15)$ & $0.0230(19)$ & $0.0186(14)$ & $0.0018(12)$ & $0.0036(12)$ & $0.0011(12)$ \\
C3 & $0.0253(16)$ & $0.0233(17)$ & $0.0216(15)$ & $0.0032(13)$ & $0.0043(12)$ & $-0.0013(13)$ \\
C4 & $0.0269(17)$ & $0.0279(19)$ & $0.0200(16)$ & $-0.0004(14)$ & $0.0049(13)$ & $-0.0038(13)$ \\
C5 & $0.042(2)$ & $0.030(2)$ & $0.044(2)$ & $-0.0019(17)$ & $0.0062(16)$ & $0.0004(17)$ \\
C6 & $0.0284(19)$ & $0.046(2)$ & $0.0318(19)$ & $-0.0067(16)$ & $-0.0032(15)$ & $0.0013(17)$ \\
C7 & $0.0213(13)$ & $0.0262(17)$ & $0.0226(13)$ & $0.0029(16)$ & $0.0014(10)$ & $-0.0024(16)$ \\
C8 & $0.0338(19)$ & $0.034(2)$ & $0.0292(18)$ & $0.0083(16)$ & $0.0055(15)$ & $0.0040(15)$
\end{tabular}




\begin{tabular}{|c|c|c|c|c|c|c|}
\hline C9 & $0.042(2)$ & $0.037(2)$ & $0.038(2)$ & $0.0124(18)$ & $0.0085(17)$ & $-0.0050(17)$ \\
\hline $\mathrm{C} 10$ & $0.036(2)$ & $0.046(2)$ & $0.032(2)$ & $0.0070(18)$ & $0.0116(17)$ & $-0.0042(17)$ \\
\hline $\mathrm{C} 11$ & $0.0315(19)$ & $0.042(2)$ & $0.0249(18)$ & $0.0011(16)$ & $0.0068(15)$ & $0.0003(15)$ \\
\hline $\mathrm{C} 12$ & $0.056(3)$ & $0.054(3)$ & $0.035(2)$ & $0.006(2)$ & $0.025(2)$ & $0.007(2)$ \\
\hline $\mathrm{C} 13$ & $0.077(3)$ & $0.062(3)$ & $0.043(3)$ & 0.015 & $0.033(2)$ & $0.023(2)$ \\
\hline $\mathrm{C} 14$ & $0.064(3)$ & $0.043(3)$ & $0.041(2)$ & $0.011(2)$ & $0.023(2)$ & $0.0151(19)$ \\
\hline $\mathrm{C} 15$ & $0.037(2)$ & $0.031(2)$ & $0.0324(19)$ & $0.0024(16)$ & $0.0111(16)$ & $0.0040(15)$ \\
\hline $\mathrm{C} 16$ & $0.0240(16)$ & 0.0307 (19) & $0.0239(17)$ & $0.0004(13)$ & $0.0052(13)$ & $0.0014(13)$ \\
\hline $\mathrm{C} 17$ & $0.0229(16)$ & $0.0269(18)$ & $0.0223(16)$ & $0.0006(13)$ & $0.0022(12)$ & $0.0014(13)$ \\
\hline $\mathrm{C} 18$ & $0.0379(19)$ & $0.0275(19)$ & $0.0197(16)$ & $-0.0008(15)$ & $0.0027(14)$ & $0.0023(13)$ \\
\hline C19 & $0.044(2)$ & $0.031(2)$ & $0.0197(16)$ & $-0.0037(16)$ & $0.0048(15)$ & $-0.0011(14)$ \\
\hline $\mathrm{C} 20$ & $0.048(2)$ & $0.027(2)$ & $0.0261(17)$ & $-0.0024(15)$ & $0.0028(15)$ & $-0.0029(13)$ \\
\hline $\mathrm{C} 21$ & $0.049(2)$ & $0.029(2)$ & $0.0240(17)$ & $-0.0065(17)$ & $0.0074(15)$ & $0.0036(15)$ \\
\hline $\mathrm{C} 22$ & $0.041(2)$ & $0.030(2)$ & $0.0204(16)$ & $-0.0047(15)$ & $0.0078(14)$ & $-0.0004(14)$ \\
\hline $\mathrm{C} 23$ & $0.0220(16)$ & $0.0228(17)$ & $0.0275(17)$ & $0.0007(13)$ & $0.0045(13)$ & $-0.0019(13)$ \\
\hline C24 & $0.0239(14)$ & $0.0259(19)$ & $0.0232(14)$ & $0.0014(12)$ & $0.0060(12)$ & $-0.0018(12)$ \\
\hline $\mathrm{C} 25$ & $0.0244(16)$ & $0.0262(18)$ & $0.0300(18)$ & $-0.0026(13)$ & $0.0091(13)$ & $-0.0038(14)$ \\
\hline $\mathrm{C} 26$ & $0.0284(16)$ & $0.034(2)$ & $0.0251(15)$ & $-0.0016(14)$ & $0.0087(12)$ & $-0.0039(14)$ \\
\hline $\mathrm{C} 27$ & $0.0326(19)$ & $0.034(2)$ & $0.0287(18)$ & $-0.0096(16)$ & $0.0104(15)$ & $-0.0061(15)$ \\
\hline C28 & 0.0308 (19) & $0.039(2)$ & $0.043(2)$ & $-0.0012(16)$ & $0.0185(16)$ & $-0.0075(17)$ \\
\hline C29 & $0.0283(19)$ & $0.038(2)$ & $0.049(2)$ & $0.0038(16)$ & $0.0120(17)$ & $-0.0006(18)$ \\
\hline $\mathrm{C} 30$ & $0.0250(17)$ & $0.0272(19)$ & $0.0341(19)$ & $-0.0004(14)$ & $0.0074(14)$ & $-0.0022(15)$ \\
\hline $\mathrm{C} 31$ & $0.0253(16)$ & $0.0220(17)$ & $0.0270(17)$ & $-0.0010(13)$ & $0.0025(13)$ & $0.0024(13)$ \\
\hline $\mathrm{C} 32$ & $0.030(2)$ & $0.051(3)$ & $0.042(2)$ & $0.0104(18)$ & $-0.0054(17)$ & 0.0067 (19) \\
\hline $\mathrm{C} 33$ & $0.0353(19)$ & $0.029(2)$ & $0.0299(18)$ & $-0.0005(15)$ & $0.0096(15)$ & $0.0002(15)$ \\
\hline $\mathrm{C} 34$ & $0.030(3)$ & $0.035(4)$ & $0.027(3)$ & $-0.001(3)$ & $0.006(2)$ & $-0.001(2)$ \\
\hline $\mathrm{C} 35$ & $0.034(3)$ & $0.035(4)$ & $0.032(3)$ & 0.004 (3) & $0.006(3)$ & $-0.005(3)$ \\
\hline $\mathrm{C} 36$ & $0.041(4)$ & $0.060(5)$ & $0.042(4)$ & $0.012(4)$ & $0.010(3)$ & -0.013 \\
\hline $\mathrm{C} 37$ & $0.065(4)$ & $0.058(5)$ & $0.040(3)$ & $0.010(4)$ & $0.019(3)$ & -0.009 \\
\hline $\mathrm{C} 38$ & $0.056(3)$ & $0.053(4)$ & $0.030(3)$ & $0.002(3)$ & $0.005(2)$ & $-0.007(3)$ \\
\hline C39 & $0.036(3)$ & $0.040(4)$ & $0.034(3)$ & $0.000(3)$ & $0.003(2)$ & $-0.001(3)$ \\
\hline C34B & $0.0239(14)$ & $0.0259(19)$ & $0.0232(14)$ & $0.0014(12)$ & $0.0060(12)$ & $-0.0018(12)$ \\
\hline C35B & $0.031(6)$ & $0.042(7)$ & $0.030(6)$ & $0.003(6)$ & $0.003(5)$ & $-0.006(6)$ \\
\hline C36B & $0.044(6)$ & $0.052(7)$ & $0.037(6)$ & $0.009(6)$ & $0.012(6)$ & $-0.017(6)$ \\
\hline C37B & $0.053(6)$ & $0.055(7)$ & $0.036(6)$ & $0.003(6)$ & $0.018(5)$ & $-0.009(6)$ \\
\hline C38B & $0.052(6)$ & $0.056(7)$ & $0.036(5)$ & $0.004(6)$ & $0.012(5)$ & $-0.003(6)$ \\
\hline C39B & $0.040(6)$ & $0.044(6)$ & $0.033(5)$ & $0.004(6)$ & $0.008(5)$ & $-0.004(6)$ \\
\hline N3 & $0.077(4)$ & $0.038(3)$ & $0.078(4)$ & $0.002(3)$ & $0.030(3)$ & $0.015(3)$ \\
\hline $\mathrm{C} 40$ & $0.045(3)$ & $0.053(4)$ & $0.051(4)$ & -0.008 & $0.024(3)$ & $-0.002(3)$ \\
\hline C41 & 0.049 (4) & $0.055(4)$ & $0.092(6)$ & 0.009 (3) & $0.032(4)$ & 0.007 (4) \\
\hline
\end{tabular}

Geometric parameters $\left(\AA,{ }^{o}\right)$

\begin{tabular}{llll}
\hline $\mathrm{Br} 1-\mathrm{C} 27$ & $1.905(4)$ & $\mathrm{C} 18-\mathrm{H} 18$ & 0.9500 \\
$\mathrm{O} 1-\mathrm{C} 2$ & $1.419(4)$ & $\mathrm{C} 19-\mathrm{C} 20$ & $1.386(5)$ \\
$\mathrm{O} 1-\mathrm{H} 1 \mathrm{~B}$ & $0.77(5)$ & $\mathrm{C} 19-\mathrm{H} 19$ & 0.9500 \\
$\mathrm{O} 2-\mathrm{C} 31$ & $1.358(4)$ & $\mathrm{C} 20-\mathrm{C} 21$ & $1.387(5)$ \\
$\mathrm{O} 2-\mathrm{C} 32$ & $1.434(4)$ & $\mathrm{C} 20-\mathrm{H} 20$ & 0.9500
\end{tabular}




\begin{tabular}{|c|c|c|c|}
\hline $\mathrm{O} 3-\mathrm{C} 33$ & $1.241(5)$ & $\mathrm{C} 21-\mathrm{C} 22$ & $1.383(5)$ \\
\hline $\mathrm{O} 4-\mathrm{C} 33$ & $1.273(5)$ & $\mathrm{C} 21-\mathrm{H} 21$ & 0.9500 \\
\hline $\mathrm{O} 5-\mathrm{H} 5 \mathrm{D}$ & $0.86(3)$ & $\mathrm{C} 22-\mathrm{H} 22$ & 0.9500 \\
\hline O5-H5E & $0.82(3)$ & $\mathrm{C} 23-\mathrm{C} 24$ & $1.370(5)$ \\
\hline $\mathrm{N} 1-\mathrm{C} 5$ & $1.484(5)$ & $\mathrm{C} 23-\mathrm{C} 31$ & $1.440(5)$ \\
\hline $\mathrm{N} 1-\mathrm{C} 6$ & $1.490(5)$ & $\mathrm{C} 24-\mathrm{C} 25$ & $1.423(5)$ \\
\hline $\mathrm{N} 1-\mathrm{C} 4$ & $1.494(4)$ & $\mathrm{C} 24-\mathrm{H} 24$ & 0.9500 \\
\hline $\mathrm{N} 1-\mathrm{H} 1$ & 1.0000 & $\mathrm{C} 25-\mathrm{C} 30$ & $1.413(5)$ \\
\hline $\mathrm{N} 2-\mathrm{C} 31$ & $1.305(5)$ & $\mathrm{C} 25-\mathrm{C} 26$ & $1.415(5)$ \\
\hline $\mathrm{N} 2-\mathrm{C} 30$ & $1.374(5)$ & $\mathrm{C} 26-\mathrm{C} 27$ & $1.365(5)$ \\
\hline $\mathrm{C} 1-\mathrm{C} 17$ & $1.513(5)$ & $\mathrm{C} 26-\mathrm{H} 26$ & 0.9500 \\
\hline $\mathrm{C} 1-\mathrm{C} 23$ & $1.526(4)$ & $\mathrm{C} 27-\mathrm{C} 28$ & $1.406(6)$ \\
\hline $\mathrm{C} 1-\mathrm{C} 2$ & $1.572(4)$ & $\mathrm{C} 28-\mathrm{C} 29$ & $1.369(6)$ \\
\hline $\mathrm{C} 1-\mathrm{H} 1 \mathrm{~A}$ & 1.0000 & $\mathrm{C} 28-\mathrm{H} 28$ & 0.9500 \\
\hline $\mathrm{C} 2-\mathrm{C} 3$ & $1.543(5)$ & $\mathrm{C} 29-\mathrm{C} 30$ & $1.412(5)$ \\
\hline $\mathrm{C} 2-\mathrm{C} 7$ & $1.551(4)$ & C29-H29 & 0.9500 \\
\hline $\mathrm{C} 3-\mathrm{C} 4$ & $1.525(5)$ & $\mathrm{C} 32-\mathrm{H} 32 \mathrm{~A}$ & 0.9800 \\
\hline $\mathrm{C} 3-\mathrm{H} 3 \mathrm{~A}$ & 0.9900 & $\mathrm{C} 32-\mathrm{H} 32 \mathrm{~B}$ & 0.9800 \\
\hline $\mathrm{C} 3-\mathrm{H} 3 \mathrm{~B}$ & 0.9900 & $\mathrm{C} 32-\mathrm{H} 32 \mathrm{C}$ & 0.9800 \\
\hline $\mathrm{C} 4-\mathrm{H} 4 \mathrm{~A}$ & 0.9900 & C33-C34 & $1.478(12)$ \\
\hline $\mathrm{C} 4-\mathrm{H} 4 \mathrm{~B}$ & 0.9900 & $\mathrm{C} 33-\mathrm{C} 34 \mathrm{~B}$ & $1.57(3)$ \\
\hline $\mathrm{C} 5-\mathrm{H} 5 \mathrm{~A}$ & 0.9800 & C34-C39 & $1.395(11)$ \\
\hline C5-H5B & 0.9800 & $\mathrm{C} 34-\mathrm{C} 35$ & $1.401(10)$ \\
\hline $\mathrm{C} 5-\mathrm{H} 5 \mathrm{C}$ & 0.9800 & $\mathrm{C} 35-\mathrm{C} 36$ & $1.373(9)$ \\
\hline C6-H6A & 0.9800 & $\mathrm{C} 35-\mathrm{H} 35$ & 0.9500 \\
\hline C6- $\mathrm{H} 6 \mathrm{~B}$ & 0.9800 & $\mathrm{C} 36-\mathrm{C} 37$ & $1.405(10)$ \\
\hline C6- $\mathrm{H} 6 \mathrm{C}$ & 0.9800 & $\mathrm{C} 36-\mathrm{H} 36$ & 0.9500 \\
\hline $\mathrm{C} 7-\mathrm{C} 8$ & $1.370(6)$ & C $37-$ C 38 & $1.381(10)$ \\
\hline $\mathrm{C} 7-\mathrm{C} 16$ & $1.449(5)$ & $\mathrm{C} 37-\mathrm{H} 37$ & 0.9500 \\
\hline $\mathrm{C} 8-\mathrm{C} 9$ & $1.411(5)$ & C38-C39 & $1.385(8)$ \\
\hline $\mathrm{C} 8-\mathrm{H} 8$ & 0.9500 & C38-H38 & 0.9500 \\
\hline $\mathrm{C} 9-\mathrm{C} 10$ & $1.359(6)$ & C39-H39 & 0.9500 \\
\hline C9-H9 & 0.9500 & $\mathrm{C} 34 \mathrm{~B}-\mathrm{C} 39 \mathrm{~B}$ & $1.40(2)$ \\
\hline $\mathrm{C} 10-\mathrm{C} 11$ & $1.408(6)$ & $\mathrm{C} 34 \mathrm{~B}-\mathrm{C} 35 \mathrm{~B}$ & $1.41(2)$ \\
\hline $\mathrm{C} 10-\mathrm{H} 10$ & 0.9500 & $\mathrm{C} 35 \mathrm{~B}-\mathrm{C} 36 \mathrm{~B}$ & $1.37(2)$ \\
\hline $\mathrm{C} 11-\mathrm{C} 12$ & $1.416(6)$ & $\mathrm{C} 35 \mathrm{~B}-\mathrm{H} 35 \mathrm{~B}$ & 0.9500 \\
\hline $\mathrm{C} 11-\mathrm{C} 16$ & $1.440(5)$ & $\mathrm{C} 36 \mathrm{~B}-\mathrm{C} 37 \mathrm{~B}$ & $1.41(2)$ \\
\hline $\mathrm{C} 12-\mathrm{C} 13$ & $1.347(7)$ & $\mathrm{C} 36 \mathrm{~B}-\mathrm{H} 36 \mathrm{~B}$ & 0.9500 \\
\hline $\mathrm{C} 12-\mathrm{H} 12$ & 0.9500 & $\mathrm{C} 37 \mathrm{~B}-\mathrm{C} 38 \mathrm{~B}$ & $1.37(2)$ \\
\hline $\mathrm{C} 13-\mathrm{C} 14$ & $1.408(6)$ & $\mathrm{C} 37 \mathrm{~B}-\mathrm{H} 37 \mathrm{~B}$ & 0.9500 \\
\hline C13-H13 & 0.9500 & $\mathrm{C} 38 \mathrm{~B}-\mathrm{C} 39 \mathrm{~B}$ & 1.384 (19) \\
\hline $\mathrm{C} 14-\mathrm{C} 15$ & $1.370(5)$ & $\mathrm{C} 38 \mathrm{~B}-\mathrm{H} 38 \mathrm{~B}$ & 0.9500 \\
\hline C14-H14 & 0.9500 & С39B-H39B & 0.9500 \\
\hline $\mathrm{C} 15-\mathrm{C} 16$ & $1.417(5)$ & N3-C40 & $1.136(9)$ \\
\hline $\mathrm{C} 15-\mathrm{H} 15$ & 0.9500 & $\mathrm{C} 40-\mathrm{C} 41$ & $1.448(10)$ \\
\hline $\mathrm{C} 17-\mathrm{C} 18$ & $1.391(5)$ & $\mathrm{C} 41-\mathrm{H} 41 \mathrm{~A}$ & 0.9800 \\
\hline $\mathrm{C} 17-\mathrm{C} 22$ & $1.393(5)$ & $\mathrm{C} 41-\mathrm{H} 41 \mathrm{~B}$ & 0.9800 \\
\hline $\mathrm{C} 18-\mathrm{C} 19$ & $1.383(5)$ & $\mathrm{C} 41-\mathrm{H} 41 \mathrm{C}$ & 0.9800 \\
\hline
\end{tabular}




\begin{tabular}{|c|c|c|c|}
\hline $\mathrm{C} 2-\mathrm{O} 1-\mathrm{H} 1 \mathrm{~B}$ & 109.5 & $\mathrm{C} 22-\mathrm{C} 21-\mathrm{C} 20$ & $120.4(3)$ \\
\hline $\mathrm{C} 31-\mathrm{O} 2-\mathrm{C} 32$ & $116.9(3)$ & $\mathrm{C} 22-\mathrm{C} 21-\mathrm{H} 21$ & 119.8 \\
\hline $\mathrm{H} 5 \mathrm{D}-\mathrm{O} 5-\mathrm{H} 5 \mathrm{E}$ & $104(5)$ & $\mathrm{C} 20-\mathrm{C} 21-\mathrm{H} 21$ & 119.8 \\
\hline $\mathrm{C} 5-\mathrm{N} 1-\mathrm{C} 6$ & $110.4(3)$ & $\mathrm{C} 21-\mathrm{C} 22-\mathrm{C} 17$ & $121.0(3)$ \\
\hline $\mathrm{C} 5-\mathrm{N} 1-\mathrm{C} 4$ & $112.7(3)$ & $\mathrm{C} 21-\mathrm{C} 22-\mathrm{H} 22$ & 119.5 \\
\hline $\mathrm{C} 6-\mathrm{N} 1-\mathrm{C} 4$ & $109.8(3)$ & $\mathrm{C} 17-\mathrm{C} 22-\mathrm{H} 22$ & 119.5 \\
\hline $\mathrm{C} 5-\mathrm{N} 1-\mathrm{H} 1$ & 107.9 & $\mathrm{C} 24-\mathrm{C} 23-\mathrm{C} 31$ & $116.1(3)$ \\
\hline $\mathrm{C} 6-\mathrm{N} 1-\mathrm{H} 1$ & 107.9 & $\mathrm{C} 24-\mathrm{C} 23-\mathrm{C} 1$ & $125.3(3)$ \\
\hline $\mathrm{C} 4-\mathrm{N} 1-\mathrm{H} 1$ & 107.9 & $\mathrm{C} 31-\mathrm{C} 23-\mathrm{C} 1$ & $118.2(3)$ \\
\hline $\mathrm{C} 31-\mathrm{N} 2-\mathrm{C} 30$ & $117.9(3)$ & $\mathrm{C} 23-\mathrm{C} 24-\mathrm{C} 25$ & $120.7(3)$ \\
\hline $\mathrm{C} 17-\mathrm{C} 1-\mathrm{C} 23$ & $109.9(3)$ & $\mathrm{C} 23-\mathrm{C} 24-\mathrm{H} 24$ & 119.6 \\
\hline $\mathrm{C} 17-\mathrm{C} 1-\mathrm{C} 2$ & $110.9(3)$ & $\mathrm{C} 25-\mathrm{C} 24-\mathrm{H} 24$ & 119.6 \\
\hline $\mathrm{C} 23-\mathrm{C} 1-\mathrm{C} 2$ & $115.5(3)$ & $\mathrm{C} 30-\mathrm{C} 25-\mathrm{C} 26$ & $119.9(3)$ \\
\hline $\mathrm{C} 17-\mathrm{C} 1-\mathrm{H} 1 \mathrm{~A}$ & 106.7 & $\mathrm{C} 30-\mathrm{C} 25-\mathrm{C} 24$ & $117.8(3)$ \\
\hline $\mathrm{C} 23-\mathrm{C} 1-\mathrm{H} 1 \mathrm{~A}$ & 106.7 & $\mathrm{C} 26-\mathrm{C} 25-\mathrm{C} 24$ & $122.4(3)$ \\
\hline $\mathrm{C} 2-\mathrm{C} 1-\mathrm{H} 1 \mathrm{~A}$ & 106.7 & $\mathrm{C} 27-\mathrm{C} 26-\mathrm{C} 25$ & $118.6(3)$ \\
\hline $\mathrm{O} 1-\mathrm{C} 2-\mathrm{C} 3$ & $109.1(3)$ & $\mathrm{C} 27-\mathrm{C} 26-\mathrm{H} 26$ & 120.7 \\
\hline $\mathrm{O} 1-\mathrm{C} 2-\mathrm{C} 7$ & $107.0(3)$ & $\mathrm{C} 25-\mathrm{C} 26-\mathrm{H} 26$ & 120.7 \\
\hline $\mathrm{C} 3-\mathrm{C} 2-\mathrm{C} 7$ & $113.9(3)$ & $\mathrm{C} 26-\mathrm{C} 27-\mathrm{C} 28$ & $122.5(4)$ \\
\hline $\mathrm{O} 1-\mathrm{C} 2-\mathrm{C} 1$ & $107.8(3)$ & $\mathrm{C} 26-\mathrm{C} 27-\mathrm{Br} 1$ & $119.2(3)$ \\
\hline $\mathrm{C} 3-\mathrm{C} 2-\mathrm{C} 1$ & $111.2(3)$ & $\mathrm{C} 28-\mathrm{C} 27-\mathrm{Br} 1$ & $118.3(3)$ \\
\hline $\mathrm{C} 7-\mathrm{C} 2-\mathrm{C} 1$ & $107.6(2)$ & $\mathrm{C} 29-\mathrm{C} 28-\mathrm{C} 27$ & $119.1(3)$ \\
\hline $\mathrm{C} 4-\mathrm{C} 3-\mathrm{C} 2$ & $111.8(3)$ & $\mathrm{C} 29-\mathrm{C} 28-\mathrm{H} 28$ & 120.4 \\
\hline $\mathrm{C} 4-\mathrm{C} 3-\mathrm{H} 3 \mathrm{~A}$ & 109.2 & $\mathrm{C} 27-\mathrm{C} 28-\mathrm{H} 28$ & 120.4 \\
\hline $\mathrm{C} 2-\mathrm{C} 3-\mathrm{H} 3 \mathrm{~A}$ & 109.2 & $\mathrm{C} 28-\mathrm{C} 29-\mathrm{C} 30$ & $120.6(4)$ \\
\hline $\mathrm{C} 4-\mathrm{C} 3-\mathrm{H} 3 \mathrm{~B}$ & 109.2 & $\mathrm{C} 28-\mathrm{C} 29-\mathrm{H} 29$ & 119.7 \\
\hline $\mathrm{C} 2-\mathrm{C} 3-\mathrm{H} 3 \mathrm{~B}$ & 109.2 & $\mathrm{C} 30-\mathrm{C} 29-\mathrm{H} 29$ & 119.7 \\
\hline $\mathrm{H} 3 \mathrm{~A}-\mathrm{C} 3-\mathrm{H} 3 \mathrm{~B}$ & 107.9 & $\mathrm{~N} 2-\mathrm{C} 30-\mathrm{C} 29$ & $118.9(3)$ \\
\hline $\mathrm{N} 1-\mathrm{C} 4-\mathrm{C} 3$ & $113.9(3)$ & $\mathrm{N} 2-\mathrm{C} 30-\mathrm{C} 25$ & $121.9(3)$ \\
\hline $\mathrm{N} 1-\mathrm{C} 4-\mathrm{H} 4 \mathrm{~A}$ & 108.8 & $\mathrm{C} 29-\mathrm{C} 30-\mathrm{C} 25$ & $119.2(3)$ \\
\hline $\mathrm{C} 3-\mathrm{C} 4-\mathrm{H} 4 \mathrm{~A}$ & 108.8 & $\mathrm{~N} 2-\mathrm{C} 31-\mathrm{O} 2$ & $119.6(3)$ \\
\hline $\mathrm{N} 1-\mathrm{C} 4-\mathrm{H} 4 \mathrm{~B}$ & 108.8 & $\mathrm{~N} 2-\mathrm{C} 31-\mathrm{C} 23$ & $125.4(3)$ \\
\hline $\mathrm{C} 3-\mathrm{C} 4-\mathrm{H} 4 \mathrm{~B}$ & 108.8 & $\mathrm{O} 2-\mathrm{C} 31-\mathrm{C} 23$ & $115.0(3)$ \\
\hline $\mathrm{H} 4 \mathrm{~A}-\mathrm{C} 4-\mathrm{H} 4 \mathrm{~B}$ & 107.7 & $\mathrm{O} 2-\mathrm{C} 32-\mathrm{H} 32 \mathrm{~A}$ & 109.5 \\
\hline $\mathrm{N} 1-\mathrm{C} 5-\mathrm{H} 5 \mathrm{~A}$ & 109.5 & $\mathrm{O} 2-\mathrm{C} 32-\mathrm{H} 32 \mathrm{~B}$ & 109.5 \\
\hline $\mathrm{N} 1-\mathrm{C} 5-\mathrm{H} 5 \mathrm{~B}$ & 109.5 & $\mathrm{H} 32 \mathrm{~A}-\mathrm{C} 32-\mathrm{H} 32 \mathrm{~B}$ & 109.5 \\
\hline $\mathrm{H} 5 \mathrm{~A}-\mathrm{C} 5-\mathrm{H} 5 \mathrm{~B}$ & 109.5 & $\mathrm{O} 2-\mathrm{C} 32-\mathrm{H} 32 \mathrm{C}$ & 109.5 \\
\hline $\mathrm{N} 1-\mathrm{C} 5-\mathrm{H} 5 \mathrm{C}$ & 109.5 & $\mathrm{H} 32 \mathrm{~A}-\mathrm{C} 32-\mathrm{H} 32 \mathrm{C}$ & 109.5 \\
\hline $\mathrm{H} 5 \mathrm{~A}-\mathrm{C} 5-\mathrm{H} 5 \mathrm{C}$ & 109.5 & $\mathrm{H} 32 \mathrm{~B}-\mathrm{C} 32-\mathrm{H} 32 \mathrm{C}$ & 109.5 \\
\hline $\mathrm{H} 5 \mathrm{~B}-\mathrm{C} 5-\mathrm{H} 5 \mathrm{C}$ & 109.5 & $\mathrm{O} 3-\mathrm{C} 33-\mathrm{O} 4$ & $124.6(4)$ \\
\hline $\mathrm{N} 1-\mathrm{C} 6-\mathrm{H} 6 \mathrm{~A}$ & 109.5 & $\mathrm{O} 3-\mathrm{C} 33-\mathrm{C} 34$ & $119.5(5)$ \\
\hline $\mathrm{N} 1-\mathrm{C} 6-\mathrm{H} 6 \mathrm{~B}$ & 109.5 & $\mathrm{O} 4-\mathrm{C} 33-\mathrm{C} 34$ & $115.9(5)$ \\
\hline $\mathrm{H} 6 \mathrm{~A}-\mathrm{C} 6-\mathrm{H} 6 \mathrm{~B}$ & 109.5 & $\mathrm{O} 3-\mathrm{C} 33-\mathrm{C} 34 \mathrm{~B}$ & $118.1(11)$ \\
\hline $\mathrm{N} 1-\mathrm{C} 6-\mathrm{H} 6 \mathrm{C}$ & 109.5 & $\mathrm{O} 4-\mathrm{C} 33-\mathrm{C} 34 \mathrm{~B}$ & $117.2(11)$ \\
\hline $\mathrm{H} 6 \mathrm{~A}-\mathrm{C} 6-\mathrm{H} 6 \mathrm{C}$ & 109.5 & $\mathrm{C} 39-\mathrm{C} 34-\mathrm{C} 35$ & $118.2(9)$ \\
\hline $\mathrm{H} 6 \mathrm{~B}-\mathrm{C} 6-\mathrm{H} 6 \mathrm{C}$ & 109.5 & $\mathrm{C} 39-\mathrm{C} 34-\mathrm{C} 33$ & $121.6(9)$ \\
\hline $\mathrm{C} 8-\mathrm{C} 7-\mathrm{C} 16$ & $117.8(3)$ & $\mathrm{C} 35-\mathrm{C} 34-\mathrm{C} 33$ & $120.2(9)$ \\
\hline
\end{tabular}




\begin{tabular}{|c|c|c|c|}
\hline $\mathrm{C} 8-\mathrm{C} 7-\mathrm{C} 2$ & $117.3(3)$ & $\mathrm{C} 36-\mathrm{C} 35-\mathrm{C} 34$ & $120.7(8)$ \\
\hline $\mathrm{C} 16-\mathrm{C} 7-\mathrm{C} 2$ & $124.7(3)$ & $\mathrm{C} 36-\mathrm{C} 35-\mathrm{H} 35$ & 119.6 \\
\hline $\mathrm{C} 7-\mathrm{C} 8-\mathrm{C} 9$ & $123.5(4)$ & $\mathrm{C} 34-\mathrm{C} 35-\mathrm{H} 35$ & 119.6 \\
\hline $\mathrm{C} 7-\mathrm{C} 8-\mathrm{H} 8$ & 118.3 & $\mathrm{C} 35-\mathrm{C} 36-\mathrm{C} 37$ & $119.9(8)$ \\
\hline $\mathrm{C} 9-\mathrm{C} 8-\mathrm{H} 8$ & 118.3 & $\mathrm{C} 35-\mathrm{C} 36-\mathrm{H} 36$ & 120.1 \\
\hline $\mathrm{C} 10-\mathrm{C} 9-\mathrm{C} 8$ & $119.6(4)$ & $\mathrm{C} 37-\mathrm{C} 36-\mathrm{H} 36$ & 120.1 \\
\hline $\mathrm{C} 10-\mathrm{C} 9-\mathrm{H} 9$ & 120.2 & $\mathrm{C} 38-\mathrm{C} 37-\mathrm{C} 36$ & $119.9(7)$ \\
\hline $\mathrm{C} 8-\mathrm{C} 9-\mathrm{H} 9$ & 120.2 & $\mathrm{C} 38-\mathrm{C} 37-\mathrm{H} 37$ & 120.0 \\
\hline $\mathrm{C} 9-\mathrm{C} 10-\mathrm{C} 11$ & $120.4(4)$ & $\mathrm{C} 36-\mathrm{C} 37-\mathrm{H} 37$ & 120.0 \\
\hline $\mathrm{C} 9-\mathrm{C} 10-\mathrm{H} 10$ & 119.8 & $\mathrm{C} 37-\mathrm{C} 38-\mathrm{C} 39$ & $119.4(6)$ \\
\hline $\mathrm{C} 11-\mathrm{C} 10-\mathrm{H} 10$ & 119.8 & $\mathrm{C} 37-\mathrm{C} 38-\mathrm{H} 38$ & 120.3 \\
\hline $\mathrm{C} 10-\mathrm{C} 11-\mathrm{C} 12$ & $120.1(4)$ & $\mathrm{C} 39-\mathrm{C} 38-\mathrm{H} 38$ & 120.3 \\
\hline $\mathrm{C} 10-\mathrm{C} 11-\mathrm{C} 16$ & $120.5(4)$ & $\mathrm{C} 38-\mathrm{C} 39-\mathrm{C} 34$ & $121.6(7)$ \\
\hline $\mathrm{C} 12-\mathrm{C} 11-\mathrm{C} 16$ & $119.5(4)$ & C38-C39-H39 & 119.2 \\
\hline $\mathrm{C} 13-\mathrm{C} 12-\mathrm{C} 11$ & $121.7(4)$ & $\mathrm{C} 34-\mathrm{C} 39-\mathrm{H} 39$ & 119.2 \\
\hline $\mathrm{C} 13-\mathrm{C} 12-\mathrm{H} 12$ & 119.2 & $\mathrm{C} 39 \mathrm{~B}-\mathrm{C} 34 \mathrm{~B}-\mathrm{C} 35 \mathrm{~B}$ & $119(2)$ \\
\hline $\mathrm{C} 11-\mathrm{C} 12-\mathrm{H} 12$ & 119.2 & $\mathrm{C} 39 \mathrm{~B}-\mathrm{C} 34 \mathrm{~B}-\mathrm{C} 33$ & $117(2)$ \\
\hline $\mathrm{C} 12-\mathrm{C} 13-\mathrm{C} 14$ & $119.8(4)$ & $\mathrm{C} 35 \mathrm{~B}-\mathrm{C} 34 \mathrm{~B}-\mathrm{C} 33$ & $124(2)$ \\
\hline $\mathrm{C} 12-\mathrm{C} 13-\mathrm{H} 13$ & 120.1 & $\mathrm{C} 36 \mathrm{~B}-\mathrm{C} 35 \mathrm{~B}-\mathrm{C} 34 \mathrm{~B}$ & $121(2)$ \\
\hline $\mathrm{C} 14-\mathrm{C} 13-\mathrm{H} 13$ & 120.1 & $\mathrm{C} 36 \mathrm{~B}-\mathrm{C} 35 \mathrm{~B}-\mathrm{H} 35 \mathrm{~B}$ & 119.6 \\
\hline $\mathrm{C} 15-\mathrm{C} 14-\mathrm{C} 13$ & $120.6(4)$ & $\mathrm{C} 34 \mathrm{~B}-\mathrm{C} 35 \mathrm{~B}-\mathrm{H} 35 \mathrm{~B}$ & 119.6 \\
\hline $\mathrm{C} 15-\mathrm{C} 14-\mathrm{H} 14$ & 119.7 & $\mathrm{C} 35 \mathrm{~B}-\mathrm{C} 36 \mathrm{~B}-\mathrm{C} 37 \mathrm{~B}$ & $118(2)$ \\
\hline $\mathrm{C} 13-\mathrm{C} 14-\mathrm{H} 14$ & 119.7 & $\mathrm{C} 35 \mathrm{~B}-\mathrm{C} 36 \mathrm{~B}-\mathrm{H} 36 \mathrm{~B}$ & 121.2 \\
\hline $\mathrm{C} 14-\mathrm{C} 15-\mathrm{C} 16$ & $121.8(4)$ & $\mathrm{C} 37 \mathrm{~B}-\mathrm{C} 36 \mathrm{~B}-\mathrm{H} 36 \mathrm{~B}$ & 121.2 \\
\hline $\mathrm{C} 14-\mathrm{C} 15-\mathrm{H} 15$ & 119.1 & $\mathrm{C} 38 \mathrm{~B}-\mathrm{C} 37 \mathrm{~B}-\mathrm{C} 36 \mathrm{~B}$ & $120(2)$ \\
\hline $\mathrm{C} 16-\mathrm{C} 15-\mathrm{H} 15$ & 119.1 & $\mathrm{C} 38 \mathrm{~B}-\mathrm{C} 37 \mathrm{~B}-\mathrm{H} 37 \mathrm{~B}$ & 119.8 \\
\hline $\mathrm{C} 15-\mathrm{C} 16-\mathrm{C} 11$ & $116.7(3)$ & $\mathrm{C} 36 \mathrm{~B}-\mathrm{C} 37 \mathrm{~B}-\mathrm{H} 37 \mathrm{~B}$ & 119.8 \\
\hline $\mathrm{C} 15-\mathrm{C} 16-\mathrm{C} 7$ & $125.1(3)$ & $\mathrm{C} 37 \mathrm{~B}-\mathrm{C} 38 \mathrm{~B}-\mathrm{C} 39 \mathrm{~B}$ & $120.0(17)$ \\
\hline $\mathrm{C} 11-\mathrm{C} 16-\mathrm{C} 7$ & $118.2(3)$ & $\mathrm{C} 37 \mathrm{~B}-\mathrm{C} 38 \mathrm{~B}-\mathrm{H} 38 \mathrm{~B}$ & 120.0 \\
\hline $\mathrm{C} 18-\mathrm{C} 17-\mathrm{C} 22$ & $118.1(3)$ & $\mathrm{C} 39 \mathrm{~B}-\mathrm{C} 38 \mathrm{~B}-\mathrm{H} 38 \mathrm{~B}$ & 120.0 \\
\hline $\mathrm{C} 18-\mathrm{C} 17-\mathrm{C} 1$ & $120.3(3)$ & $\mathrm{C} 38 \mathrm{~B}-\mathrm{C} 39 \mathrm{~B}-\mathrm{C} 34 \mathrm{~B}$ & $119.7(19)$ \\
\hline $\mathrm{C} 22-\mathrm{C} 17-\mathrm{C} 1$ & $121.5(3)$ & $\mathrm{C} 38 \mathrm{~B}-\mathrm{C} 39 \mathrm{~B}-\mathrm{H} 39 \mathrm{~B}$ & 120.2 \\
\hline $\mathrm{C} 19-\mathrm{C} 18-\mathrm{C} 17$ & $121.0(3)$ & $\mathrm{C} 34 \mathrm{~B}-\mathrm{C} 39 \mathrm{~B}-\mathrm{H} 39 \mathrm{~B}$ & 120.2 \\
\hline $\mathrm{C} 19-\mathrm{C} 18-\mathrm{H} 18$ & 119.5 & $\mathrm{~N} 3-\mathrm{C} 40-\mathrm{C} 41$ & $177.7(8)$ \\
\hline $\mathrm{C} 17-\mathrm{C} 18-\mathrm{H} 18$ & 119.5 & $\mathrm{C} 40-\mathrm{C} 41-\mathrm{H} 41 \mathrm{~A}$ & 109.5 \\
\hline $\mathrm{C} 18-\mathrm{C} 19-\mathrm{C} 20$ & $120.4(3)$ & $\mathrm{C} 40-\mathrm{C} 41-\mathrm{H} 41 \mathrm{~B}$ & 109.5 \\
\hline $\mathrm{C} 18-\mathrm{C} 19-\mathrm{H} 19$ & 119.8 & $\mathrm{H} 41 \mathrm{~A}-\mathrm{C} 41-\mathrm{H} 41 \mathrm{~B}$ & 109.5 \\
\hline $\mathrm{C} 20-\mathrm{C} 19-\mathrm{H} 19$ & 119.8 & $\mathrm{C} 40-\mathrm{C} 41-\mathrm{H} 41 \mathrm{C}$ & 109.5 \\
\hline $\mathrm{C} 19-\mathrm{C} 20-\mathrm{C} 21$ & $119.1(4)$ & $\mathrm{H} 41 \mathrm{~A}-\mathrm{C} 41-\mathrm{H} 41 \mathrm{C}$ & 109.5 \\
\hline $\mathrm{C} 19-\mathrm{C} 20-\mathrm{H} 20$ & 120.4 & $\mathrm{H} 41 \mathrm{~B}-\mathrm{C} 41-\mathrm{H} 41 \mathrm{C}$ & 109.5 \\
\hline $\mathrm{C} 21-\mathrm{C} 20-\mathrm{H} 20$ & 120.4 & & \\
\hline $\mathrm{C} 17-\mathrm{C} 1-\mathrm{C} 2-\mathrm{O} 1$ & $54.5(3)$ & $\mathrm{C} 17-\mathrm{C} 1-\mathrm{C} 23-\mathrm{C} 31$ & $87.0(4)$ \\
\hline $\mathrm{C} 23-\mathrm{C} 1-\mathrm{C} 2-\mathrm{O} 1$ & $-71.4(3)$ & $\mathrm{C} 2-\mathrm{C} 1-\mathrm{C} 23-\mathrm{C} 31$ & $-146.7(3)$ \\
\hline $\mathrm{C} 17-\mathrm{C} 1-\mathrm{C} 2-\mathrm{C} 3$ & $174.1(3)$ & $\mathrm{C} 31-\mathrm{C} 23-\mathrm{C} 24-\mathrm{C} 25$ & $2.2(5)$ \\
\hline $\mathrm{C} 23-\mathrm{C} 1-\mathrm{C} 2-\mathrm{C} 3$ & $48.2(4)$ & $\mathrm{C} 1-\mathrm{C} 23-\mathrm{C} 24-\mathrm{C} 25$ & $175.4(3)$ \\
\hline $\mathrm{C} 17-\mathrm{C} 1-\mathrm{C} 2-\mathrm{C} 7$ & $-60.6(3)$ & $\mathrm{C} 23-\mathrm{C} 24-\mathrm{C} 25-\mathrm{C} 30$ & $1.3(5)$ \\
\hline $\mathrm{C} 23-\mathrm{C} 1-\mathrm{C} 2-\mathrm{C} 7$ & $173.6(3)$ & $\mathrm{C} 23-\mathrm{C} 24-\mathrm{C} 25-\mathrm{C} 26$ & $-178.0(3)$ \\
\hline
\end{tabular}




\begin{tabular}{|c|c|}
\hline $\mathrm{O} 1-\mathrm{C} 2-\mathrm{C} 3-\mathrm{C} 4$ & $-76.1(3)$ \\
\hline $\mathrm{C} 7-\mathrm{C} 2-\mathrm{C} 3-\mathrm{C} 4$ & $43.4(4)$ \\
\hline $\mathrm{C} 1-\mathrm{C} 2-\mathrm{C} 3-\mathrm{C} 4$ & $165.1(3)$ \\
\hline $\mathrm{C} 5-\mathrm{N} 1-\mathrm{C} 4-\mathrm{C} 3$ & $65.3(4)$ \\
\hline $\mathrm{C} 6-\mathrm{N} 1-\mathrm{C} 4-\mathrm{C} 3$ & $-171.3(3)$ \\
\hline $\mathrm{C} 2-\mathrm{C} 3-\mathrm{C} 4-\mathrm{N} 1$ & $165.5(3)$ \\
\hline $\mathrm{O} 1-\mathrm{C} 2-\mathrm{C} 7-\mathrm{C} 8$ & $-8.1(4)$ \\
\hline $\mathrm{C} 3-\mathrm{C} 2-\mathrm{C} 7-\mathrm{C} 8$ & $-128.8(3)$ \\
\hline $\mathrm{C} 1-\mathrm{C} 2-\mathrm{C} 7-\mathrm{C} 8$ & $107.5(3)$ \\
\hline $\mathrm{O} 1-\mathrm{C} 2-\mathrm{C} 7-\mathrm{C} 16$ & $176.3(3)$ \\
\hline $\mathrm{C} 3-\mathrm{C} 2-\mathrm{C} 7-\mathrm{C} 16$ & $55.6(4)$ \\
\hline $\mathrm{C} 1-\mathrm{C} 2-\mathrm{C} 7-\mathrm{C} 16$ & $-68.1(4)$ \\
\hline $\mathrm{C} 16-\mathrm{C} 7-\mathrm{C} 8-\mathrm{C} 9$ & $-0.1(6)$ \\
\hline $\mathrm{C} 2-\mathrm{C} 7-\mathrm{C} 8-\mathrm{C} 9$ & $-175.9(3)$ \\
\hline $\mathrm{C} 7-\mathrm{C} 8-\mathrm{C} 9-\mathrm{C} 10$ & $-0.9(6)$ \\
\hline $\mathrm{C} 8-\mathrm{C} 9-\mathrm{C} 10-\mathrm{C} 11$ & $1.2(6)$ \\
\hline $\mathrm{C} 9-\mathrm{C} 10-\mathrm{C} 11-\mathrm{C} 12$ & $178.9(4)$ \\
\hline $\mathrm{C} 9-\mathrm{C} 10-\mathrm{C} 11-\mathrm{C} 16$ & $-0.5(6)$ \\
\hline $\mathrm{C} 10-\mathrm{C} 11-\mathrm{C} 12-\mathrm{C} 13$ & $-179.1(5)$ \\
\hline $\mathrm{C} 16-\mathrm{C} 11-\mathrm{C} 12-\mathrm{C} 13$ & $0.4(7)$ \\
\hline $\mathrm{C} 11-\mathrm{C} 12-\mathrm{C} 13-\mathrm{C} 14$ & $-0.3(8)$ \\
\hline $\mathrm{C} 12-\mathrm{C} 13-\mathrm{C} 14-\mathrm{C} 15$ & $0.1(8)$ \\
\hline $\mathrm{C} 13-\mathrm{C} 14-\mathrm{C} 15-\mathrm{C} 16$ & $0.0(7)$ \\
\hline $\mathrm{C} 14-\mathrm{C} 15-\mathrm{C} 16-\mathrm{C} 11$ & $0.1(6)$ \\
\hline $\mathrm{C} 14-\mathrm{C} 15-\mathrm{C} 16-\mathrm{C} 7$ & $179.7(4)$ \\
\hline $\mathrm{C} 10-\mathrm{C} 11-\mathrm{C} 16-\mathrm{C} 15$ & $179.2(4)$ \\
\hline $\mathrm{C} 12-\mathrm{C} 11-\mathrm{C} 16-\mathrm{C} 15$ & $-0.3(6)$ \\
\hline $\mathrm{C} 10-\mathrm{C} 11-\mathrm{C} 16-\mathrm{C} 7$ & $-0.5(5)$ \\
\hline $\mathrm{C} 12-\mathrm{C} 11-\mathrm{C} 16-\mathrm{C} 7$ & $-179.9(4)$ \\
\hline $\mathrm{C} 8-\mathrm{C} 7-\mathrm{C} 16-\mathrm{C} 15$ & $-178.9(4)$ \\
\hline $\mathrm{C} 2-\mathrm{C} 7-\mathrm{C} 16-\mathrm{C} 15$ & $-3.3(5)$ \\
\hline $\mathrm{C} 8-\mathrm{C} 7-\mathrm{C} 16-\mathrm{C} 11$ & $0.7(5)$ \\
\hline $\mathrm{C} 2-\mathrm{C} 7-\mathrm{C} 16-\mathrm{C} 11$ & $176.3(3)$ \\
\hline $\mathrm{C} 23-\mathrm{C} 1-\mathrm{C} 17-\mathrm{C} 18$ & $-135.1(3)$ \\
\hline $\mathrm{C} 2-\mathrm{C} 1-\mathrm{C} 17-\mathrm{C} 18$ & $95.9(4)$ \\
\hline $\mathrm{C} 23-\mathrm{C} 1-\mathrm{C} 17-\mathrm{C} 22$ & $46.4(4)$ \\
\hline $\mathrm{C} 2-\mathrm{C} 1-\mathrm{C} 17-\mathrm{C} 22$ & $-82.5(4)$ \\
\hline $\mathrm{C} 22-\mathrm{C} 17-\mathrm{C} 18-\mathrm{C} 19$ & $0.1(5)$ \\
\hline $\mathrm{C} 1-\mathrm{C} 17-\mathrm{C} 18-\mathrm{C} 19$ & $-178.4(3)$ \\
\hline $\mathrm{C} 17-\mathrm{C} 18-\mathrm{C} 19-\mathrm{C} 20$ & $0.4(6)$ \\
\hline $\mathrm{C} 18-\mathrm{C} 19-\mathrm{C} 20-\mathrm{C} 21$ & $-0.7(6)$ \\
\hline $\mathrm{C} 19-\mathrm{C} 20-\mathrm{C} 21-\mathrm{C} 22$ & $0.4(6)$ \\
\hline $\mathrm{C} 20-\mathrm{C} 21-\mathrm{C} 22-\mathrm{C} 17$ & $0.1(6)$ \\
\hline $\mathrm{C} 18-\mathrm{C} 17-\mathrm{C} 22-\mathrm{C} 21$ & $-0.3(6)$ \\
\hline $\mathrm{C} 1-\mathrm{C} 17-\mathrm{C} 22-\mathrm{C} 21$ & $178.2(3)$ \\
\hline $\mathrm{C} 17-\mathrm{C} 1-\mathrm{C} 23-\mathrm{C} 24$ & $-86.1(4)$ \\
\hline $\mathrm{C} 2-\mathrm{C} 1-\mathrm{C} 23-\mathrm{C} 24$ & $40.2(5)$ \\
\hline
\end{tabular}

\begin{tabular}{|c|c|}
\hline $\mathrm{C} 30-\mathrm{C} 25-\mathrm{C} 26-\mathrm{C} 27$ & $0.6(5)$ \\
\hline $\mathrm{C} 24-\mathrm{C} 25-\mathrm{C} 26-\mathrm{C} 27$ & $179.9(3)$ \\
\hline $\mathrm{C} 25-\mathrm{C} 26-\mathrm{C} 27-\mathrm{C} 28$ & $3.2(6)$ \\
\hline $\mathrm{C} 25-\mathrm{C} 26-\mathrm{C} 27-\mathrm{Br} 1$ & $-177.0(3)$ \\
\hline $\mathrm{C} 26-\mathrm{C} 27-\mathrm{C} 28-\mathrm{C} 29$ & $-4.1(6)$ \\
\hline $\mathrm{Br} 1-\mathrm{C} 27-\mathrm{C} 28-\mathrm{C} 29$ & $176.1(3)$ \\
\hline $\mathrm{C} 27-\mathrm{C} 28-\mathrm{C} 29-\mathrm{C} 30$ & $1.1(6)$ \\
\hline $\mathrm{C} 31-\mathrm{N} 2-\mathrm{C} 30-\mathrm{C} 29$ & $-179.3(4)$ \\
\hline $\mathrm{C} 31-\mathrm{N} 2-\mathrm{C} 30-\mathrm{C} 25$ & $1.4(5)$ \\
\hline $\mathrm{C} 28-\mathrm{C} 29-\mathrm{C} 30-\mathrm{N} 2$ & $-176.9(4)$ \\
\hline $\mathrm{C} 28-\mathrm{C} 29-\mathrm{C} 30-\mathrm{C} 25$ & $2.4(6)$ \\
\hline $\mathrm{C} 26-\mathrm{C} 25-\mathrm{C} 30-\mathrm{N} 2$ & $176.0(3)$ \\
\hline $\mathrm{C} 24-\mathrm{C} 25-\mathrm{C} 30-\mathrm{N} 2$ & $-3.3(5)$ \\
\hline $\mathrm{C} 26-\mathrm{C} 25-\mathrm{C} 30-\mathrm{C} 29$ & $-3.3(6)$ \\
\hline $\mathrm{C} 24-\mathrm{C} 25-\mathrm{C} 30-\mathrm{C} 29$ & $177.4(3)$ \\
\hline $\mathrm{C} 30-\mathrm{N} 2-\mathrm{C} 31-\mathrm{O} 2$ & $-177.1(3)$ \\
\hline $\mathrm{C} 30-\mathrm{N} 2-\mathrm{C} 31-\mathrm{C} 23$ & $2.6(5)$ \\
\hline $\mathrm{C} 32-\mathrm{O} 2-\mathrm{C} 31-\mathrm{N} 2$ & $2.4(5)$ \\
\hline $\mathrm{C} 32-\mathrm{O} 2-\mathrm{C} 31-\mathrm{C} 23$ & $-177.3(3)$ \\
\hline $\mathrm{C} 24-\mathrm{C} 23-\mathrm{C} 31-\mathrm{N} 2$ & $-4.4(5)$ \\
\hline $\mathrm{C} 1-\mathrm{C} 23-\mathrm{C} 31-\mathrm{N} 2$ & $-178.2(3)$ \\
\hline $\mathrm{C} 24-\mathrm{C} 23-\mathrm{C} 31-\mathrm{O} 2$ & $175.3(3)$ \\
\hline $\mathrm{C} 1-\mathrm{C} 23-\mathrm{C} 31-\mathrm{O} 2$ & $1.5(5)$ \\
\hline $\mathrm{O} 3-\mathrm{C} 33-\mathrm{C} 34-\mathrm{C} 39$ & $21(2)$ \\
\hline $\mathrm{O} 4-\mathrm{C} 33-\mathrm{C} 34-\mathrm{C} 39$ & $-157.5(13)$ \\
\hline $\mathrm{O} 3-\mathrm{C} 33-\mathrm{C} 34-\mathrm{C} 35$ & $-159.2(14)$ \\
\hline $\mathrm{O} 4-\mathrm{C} 33-\mathrm{C} 34-\mathrm{C} 35$ & $22(2)$ \\
\hline $\mathrm{C} 39-\mathrm{C} 34-\mathrm{C} 35-\mathrm{C} 36$ & $-3(3)$ \\
\hline $\mathrm{C} 33-\mathrm{C} 34-\mathrm{C} 35-\mathrm{C} 36$ & $177.0(13)$ \\
\hline $\mathrm{C} 34-\mathrm{C} 35-\mathrm{C} 36-\mathrm{C} 37$ & $6(2)$ \\
\hline $\mathrm{C} 35-\mathrm{C} 36-\mathrm{C} 37-\mathrm{C} 38$ & $-6.6(17)$ \\
\hline $\mathrm{C} 36-\mathrm{C} 37-\mathrm{C} 38-\mathrm{C} 39$ & $3.6(14)$ \\
\hline $\mathrm{C} 37-\mathrm{C} 38-\mathrm{C} 39-\mathrm{C} 34$ & $-0.6(16)$ \\
\hline $\mathrm{C} 35-\mathrm{C} 34-\mathrm{C} 39-\mathrm{C} 38$ & $0(2)$ \\
\hline $\mathrm{C} 33-\mathrm{C} 34-\mathrm{C} 39-\mathrm{C} 38$ & $180.0(12)$ \\
\hline $\mathrm{O} 3-\mathrm{C} 33-\mathrm{C} 34 \mathrm{~B}-\mathrm{C} 39 \mathrm{~B}$ & $4(6)$ \\
\hline $\mathrm{O} 4-\mathrm{C} 33-\mathrm{C} 34 \mathrm{~B}-\mathrm{C} 39 \mathrm{~B}$ & $-179(3)$ \\
\hline $\mathrm{O} 3-\mathrm{C} 33-\mathrm{C} 34 \mathrm{~B}-\mathrm{C} 35 \mathrm{~B}$ & $-175(5)$ \\
\hline $\mathrm{O} 4-\mathrm{C} 33-\mathrm{C} 34 \mathrm{~B}-\mathrm{C} 35 \mathrm{~B}$ & $2(7)$ \\
\hline $\mathrm{C} 39 \mathrm{~B}-\mathrm{C} 34 \mathrm{~B}-\mathrm{C} 35 \mathrm{~B}-\mathrm{C} 36 \mathrm{~B}$ & $4(8)$ \\
\hline $\mathrm{C} 33-\mathrm{C} 34 \mathrm{~B}-\mathrm{C} 35 \mathrm{~B}-\mathrm{C} 36 \mathrm{~B}$ & $-177(4)$ \\
\hline $\mathrm{C} 34 \mathrm{~B}-\mathrm{C} 35 \mathrm{~B}-\mathrm{C} 36 \mathrm{~B}-\mathrm{C} 37 \mathrm{~B}$ & $-14(6)$ \\
\hline $\mathrm{C} 35 \mathrm{~B}-\mathrm{C} 36 \mathrm{~B}-\mathrm{C} 37 \mathrm{~B}-\mathrm{C} 38 \mathrm{~B}$ & $19(5)$ \\
\hline $\mathrm{C} 36 \mathrm{~B}-\mathrm{C} 37 \mathrm{~B}-\mathrm{C} 38 \mathrm{~B}-\mathrm{C} 39 \mathrm{~B}$ & $-15(4)$ \\
\hline $\mathrm{C} 37 \mathrm{~B}-\mathrm{C} 38 \mathrm{~B}-\mathrm{C} 39 \mathrm{~B}-\mathrm{C} 34 \mathrm{~B}$ & $5(5)$ \\
\hline $\mathrm{C} 35 \mathrm{~B}-\mathrm{C} 34 \mathrm{~B}-\mathrm{C} 39 \mathrm{~B}-\mathrm{C} 38 \mathrm{~B}$ & $1(8)$ \\
\hline $\mathrm{C} 33-\mathrm{C} 34 \mathrm{~B}-\mathrm{C} 39 \mathrm{~B}-\mathrm{C} 38 \mathrm{~B}$ & $-178(3)$ \\
\hline
\end{tabular}


supporting information

Hydrogen-bond geometry $\left(A,{ }^{\circ}\right)$

\begin{tabular}{lllll}
\hline$D-\mathrm{H} \cdots A$ & $D-\mathrm{H}$ & $\mathrm{H} \cdots A$ & $D \cdots A$ & $D-\mathrm{H} \cdots A$ \\
\hline $\mathrm{O} 1-\mathrm{H} 1 B^{\cdots} \cdots \mathrm{O} 5$ & 0.77 & 1.94 & $2.691(4)$ & 163 \\
$\mathrm{O} 5-\mathrm{H} 5 D \cdots \mathrm{O} 4^{\mathrm{i}}$ & $0.86(3)$ & $1.89(3)$ & $2.738(4)$ & $168(6)$ \\
$\mathrm{O} 5-\mathrm{H} 5 E^{\cdots} \mathrm{O} 3$ & $0.82(3)$ & $1.92(3)$ & $2.734(4)$ & $172(6)$ \\
$\mathrm{N} 1-\mathrm{H} 1 \cdots \mathrm{O} 44^{\mathrm{i}}$ & 1.00 & 1.62 & $2.619(4)$ & 178 \\
$\mathrm{C} 5-\mathrm{H} 5 C \cdots \mathrm{Br} 1^{\mathrm{ii}}$ & 0.98 & 3.13 & $3.964(4)$ & 144 \\
$\mathrm{C} 6-\mathrm{H} 6 A \cdots \mathrm{O} 3$ & 0.98 & 2.63 & $3.562(6)$ & 159 \\
$\mathrm{C} 28-\mathrm{H} 28 \cdots \mathrm{O} 5^{\mathrm{ii}}$ & 0.95 & 2.66 & $3.498(5)$ & 148
\end{tabular}

Symmetry codes: (i) $-x+1, y-1 / 2,-z$; (ii) $-x+2, y-1 / 2,-z$. 